\title{
Ensemble of MRR and NDCG models for Visual Dialog
}

\author{
Idan Schwartz \\ Technion \\ NetApp \\ idanscecs.technion.ac.il
}

\begin{abstract}
Assessing an AI agent that can converse in human language and understand visual content is challenging. Generation metrics, such as BLEU scores favor correct syntax over semantics. Hence a discriminative approach is often used, where an agent ranks a set of candidate options. The mean reciprocal rank (MRR) metric evaluates the model performance by taking into account the rank of a single humanderived answer. This approach, however, raises a new challenge: the ambiguity and synonymy of answers, for instance, semantic equivalence (e.g., 'yeah' and 'yes'). To address this, the normalized discounted cumulative gain (NDCG) metric has been used to capture the relevance of all the correct answers via dense annotations. However, the NDCG metric favors the usually applicable uncertain answers such as 'I don't know.' Crafting a model that excels on both MRR and NDCG metrics is challenging (Murahari et al., 2020). Ideally, an AI agent should answer a human-like reply and validate the correctness of any answer. To address this issue, we describe a twostep non-parametric ranking approach that can merge strong MRR and NDCG models. Using our approach, we manage to keep most MRR state-of-the-art performance $(70.41 \% \mathrm{vs}$. $71.24 \%)$ and the NDCG state-of-the-art performance $(72.16 \%$ vs. $75.35 \%)$. Moreover, our approach won the recent Visual Dialog 2020 challenge. Source code is available at https: //github.com/idansc/mrr-ndcg.
\end{abstract}

\section{Introduction}

Das et al. (2017) introduced the task of Visual Dialog, which requires an agent to converse about visual input. Evaluating visually aware conversation should examine both linguistic properties and visual reasoning. Analysis of generative metrics for dialog often shows no correlation with human judgments (Liu et al., 2016). Hence, to evaluate the correctness of the candidate answers, a retrieval approach is preferred. Two metrics are standard,
Question: what is the nightstand made of ?

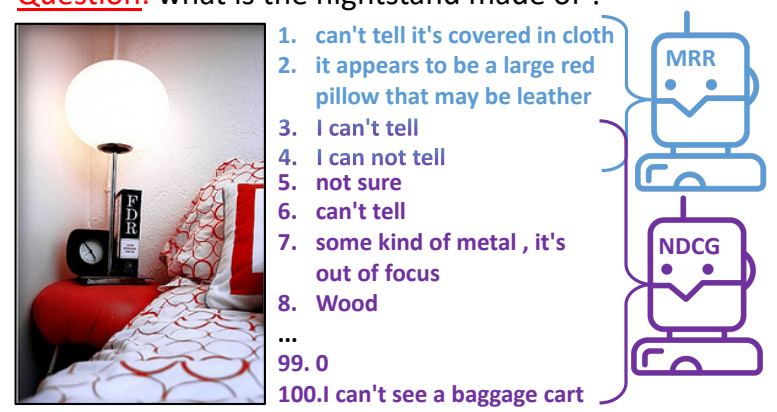

Figure 1: A visual dialog interaction. The question asks, "what is the nightstand made of ?". We show our final ranking, created by the ensemble of an MRR/NDCG models' rankings. The MRR/NDCG models are trained to optimize the MRR/NDCG metric. The MRR metric measures the number of retrievals to retrieve the human-derived answer. Hence, the MRR model favors human-like and detailed answers. On the other hand, the NDCG metric measures the rank of all the correct candidates based on dense annotation, which are often general and uncertain. Our ensemble approach seeks a minimal candidate set that is likely to contain the human-derived answer. The remaining candidates are ranked according to the NDCG model.

MRR and NDCG. The MRR metric focuses on a single human-derived ground-truth answer. Despite preferring the more human-like answer, the metric ignores many correct candidate answers. Differently, the NDCG considers the rank of all the correct answers. The metric relies on dense annotation, where three annotators were asked to mark all the correct candidate answers. However, the candidate answers are generated plausible answers. The analysis shows that the NDCG metric favors uncertain, generally correct answers, such as "not sure" (Murahari et al., 2020; Qi et al., 2020).

Prior work in visual dialog focused on a single metric. Ideally, an AI agent should answer humanlike and detailed reply (the MRR metric) and be able to validate the correctness of any answer (the NDCG metric). However, crafting a model that excels in both metrics is challenging (Murahari et al., 2020). To this end, we propose principals to ensemble the rankings of strong MRR and NDCG models. Our approach is to find a minimal set that is likely 
to hold the human-derived answer. This permits ranking the rest of the candidates according to the NDCG model. Our approach won the recent Visual Dialog 2020 challenge and achieved strong performance on both the MRR and the NDCG metrics simultaneously.

\section{Related Work}

Visual conversation evaluation: Early attempts to marry conversation with vision used street scene images, and binary questions (Geman et al., 2015). While binary answers are easy to verify, such an approach is limiting for an AI agent. On the other hand, analysis of generative metrics for dialog often show no correlation with human judgements (Liu et al., 2016). Intuitively, metrics like BLEU-scores rely on corresponding words with the ground-truth answer and often miss synonyms or the subjective nature. More importantly, generative metrics are geared toward textual assessment rather than visual reasoning, which results in models mainly relying on textual cues (Schwartz et al., 2019a). Malinowski and Fritz (2014) suggest Wu-Palmer similarity metric that calculates similarity based on the depth of two words based on the WordNet taxonomy (Miller, 1995). A different approach suggested in the VQA dataset focus only on brief, mostly 1-word answers (Antol et al., 2015). In this setup, the task turns into popular answers classification, alleviating many text-generation challenges. Notably, VQA requires 3 out of 10 annotators to agree on the answer, which is robust to inter-person variation. Still, accuracy ignores the reasoning process. Hudson and Manning (2019) propose GQA, which extends the accuracy metric and uses a scene graph for both question generation and evaluation. Following, Das et al. (2017) propose the VisDial dataset for the visual dialog task, which formulates multiple image-language interactions via a dialog. Concurrently, de Vries et al. (2017) propose GuessWhat, a goal-driven dialog dataset for object identification. Different from VQA and goal-driven dialogs, the VisDial answers are detailed and more human-like. For instance, in Fig. 1, the answer is "Can't tell...cloth", while a VQA answer would be "cloth". Therefore, metrics that require exact matching are no longer suitable. Instead, each question is accompanied with 100 candidate answers. Consequently, the metric has been shifted from accuracy to retrieval-based metrics, e.g., MRR and NDCG. Prior works focus on optimizing a single metric (Guo et al., 2019; Jiang et al., 2020; Hu et al., 2017; Gan et al., 2019). Differently, Murahari et al. (2020) attempt to optimize both metrics with a joint loss. Still, a dedicated single metric model is superior. Instead, we propose principals to ensemble two dedicated models, one for NDCG and one for MRR. Our approach allows most of the MRR and NDCG to be preserved simultaneously. Visual dialog models: Various approaches were proposed to solve the Visual Dialog task. Most of them focus on dialog history reasoning per interaction. Serban et al. (2017) propose history hierarchical encoding. Seo et al. (2017) introduce a memory network based on attention, which also addressed co-referential issues. Kottur et al. (2018) focus on visual co-reference. Jain et al. (2019) concatenate representations of all the cues (e.g., image, question, history, and caption) per candidate answer. Zheng et al. (2019) employ a graph structure learning. Schwartz et al. (2019b) propose a model, namely Factor Graph Attention (FGA), that lets all entities (e.g., question-words, image-regions, answer-candidate, and caption-words) interact to infer an attention map for each modality. An ensemble of five FGA models achieves the state-of-the-art MRR performance. However, FGA optimizes using the sparse annotations, i.e., the human-derived answer. Murahari et al. (2020) recently propose Large-Scale(LS) model, which pre-trains on related vision-language datasets, e.g., Conceptual Captions and Visual Question Answering(Sharma et al., 2018; Antol et al., 2015). Concurrently, Wang et al. (2020) leverage the pretrained BERT language models, and Nguyen et al. (2020) propose a lightweight Transformer that handles the interplay between many modalities. The three methods mentioned above finetune using the dense annotation (i.e., human assessment of all the candidates), resulting in a substantial improvement on the NDCG metric. Importantly, Murahari et al. find that finetuning a model for NDCG hurts MRR performance. This work demonstrates that re-ranking MRR model (e.g., FGA) and NDCG model (e.g., LS) with simple principles keeps most MRR and NDCG performance.

\section{Two-step Rank Ensemble}

The MRR metric depends on a single humanderived answer. Hence, given that this answer is ranked highly, the remaining candidates can be ranked according to the NDCG model. In the following, we describe two steps: (i) the MRR step responsible for preserving the human-derived rank 
high, and (ii) the NDCG step responsible for ranking the remaining candidates based on the NDCG model.

\subsection{Setup}

We are given a set of dialog questions $\left\{\left(q, \mathcal{C}_{q}\right)_{i}\right\}_{i=1}^{d}$, where $d$ is the dataset size, $q$ is a dialog question, and $\mathcal{C}_{q}=\left\{c_{q, j}\right\}_{j=1}^{100}$ are the corresponding candidates. The MRR metric, i.e., the inverse harmonic mean of rank, is defined as:

$$
\mathrm{MRR}=\frac{1}{d} \sum_{i=1}^{d} \frac{1}{r_{\mathrm{i}}},
$$

where $r_{i}$ is the rank of the human response for the $i$-th dialog question. The DCG, i.e., discounted cumulative gain over the $K$ correct answers, is defined as:

$$
\mathrm{DCG}_{\mathrm{K}}=\sum_{i=1}^{K} \frac{s_{i}}{\log _{2}(i+1)},
$$

where $s_{i}$ is a binary score, representing the fraction of annotators that marked the candidate at position as correct. We normalize by the ideal $\mathrm{DCG}_{\mathrm{K}}$ score $\left(\mathrm{IDCG}_{\mathrm{K}}\right)$, i.e., $\mathrm{NDCG}_{\mathrm{K}}=\frac{\mathrm{DCG}_{\mathrm{K}}}{\mathrm{IDCG}_{\mathrm{K}}}$. We denote the set of MRR models as $\mathcal{M}=\left\{M_{1}, \ldots, M_{n_{m}}\right\}$ where $n_{m}$ is the number of MRR models. Each MRR model is built by altering the initial conditions. We denote the NDCG model as $N$. We define an operator $\mathrm{T}(M, n, q)$ that returns the model $M$ 's top $n$ responses given a question $q$. Next, we describe the MRR step that aims to keep the MRR score.

\subsection{MRR step}

The purpose of the MRR step is to find a minimal candidate set $\mathcal{C}_{\mathrm{MRR}, q}$ that is likely to contain the human-derived answer given a question $q$. We build this set as a union of three sets, as follows:

$$
\mathcal{C}_{\mathrm{MRR}, q}=\mathcal{T}_{q} \cup \mathcal{N}_{q} \cup \mathcal{H}_{q},
$$

where $\mathcal{T}_{q}$ is a set of first ranked candidates according to MRR models, $\mathcal{N}_{q}$ is a set of high ranked candidates by both MRR and NDCG models, $\mathcal{H}_{q}$ is a set of high-certainty candidates agreed by all the MRR models. All sets are conditioned by the question $q$. In the following, we formally define those sets.

\subsubsection{High-certainty answers}

One of the most significant signals to be the humanderived answer is being a top MRR-model's answer. However, in many subjective questions, the
MRR model is not certain. We found that in those cases, the top answers often varies between different MRR models. Thus, to verify the top candidate's certainty, we require an agreement of MRRmodels. Let $q$ be a dialog question, we define the high-certainty set as follows:

$$
\mathcal{H}_{q}=\left\{c \mid\left(\forall M \in \mathcal{M} ; c \in \mathrm{T}\left(M, \rho_{h}, q\right)\right)\right\},
$$

where $\rho_{h} \in \mathbb{R}$ is an hyperparameter. Intuitively, a low $\rho_{h}$ results in higher certainty. We Next, we add the MRR-models' answer at first retrieval.

\subsubsection{Top answers}

The MRR metric prioritizes the first-ranked answer (see Eq.(1)). This property suits the nature of dia$\log$ models that reply with a single response. Consequently, we keep the first responses of the MRR models. Let $q$ be a dialog question, the top-answers set is defined as:

$$
\mathcal{T}_{q}=\left\{c \mid\left(\exists M \in \mathcal{M} ; c \in \mathrm{T}\left(M, \rho_{t}, q\right)\right)\right\},
$$

where $\rho_{t} \in \mathbb{R}$ is an hyperparameter. We note that $\rho_{t}$ should be low to maintain candidates' certainty. In the next step, we consider top NDCG candidates.

\subsubsection{NDCG-agreement answers}

When the NDCG model and the MRR model agree that a candidate is likely to be correct, it implies that both the NDCG and MRR metrics gain by ranking this candidate high. Thus, we want to rank it high. We note that the MRR set is ranked first, so we include these candidates in the MRR set. Let $q$ be a dialog question, the ndcg-agreement set is defined as:

$$
\mathcal{N}_{q}=\left\{c \mid \exists M \in \mathcal{M} ; c \in \mathrm{T}\left(N, \rho_{n}^{n}, q\right) \cap \mathrm{T}\left(M, \rho_{m}^{n}, q\right)\right\},
$$

where $\rho_{n}^{n}, \rho_{m}^{n} \in \mathbb{R}$ are hyperparameters that indicate relevancy to NDCG and MRR, respectively. I.e., as $\rho_{n n}$ increases, we may include more relevant candidates according to the NDCG model.

Up until this stage we have built a minimal set $C_{\mathrm{MRR}, q}$ that is likely to hold the human-derived answer. In the following we describe how we rank this set.

\subsubsection{MRR ranking}

Let $r_{M_{i}, c, q}$ denote the rank according to $M_{i} \in \mathcal{M}$ of candidate $c$ for a question $q$. We compute the MRR rank of candidate $c \in \mathcal{C}_{\mathrm{MRR}, q}$ via geometric mean: $r_{\mathrm{MRR}, c, q}=\prod_{i=1}^{n_{m}} r_{M_{i}, c, q}$. 


\begin{tabular}{|c|c|c|c|c|c|c|}
\hline Model & MRR $\uparrow$ & $R @ 1 \uparrow$ & $R @ 5 \uparrow$ & $\mathrm{R} @ 10 \uparrow$ & Mean $\downarrow$ & NDCG $\uparrow$ \\
\hline NMN (Kottur et al., 2018) & 58.80 & 44.15 & 76.88 & 86.88 & 4.81 & 58.10 \\
\hline NN (Zheng et al., 2019) & 61.37 & 47.33 & 77.98 & 87.83 & 4.57 & 52.82 \\
\hline CorefNMN (Kottur et al., 2018) & 61.50 & 47.55 & 78.10 & 88.80 & 4.40 & 54.70 \\
\hline RvA (Niu et al., 2019) & 63.03 & 49.03 & 80.40 & 89.83 & 4.18 & 55.59 \\
\hline HACAN (Yang et al., 2019) & 64.22 & 50.88 & 80.63 & 89.45 & 4.20 & 57.17 \\
\hline MReal - BDAI $¥$ (Qi et al., 2020) & 52.62 & 40.03 & 68.85 & 79.15 & 6.76 & 74.02 \\
\hline ReDAN (Gan et al., 2019) & 53.13 & 41.38 & 66.07 & 74.50 & 8.91 & 61.86 \\
\hline $\operatorname{ReDAN}^{\dagger}$ (Gan et al., 2019) & 53.74 & 42.45 & 64.68 & 75.68 & 6.64 & 64.47 \\
\hline DualVD (Jiang et al., 2020) & 63.23 & 49.25 & 80.23 & 89.70 & 4.11 & 56.32 \\
\hline DL-61 (Guo et al., 2019) & 62.20 & 47.90 & 80.43 & 89.95 & 4.17 & 57.32 \\
\hline DL-61 ${ }^{\dagger}$ (Guo et al., 2019) & 63.42 & 49.30 & 80.77 & 90.68 & 3.97 & 57.88 \\
\hline DAN (Kang et al., 2019) & 63.20 & 49.63 & 79.75 & 89.35 & 4.30 & 57.59 \\
\hline $\operatorname{DAN}^{\dagger}$ (Kang et al., 2019) & 64.92 & 51.28 & 81.60 & 90.88 & 3.92 & 59.36 \\
\hline LTMI (Nguyen et al., 2020) & 64.20 & 50.20 & 80.68 & 90.35 & 4.05 & 59.03 \\
\hline 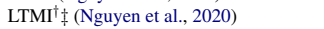 & 52.14 & 38.93 & 66.60 & 80.65 & 6.53 & 74.88 \\
\hline FGA (Schwartz et al., 2019b) & 63.75 & 49.58 & 80.97 & 88.55 & 4.51 & 52.12 \\
\hline $5 \times \mathrm{FGA}^{\dagger}$ (Schwartz et al., 2019b) & 69.37 & 55.65 & 86.73 & 94.05 & 3.14 & 57.29 \\
\hline LS(CE)* $\ddagger$ (Murahari et al., 2020) & 50.74 & 37.95 & 64.13 & 80.00 & 6.28 & 74.47 \\
\hline LS(CE+NSP)* $*$ (Murahari et al., 2020) & 63.92 & 50.78 & 79.53 & 89.60 & 4.28 & 68.08 \\
\hline LS* (Murahari et al., 2020) & 67.50 & 53.85 & 84.68 & 93.25 & 3.32 & 63.87 \\
\hline VD-BERT*怰 (Wang et al., 2020) & 51.17 & 38.90 & 62.82 & 77.98 & 6.69 & 75.35 \\
\hline $5 \mathrm{xFGA}+\mathrm{LS} * \dagger$ & 71.24 & 58.28 & 87.55 & 94.45 & 2.96 & 64.04 \\
\hline $5 \mathrm{xFGA}+\mathrm{LS}+\mathrm{LS}(\mathrm{CE})^{*} \dagger \ddagger$ & 68.78 & 55.72 & 85.02 & 93.55 & 3.26 & 69.22 \\
\hline Ours*tł & 70.41 & 58.18 & 83.85 & 90.83 & 3.66 & 72.16 \\
\hline \multicolumn{7}{|c|}{ Visual Dialog Challenge 2020 Leaderboard } \\
\hline LS & 68.79 & 55.20 & 86.15 & 93.88 & 3.12 & 63.34 \\
\hline VD-BERT & 51,84 & 39.91 & 63.45 & 78.56 & 6.57 & $\underline{75.92}$ \\
\hline MReaL Lab ( $3^{\text {rd }}$ Place $)$ & 64.12 & 50.81 & 80.03 & 90.92 & 3.83 & $\overline{75.70}$ \\
\hline SES-100M ( $2^{\text {nd }}$ Place) & 63.84 & 55.62 & 72.20 & 83.70 & 5.84 & 75.86 \\
\hline Ours ( $\left(1^{t l}\right.$ Place $)$ & 70.42 & 58.59 & 82.85 & 88.84 & 3.96 & 73.35 \\
\hline
\end{tabular}

Table 1: Performance on VisDial v1.0 test-std. (*) denotes the use of external knowledge. $(\dagger)$ indicates ensemble model, and $(\ddagger)$ signifies fine-tuning on the dense annotations. Shown are the MRR, NDCG, the mean rank of the human-derived answer, and the recall at a certain number of retrievals.

\begin{tabular}{|c|c|c|c|c|c|c|c|c|c|}
\hline $\mathcal{H}$ & $\mathcal{T}$ & $\mathcal{N}$ & $\overline{M R R \uparrow}$ & $\mathrm{R} @ 1 \uparrow$ & $\mathrm{R} @ 5 \uparrow$ & $\overline{R @ 10 \uparrow}$ & Mean $\downarrow$ & $\overline{\mathrm{NDCG} \uparrow}$ & $\left|\mathcal{C}_{\mathrm{MRR}}\right|$ \\
\hline$\checkmark$ & $x$ & $x$ & 70.83 & 58.87 & 84.32 & 90.67 & 3.67 & 74.32 & 2.34 \\
\hline$x$ & 1 & $x$ & 68.63 & 59.12 & 79.08 & 88.53 & 4.15 & 74.31 & 1.12 \\
\hline$x$ & $x$ & $s$ & 61.75 & 51.74 & 70.35 & 85.88 & 4.94 & 74.69 & 3.97 \\
\hline$x$ & $s$ & $\checkmark$ & 69.21 & 59.17 & 78.68 & 88.53 & 4.11 & 74.33 & 4.27 \\
\hline$s$ & $x$ & $s$ & 71.15 & 59.11 & 84.38 & 90.67 & 3.65 & 73.29 & 4.87 \\
\hline 1 & $s$ & $x$ & 71.06 & 59.15 & 84.49 & 90.78 & 3.64 & 72.98 & 2.39 \\
\hline 1 & 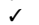 & $s$ & 71.26 & 59.18 & 84.62 & 90.78 & 3.62 & 73.23 & 4.91 \\
\hline \multicolumn{3}{|c|}{$\mathrm{LS}(\mathrm{CE})$} & 52.21 & 39.92 & 65.04 & 80.62 & 6.16 & 75.24 & 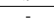 \\
\hline & 69.00 & 55.80 & 85,36 & 93.13 & 3.35 & $\overline{64.89}$ & . \\
\hline & & & 69.38 & 56.17 & 86.15 & 92.95 & 3.32 & 58.68 & 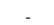 \\
\hline \multicolumn{3}{|c|}{$5 \mathrm{xFGA}+\mathrm{LS}$} & 72.25 & 59.20 & 88.55 & 94.52 & 2.84 & 65.34 & - \\
\hline \multicolumn{3}{|c|}{$5 \times \mathrm{xFG}+\mathrm{LS}+\mathrm{LS}(\mathrm{CE})$} & $\frac{2.25}{69.14}$ & $\frac{3.20}{56.79}$ & $\frac{0.05}{84.24}$ & 92.37 & $\frac{2.07}{3.43}$ & 73.78 & . \\
\hline
\end{tabular}

Table 2: MRR candidates set ablation analysis. Performance reported on the VisDialv1.0 val set.

\subsection{NDCG step}

In this step, we rank the remaining candidates $\mathcal{C}_{\mathrm{NDCG}, q}=\mathcal{C}_{q} \backslash \mathcal{C}_{\mathrm{MRR}, q}$. We assume the correct MRR answer is in $\mathcal{C}_{\text {MRR }}$. Thus, we rank the remaining candidates, according to the NDCG model via geometric mean: $r_{\mathrm{NDCG}, c, q}=\left(r_{N, c, q}\right)^{p} \cdot r_{M, c, q}$, where $M \in \mathcal{M}$ is the most accurate MRR model, and $p \in \mathbb{R}$ is a calibration hyperparameter which controls the trade-off between MRR and NDCG.

To conclude, let $q$ be a dialog question and $\mathcal{C}_{q}$ the corresponding candidates. We first find $\mathcal{C}_{\mathrm{MRR}, q}$, and rank the set according to $r_{\mathrm{MRR}, c, q}$. We then rank the remaining candidates, according to $r_{\mathrm{NDCG}, c, q} \cdot$

\section{Results}

We show our results on the VisDial v1.0 dataset, where 123,287 images are used for training, 2,000 images for validation, and 8,000 images for testing (Das et al., 2017). Each image is associated with ten questions, and each question has 100 cor-

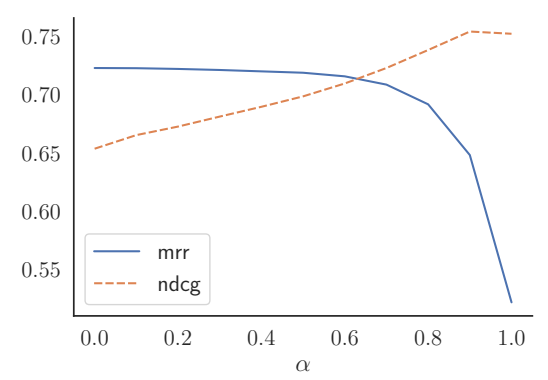

Figure 2: Performance of a naïve score ensemble of the MRR model and the NDCG model on the VisDialv1.0 val set. We calibrate the importance of each model with a scalar $\alpha$.

responding answer candidates. We use two MRR models (i.e., $n_{m}=2$ ), FGA (Schwartz et al., $2019 \mathrm{~b}$ ) and an ensemble of LS (Murahari et al., 2020) with FGA. We use LS(CE) as the NDCG model. We set $\rho_{h}=3, \rho_{t}=1, \rho_{n}^{n}=5, \rho_{m}^{n}=10$, and $p=3$. We tune these parameters using the validation set.

Comparison to state-of-the-art: In Tab. 1 we compare our method to naïve ensembles and previous baselines. We first ensemble the LS's output with the FGA's output. By combining them, we achieve the new MRR state-of-the-art $(71.24 \%$ vs. $69.37 \%$ ). Next, we build a naïve ensemble of the MRR model and the NDCG model. We do so by adding the MRR ensemble scores (denoted by $\mathcal{S}_{M}$ ) and LS(CE) scores (denoted by $\mathcal{S}_{N}$ ), as follows: $\alpha \cdot \mathcal{S}_{M}+(1-\alpha) \mathcal{S}_{N}$, where $\alpha \in \mathbb{R}$ calibrates the trade-off between MRR and NDCG performance. We show in Fig. 2 an analysis of different $\alpha$ values on the validation set. In Tab. 1, we report results for $\alpha=0.8$. Our two-step method outperforms the MRR $(70.41 \%$ vs. $68.78 \%)$ and NDCG $(72.16 \%$ vs. $69.22 \%$ ) metrics, despite lacking the output scores and only requiring rankings.

We also compare our approach to previous baselines. Most methods use the sparse annotations, i.e., the human-derived answer, while MReal-BDAI, VD-BERT, and LS(CE) finetune using the dense annotations. Finetuning with the dense annotations tremendously boosts the NDCG performance but loses MRR performance. The MRR performance decline can be attributed to NDCG being biased toward uncertain answers. We also note that LS leverages large-scale image-text corpora. LS(CE+NSP) optimizes both the dense and sparse annotations but still suffers from a performance drop compared to metric-dedicated LS models, i.e., MRR $(63.92 \%$ vs. $67.50 \%)$ and NDCG (68.08\% vs. $74.47 \%)$. Unlike the method mentioned above, our method re-rank the candidates based on two distinct models, with 

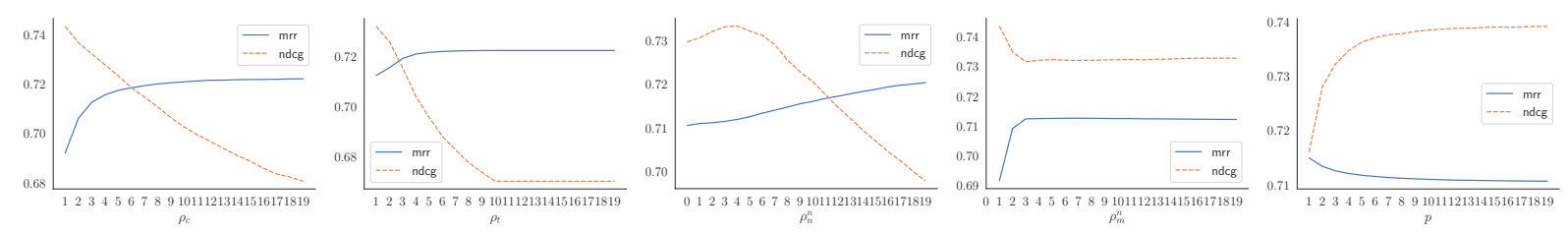

Figure 3: MRR and NDCG scores for different hyperparameter values.

two distinct steps, to keep the human-derived answer high. In doing so, we achieve a good MRR performance $(70.41 \% v s .71 .24 \%)$, yet notably with limited NDCG drop (72.25\% vs. $75.35 \%)$. This property comes in handy in the recent Visual Dialog challenge, where the winners were picked based on both the NDCG and MRR evaluation metrics. Our method performs well on both metrics simultaneously and won the challenge.

Ablation analysis: The MRR candidate set consists of different subsets. In Tab. 2 we show the influence of each of subset independently on the retrieval metrics. Further, omitting a subset harms the performance, i.e. each component is essential to preserve both the MRR and NDCG metrics. We also report the average size of the MRR-candidate set, and the validation performance of the MRR model (i.e., 5xFGA) and the NDCG model (i.e., $\mathrm{LS}(\mathrm{CE})$ ). In addition we provide the results of the MRR ensemble, and the naïve NDCG and MRR ensemble for $\alpha=0.8$.

In Fig. 3, we examine how the NDCG and MRR metrics are affected by modifying one hyperparameter while maintaining the others. On the first figure from the left, we alter $\rho_{c}$. The higher $\rho_{c}$, we require higher agreement between the MRR models, resulting in higher certainty for elements in the MRR set. Because the MRR models are responsible for the MRR set ranking, an MRR set that is too large hurts the NDCG metric. For the same reason, in the second image from the left, increasing $\rho_{t}$, significantly harms the NDCG performance. In the third figure from the left, we show that considering more candidates that both NDCG and MRR models agree upon (i.e., increasing $\rho_{n}^{n}$ ) helps both metrics' performance. However, adding too many candidates harms the NDCG metric. In the fourth image from the left, we show that the performance remains stable when $\rho_{m}^{n}$ is larger than three. Last, on the fifth image from the left, we show the effect of changing $p$, which calibrates the trade-off between MRR and NDCG during the NDCG ranking step.

Qualitative analysis: In Fig. 4, we show two sample visual dialogs from test-std. For each sample,

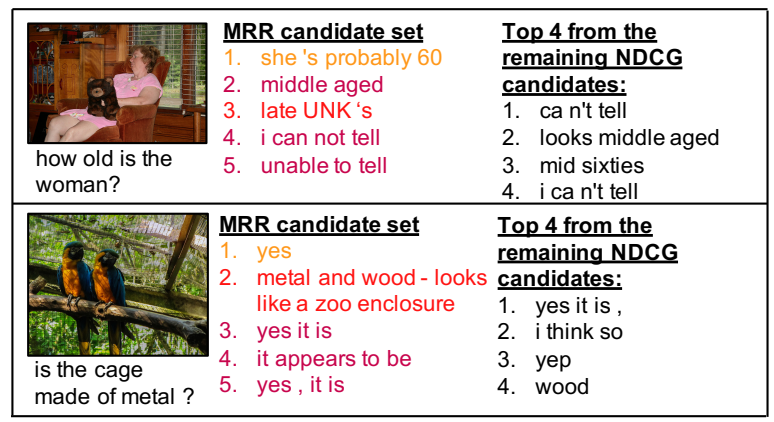

Figure 4: An illustration of two visual dialog samples. Each sample includes the MRR candidate set and four answers from the remaining NDCG candidates. We find that the MRR candidate set has more certain answers. We colorize the highcertainty candidates $(\mathcal{H})$ with orange, the $N D C G$-agreement candidates $(\mathcal{N})$ with purple, and the top-answers subset $(\mathcal{T})$ with red. Note, if a candidate belongs to more than one set, we sketch the colors in the following order: orange $\rightarrow$ red $\rightarrow$ purple.

we provide the ranked MRR candidate set and the next 4 NDCG candidates. The analysis reveals the answers' ambiguity and that the MRR candidate set mostly consists of certain responses. In addition, we highlight the candidates within each MRR candidate subset with different colors. Additional samples can be found in the appendix.

\section{Conclusions}

We describe a non-parametric method to ensemble the candidate ranks of two strong MRR and NDCG models into a single ranking that excels on both NDCG and MRR. Intuitively, we use the MRRmodel for non-ambiguous questions with certain answers. The dense-annotations cue is more applicable in ambiguous questions than the sparse annotations. Thus, in the case of low certainty, our method relies almost entirely on the NDCG model. We hope the proposed principles can guide the community towards a parametric model that can employ answers' semantics to measure certainty.

\section{Acknowledgements}

We thank Yftah Ziser, Itai Gat, Alexander Schwing and Tamir Hazan for useful discussions. 


\section{References}

Stanislaw Antol, Aishwarya Agrawal, Jiasen Lu, Margaret Mitchell, Dhruv Batra, C. Lawrence Zitnick, and Devi Parikh. 2015. VQA: Visual Question Answering. In ICCV.

Abhishek Das, Satwik Kottur, Khushi Gupta, Avi Singh, Deshraj Yadav, José MF Moura, Devi Parikh, and Dhruv Batra. 2017. Visual dialog. In CVPR.

Zhe Gan, Yu Cheng, Ahmed EI Kholy, Linjie Li, Jingjing Liu, and Jianfeng Gao. 2019. Multi-step reasoning via recurrent dual attention for visual dia$\log$. ACL.

Donald Geman, Stuart Geman, Neil Hallonquist, and Laurent Younes. 2015. Visual turing test for computer vision systems. NAS.

Dalu Guo, Chang Xu, and Dacheng Tao. 2019. Imagequestion-answer synergistic network for visual dialog. In $C V P R$.

Ronghang $\mathrm{Hu}$, Jacob Andreas, Marcus Rohrbach, Trevor Darrell, and Kate Saenko. 2017. Learning to reason: End-to-end module networks for visual question answering. ICCV.

Drew A Hudson and Christopher D Manning. 2019. Gqa: A new dataset for real-world visual reasoning and compositional question answering. In $C V P R$.

Unnat Jain, Svetlana Lazebnik, and Alexander Schwing. 2019. Two can play this game: Visual dialog with discriminative question generation and answering. CVPR.

Xiaoze Jiang, Jing Yu, Zengchang Qin, Yingying Zhuang, Xingxing Zhang, Yue Hu, and Qi Wu. 2020. Dualvd: An adaptive dual encoding model for deep visual understanding in visual dialogue. $A A A I$.

Gi-Cheon Kang, Jaeseo Lim, and Byoung-Tak Zhang. 2019. Dual attention networks for visual reference resolution in visual dialog. $A C L$.

Satwik Kottur, José MF Moura, Devi Parikh, Dhruv Batra, and Marcus Rohrbach. 2018. Visual coreference resolution in visual dialog using neural module networks. In $E C C V$.

Chia-Wei Liu, Ryan Lowe, Iulian V Serban, Michael Noseworthy, Laurent Charlin, and Joelle Pineau. 2016. How not to evaluate your dialogue system: An empirical study of unsupervised evaluation metrics for dialogue response generation. arXiv preprint arXiv:1603.08023.

Mateusz Malinowski and Mario Fritz. 2014. A multiworld approach to question answering about realworld scenes based on uncertain input. In NIPS.

George A Miller. 1995. Wordnet: a lexical database for english. Communications of the ACM.
Vishvak Murahari, Dhruv Batra, Devi Parikh, and Abhishek Das. 2020. Large-scale pretraining for visual dialog: A simple state-of-the-art baseline. ECCV.

Van-Quang Nguyen, Masanori Suganuma, and Takayuki Okatani. 2020. Efficient attention mechanism for handling all the interactions between many inputs with application to visual dialog. ECCV.

Yulei Niu, Hanwang Zhang, Manli Zhang, Jianhong Zhang, Zhiwu Lu, and Ji-Rong Wen. 2019. Recursive visual attention in visual dialog. In $C V P R$.

Jiaxin Qi, Yulei Niu, Jianqiang Huang, and Hanwang Zhang. 2020. Two causal principles for improving visual dialog. CVPR.

Idan Schwartz, Alexander G Schwing, and Tamir Hazan. 2019a. A simple baseline for audio-visual scene-aware dialog. In CVPR.

Idan Schwartz, Seunghak Yu, Tamir Hazan, and Alexander G Schwing. 2019b. Factor graph attention. In $C V P R$.

Paul Hongsuck Seo, Andreas Lehrmann, Bohyung Han, and Leonid Sigal. 2017. Visual reference resolution using attention memory for visual dialog. In NIPS.

Iulian Vlad Serban, Alessandro Sordoni, Ryan Lowe, Laurent Charlin, Joelle Pineau, Aaron C Courville, and Yoshua Bengio. 2017. A hierarchical latent variable encoder-decoder model for generating dialogues. In $A A A I$.

Piyush Sharma, Nan Ding, Sebastian Goodman, and Radu Soricut. 2018. Conceptual captions: A cleaned, hypernymed, image alt-text dataset for automatic image captioning. In $A C L$.

Harm de Vries, Florian Strub, Sarath Chandar, Olivier Pietquin, Hugo Larochelle, and Aaron C Courville. 2017. Guesswhat?! visual object discovery through multi-modal dialogue. In CVPR.

Yue Wang, Shafiq Joty, Michael R Lyu, Irwin King, Caiming Xiong, and Steven CH Hoi. 2020. Vd-bert: A unified vision and dialog transformer with bert. EMNLP.

Tianhao Yang, Zheng-Jun Zha, and Hanwang Zhang. 2019. Making history matter: History-advantage sequence training for visual dialog. In ICCV.

Zilong Zheng, Wenguan Wang, Siyuan Qi, and SongChun Zhu. 2019. Reasoning visual dialogs with structural and partial observations. In $C V P R$.

\section{A Qualitative Analysis}

In the following, we show additional 200 randomly picked visual dialog samples from test-std. For each sample, we provide the ranked MRR candidate set and the next 10 NDCG candidates. We 
colorize the high-certainty candidates $(\mathcal{H})$ with orange, the $N D C G$-agreement candidates $(\mathcal{N})$ with purple, and the top-answers subset $(\mathcal{T})$ with red. Note, if a candidate belongs to more than one set, we sketch the colors in the following order: orange $\rightarrow$ red $\rightarrow$ purple.

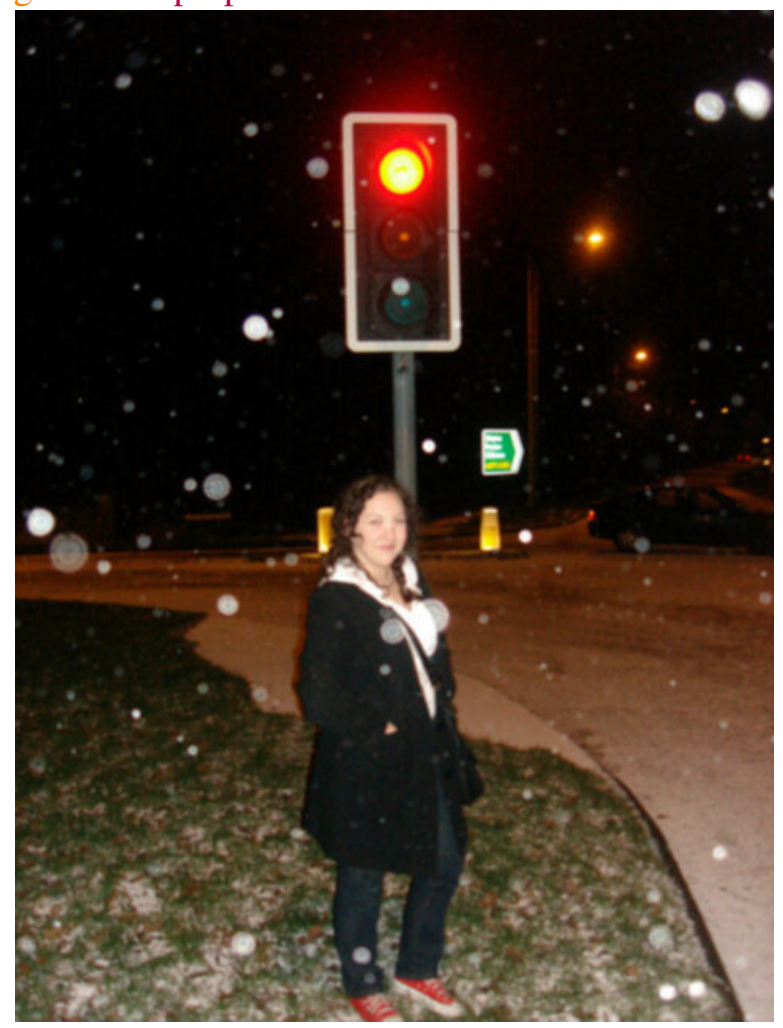

Question:what color is the light?

\section{MRR candidate set}

1. red

2. green

3. the light is currently green

4. the lights are n't visible

Top 10 from the remaining NDCG candidates

1. yellow

2. not sure

3. i ca n't tell

4. it 's kinda dark out so i am unsure

5. i can not tell

6. ca n't tell

7. black and red

8. UNK
9. black

10. it 's dark

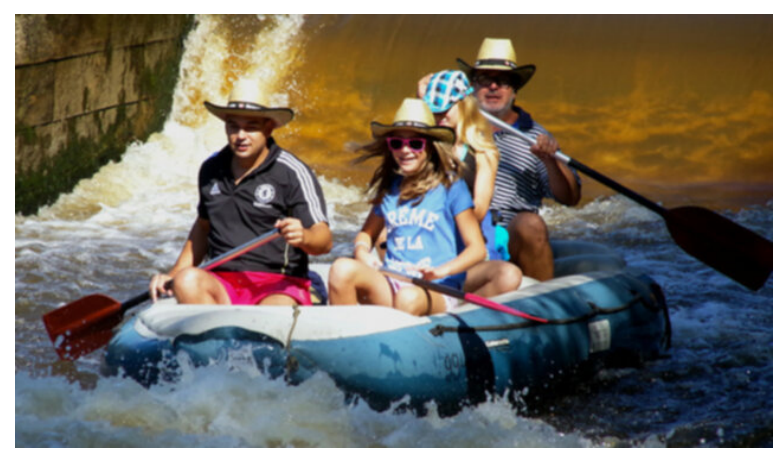

Question:what ethnicity do they appear to be ? MRR candidate set

1. all white

2. white

3. i can not tell

4. ca n't tell

5. not sure

Top 10 from the remaining NDCG candidates

1. black

2. i ca n't tell

3. both have brown hair

4. there are 2 teens, and 1 adult

5. i think like 40 's maybe

6. maybe younger seniors

7. dark brown

8. no, the tv is not that visible

9. 1 looks older the other is quite young

10. she appears to be caucasian

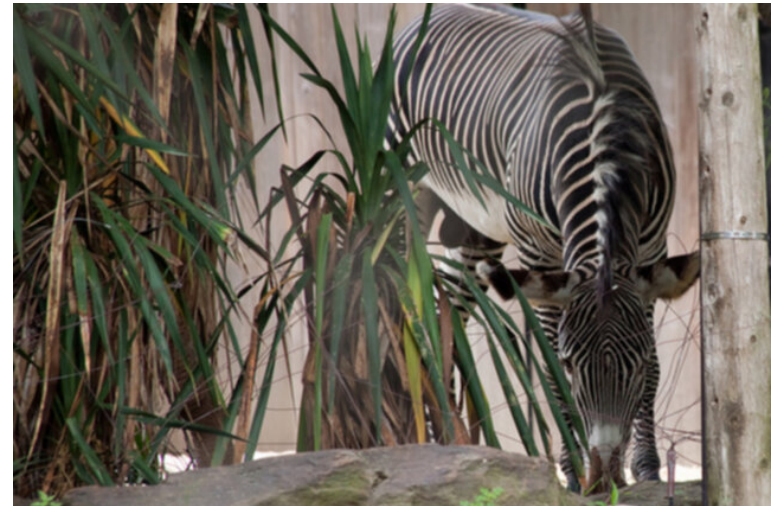

Question:are there any other animals? MRR candidate set 
1. no

2. not that i see

3. nope

4. not that i can see

\section{Top 10 from the remaining NDCG candidates}

1. no other animals

2. 0 that i can see

3. 0

4. 0 visible

5. lol, no

6. non

7. i do n't think so

8. i can not see

9. no , only animals

10. i do not see any animals

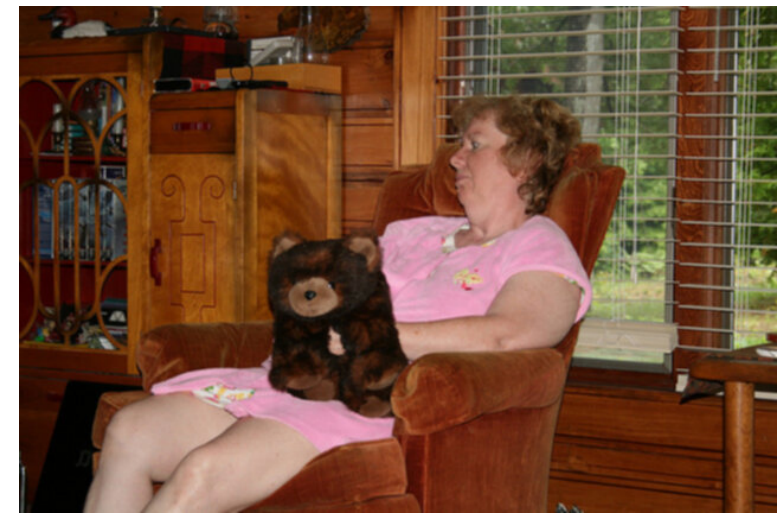

Question:how old is the woman?

MRR candidate set

1. she 's probably 60

2. middle aged

3. late UNK's

4. i can not tell

5. unable to tell

\section{Top 10 from the remaining NDCG candidates}

1. ca n't tell

2. looks middle aged

3. mid sixties
4. i ca n't tell

5. late 40 's

6. about middle aged maybe 40 something

7. $70 \mathrm{~s}$

8. not sure

9. about 30

10. at least $30 \mathrm{~s}$

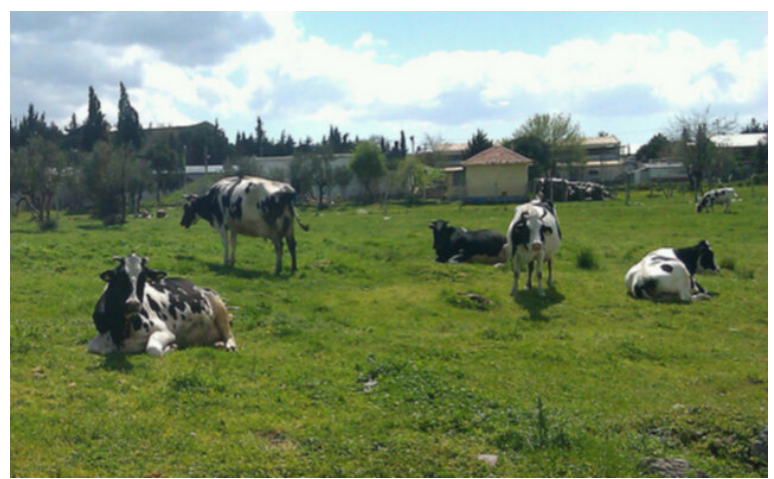

Question:do they all have any spots on them ? MRR candidate set

1. yes, all but 1 has spots

2. no

3. yes

4. not that $\mathrm{i}$ can see

5. it 's too hard to tell

6. nope

Top 10 from the remaining NDCG candidates

1. i do n't think so

2. i can not tell

3. very few

4. some do, yes

5. not really

6. some do

7. can not tell

8. i ca n't tell

9. ca n't tell

10. 0 


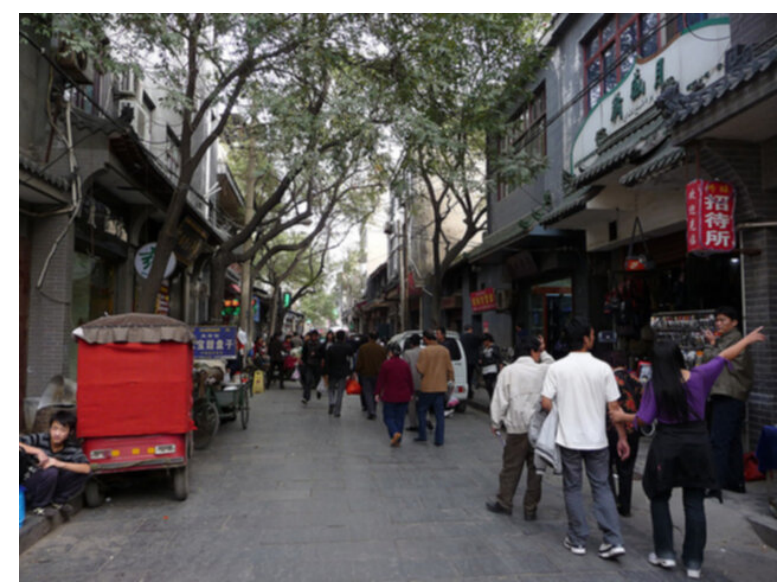

Question:see any store names ?

MRR candidate set

1. no

2. yes

3. i can not tell

4. unable to tell

5. not that i can tell

6. not that $i$ can see

Top 10 from the remaining NDCG candidates

1. nope

2. not really

3. i ca n't see it

4. not sure

5. i ca n't tell

6. ca n't tell

7. probably just 1

8. not at all

9. yes 1

10. i do n't think so

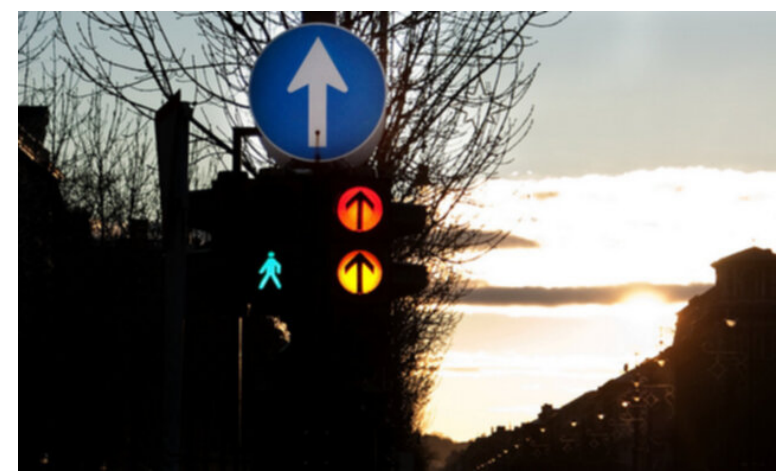

Question:what color is their hair?

MRR candidate set
1. i can not tell

2. black

3. ca n't see them

4. ca n't tell

5. it doesn ' t have hair it 's a light

6. i ca n't tell

\section{Top 10 from the remaining NDCG candidates}

1. not sure

2. dark is all i can tell

3. brown i believe

4. hard to tell, but it looks very blackened

5. brown

6. it looks brown

7. no people

8. dark brown

9. it is dark brown

10. it 's brown

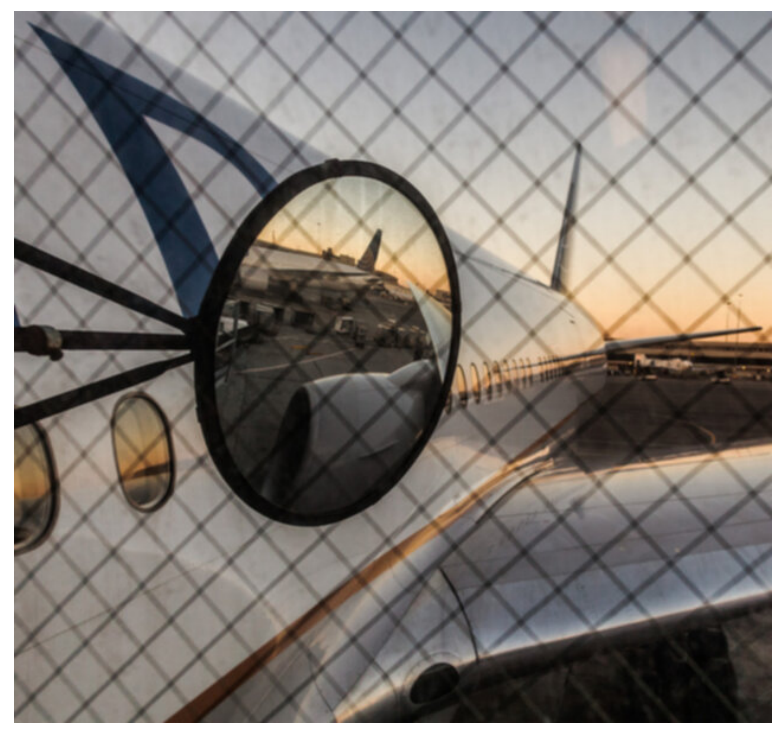

Question:is it daytime or night time?

MRR candidate set

1. daytime

2. day

3. it appears to be daytime

4. it is daytime 
5. it looks like it 's daytime

6. it 's daytime

\section{Top 10 from the remaining NDCG candidates}

1. it is day time

2. day time

3. it is day

4. it is during the day

5. hard to tell probably day

6. looks like early morning

7. daytime maybe dusk

8. hello, fellow turkey : yes, it is day time

9. in the even or morning

10. i ca n't tell

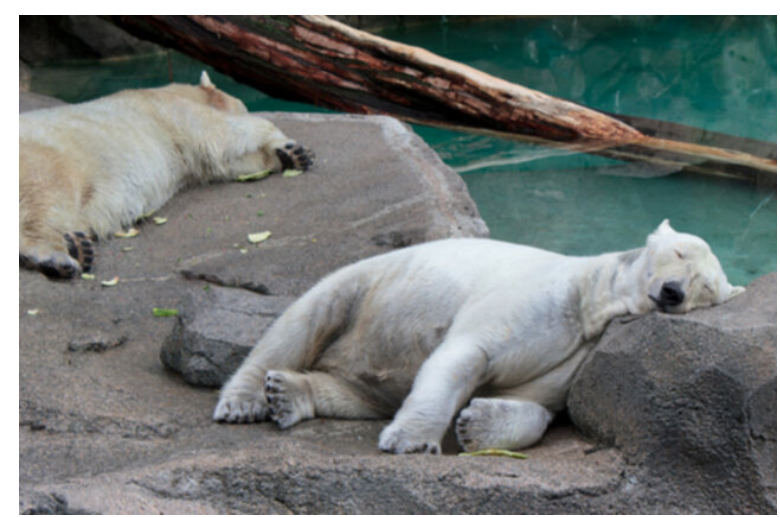

Question:is this photo in color?

MRR candidate set

1. yes

2. yes it is

3. yes, it is

4. yes it is in color

5. yes, it's in color

\section{Top 10 from the remaining NDCG candidates}

1. yes the picture is in color

2. yes in color

3 . it is

4. it is in color

5. it's in color
6. yes, it 's very colorful!

7. i think so

8. looks like it

9. it is, but there is not much color in the photo due to the objects

10. yes it is well lit

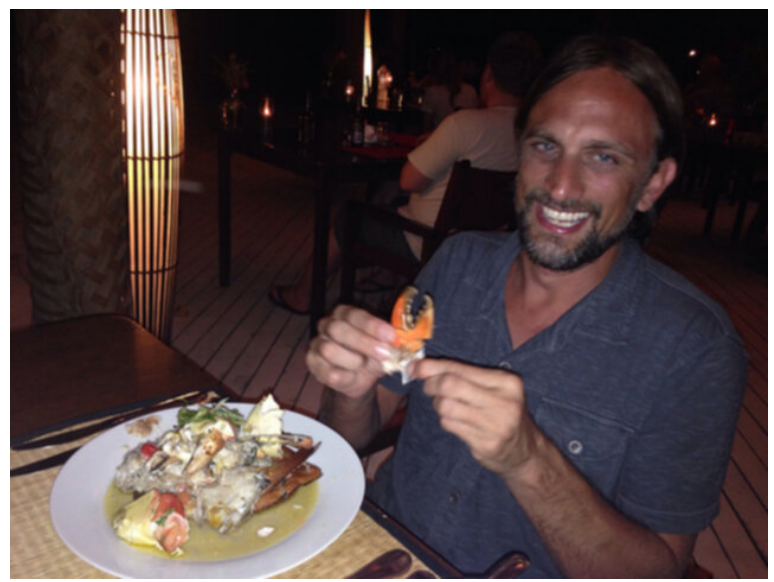

Question:what are the vegetables he has? MRR candidate set

1. salmon and greens, maybe spinach

2. it looks like greens, hard to tell

3. ca n't really tell

4. i can not tell

5. i ca n't tell

6. ca n't tell

\section{Top 10 from the remaining NDCG candidates}

1. not sure

2. i 'm not a $100 \%$ sure, look like it

3. yes

4. not that i can see

5. can not see grass

6. green

7. plate

8. looks old

9. i do n't think so

10. nope 


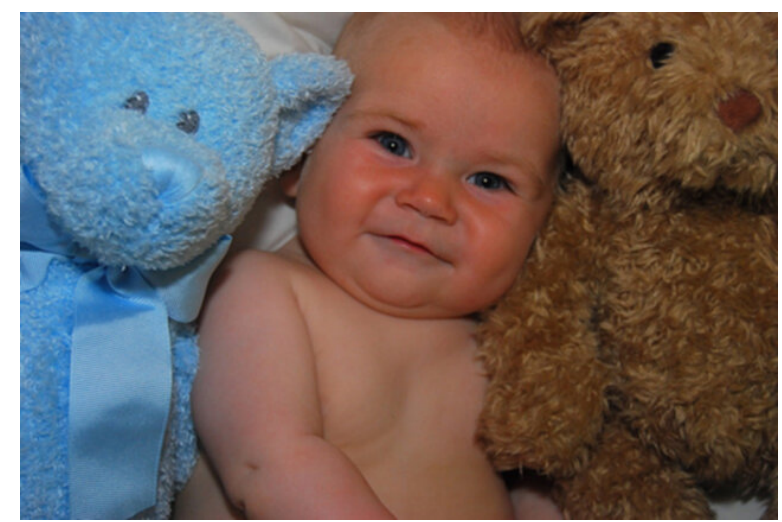

Question:are they all facing the camera ?

MRR candidate set

1. yes

2. no

3. slightly

4. yes they are

5. nope

Top 10 from the remaining NDCG candidates

1. not really

2. i don' $t$ think so

3. yes they all are

4. yes i believe so

5. no side view

6. i do n't think so

7. i think so

8. all but 1

9. not all of them

10. not that i can see

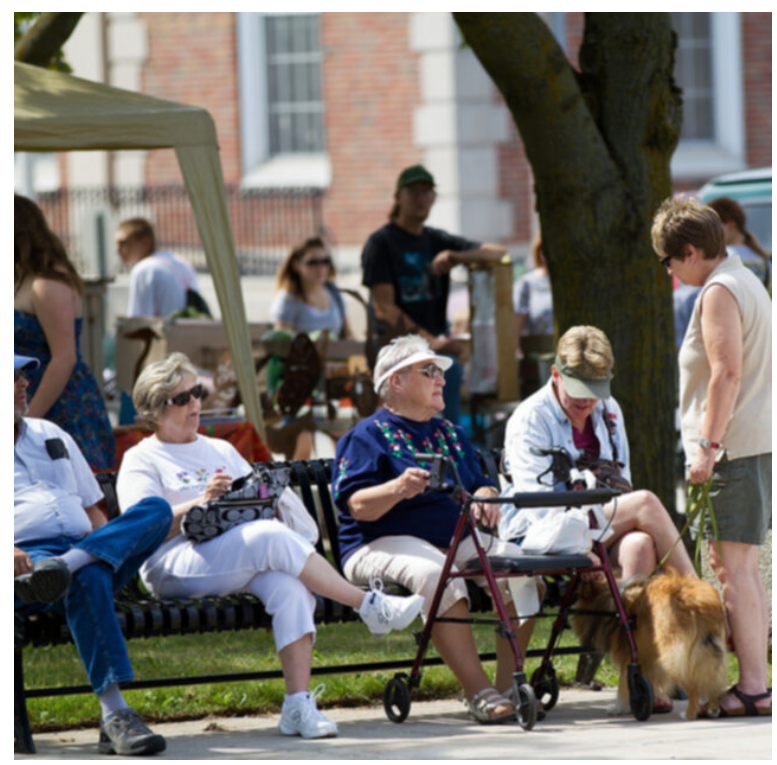

Question:is it a poodle?

MRR candidate set

1. no

2. i do n't think so

3. nope

\section{Top 10 from the remaining NDCG candidates}

1. does not look like it

2. i ca n't tell

3. i can not tell

4. not sure

5. i can not say

6. ca n't tell

7. not that $i$ can see

8. i do n't think so, but it 's hard to tell

9. i 'm not sure what that is

10. no i am unable to tell 


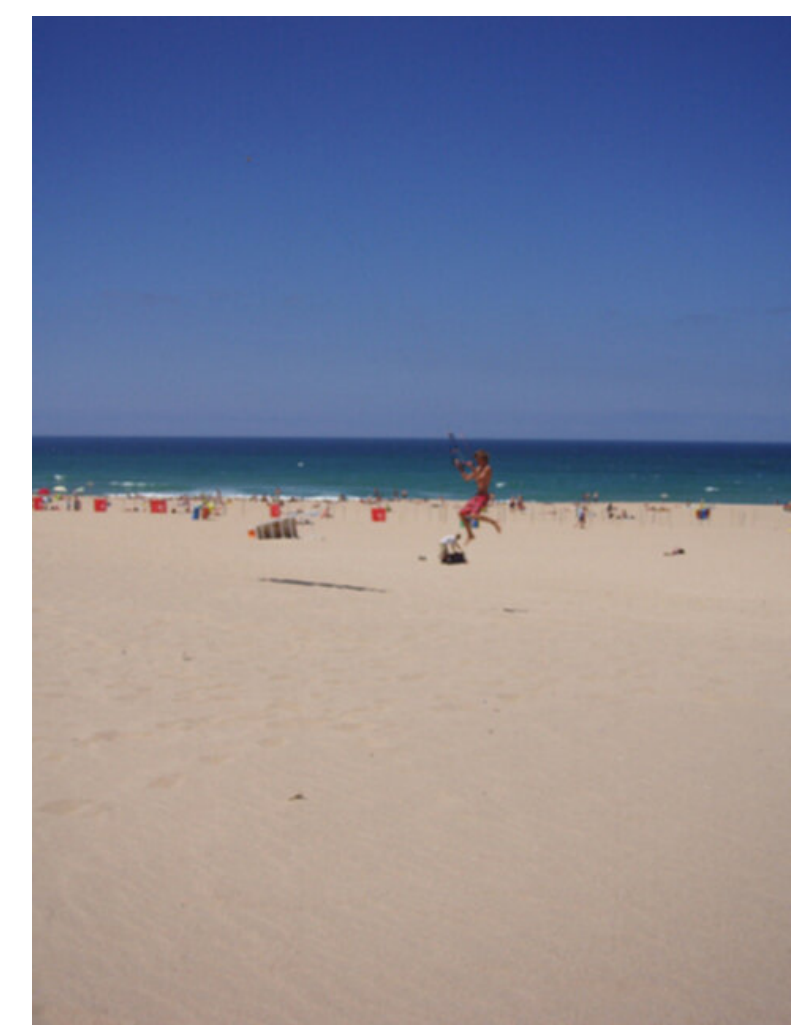

Question:how old is the man?

MRR candidate set

1. i can not tell

2. can not tell

3. unable to tell

4. ca n't see his face

5. i ca n't tell

6. ca n't tell

Top 10 from the remaining NDCG candidates

1. i ca n't tell i ca n't see their face

2. twenties maybe, hard to tell

3. hard to tell but i would say mid 20 's

4. hard to say but i guess around thirty

5. not sure

6. i can not see their face

7. maybe 25 ish

8. he looks to be about 30ish

9. it 's hard to tell , it 's from a distance

10. he looks to be maybe 20 or so

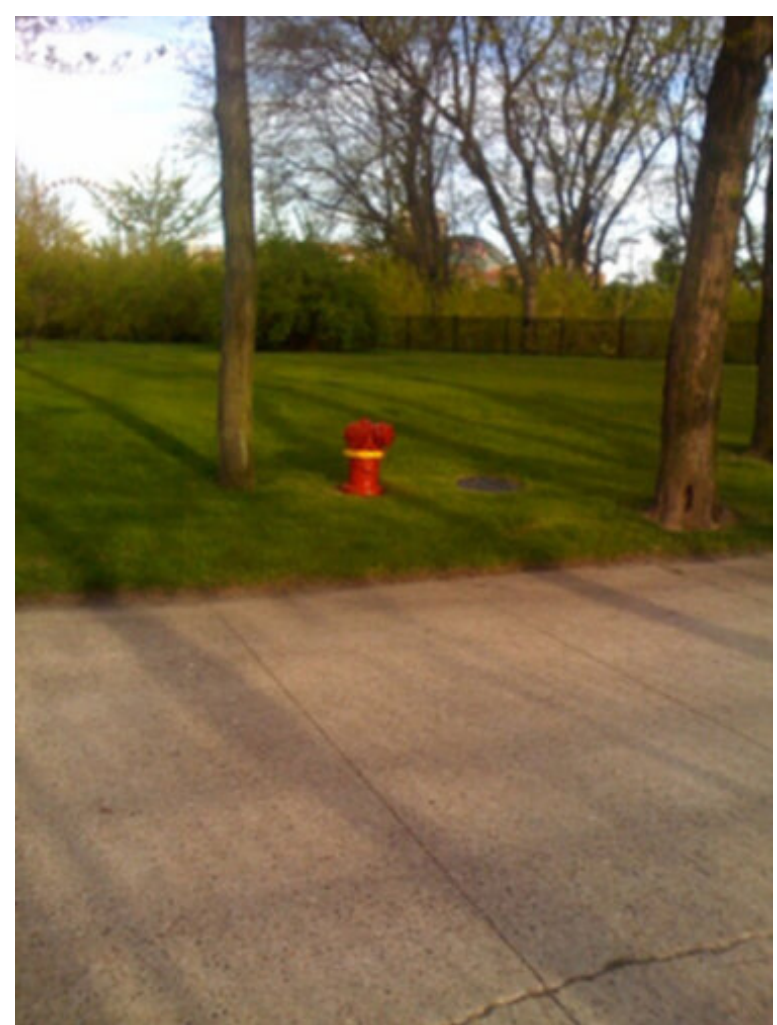

Question:is there anything near it? MRR candidate set

1. no

2. grass and tree 's

3. nothing

4. not that $i$ can see

5. nope

6. ca n't see anything

Top 10 from the remaining NDCG candidates

1. yes

2. i do n't think so

3. i don' t see any

4. not really

5. you can see a pitcher and maybe a wall

6. a few things

7. no, it 's just dirt

8. in the distance

9. no but it has a lot of stuff on it

10. not sure there may be, but it does n't show enough to tell 


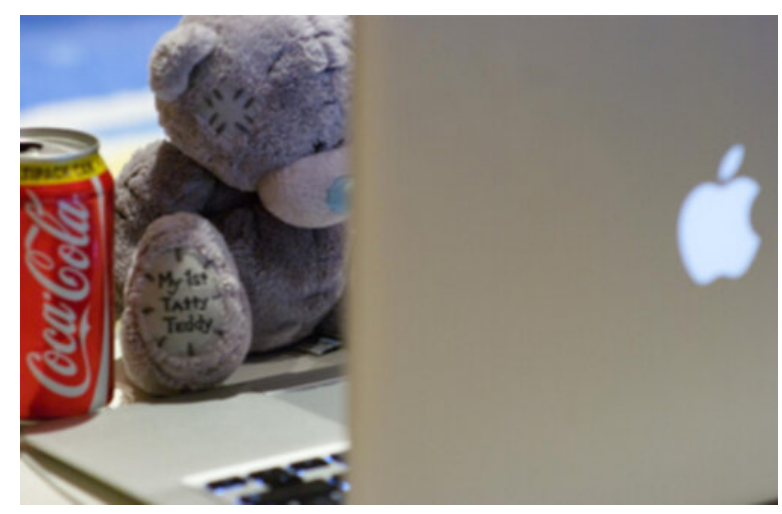

Question:is there anything on the wallpaper of the laptop ?

\section{MRR candidate set}

1. no

2. nope

3. not that i can see

\section{Top 10 from the remaining NDCG candidates}

1. no there is not

2. i can not tell

3. i do n't see any

4. ca n't tell

5. i ca n't tell

6. 0

7. i do n't think so

8. just a screen over it

9. not that $\mathrm{i}$ can UNK for sure

10. not sure

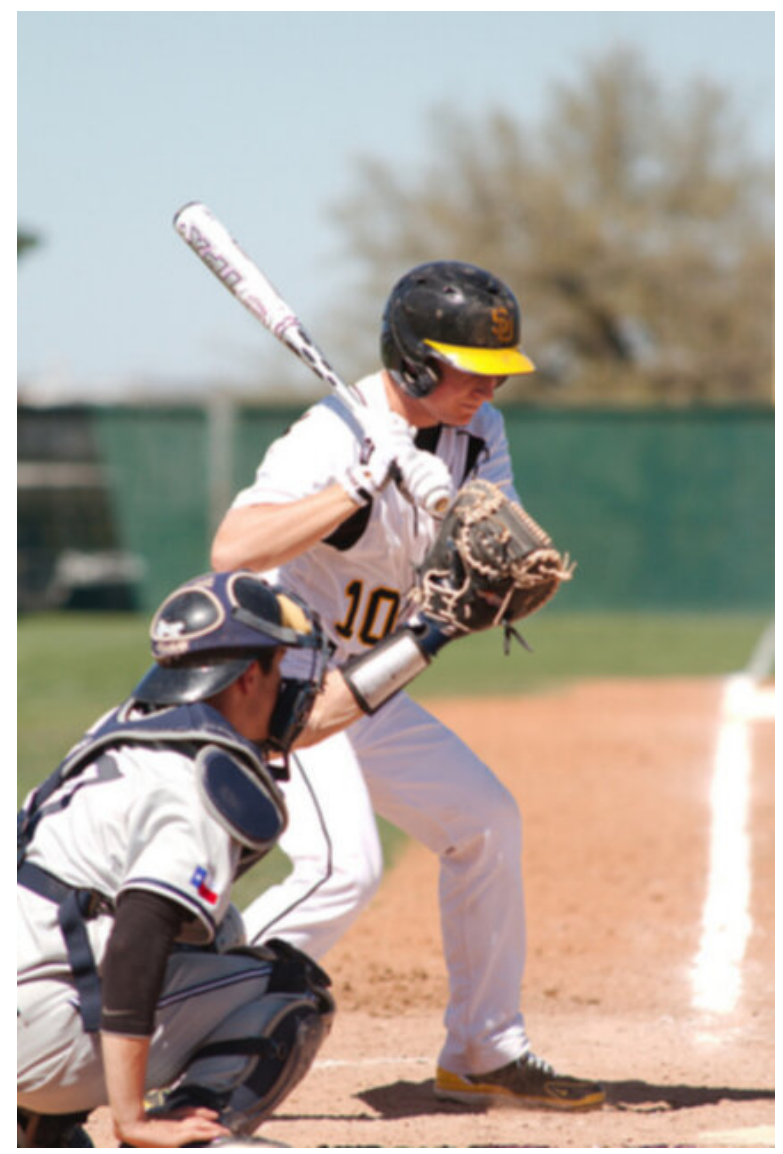

Question:is it date stamped?

MRR candidate set

1. no

2. no it's not

3. no it is n't

4. nope

5. no, it's not

Top 10 from the remaining NDCG candidates

1. not that i can see

2. i do n't think so

3. i do n't know

4. do n't know

5. i ca n't tell

6. ca n't tell

7. not sure

8. i can not tell

9. not readable

10. nah 


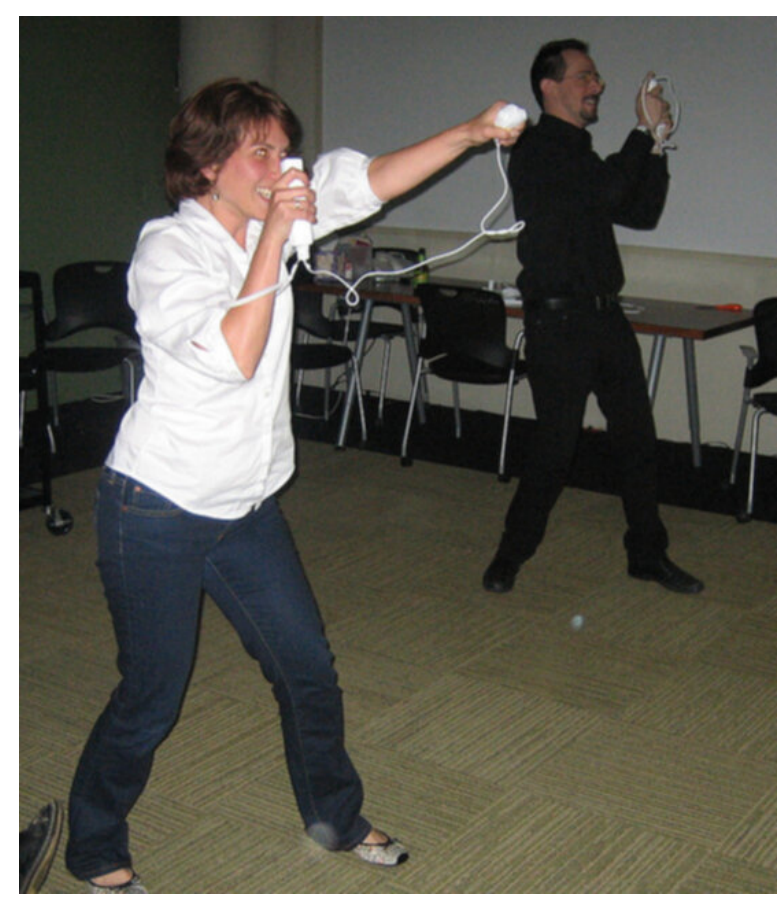

Question:is this inside?

MRR candidate set

1. yes

2. yes it is

3. yes, it is

4. yes i think so

Top 10 from the remaining NDCG candidates

1. yes, it seems to be

2. i think so, yes

3. this is inside

4. it seems to be

5. it is indoors

6. i believe so

7. i think so

8. looks like it

9. no

10. ues

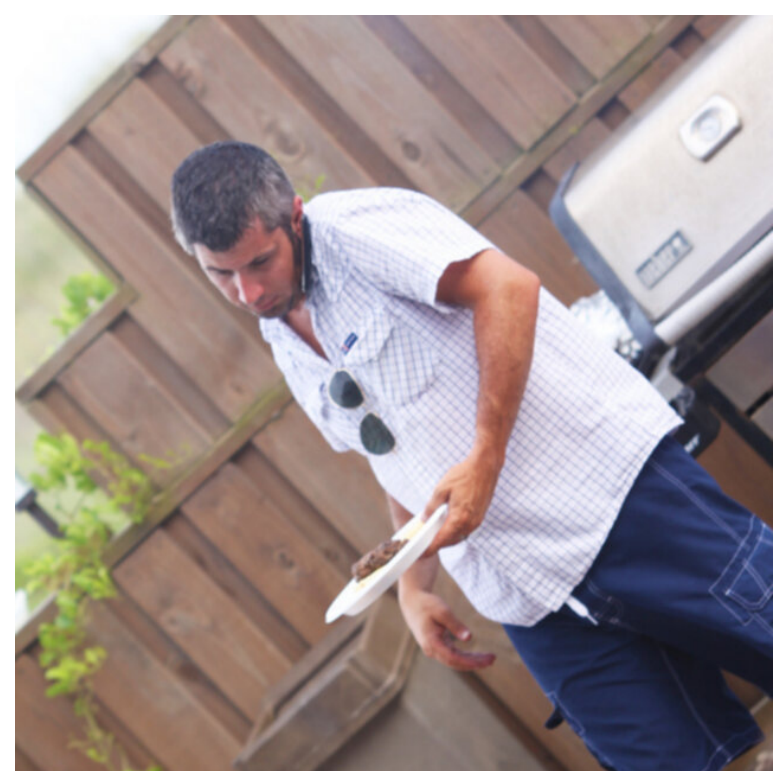

Question:does the food look appetizing ? MRR candidate set

1. yes

2. no

3. not really

4. not at all

5. nope

Top 10 from the remaining NDCG candidates

1. yes it does

2. no, he does n't

3. yes, it does

4. i can not tell

5. ca n't tell

6. a little

7. not that i can see

8. sort of

9. kinda

10. i ca n't tell 


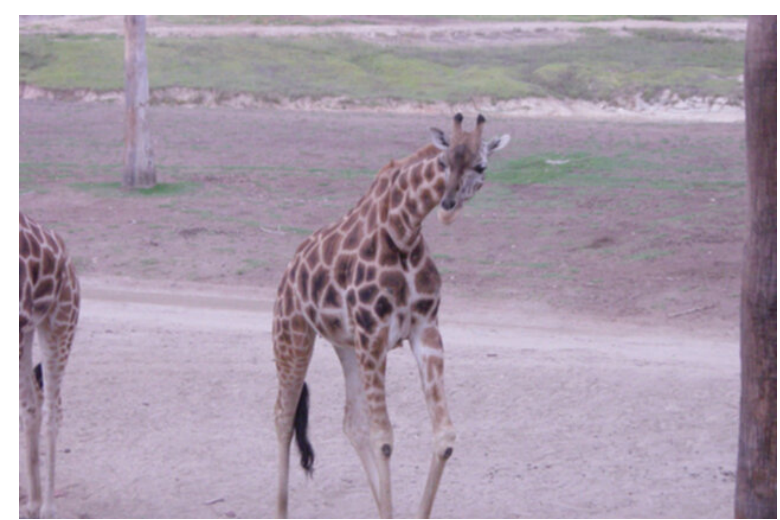

Question:is the image in color ?

MRR candidate set

1. yes

2. yes it is

3. yes, it is in color

4. yes it is in color

5. yes, it 's in color

6. yes, the photo is in color

\section{Top 10 from the remaining NDCG candidates}

1. yes, it is

2. yes the photo is in color

3. yes, full color photo

4. yes it 's color

5. it is

6. yes in color

7. it is yes

8. yess

9. yea

10. yyes

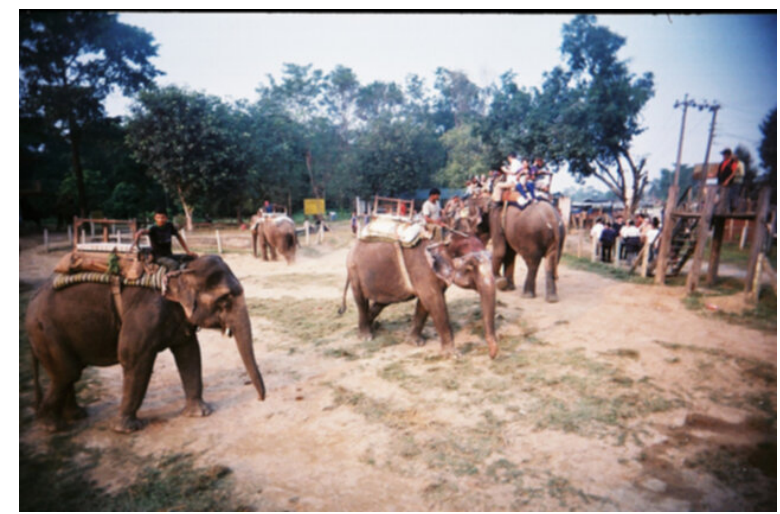

Question:is it daytime?

MRR candidate set
1. yes

2. yes it is

3. yes, it is daytime

4. yes it is daytime

5. yes, it is

Top 10 from the remaining NDCG candidates

1. it is

2. yes, it looks to be

3. it is daytime

4. yup

5. it is day time

6. yes, i think so

7. i believe so

8. yes it is, it looks sunny

9. daytime

10. i think so

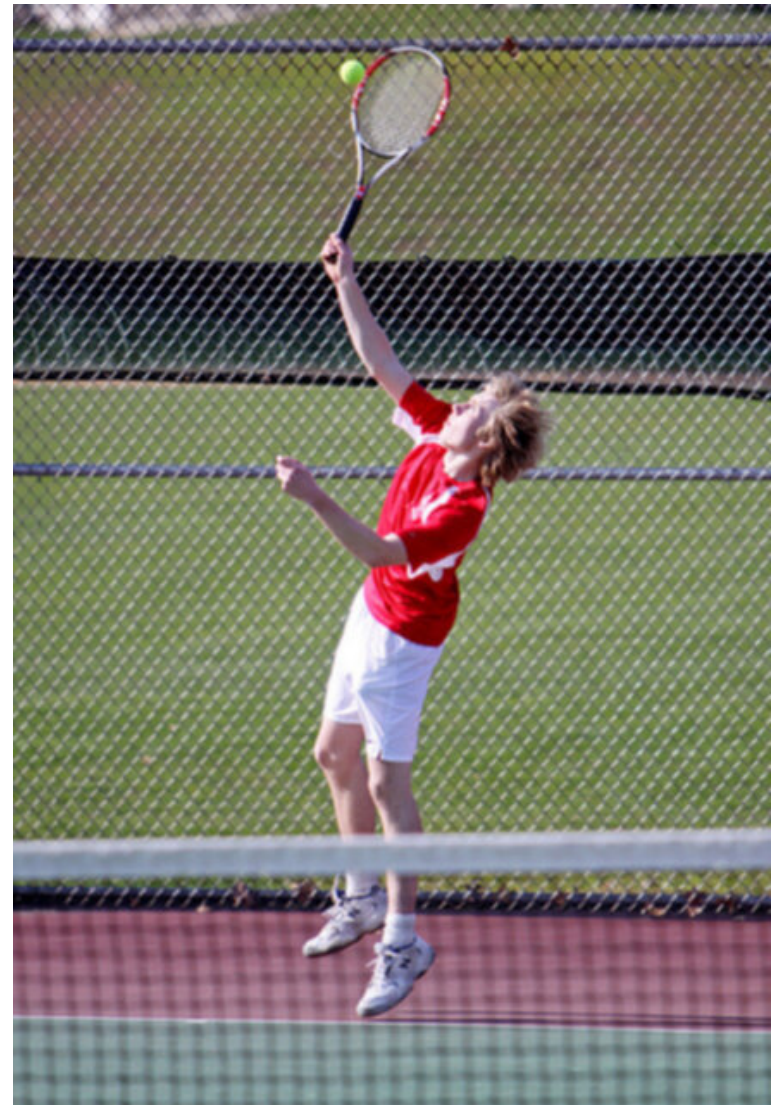

Question:is the photo in color? MRR candidate set 
1. yes

2. yes it is

3. yes, it is in color

4. yes it is in color

5. yes it 's in color

Top 10 from the remaining NDCG candidates

1. yes, it is

2. yes the picture is in color

3. yeah, this photo is in color

4. yes in color

5. it is

6. it is in color

7. yup

8. it is a beautiful color picture

9. ye

10. $\mathrm{y}$

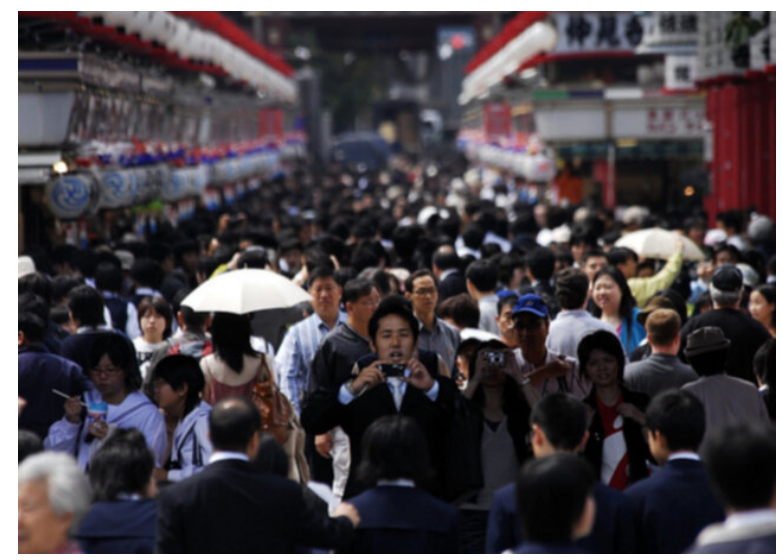

Question:any buildings visible?

MRR candidate set

1. yes a few

2. yes

3. yes, a few buildings

4. yess

5. i think so

Top 10 from the remaining NDCG candidates

1. yeah in the distance
2. lots

3. in the distance

4. yes there are some small ones

5. yes, 1 across the street

6. yes in the far background

7. yes, but i ca n't tell what kind

8. yes, it is

9. very far in the background

10. no

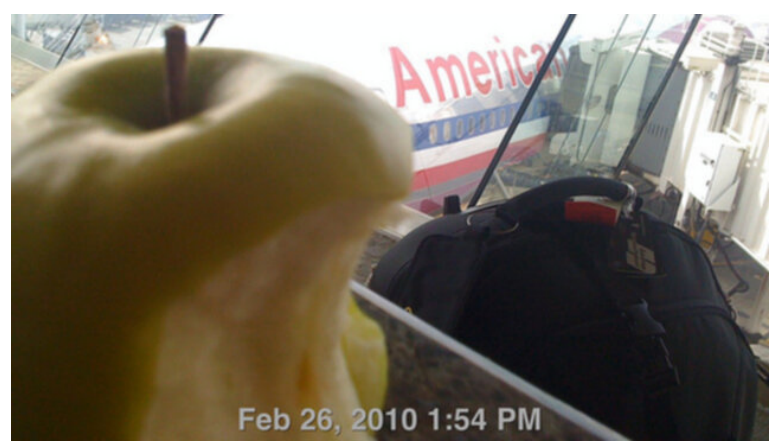

Question:is there anything made out of plastic ? MRR candidate set

1. no

2. yes

3. nope

4. not that $i$ can see

5. i do n't think so

Top 10 from the remaining NDCG candidates

1. ca n't tell

2. no that i can see

3. not sure

4. ca n't see

5. i ca n't tell

6. i can not tell

7. maybe

8. i think so

9. not really

10. yes, looks like a box 


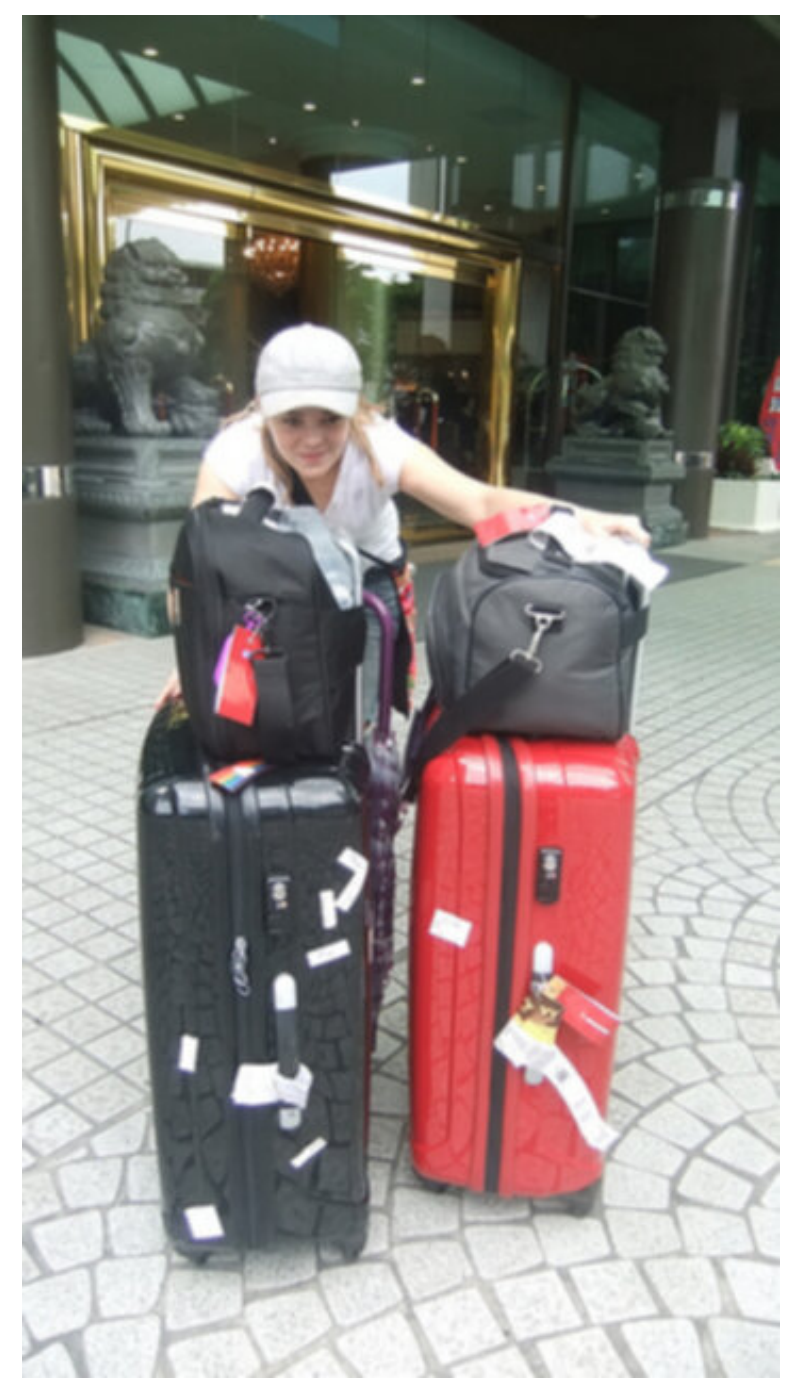

Question:how old is the woman?

\section{MRR candidate set}

1. she looks like in her 30 's

2. in her twenties i believe

3. not sure

\section{Top 10 from the remaining NDCG candidates}

1. 20 's

2. early 20 's

3. i ca n't tell

4. ca n't tell

5. 20 's maybe

6. looks to be late $20 \mathrm{~s}$

7. i can not tell

8. young adult

9. 25
10. early 30 s maybe

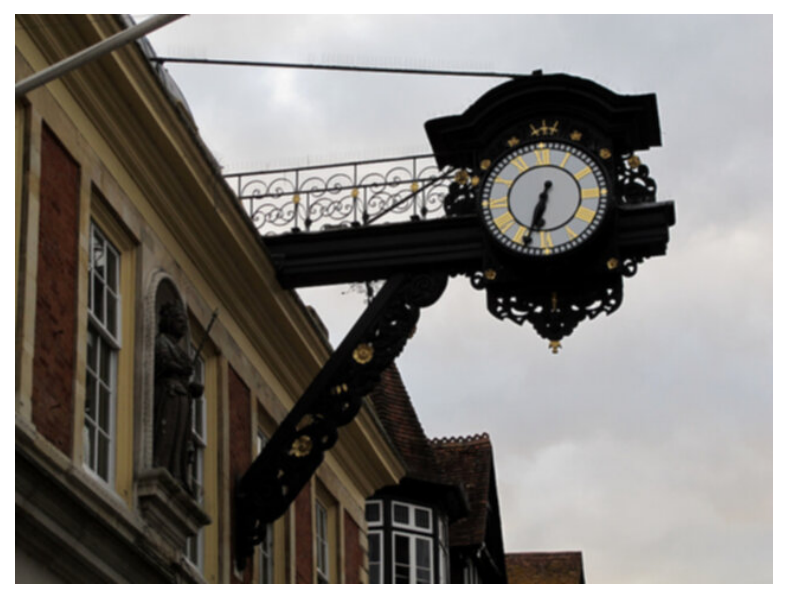

Question:is the image in color? MRR candidate set

1. yes

2. yes it is

3. yes, it is

4. yes, it is in color

5. yes it is in color

\section{Top 10 from the remaining NDCG candidates}

1. yes this is a color image

2. yes, it 's in color

3. it is yes

4. yes this picture is in color

5. yes the photo is in color

6. yes in color

7. yep

8. it is in color

9. yes it 's color

10. ye 


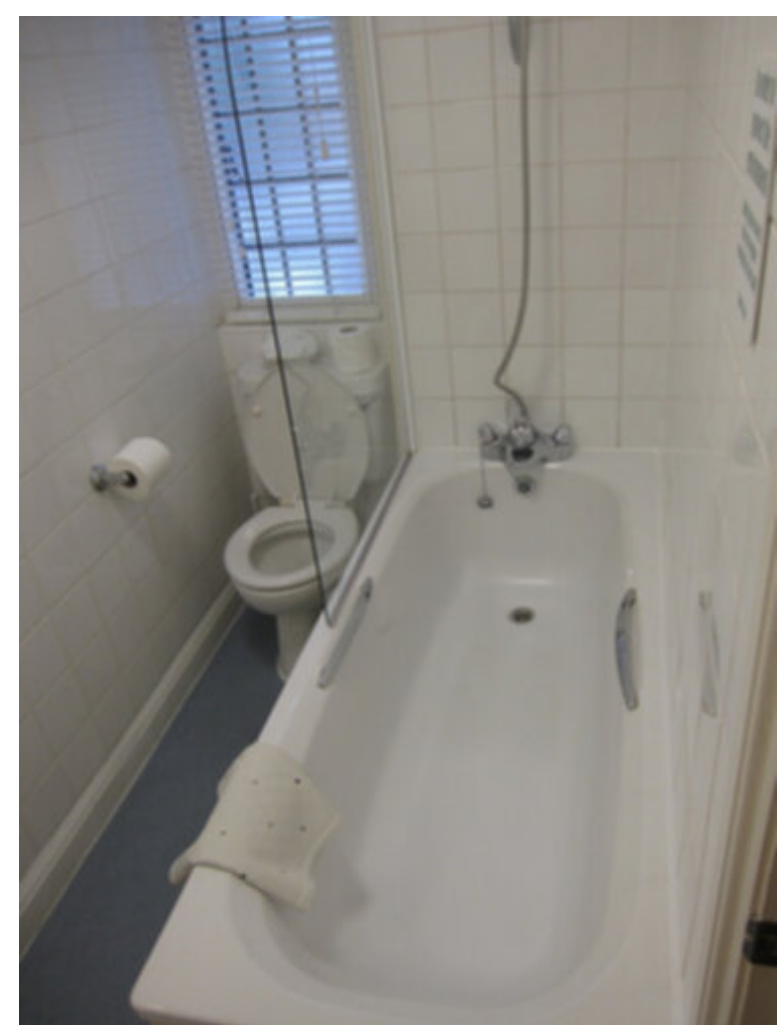

Question:is anyone in the bathroom?

\section{MRR candidate set}

1. no

2. no the bathroom

3. not that i can see

4. no people

5. nope

6. i do n't think so

\section{Top 10 from the remaining NDCG candidates}

1. no it appears empty

2. not it 's not visible

3. no he 's not

4. i would say no

5. no, there is no 1 in the kitchen

6. UNK

7. don' t know

8. 0

9. not really

10. no people this is the sky

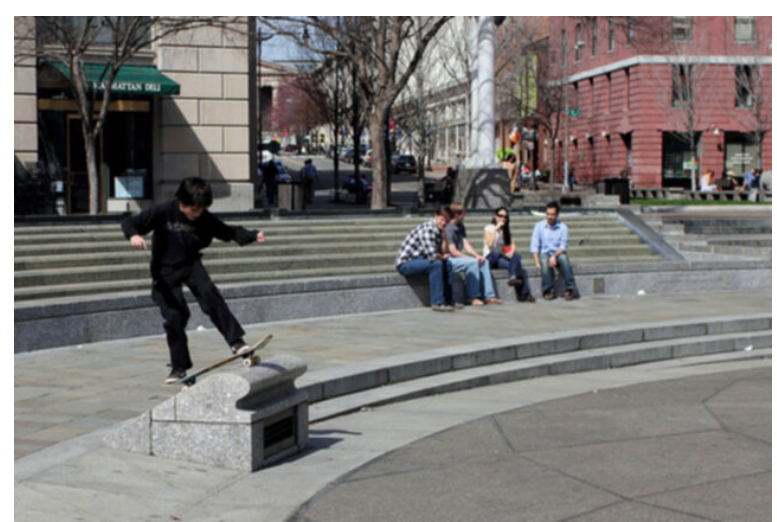

Question:is he wearing a hat?

MRR candidate set

1. no

2. no he is not

3. nope

4. i do n't see 1

Top 10 from the remaining NDCG candidates

1. i do n't think so

2. no, no hats

3. no baseball hat

4. not that i can see

5. no ca n't see it

6. no he is not wearing a helmet

7. ca n't tell

8. i ca n't tell

9. can not tell

10. not really

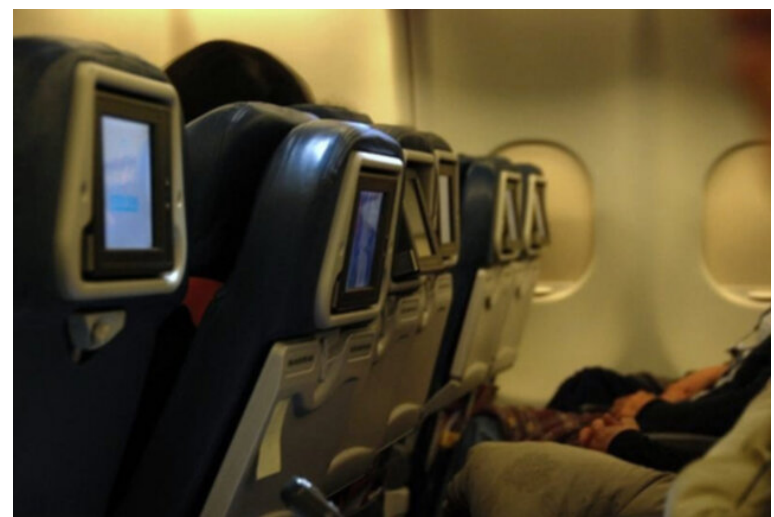

Question:are there any people ?

MRR candidate set 
1. no

2. yes

3. not that i can see

4. i do n't see any

5. nope

\section{Top 10 from the remaining NDCG candidates}

1. i ca n't see any, no

2. no people

3. no people are visible

4. no, there are n't any people in the image

5. no there are n't

6. no people in the photo

7. 0 i can see

8. not that you can see

9. i ca n't see

10. on the screens there are

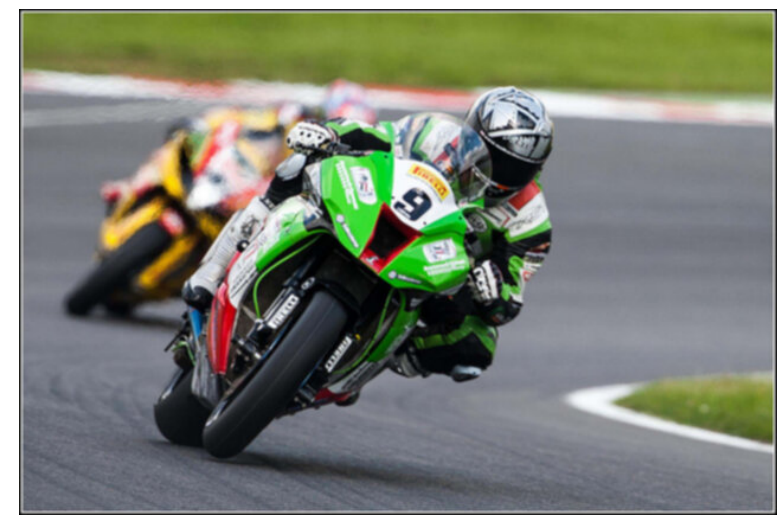

Question:do they have shoes on ?

\section{MRR candidate set}

1. yes

\section{2. i can not tell}

3. i see 1 shoe the other person is to blurry to tell

4. it looks like it

5. it 's hard to tell

6. i ca n't tell

Top 10 from the remaining NDCG candidates
1. looks like it

2. i guess so, it is hard to tell

3. yes i think so, you ca n't really see

4. ca n't tell

5. i think so

6. they are

7. not sure

8. no, they do not

9. ca n't see

10. yes they are green

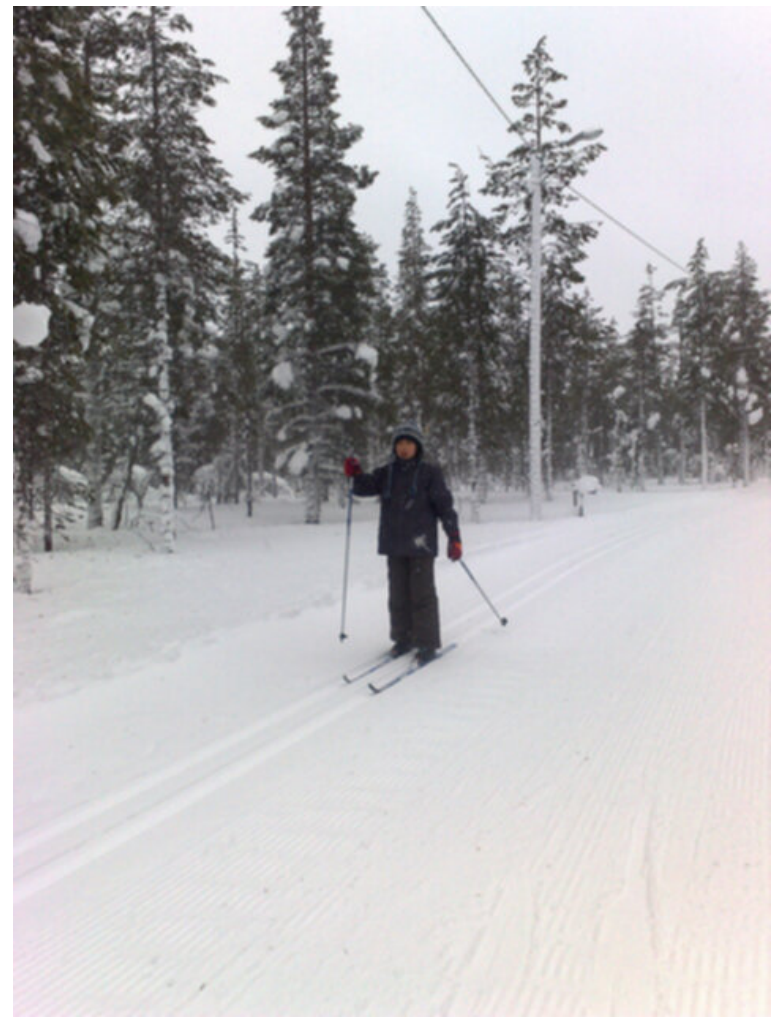

Question:does it look like a ski resort ? MRR candidate set

1. no

2. i can not tell

3. i do n't think so

4. no it does n't

5. probably not

6. nope

Top 10 from the remaining NDCG candidates 
1. not really

2. i ca n't tell

3. no i ca n't

4. i do n't think so but it 's hard to tell

5. not that i can see

6. not sure

7. no, there is n't

8. maybe, i ca n't really tell

9. possibly

10. not in the picture

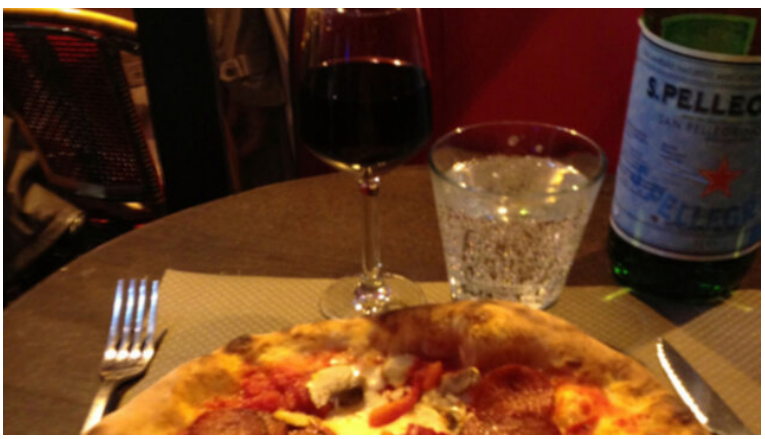

Question:is the picture in color?

MRR candidate set

1. yes

2. yes it is

3. yes, it is

4. yes it is in color

5. yes it is a color image

\section{Top 10 from the remaining NDCG candidates}

1. yeah, this photo is in color

2. yes in color

3. yes, it is fully colored

4. yyes

5. full blown color

6. i think so

7. looks like it

8. it is , but there is not much color in the photo due to the objects
9. somewhat, yes

10. looks it

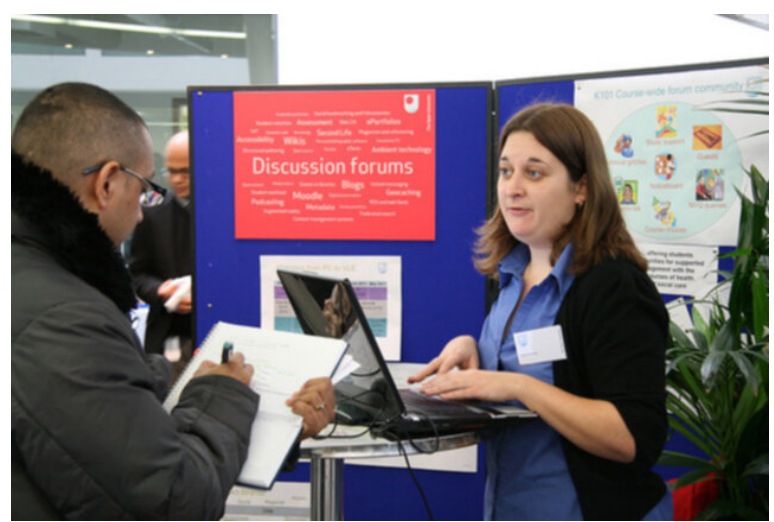

Question:is she wearing a blazer ?

MRR candidate set

1. no

2. no, sweater

3. nope

4. not that i can tell

5. i do n't think so

Top 10 from the remaining NDCG candidates

1. no a sweater

2. not that $\mathrm{i}$ can see

3. yes, a black 1

4. a long sleeve blouse

5. not sure

6. yes

7. can not see 1

8. do n't know

9. 0 seen

10. not really 


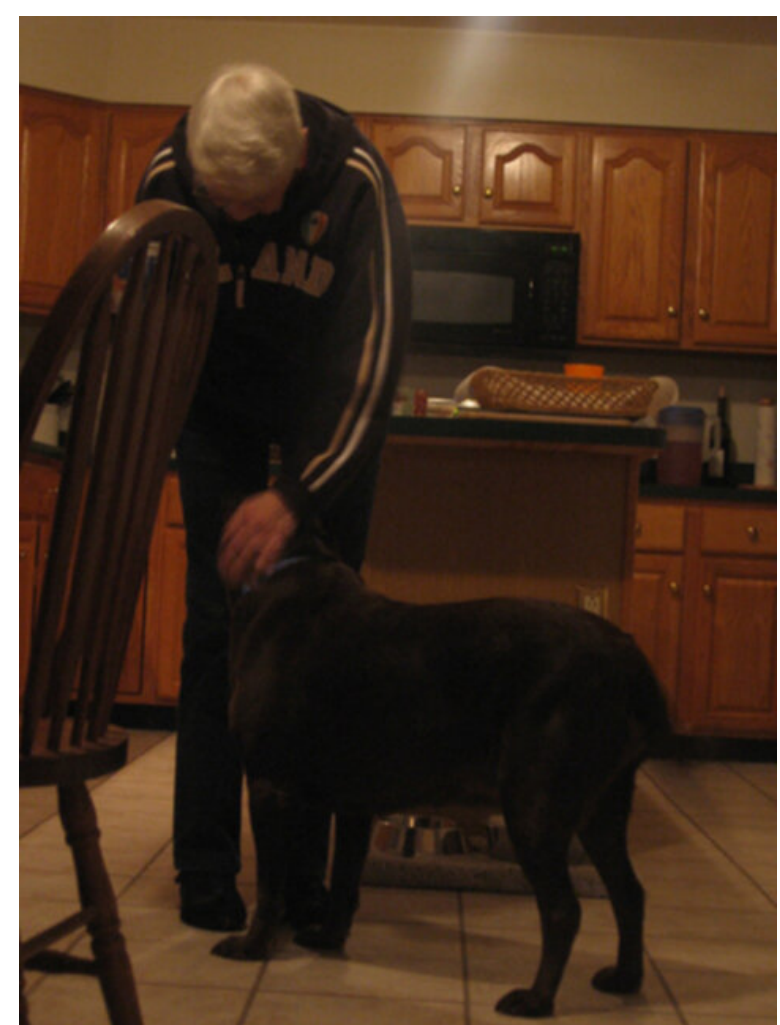

Question:does he look angry or otherwise UNK ? MRR candidate set

1. no

2. ca n't tell

3. you ca n't really see his face

4. i ca n't tell

\section{Top 10 from the remaining NDCG candidates}

1. i ca n't see that

2. i can not tell

3. not real sure

4. nope

5. not really

6. not sure

7. i do n't think so

8. looks to be sleeping if anything

9. not that i can see

10. it looks determined, but not angry

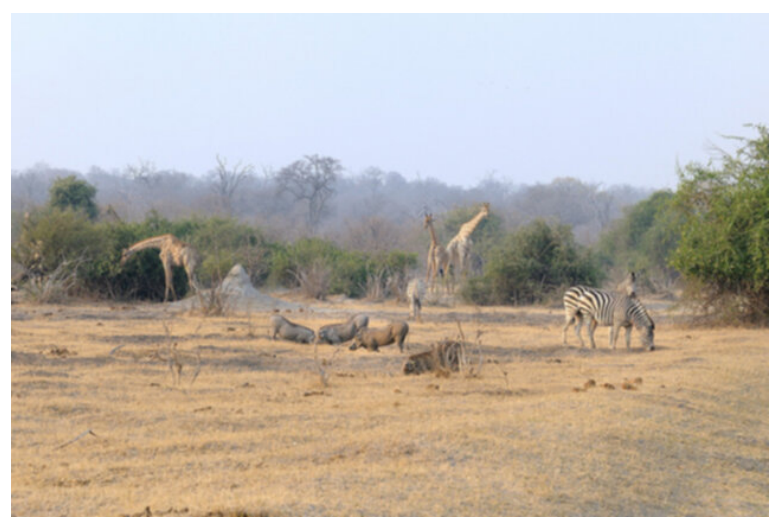

Question:are they UNK giraffes or UNK ? MRR candidate set

1. i can not tell

2. there are both

3. i ca n't tell

4. not sure

5. ca n't tell

Top 10 from the remaining NDCG candidates

1. some of each

2. i do n't think so

3. hard to tell, but i do n't think so

4. it looks to be that way

5. no

6. they are all various sizes

7. i think so

8. both

9. ca n't tell too close up

10. there are 2 UNK ones 


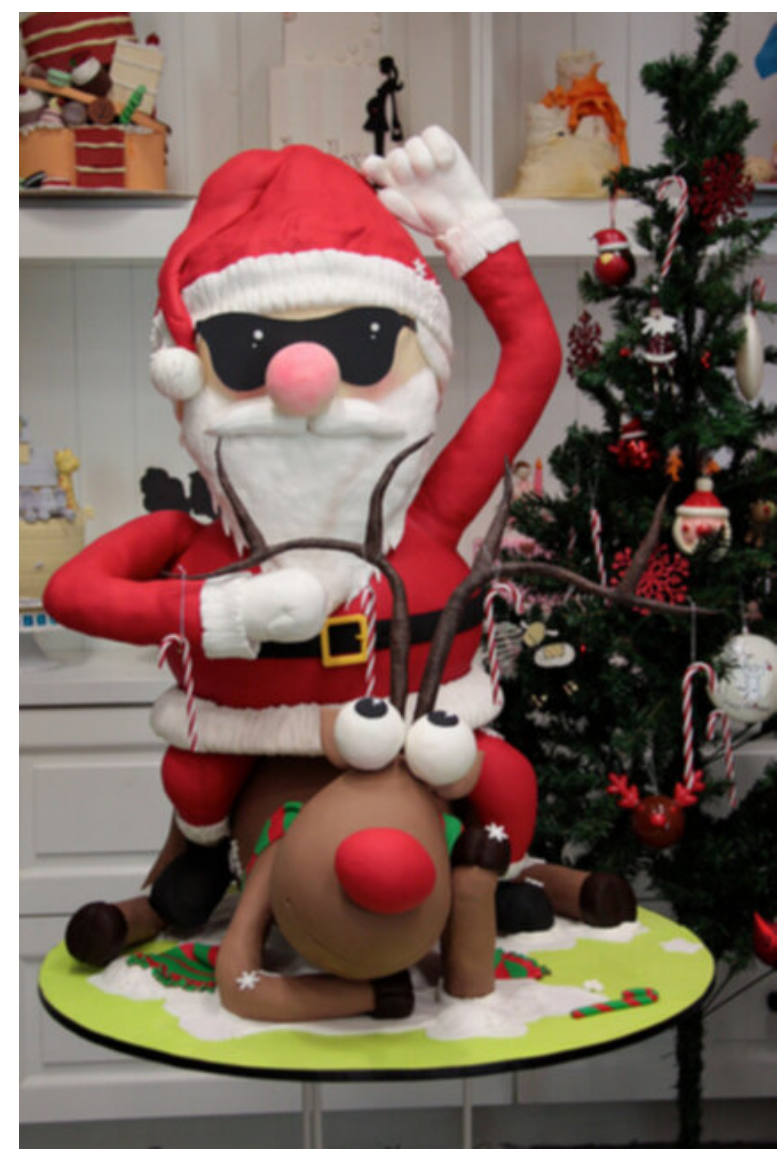

Question:what color is the reindeer?

MRR candidate set

1. brown, with a red nose

2. red

3. brown

4. brown and white

5. mostly white

\section{Top 10 from the remaining NDCG candidates}

1. white

2. golden

3. white and tan

4. brown, i think

5. white with a red stripe

6. brown, white and black

7. dark brown

8. gold

9. it is brownish gray

10. mostly white with some black

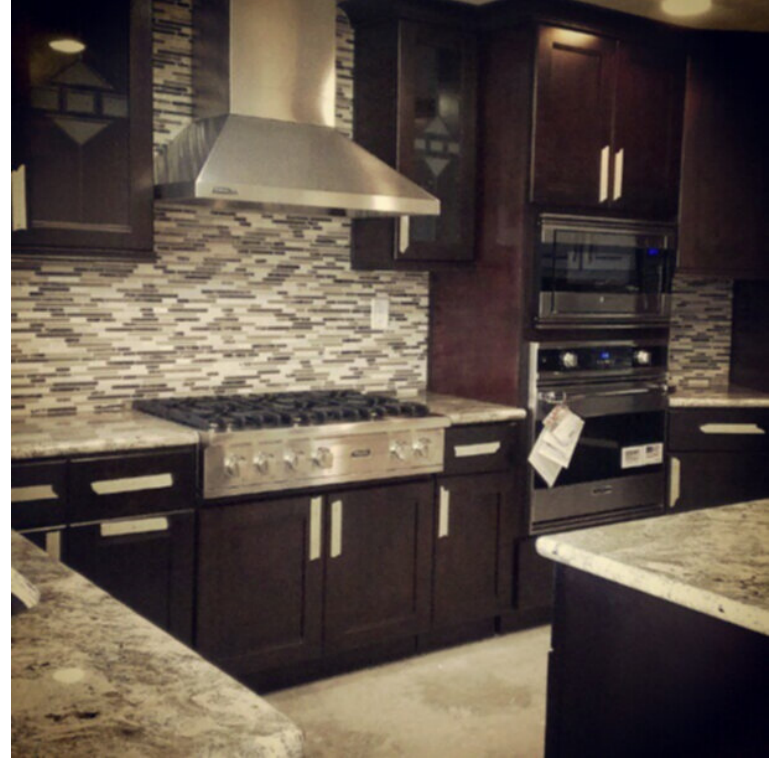

Question:can you tell what type they are, stone or laminate?

MRR candidate set

1. they are stone

2. i can not

3. i can not tell

4. i ca n't tell

5. no i'm not sure

6. no, i can not

Top 10 from the remaining NDCG candidates

1. ca n't tell

2. i can' tell, it's just white

3. nope

4. no

5. not sure

6. not really

7. no, i' $\mathrm{m}$ bad at that

8. i ca n't te

9. no, i can only see the top

10. not that i can see 


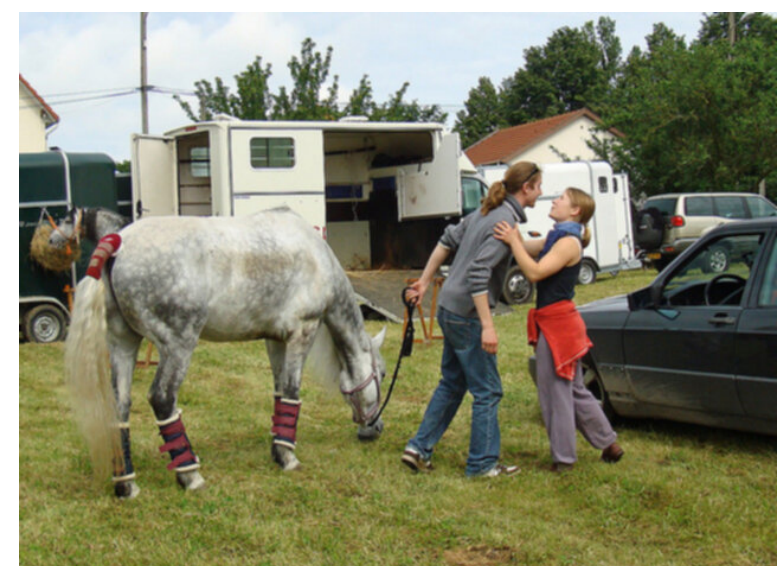

Question:any horses?

MRR candidate set

1. no

2. yes

3. yes, 1

4. 1

5. nope

Top 10 from the remaining NDCG candidates

1. 2

2. i just see 2

3. yes, it looks like it

4. not that i can see

5. ye, 1 if not more

6. not that i see

7. i think so

8. no there is n't

9. not visible

10. barely part of 1

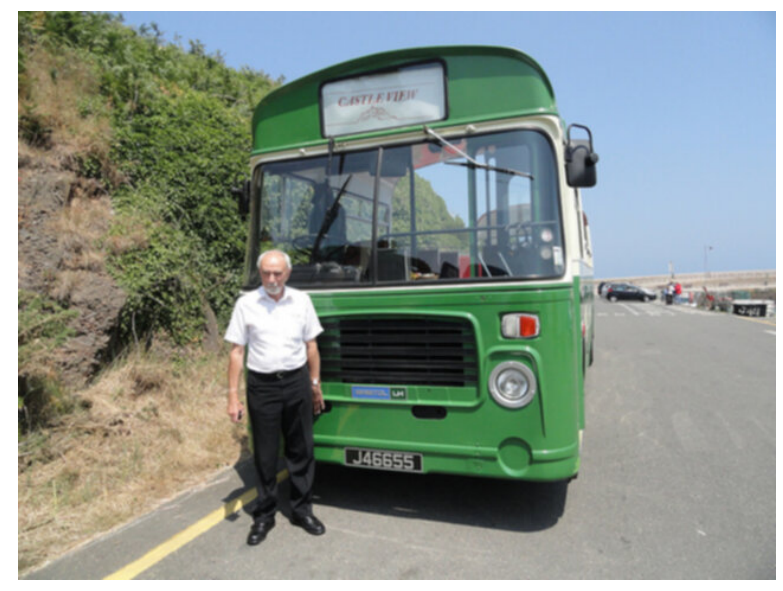

Question:are they male?

MRR candidate set

1. yes

2. no

3. yes they are

4. i think so

5. i believe so

6. it seems like it

Top 10 from the remaining NDCG candidates

1. 1 is

2. yes 1 is

3. hard to tell

4. yes!

5. i ca n't tell

6. ca n't tell

7. i can not tell

8. not sure

9. no idea not visible

10. ca n't tell they are far away in background

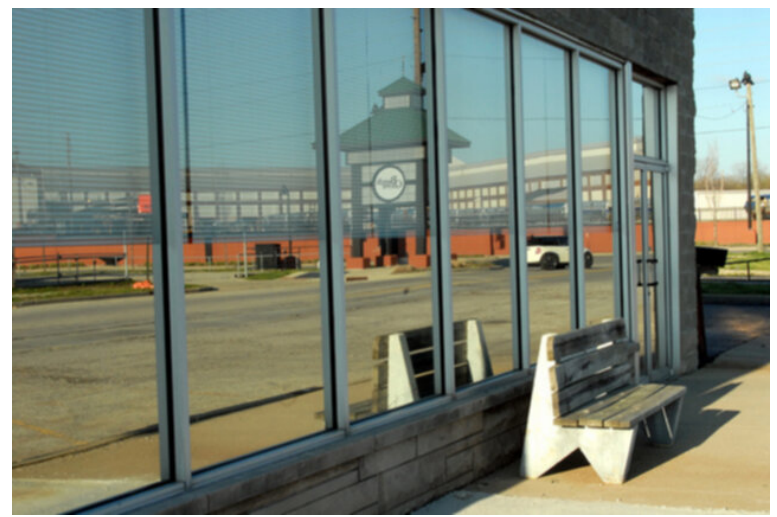

Question:is the bench in front of a building ? MRR candidate set

1. yes, it is

2. yes

3. yes in front of glass

4. no it is n't

5. nope 


\section{Top 10 from the remaining NDCG candidates}

1. no

2. yes it is

3. yes looks like it

4. not that i can see

5. looks like it

6. appears so

7. i think so

8. i see a building on the left side, but only a bit of

9. i can not tell

10. i think so, it 's hard to tell

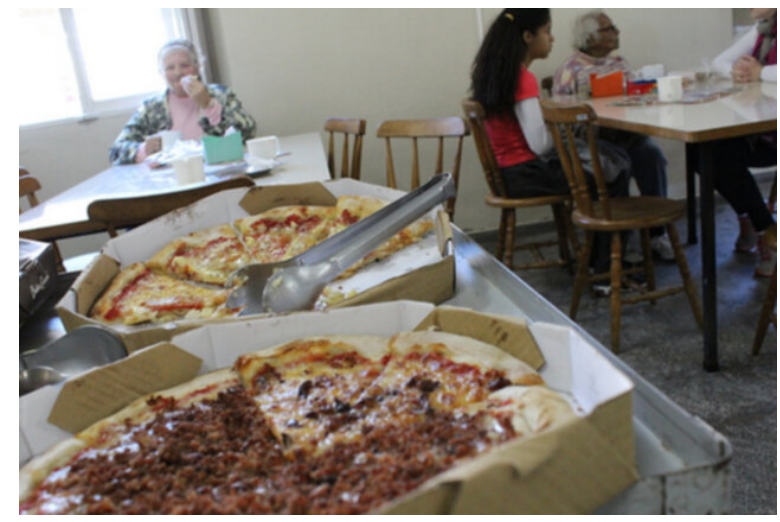

Question:how many pizzas?

\section{MRR candidate set}

1. i can see 2 kinds of pizzas

2. 2

3. there are 3

4. there are 2

5. i see 2

Top 10 from the remaining NDCG candidates

1. 2 in the scene

2. 3

3. i think 4

4. just 1

5. i can not tell

6. 4
7. it looks like a box of them and 2 sitting out

8. they are like 4

9. 1

10. i ca n't tell

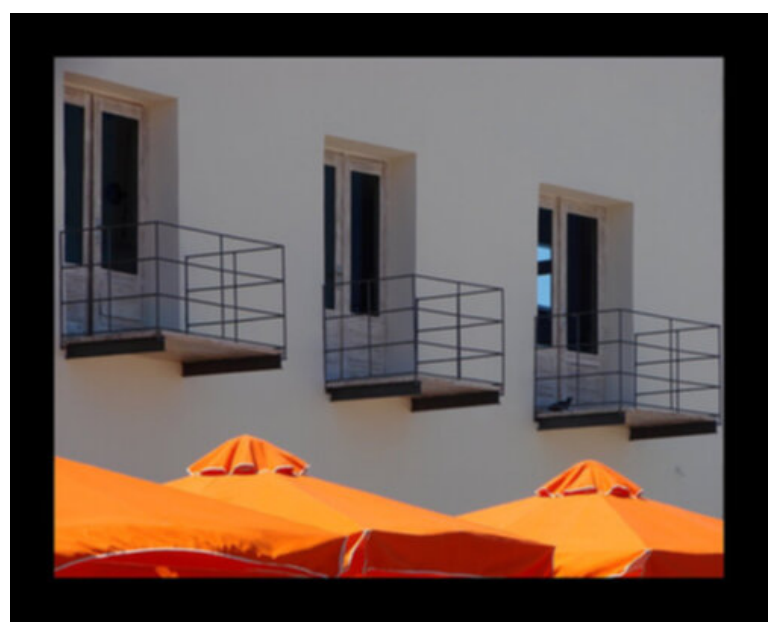

Question:what color is the building ?

MRR candidate set

1. white

2. grey

3. it is white

4. greyish

5. beige white

Top 10 from the remaining NDCG candidates

1. white and black

2. blue

3. beige

4. blue with a glass door

5. bright blue

6. off white with some UNK of gray and red brown throughout

7. there is 1 building that is a cream color

8. it is glass

9. crème

10. it 's a brownish white color 


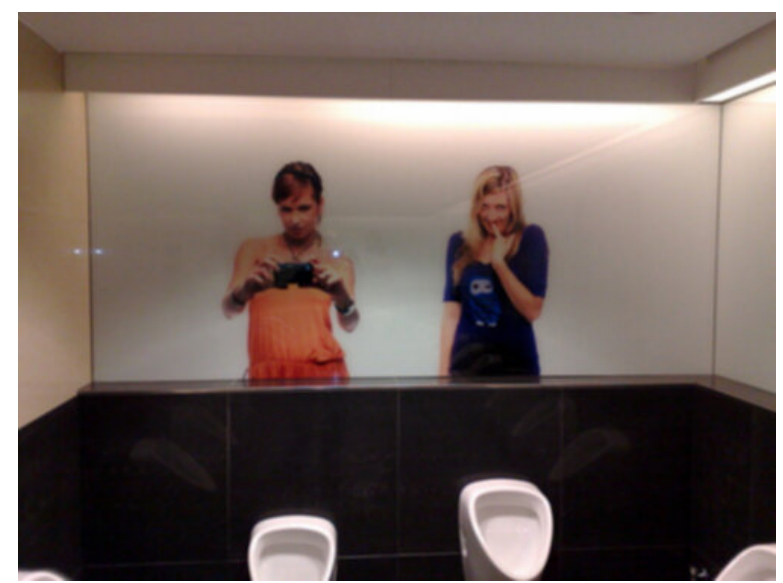

Question:is the photo in color?

\section{MRR candidate set}

1. yes

2. yes it is

3. yes, it is

4. yes, it is in color

5. yes, the photo is in color

6. yes it is in color

Top 10 from the remaining NDCG candidates

1. yes, the image is in color

2. yes in color

3. yes, the picture is in color

4. the photo is in color

5. yes the picture is in color

6. it is

7. sure is

8. yes,

9. yup

10. ye

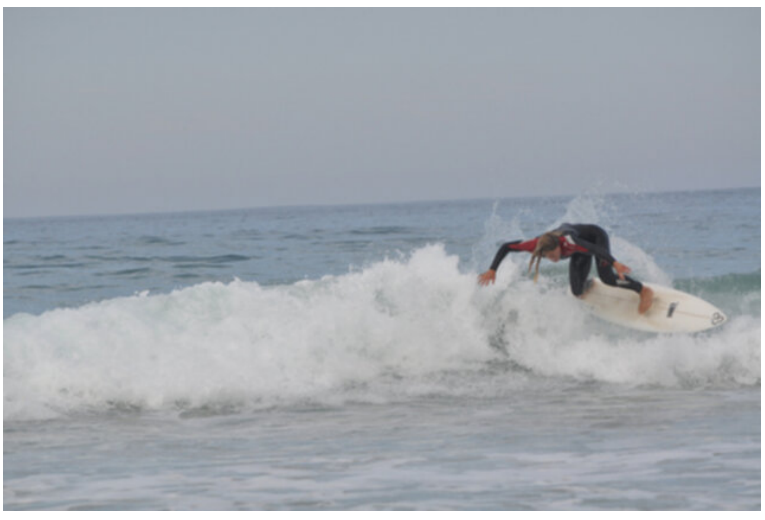

Question:is the day sunny?

MRR candidate set

1. yes

2. no

3. not really

4. nope

Top 10 from the remaining NDCG candidates

1. yes it is

2. looks like it

3. no, it is not

4. yes, it is

5. i think so

6. UNK

7. sort of

8. i do n't think so

9. i suppose

10. ca n't really see the sun think it 's pretty overcast

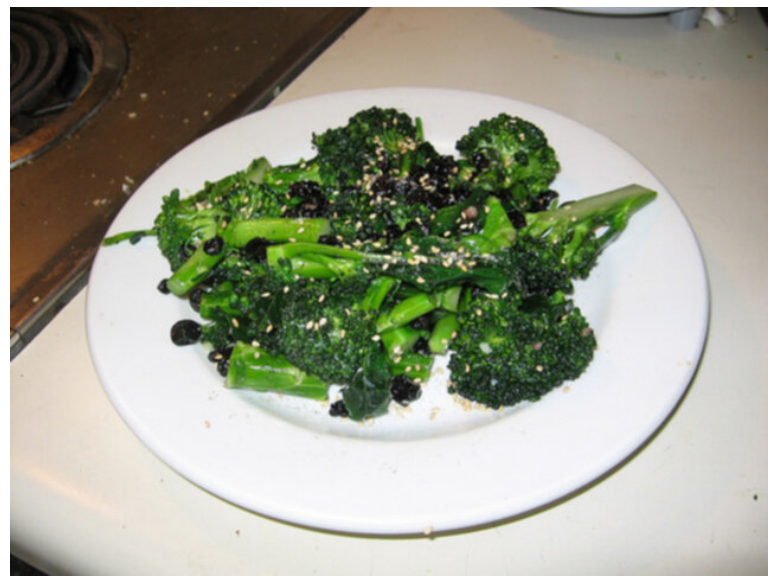

Question:are they on a white plate ? MRR candidate set

1. yes

2. yes, it is

3. yes they are

4. yes it is

5. i believe so

Top 10 from the remaining NDCG candidates 
1. it 's in color

2. yes, a brown 1

3. i think so

4. UNK

5. no it is in a paper boat

6. it's okay

7. white

8. no, just on the table

9. yes, i can see some cars

10. it is in a box

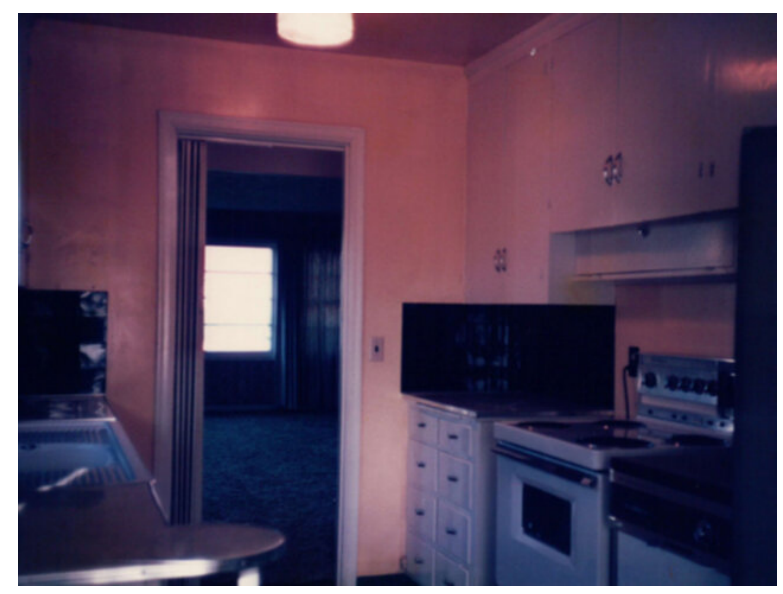

Question:is the sink made out of metal ?

MRR candidate set

1. yes

2. i can not tell

3. i ca n't tell

4. ca n't really tell

5. ca n't tell

\section{Top 10 from the remaining NDCG candidates}

1. not sure

2. no

3. yes it looks like it is

4. yes it is

5. i think so

6. looks like it

7. yes, it is
8. it look like it

9. nope

10. too far away to see that

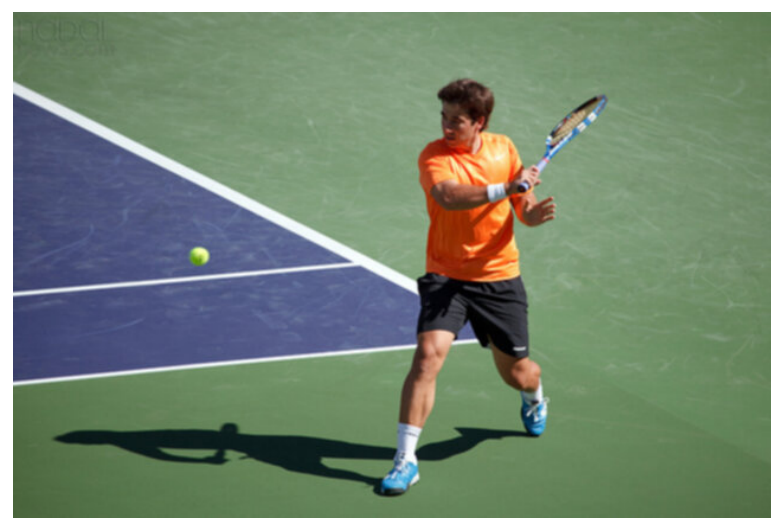

Question:is there people there apart from the man ?

\section{MRR candidate set}

1. no

2. no just 1 person

3. not that i can see

4. nope

5. i do n't see any

\section{Top 10 from the remaining NDCG candidates}

1. no other people

2. i don' $t$ see anyone at all

3. 0

4. no people are in the picture

5. i do n't think so

6. no people

7. no, there are n't any people visible

8. not really

9. no, there are n't any people

10. no people in the room 


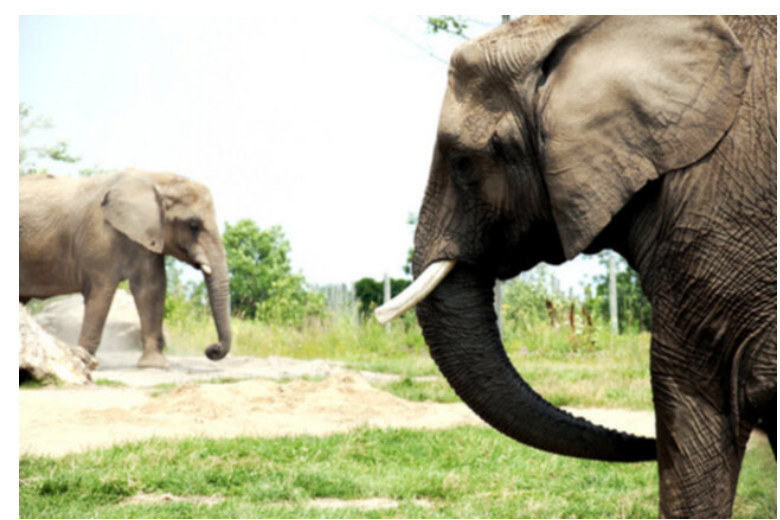

Question:are the elephants eating ?

MRR candidate set

1. no

2. yes

3. nope

4. no, do n't think so

Top 10 from the remaining NDCG candidates

1. no does n't look like it

2. i do n't think so

3. not that i can see

4. no standing

5. it looks like it

6. only the 1 with its head down is

7. i think so

8. yes they appear to be

9. yes , they appear to be

10. 1 of the 2 of them are

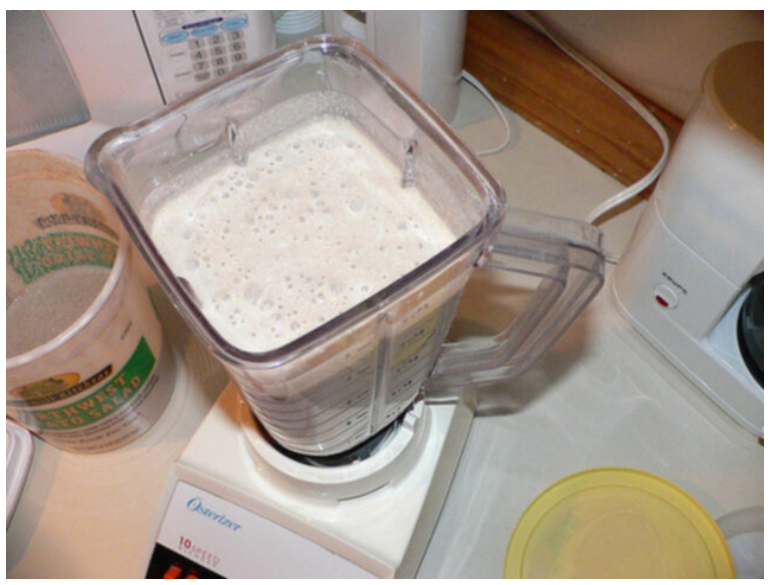

Question:is it a cold drink?

MRR candidate set
1. no

2. yes

3. no it isn

4. nope

Top 10 from the remaining NDCG candidates

1. looks that way

2. i do n't think so

3. no not really

4. i can not tell

5. looks like it

6. looks like it is

7. no sure

8. yes it is

9. looks like

10. it appears to be

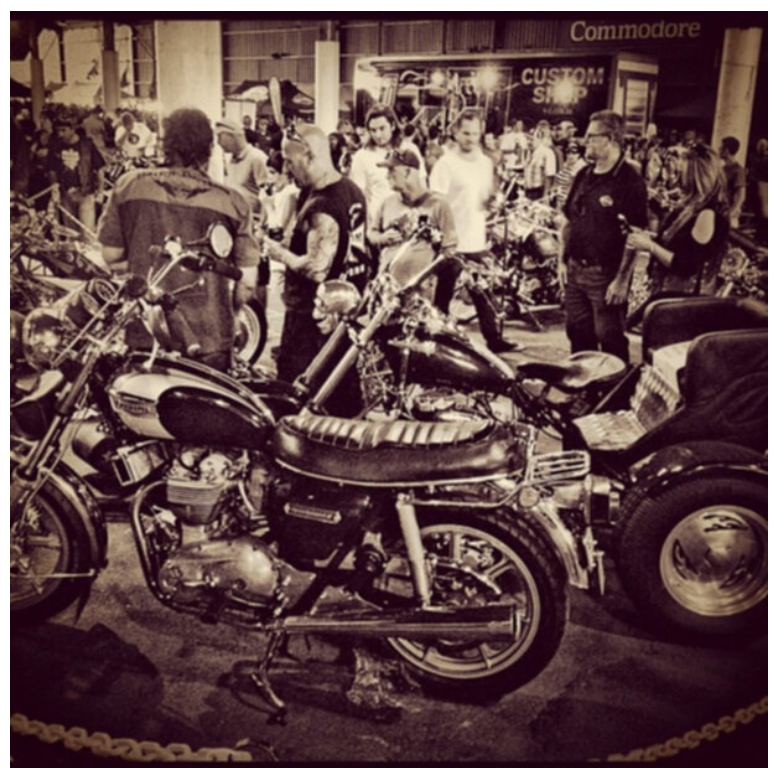

Question:do they look scary?

MRR candidate set

1. no

2. not really

3. yes kind of

4. yes

5. not particularly 
6. nope

Top 10 from the remaining NDCG candidates

1. not sure

2. i do n't think so

3. not that i can see

4. i can not tell

5. hard to say

6. maybe

7. i ca n't tell

8. ca n't tell

9. i ca n't see any

10. they look serious

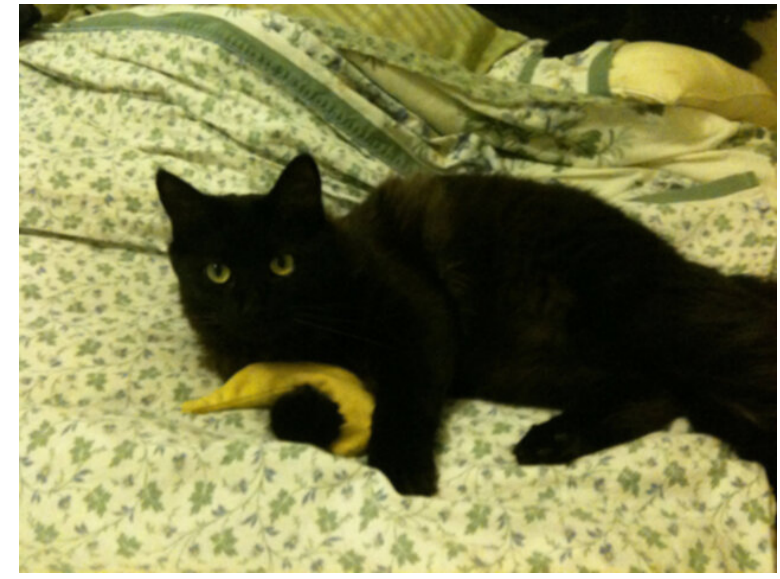

Question:are there any other animals in the picture ?

\section{MRR candidate set}

1. no

2. 0

3. nope

4. 0 at all

5. not that i can see

Top 10 from the remaining NDCG candidates

1. no, there are 0

2. i ca n't see any

3. no, just the 1

4. i do n't think so
5. nope, just 1

6. 0 sen

7. no animals

8. no animals either

9. no other people

10. no people

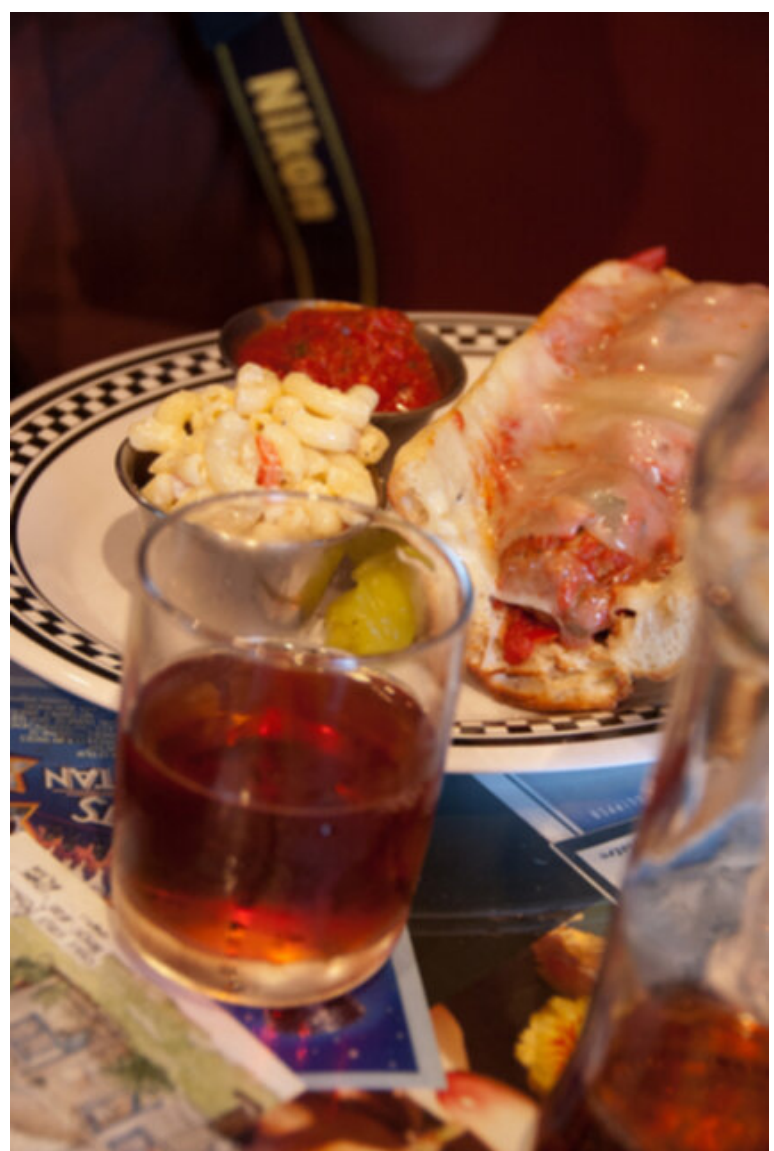

Question:does it look delicious ?

MRR candidate set

1. yes

2. yes it does

3. yes, it is

4. looks okay

5. it does yes

6. yes, making me hungry

Top 10 from the remaining NDCG candidates

1. it's okay

2. not really 
3. no not really

4. UNK

5. no it does not

6. no

7. i think so

8. no really

9. nope

10. no it's not

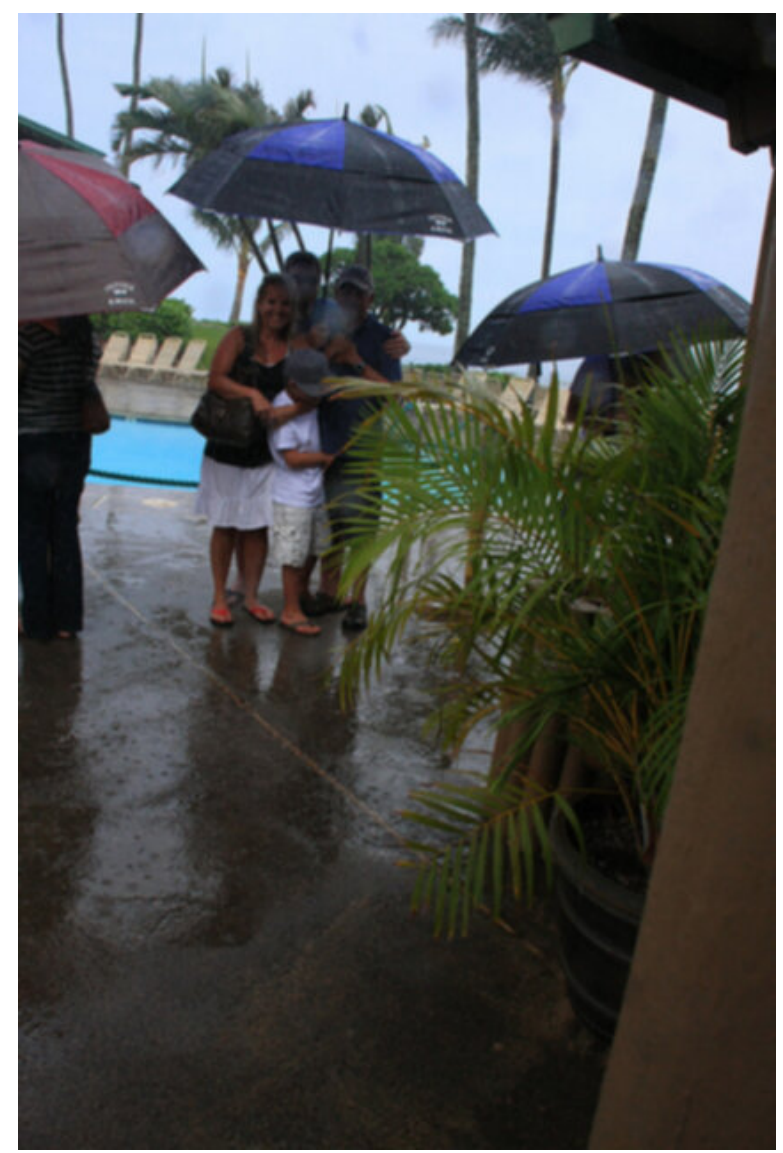

Question:are the kids playing with anything ?

MRR candidate set

1. holding umbrellas

2. no

3. yes

4. looks like a boy has something in his hand

5. nope

6. not that $\mathrm{i}$ can see

Top 10 from the remaining NDCG candidates

1. no, all are kids
2. i do n't think so

3. i can not tell

4. not really

5. yes i think so

6. nothing visible

7. yes, they are

8. i ca n't tell that

9. yes, a bunch of umbrellas

10. yes they are

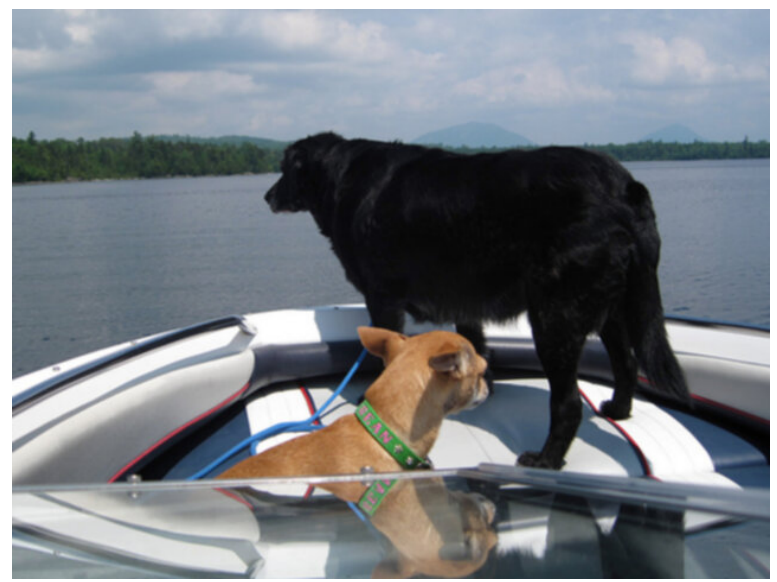

Question:are they older or younger? MRR candidate set

1. they look older

2. i think younger

3. young

4. ca n't tell

5. do n't know

6. i am not sure

7. i can not tell

8. i ca n't tell

\section{Top 10 from the remaining NDCG candidates}

1. not sure

2. looks like 1 of each

3. medium

4. they look like adults mostly

5. ca n't see their faces 
6. 1 looks old while the other looks mid 30s

7. very old

8. they look fairly big

9. no

10. i ca n't tell, but judging by the UNK, probably

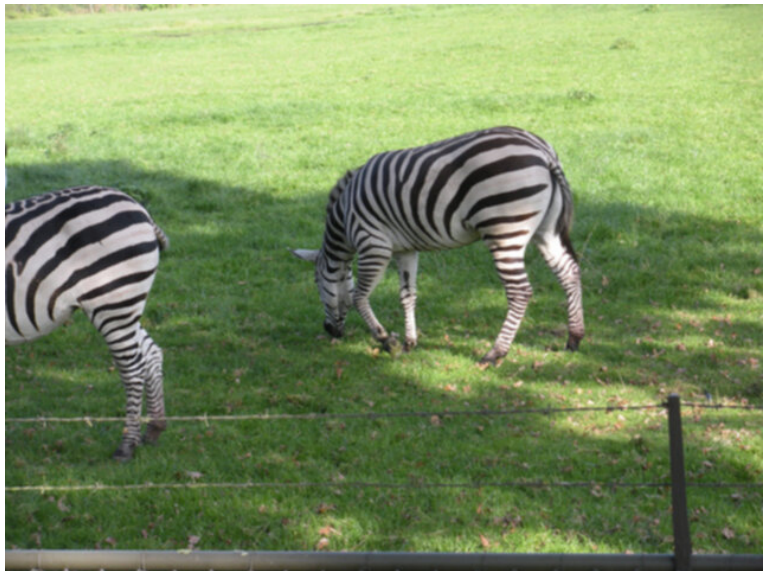

Question:is it a metal fence?

MRR candidate set

1. yes

2. wire

3. yes it is

4. it appears to be

5. yes, it is

\section{Top 10 from the remaining NDCG candidates}

1. it is

2. yes, it looks like it is

3. it looks like it

4. looks like it is

5. i think so it

6. it has some lines, but yes

7. i think so

8. yes they are

9. yes, i can see a fence

10. yes i think so, hard to tell

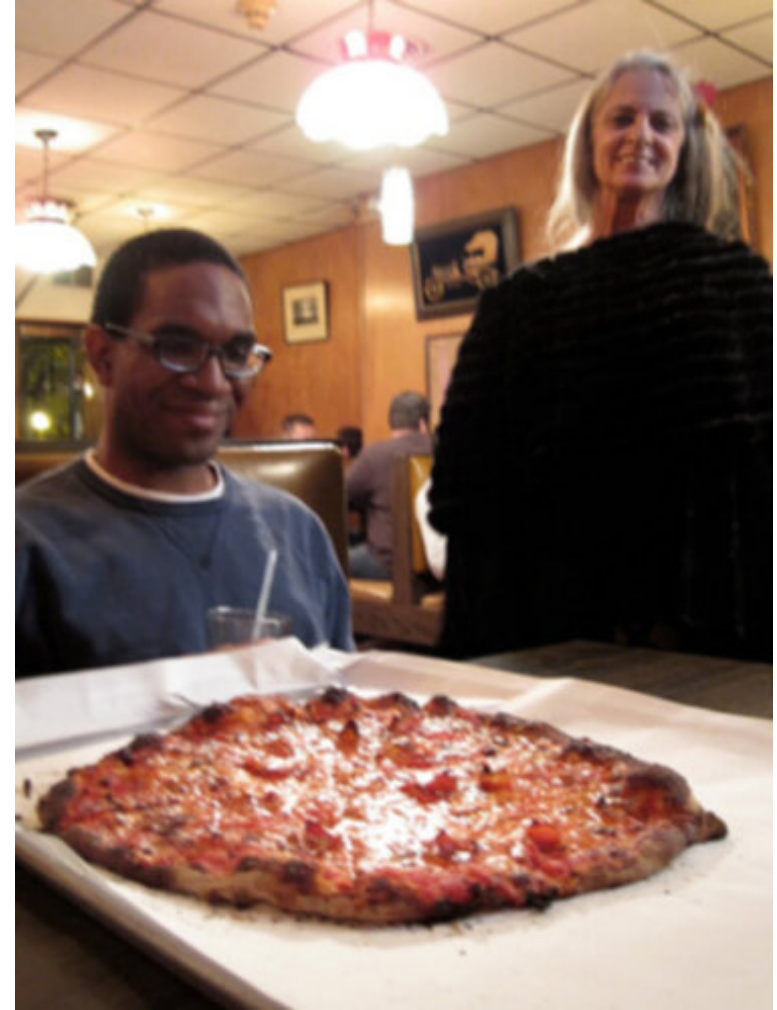

Question:are there pictures on the walls ? MRR candidate set

1. yes

2. no

3. yes there are

4. yes for sure

Top 10 from the remaining NDCG candidates

1. i think so

2. yes, 1 that $i$ can see

3. not that i can see

4. nope

5. no pictures on the wall

6. yeah, 3

7. 1

8. might be but it is hard to see

9. no, i do n't see any

10. yes, but not too many 


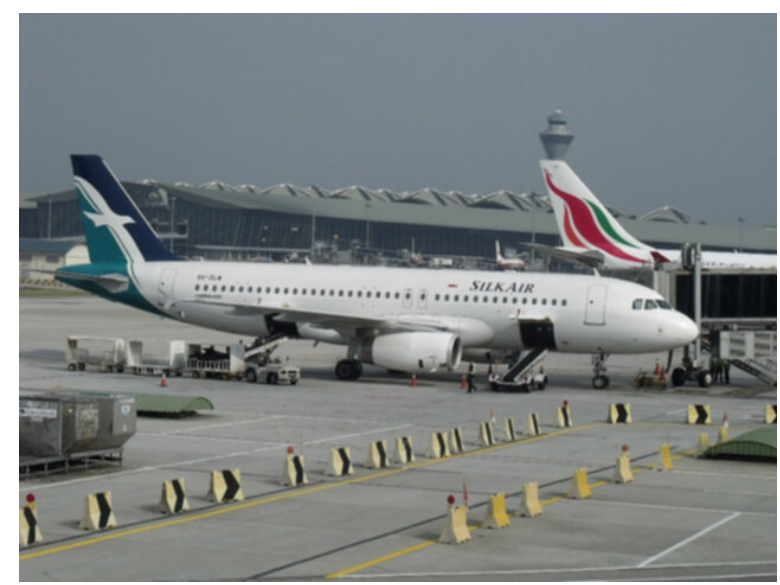

Question:can you see clouds?

MRR candidate set

1. i see no clouds the sky is fairly grey though

2. no clouds are visible

3. yes i can see the sky cloudy

4. no

5. no, it looks cloudy

Top 10 from the remaining NDCG candidates

1. no,

2. there seems to be a few clouds

3. nope

4. yes they are clouds

5. very few

6. not that i can see

7. not really

8. i do n't think so

9. yes i can

10. 0

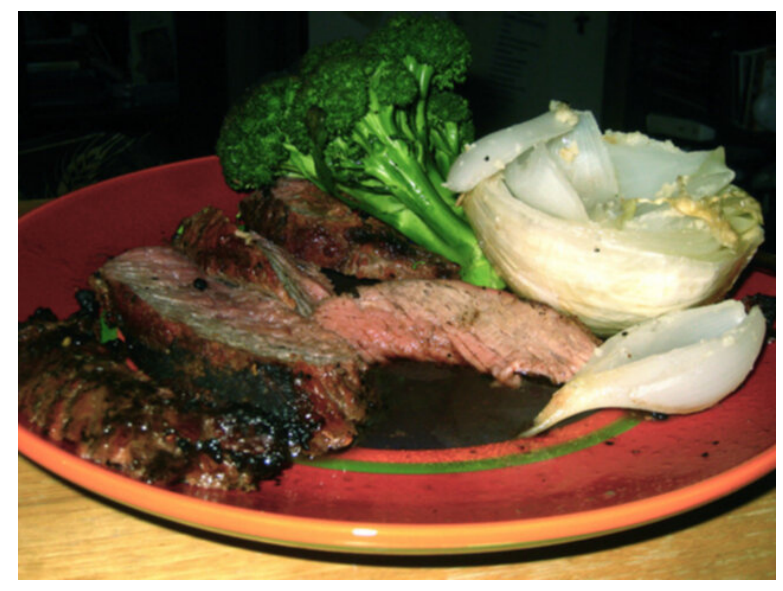

Question:where are the onions ?

MRR candidate set

1. on the right of the plate

2. you can not really tell

3. i can not tell

4. not sure

5. i ca n't tell

\section{Top 10 from the remaining NDCG candidates}

1. yes

2. ca n't tell

3. UNK

4. sitting at the table

5. it looks like a box of them and 2 sitting out

6. there is squash, shredded carrots, and shredded cabbage

7. not that i can see

8. the UNK of heaven

9. yes, it is

10. i think os

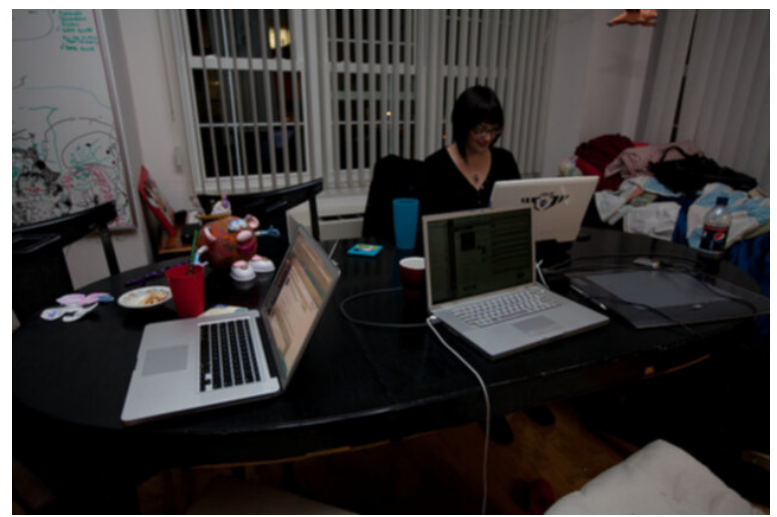

Question:does she have brown hair?

MRR candidate set

1. yes

2. yes, she does

3. yes, it is

4. i believe so

5. i think so

6. oh yeah 


\section{Top 10 from the remaining NDCG candidates}

1. black

2. yes and black

3. no

4. no, her eyes are brown

5. nope

6. it looks to be maybe of shoulder length

7. ca n't tell

8. i ca n't tell

9. yes i do

10. i can not tell

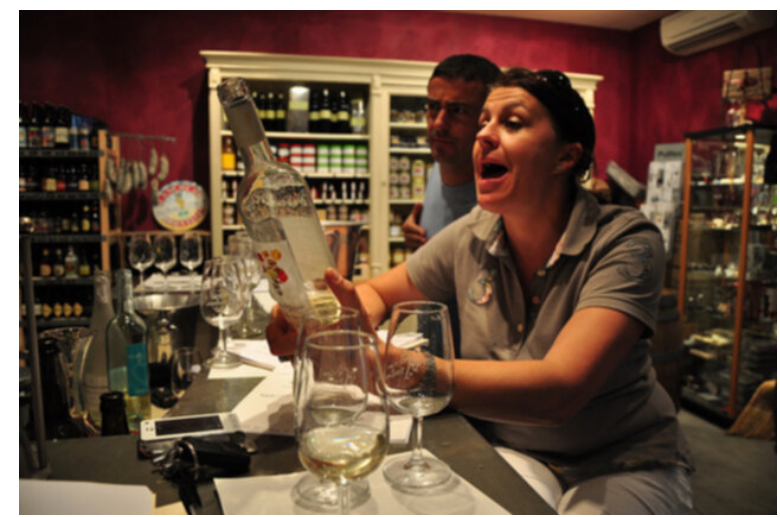

Question:are there other people?

\section{MRR candidate set}

1. there is a gentleman beside her

2. no

3. yes, there are 2 other people in the background

4. no, there is not

5. not that i can see

6. no there are not

7. nope

\section{Top 10 from the remaining NDCG candidates}

1. no other people in the photo

2. 0 that $i$ can see in the picture

3. no, there are no other people com

4. 0 i can see
5. no there 's not

6. not from what i can see

7. no, i ca n't see anyone

8. not within view

9. yes 2 people can be seen in the background

10. i do n't think so

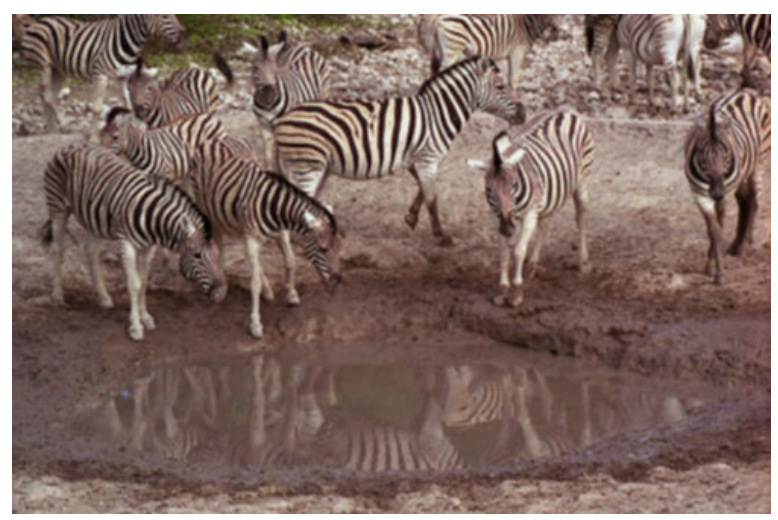

Question:how many zebras are there ?

MRR candidate set

1. 9

2. i count twelve

3. 10

4. 7

5. 8 or so

6. too many to count

Top 10 from the remaining NDCG candidates

1. about ten, of different species

2. eleven

3. at least 20

4. 11 or 12

5. looks like around a dozen

6. a lot

7. over 20

8. about $12-14$

9. about 25

10. i see 6 i think 


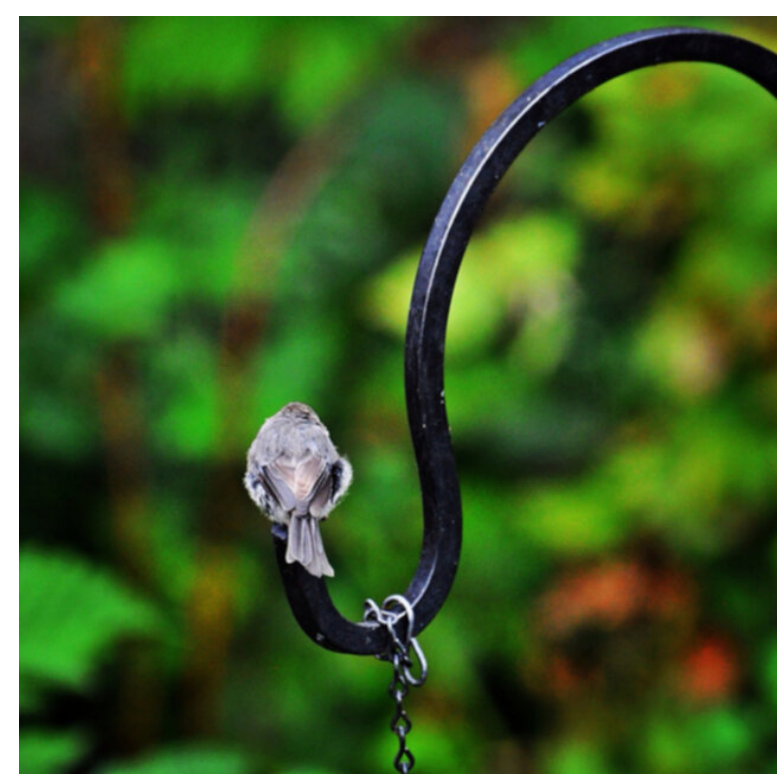

Question:is the yard large?

\section{MRR candidate set}

1. i can not tell

2. yes

3. i ca n't tell

4. ca n't tell

5. can not tell too close up

6. not sure

Top 10 from the remaining NDCG candidates

1. from what i can see

2. seems like it very close up photo

3. i think so

4. maybe

5. no

6. no, but hard to tell from the close up

7. yes it is

8. yes, it is

9. not really

10. yes it's large

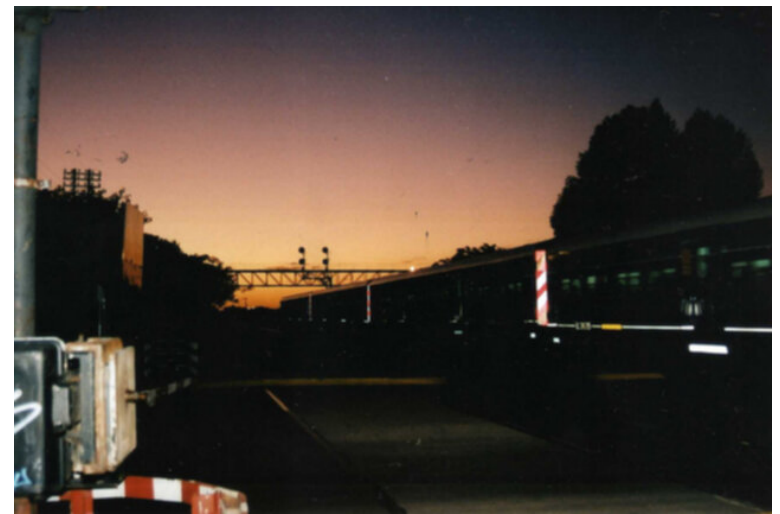

Question:does the picture match the caption? MRR candidate set

1. yes

2. no

3. yes, it does

4. yes it does match the caption

5. yes it does

6. no it is not

Top 10 from the remaining NDCG candidates

1. nope

2. no,

3. not really

4. it does

5. yes, the picture is in color

6. yes, it 's in color

7. it is

8. yes, it is

9. yes it is

10. i think so 
9. yes there are

10. 0

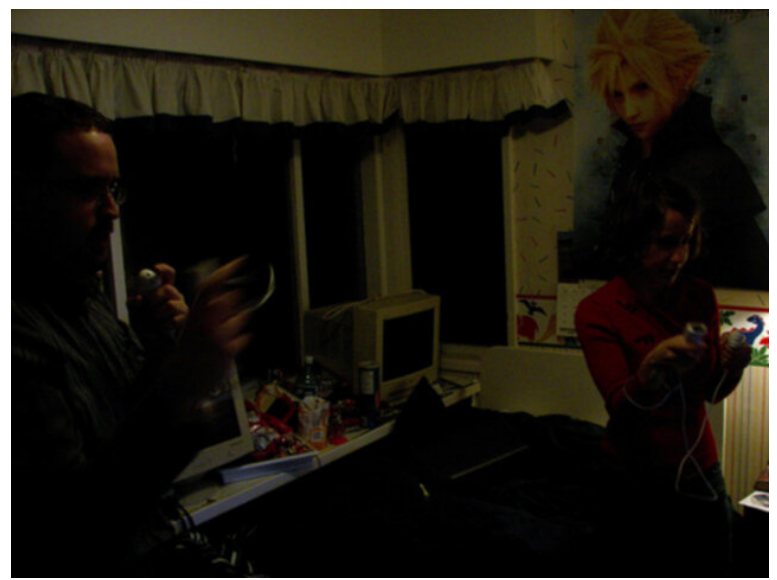

Question:how many kids are there? MRR candidate set

1. 2

2. there are 2 people but they are n't kids

3. i think 2

4. only 2

5. there is only 1

\section{Top 10 from the remaining NDCG candidates}

1. 2 , possibly 3

2. 1

3. 3

4. looks like 3

5. i can see 2 clearly and there may be part of a third but i 'm not sure

6. there is 1

7. only see 1 , too close up to see much

8. 3 total 1 looks like the guide

9. there are tow

10. i see 1 arm besides them 


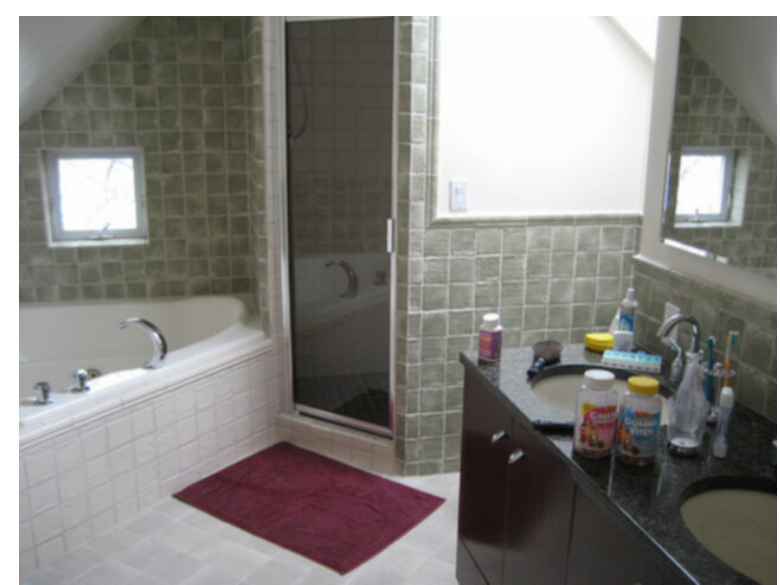

Question:are there any bath mats ?

MRR candidate set

1. no

2. yes there is a bath mat

3. i can not tell

4. nope

5. no, it does n't appear so

\section{Top 10 from the remaining NDCG candidates}

1. yes

2. yes there are

3. no,

4. yes there is 1

5. there are

6. not that i can see

7. not that you can see

8. just 1

9. i do n't think so

10. 0 that $i$ can see

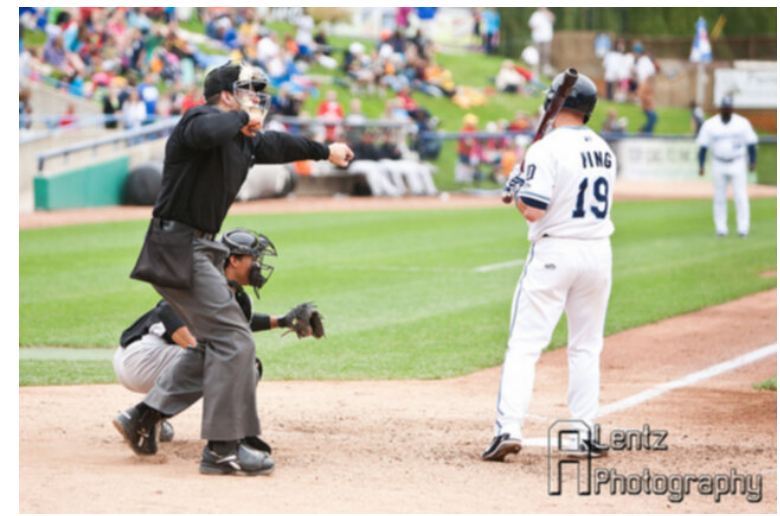

Question:can you see the sky?

MRR candidate set
1. no

2. no i can not

3. no i ca n’t

4. no, i can not

5. nope

Top 10 from the remaining NDCG candidates

1. no you ca n't

2. no i ca n't see the sky

3. no i do not

4. no, the sky is not visible

5. not really

6. no i can UNK

7. just a little bit

8. $\mathrm{n}$

9. i do n't think so

10. a little bit

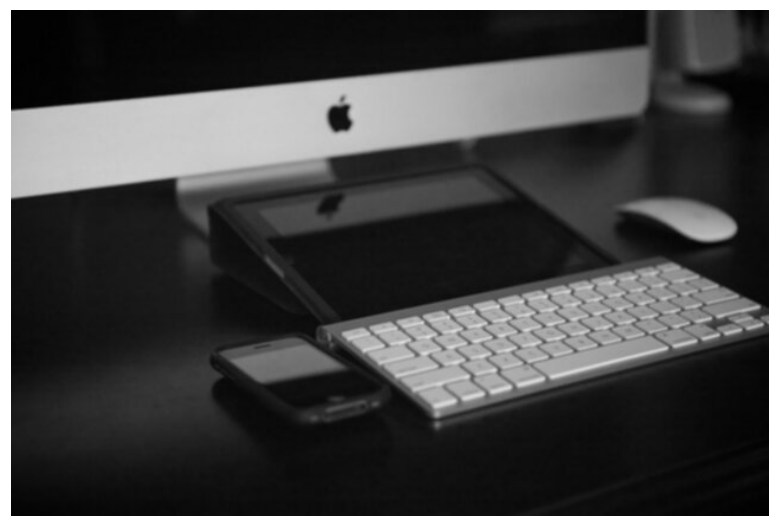

Question:is there a chair?

MRR candidate set

1. no, i can only see the close up of what' $\mathrm{s}$ on the desk

2. no

3. not that i can see

4. i do n't see 1

5. no there is n't

\section{Top 10 from the remaining NDCG candidates}

1. not that can be seen 
2. not visible

3. nope

4. do not see 1

5. do n't see any

6. 0 that i can see

7. ca n't tell

8. i do n't think so

9. i can not tell

10. not sure

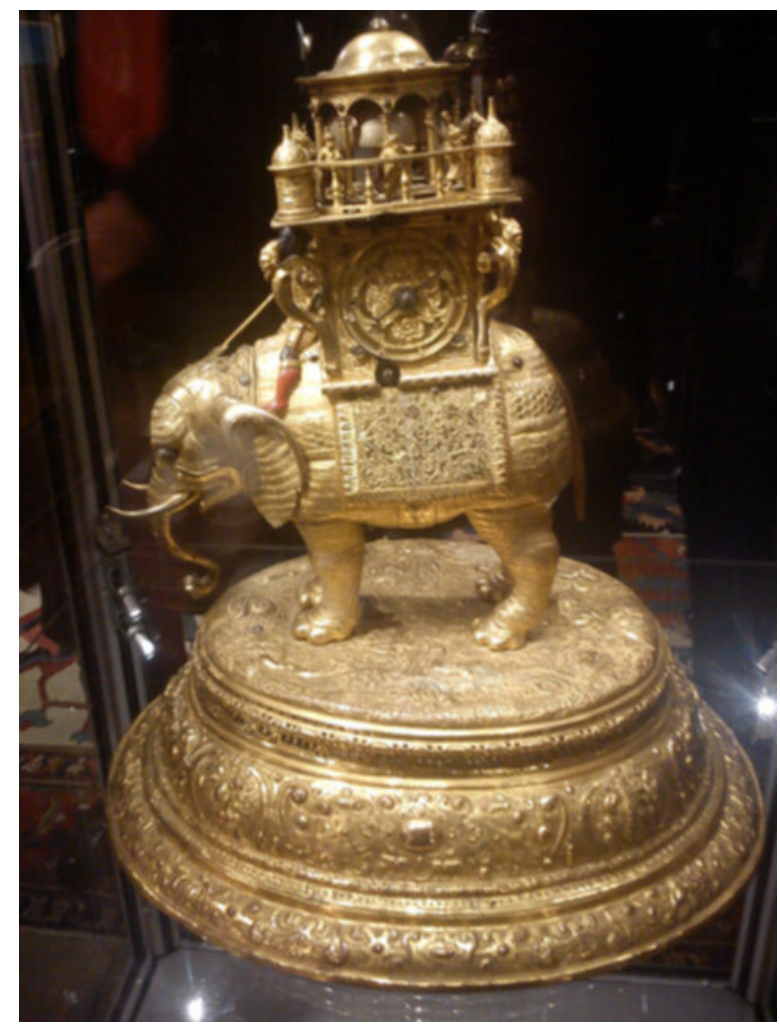

Question:are there any people in the image?

MRR candidate set

1. no

2. no people

3. nope

4. no i do not see people

5. not that i can see

\section{Top 10 from the remaining NDCG candidates}

1. noo

2. 0 are visible
3. 0 i can see

4. 0

5. nobody around

6. 0 sen

7. do n't really see any

8. $\mathrm{mp}$

9. i do n't think so

10. not really

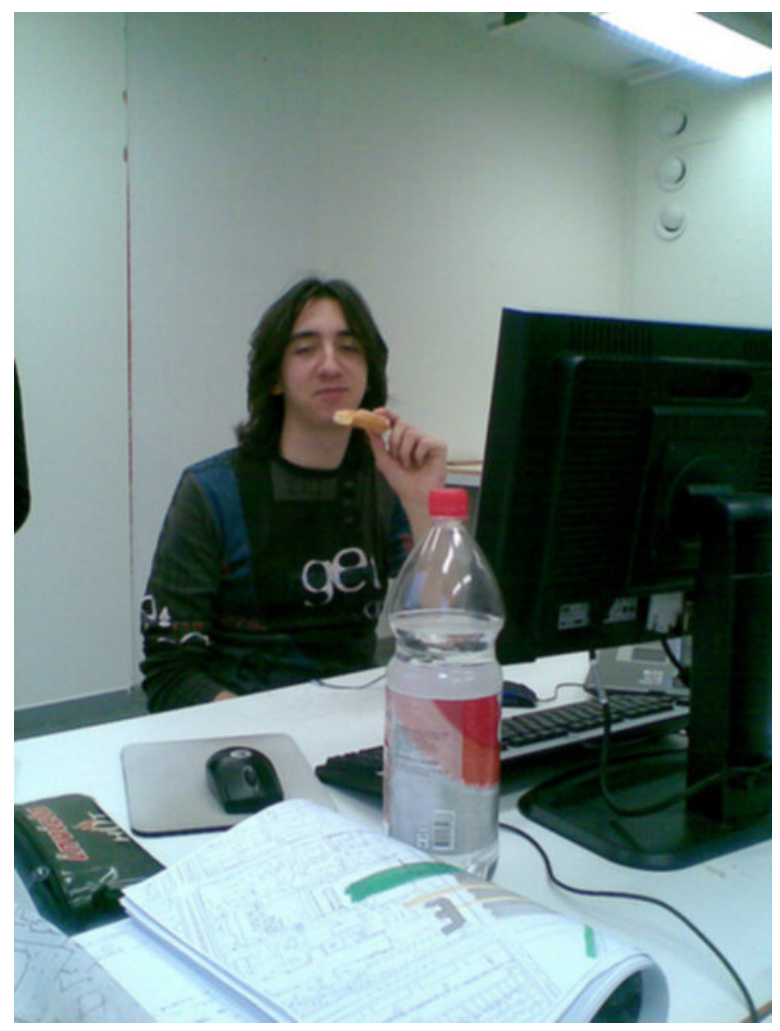

Question:is this a living room?

\section{MRR candidate set}

1. no

2. yes

3. ca n't tell

4. i do n't think so

5. i ca n't tell

6. nope

\section{Top 10 from the remaining NDCG candidates}

1. i do not think so

2. ca n't really tell 
3. can not tell

4. i can not tell

5. i do n't know

6. i ca n't tell for sure

7. not sure

8. i don' 't know

9. hard to tell

10. not that i can see

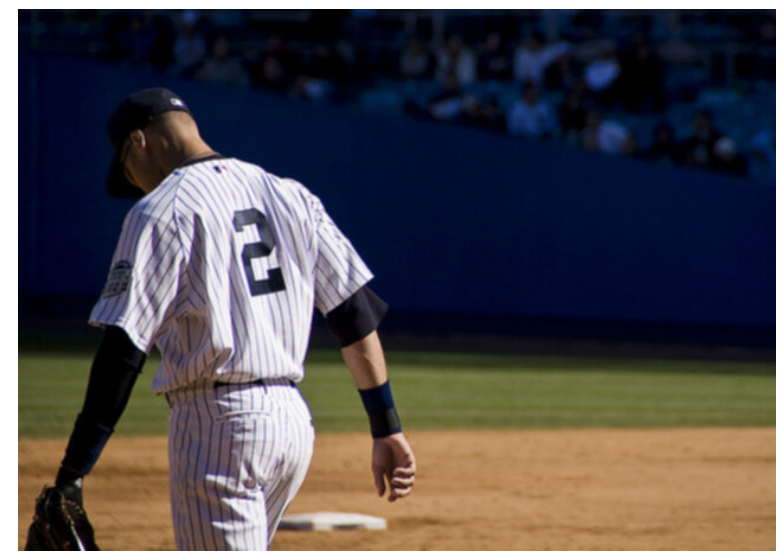

Question:what color is the jersey?

MRR candidate set

1. pinstripe white

2. white

3. it is white

4. blue and white

5. it's white

\section{Top 10 from the remaining NDCG candidates}

1. grey

2. photo is in black and white

3. the picture is in black and white

4. the pants are gray and his jersey is navy blue

5. its a black and white picture so i' $\mathrm{m}$ not sure

6. we can say that

7. it's blue

8. grey and UNK cloudy

9. beige and blue
10. the die is a grey and white $i$ believe

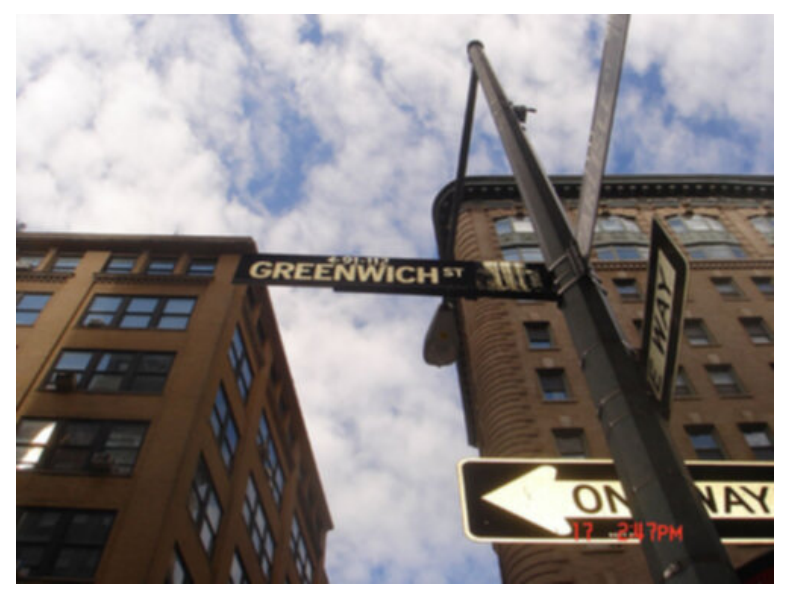

Question:are they tall ?

\section{MRR candidate set}

1. yes, they are tall

2. yes

3. yes they are

4. yes they definitely are

\section{Top 10 from the remaining NDCG candidates}

1. yes a few stories at least

2. looks like

3. yes, some are

4. i think so

5. not really

6. yes, it is

7. no

8. yes it is

9. nope

10. possibly

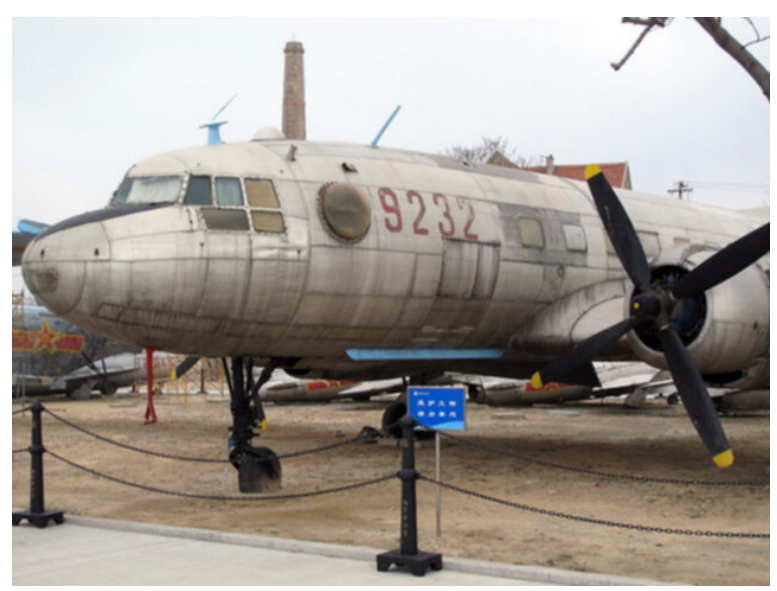


Question:does it look rusty?

MRR candidate set

1. no

2. yes

3. not at all

4. nope

Top 10 from the remaining NDCG candidates

1. not really

2. no it is not

3. kind of

4. no but looks like it did previously

5. yes, it looks UNK

6. not that i can see

7. yes, it does

8. i do n't think so

9. yeah

10. sort of

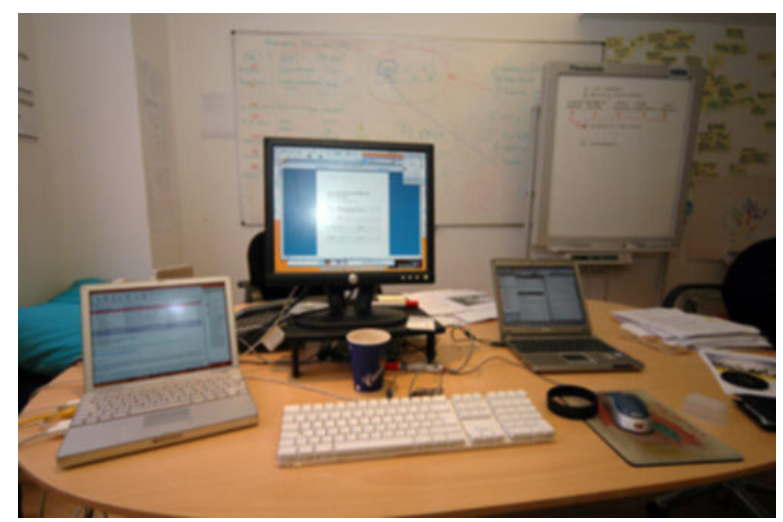

Question:is this inside?

\section{MRR candidate set}

1. yes

2. yes it is

3. yes, it is

4. yes it appears to be

5. i think so

Top 10 from the remaining NDCG candidates

1. yes it 's indoors
2. i believe so

3. yes* and yes

4. appear to be

5. most likely indoors

6. it might be

7. $\mathrm{i}$ believe so but it is hard to tell for sure

8. most

9. yes it 's clean

10. maybe

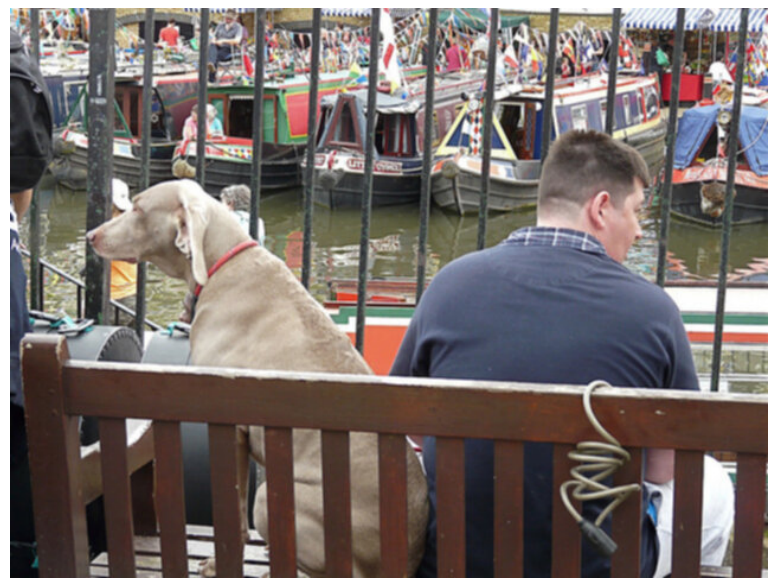

Question:how many other people are there ? MRR candidate set

1. 2 people

2. a crowd

3. lots and lots

4. very many

5. approximately 20

Top 10 from the remaining NDCG candidates

1. it looks like over 20

2. 15 maybe

3. 10

4. about a 100

5. about 10

6. ca n't tell

7. 8 or so

8. 12 or so 
9. around 10

10. i can not tell

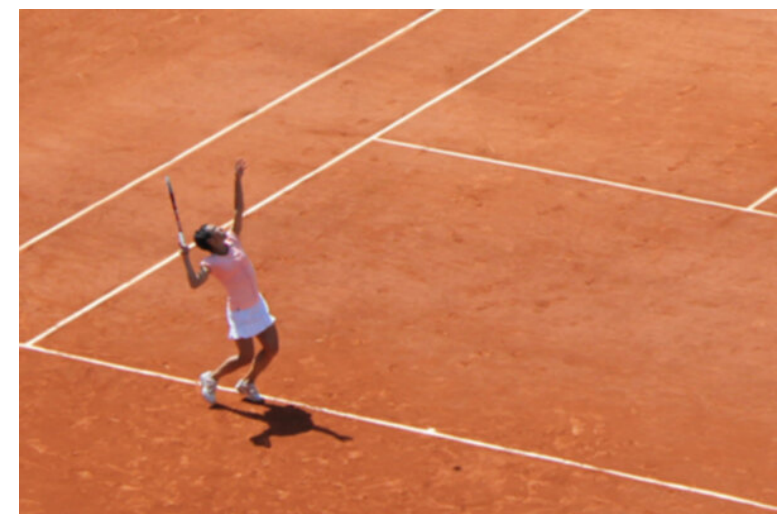

Question:is she wearing shorts?

MRR candidate set

1. no a white skirt

2. yes

3. no, she is wearing a skirt

4. no

5. yes she is

6. i think so

\section{Top 10 from the remaining NDCG candidates}

1. white

2. hard to tell, but i think so

3. nope

4. yes, it is

5. can

6. yes, they are

7. yes he is

8. yes it is

9. he

10. maybe

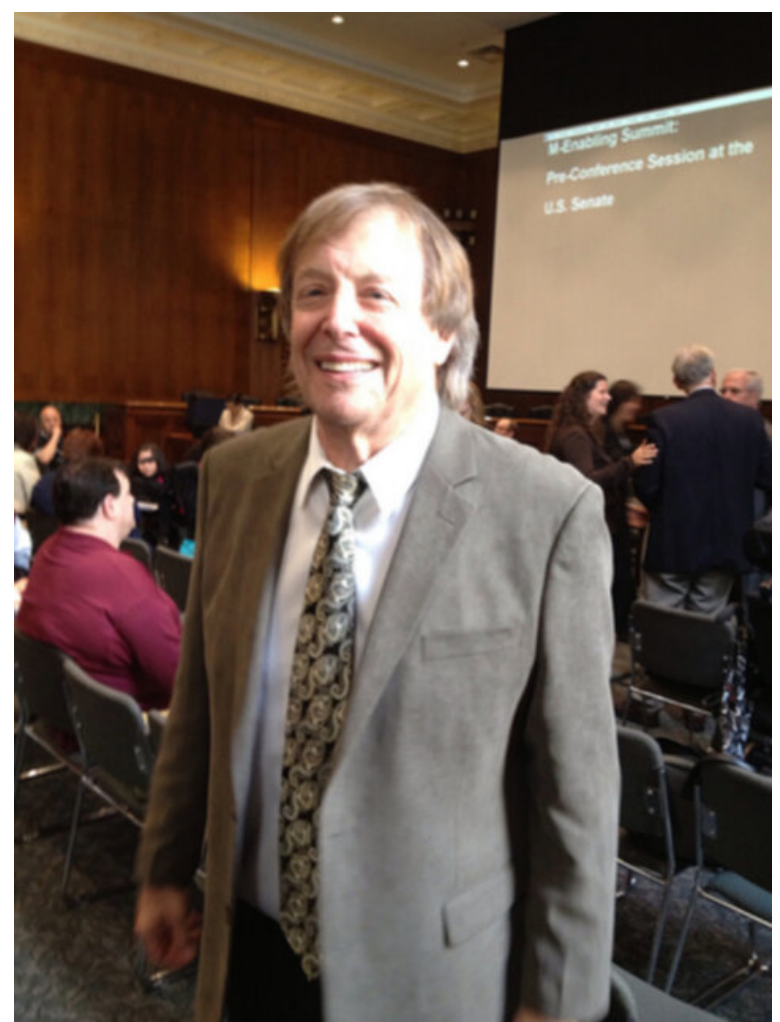

Question:what type of flooring? MRR candidate set

1. carpet

2. tile

3. i can not tell

4. i ca n't tell

5. ca n't tell

\section{Top 10 from the remaining NDCG candidates}

1. not sure

2. i don' t know for sure

3. maybe tan

4. ca n't tell from the picture

5. no idea

6. brown

7. grey

8. seem grayish from this angle

9. green

10. color of what 


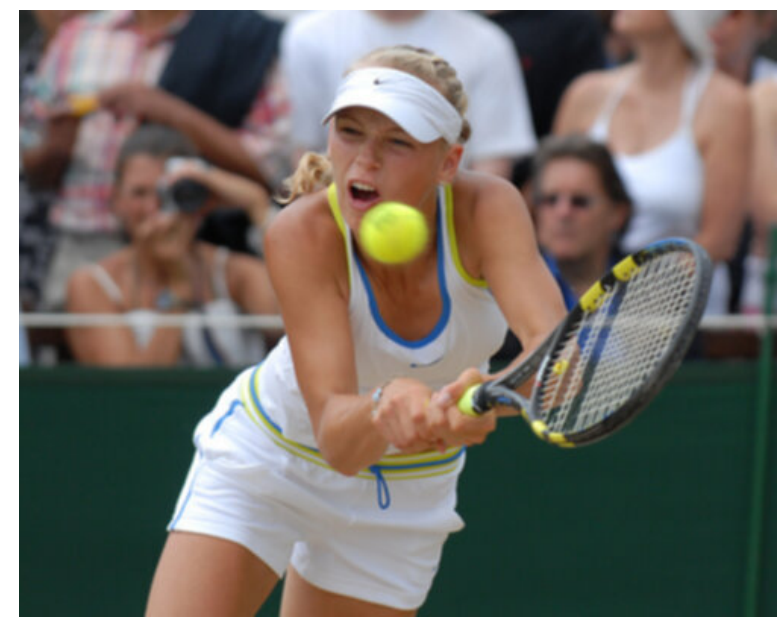

Question:cab you tell the women 's race? MRR candidate set

1. caucasian

2. white

3. no i can not

4. no i ca n't

Top 10 from the remaining NDCG candidates

1. no

2. no i do not

3. nope

4. yes

5. i can not tell

6. not sure

7. not really

8. yes, i can

9. yes i can

10. i ca n't tell

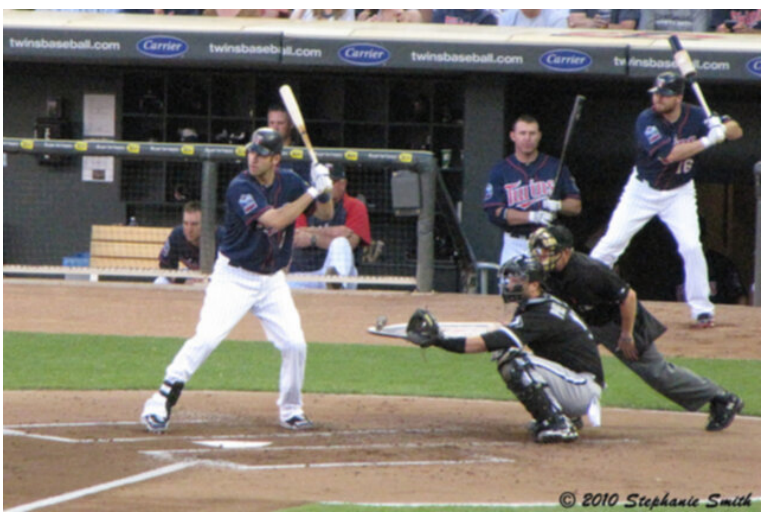

Question:can you see the scoreboard?

MRR candidate set
1. no

2. yes

3. i can not

4. nope

5. no i can not

Top 10 from the remaining NDCG candidates

1. no it is cut off

2. not really

3. $\mathrm{n}$

4. i do n't think so

5. not that $\mathrm{i}$ can see

6. i can not tell

7. not sure

8. barely

9. i ca n't tell

10. ca n't tell

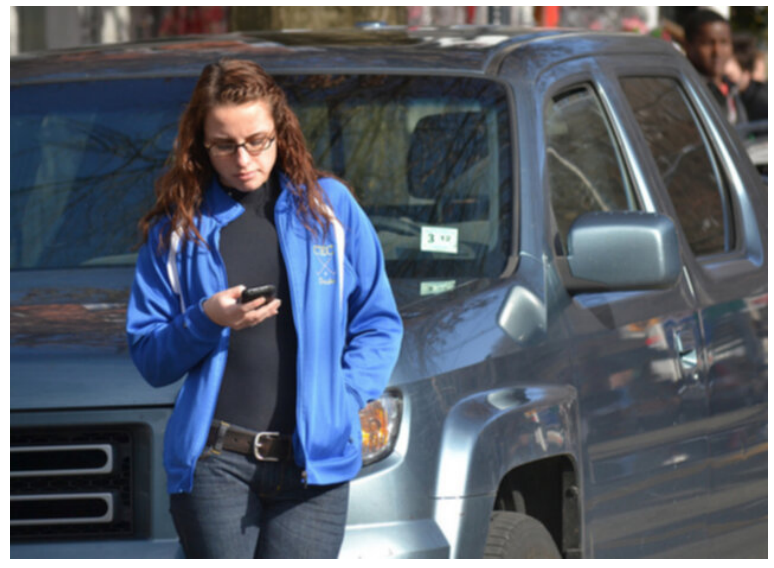

Question:is she wearing sneakers ?

MRR candidate set

1. no

2. not visible

3. i ca n't see her feet

4. ca n't tell

5. i can not tell

6. i ca n't tell

Top 10 from the remaining NDCG candidates 
1. ca n't know

2. nope

3. i ca n't tell from the picture

4. can

5. not sure

6. i do n't think so

7. not that $\mathrm{i}$ can see

8. ca n't see the bottom part

9. maybe $\mathrm{i}$ am not sure

10. yes

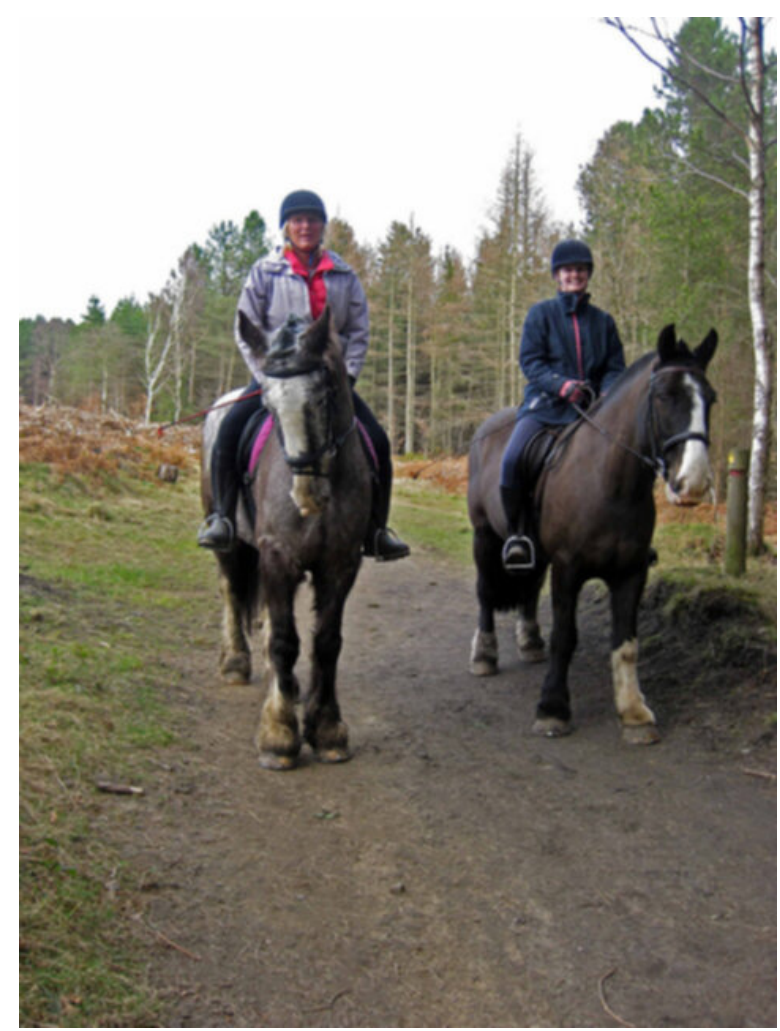

Question:is it sunny outside ?

\section{MRR candidate set}
1. yes
2. no
3. not really
4. no it is not

\section{Top 10 from the remaining NDCG candidates}

1. no, it is cloudy

2. it appears sunny
3. no, it is not sunny outside

4. overcast

5. nope

6. it looks cloudy

7. no cloudy

8. it 's somewhat overcast

9. yes it is sunny

10. yes, looks sunny

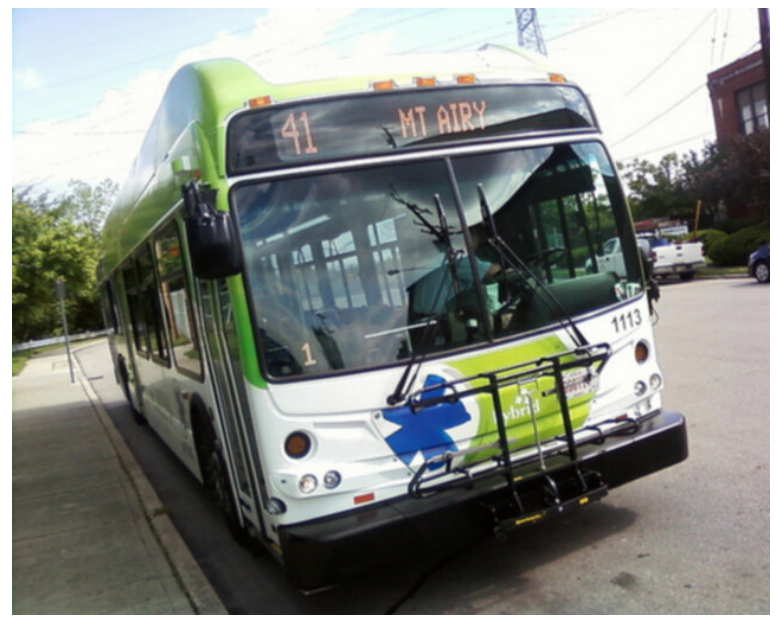

Question:are they in a parking lot or on the side of the road?

\section{MRR candidate set}

1. on the side of the road

2. yes

3. ca n't tell

4. i ca n't tell

5. i can not tell

6. not sure

Top 10 from the remaining NDCG candidates

1. it 's hard to tell, but i can not see any

2. yes , many of them are lined up on the sidewalk

3. looks like it, ca n't tell

4. 1 is, yes

5. no, just 1

6. yes, it is 
7. i think so

8. no, they are not

9. on the ground

10. yes it is a side view only

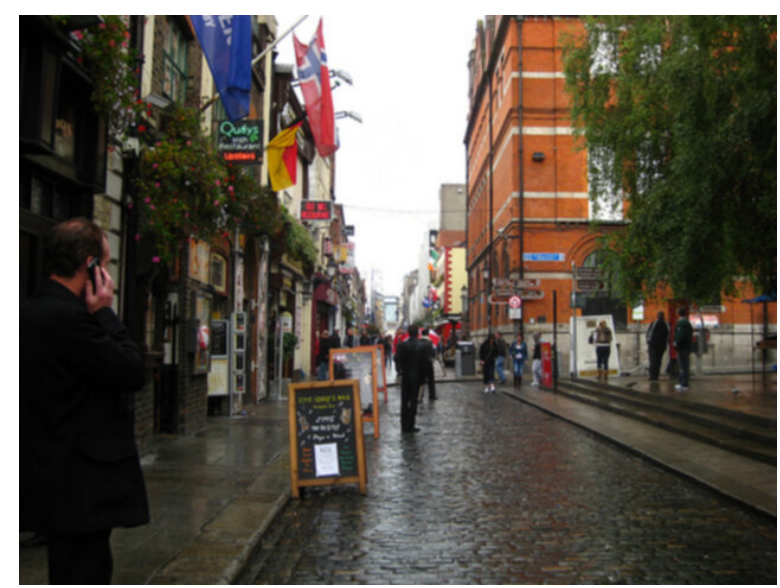

Question:is he holding it to his ear or using UNK ?

\section{MRR candidate set}

1. it 's up to his ear

2. ca n't tell

3. i ca n't tell

4. i can not tell

Top 10 from the remaining NDCG candidates

1. ca n't see

2. not sure

3. not that $i$ can see

4. can you see

5. no

6. you ca n't tell

7. nope

8. not really it 's kind of far away

9. both

10. yes

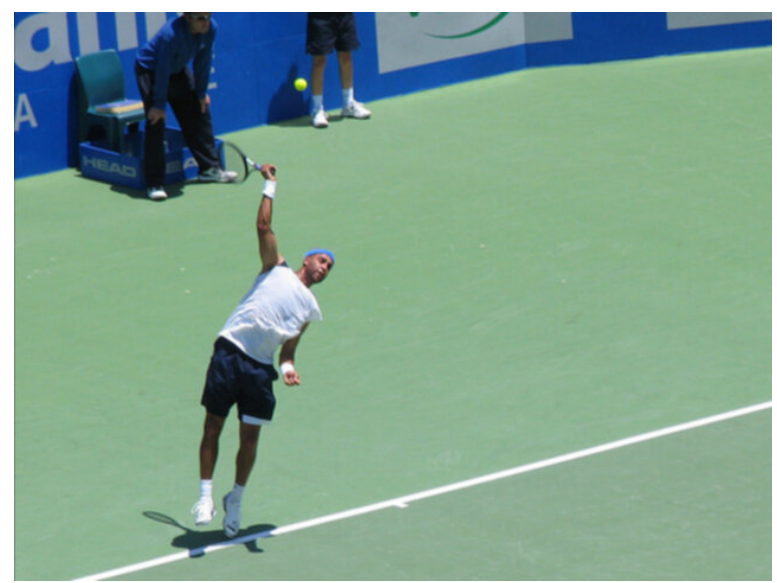

Question:is the man wearing a visor ?

MRR candidate set

1. no

2. yes

3. no heat, but maybe more of a sweatband

4. he is not

5. nope

6. no he is not

\section{Top 10 from the remaining NDCG candidates}

1. i do n't think so

2. yes, he does

3. yes he is

4. not that $\mathrm{i}$ can see

5. yes!

6. $\mathrm{i}$ believe so

7. yep

8. ca n't see that

9. can

10. i think so

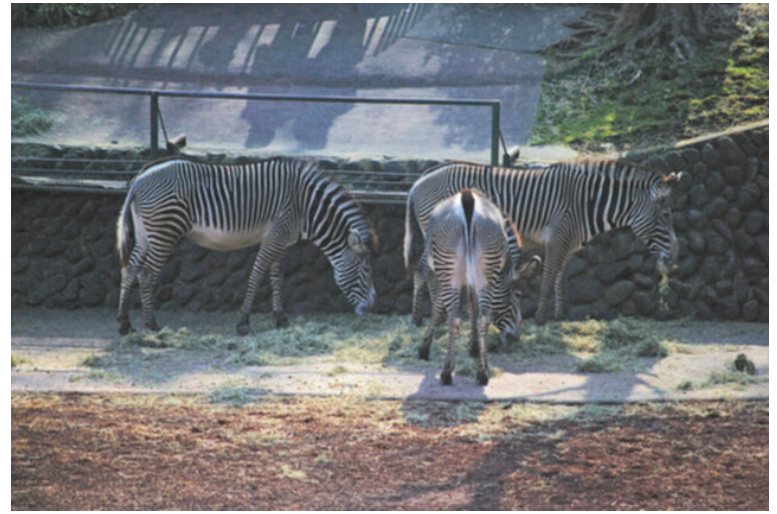


Question:could this be a zoo ?

MRR candidate set

1. yes

2. maybe

3. i think so

4. i would think so

5. ca n't tell if it is

\section{Top 10 from the remaining NDCG candidates}

1. looks like it

2. it looks like it

3. ca n't tell

4. possibly, not sure

5. i ca n't tell

6. possibly, ca n't really tell

7. it looks like it is

8. not sure

9. they appear to be

10. it 's hard to tell

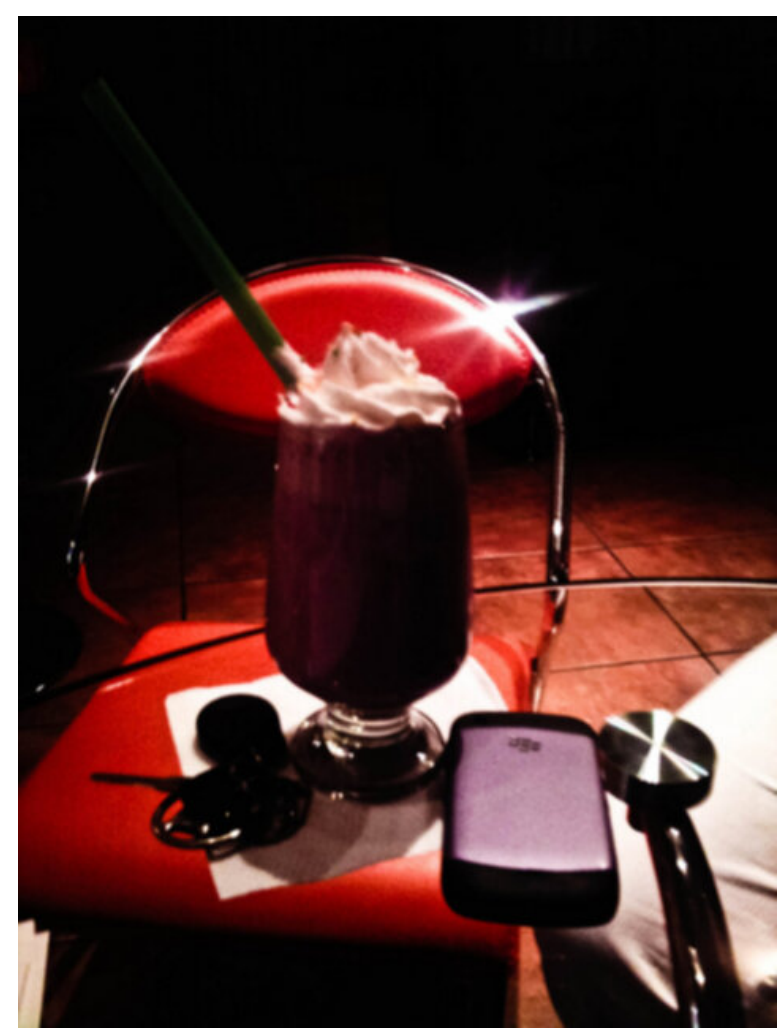

Question:is this a smartphone?

MRR candidate set
1. yes

2. no

3. ca n't tell

4. i ca n't tell

5. i do n't know

\section{Top 10 from the remaining NDCG candidates}

1. i can not tell

2. not sure

3. hard to tell

4. nope

5. it does n't appear to be

6. i do not think so

7. no it is n't

8. i do n't think so

9. ca n't tell , maybe

10. no is not

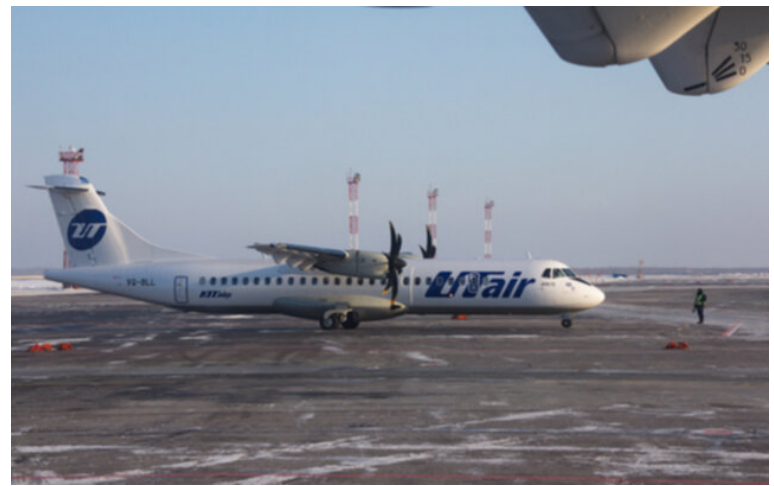

Question:are there words on the plane?

MRR candidate set

1. yes, the plane says UNK

2. yes

3. yes, there are words

4. yes, there are

5. yes there are

6. yes there is

Top 10 from the remaining NDCG candidates

1. yes, it says bp 
2. yes, quite a few

3. some yes

4. yes, it is

5. i think so

6. it appears to be a website i see words and an image but ca n't make them out

7. yes, i see some from the window

8. yes , many windows

9. yes, water

10. yes it is

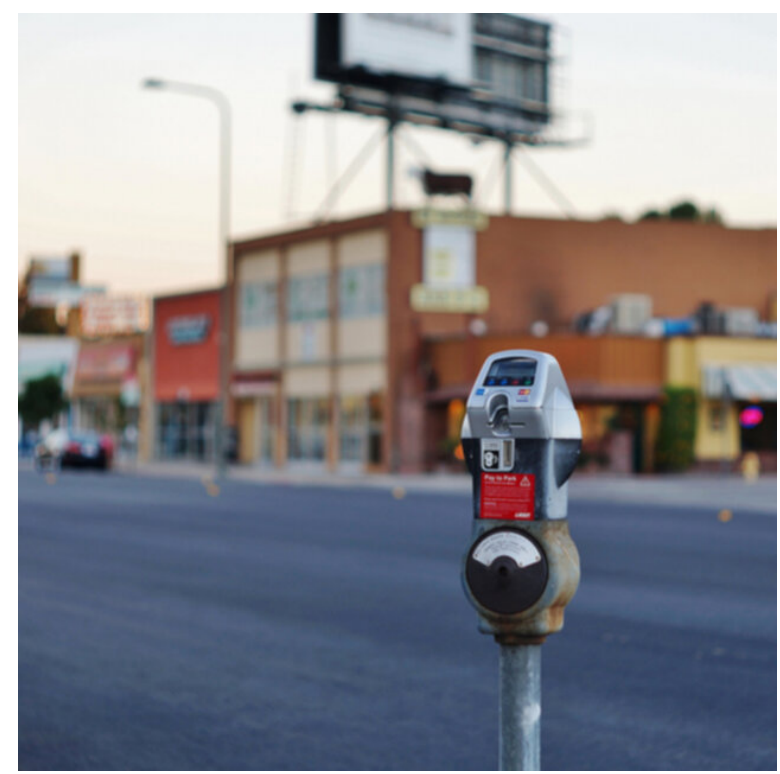

Question:can you see any store signs?

\section{MRR candidate set}

1. no, it is blurry

2. no

3. yes

4. no i do n't think so

5. nope

6. not that $i$ can see

\section{Top 10 from the remaining NDCG candidates}

1. there are 0 of those

2. in the distance

3. yes in the background

4. not really
5. yes, several in the background

6. i do n't think so

7. yes some

8. barely

9. yes quite a few in the background

10. 1 partially off in the distance

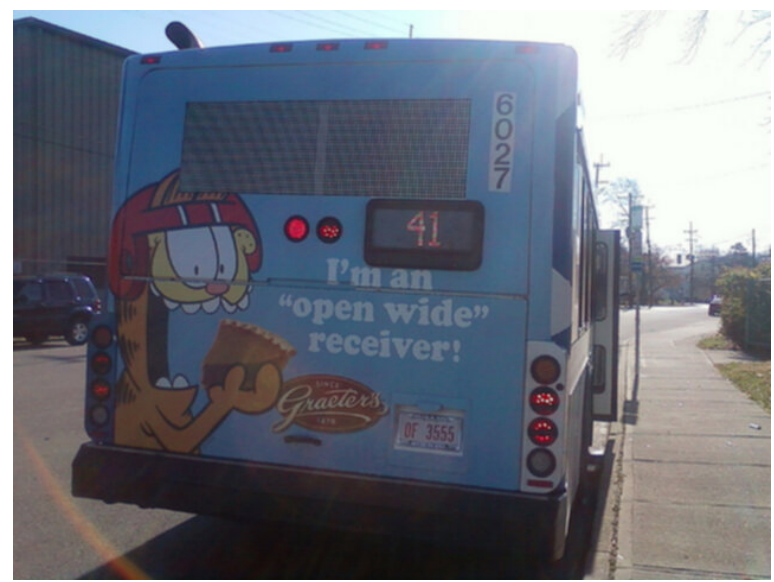

Question:can you see inside the bus? MRR candidate set

1. no, just the back of the bus

2. no

3. i can not

4. i can not tell

5. no i ca n't

6. nope

Top 10 from the remaining NDCG candidates

1. no'

2. no, i do n't

3. not really

4. yes

5. barely

6. i do n't think so

7. yes, i can

8. not it 's not visible

9. not that i can see

10. i ca n't tell 


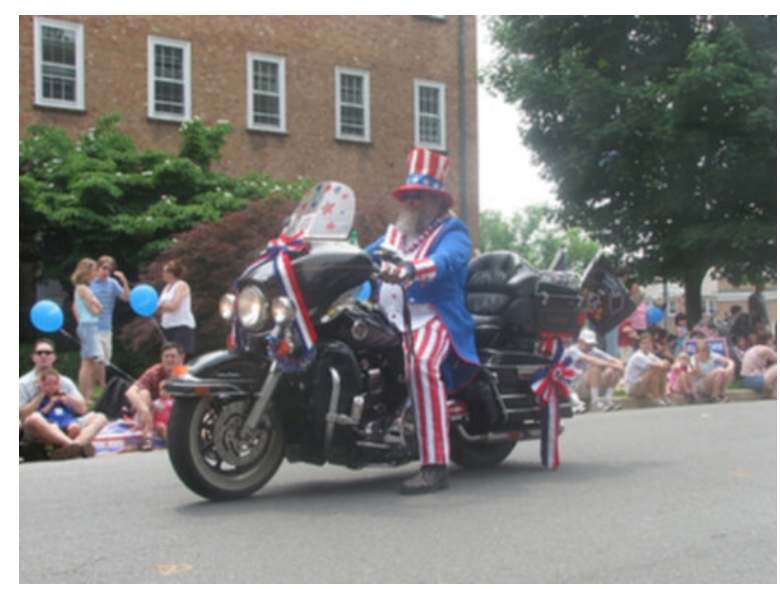

Question:is the man wearing a mask?

MRR candidate set

1. no

2. yes

3. no he does not

4. no, he is not

5. nope

Top 10 from the remaining NDCG candidates

1. i do n't think so

2. does n't look like it

3. not that i can see

4. not really

5. no, he 's wearing a helmet

6. yes indeed

7. he is

8. yes he is

9. i do n't think he is, hard to tell with most of his head down

10. it looks like a helmet

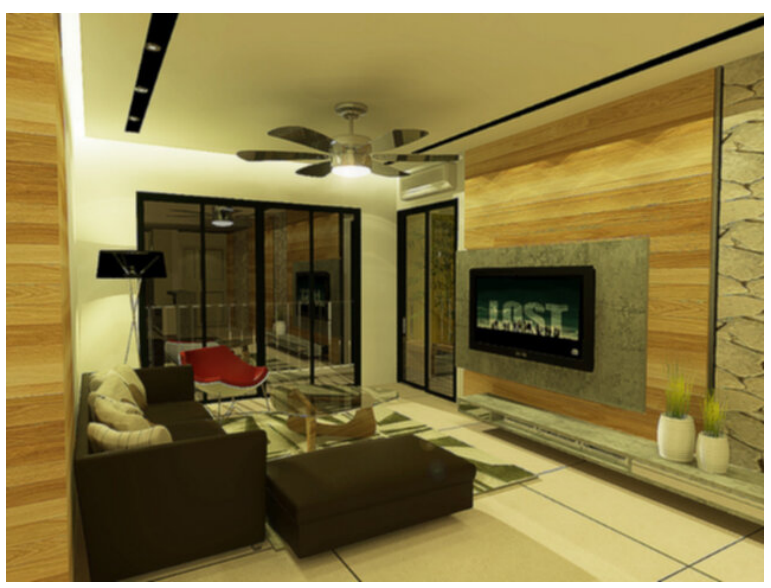

Question:does the room look big?

MRR candidate set

1. it is decent size

2. yes, it does

3. yes

4. i 'd say so

5. yes, very

\section{Top 10 from the remaining NDCG candidates}

1. looks like it

2. it looks like

3. not too big

4. no, it is pretty small

5. yes, it is

6. average

7. it could be

8. i think it

9. not really

10. very

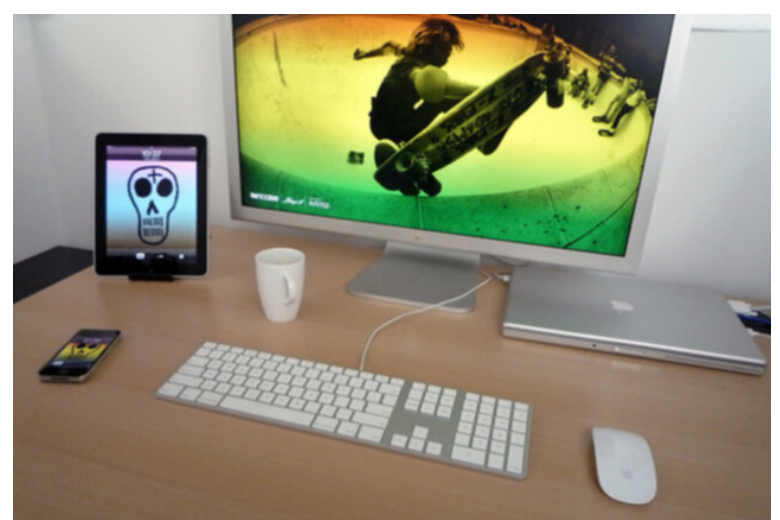

Question:is the laptop on a desk ?

MRR candidate set

1. yes

2. a table

3. yes it is

4. yes, it is

5. it appears to be

6. yes it looks like it is 


\section{Top 10 from the remaining NDCG candidates}

1. looks like it

2. i think so

3. on a portable desk thing, like a lap desk

4. yes it is on

5. yes, just 1

6. no, it looks like it is on a table

7. no on a stand

8. no a stand

9. yes, he she is

10. yes, there is something below the tv

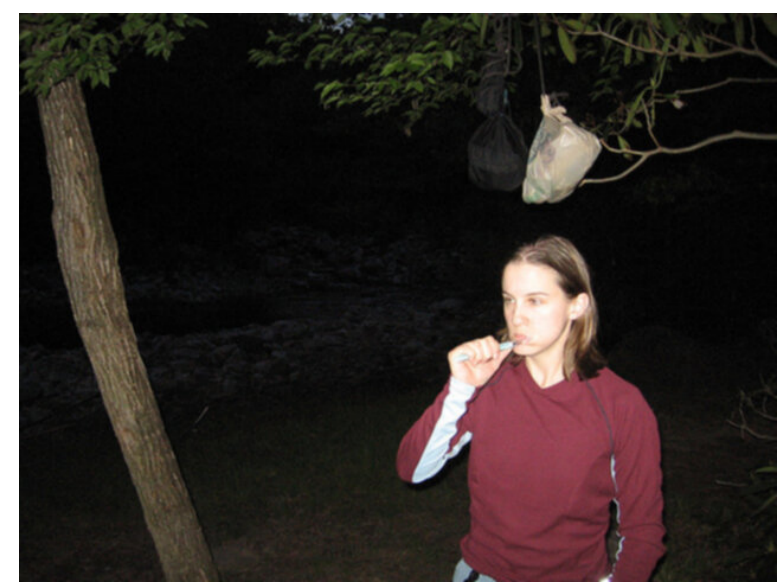

Question:what color is her hair?

\section{MRR candidate set}

1. brown

2. blonde

3. dark blonde

4. it's brown

5. her hair is brown

6. she has brown hair

\section{Top 10 from the remaining NDCG candidates}

1. dark brown

2. dirty blonde

3. dark, brown

4. i think it brown

5. brown, i think
6. it 's dirty blonde

7. black

8. red

9. UNK rose UNK

10. looks dirty

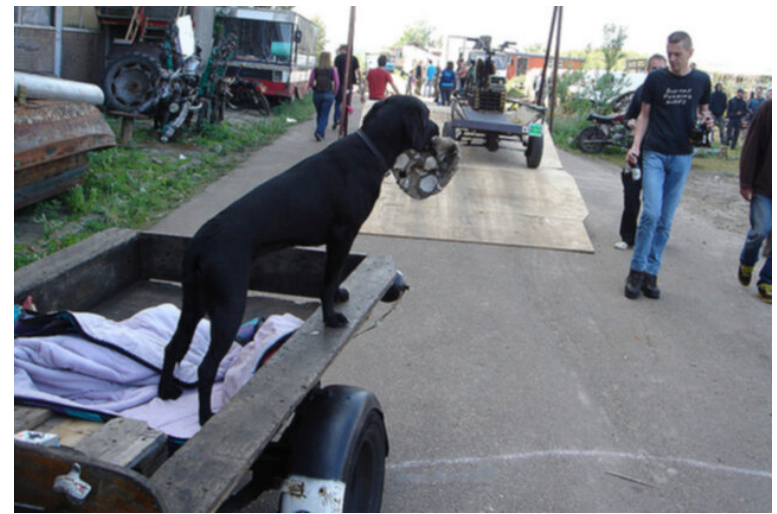

Question:what color is the dog ? MRR candidate set

1. black

2. black lab

3. the dog is black

4. it is black

5. dark

Top 10 from the remaining NDCG candidates

1. black and white

2. dark brown, beautiful

3. it 's dark

4. not sure

5. black and brown

6. i can not tell

7. 1 is black, and the other is brown

8. it 's in a shadow but maybe black

9. black and white feet

10. i ca n't tell 


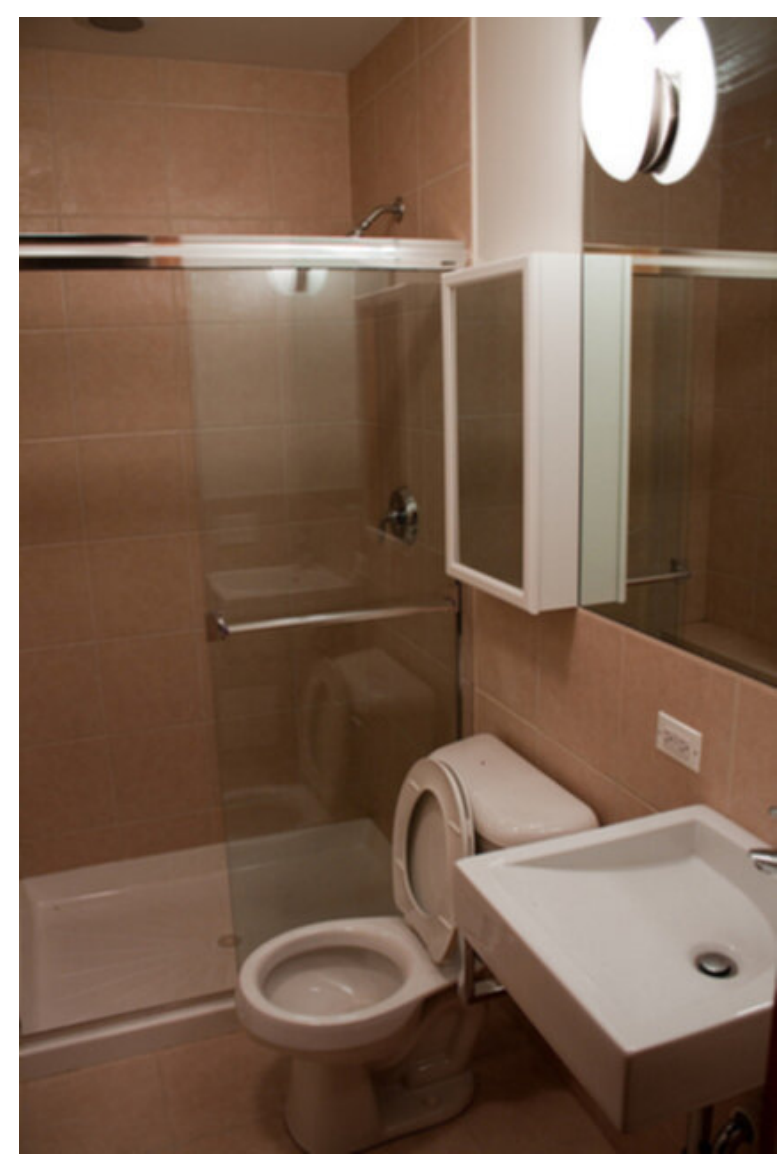

Question:any walls?

MRR candidate set

1. yes

2. yes, it is

3. yes a large 1

4. yess

\section{Top 10 from the remaining NDCG candidates}

1. yes 1

2. yes it is

3. hello it 's mostly brown

4. UNK

5. yes, to the left

6. i think so

7. no

8. barely part of 1

9. is pink

10. not that i can see

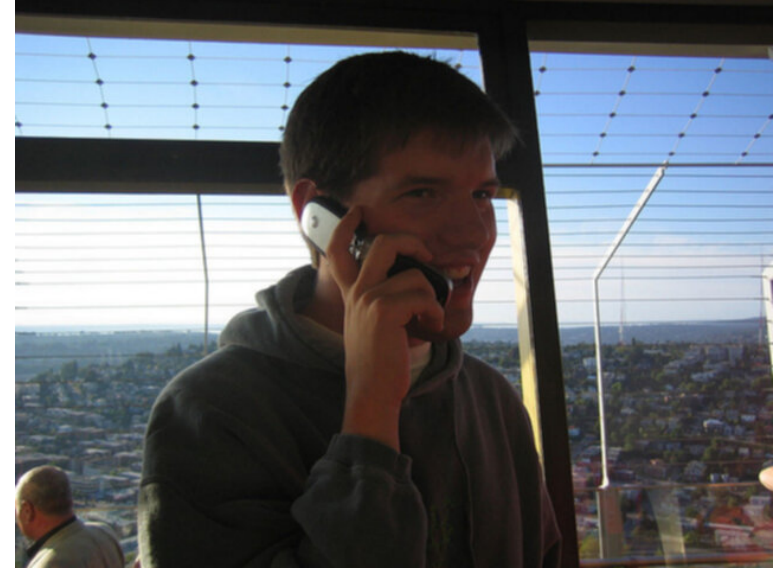

Question:are the buildings tall ?

MRR candidate set

1. it is hard to tell from the picture

2. i can not tell

3. in the background barely visible

4. i ca n't tell

5. hard to tell

6. ca n't tell

\section{Top 10 from the remaining NDCG candidates}

1. they do not appear to be

2. possible hard to tell

3. yes, they appear to be

4. not sure

5. yes

6. possibly

7. no

8. i do n't think so

9. not really

10. i think so

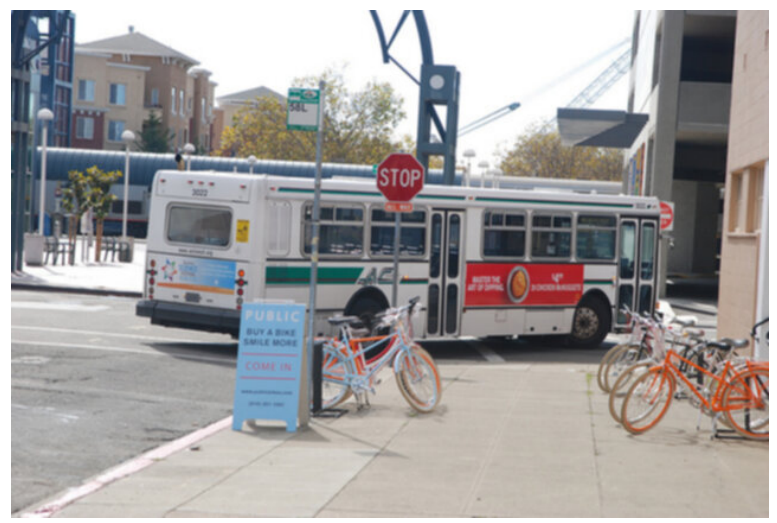


Question:can you see any trees ?

\section{MRR candidate set}

1. yes

2. yes in the background

3. yes, a few in the background

4. yes, in the background

5. yep

\section{Top 10 from the remaining NDCG candidates}

1. yes some

2. in the distance, a few

3. in the background

4. there are several in the background

5. there are a few large trees near by and several in background

6. yes, there are a lot of trees

7. ues

8. some

9. yes, far in the background

10. yes 1

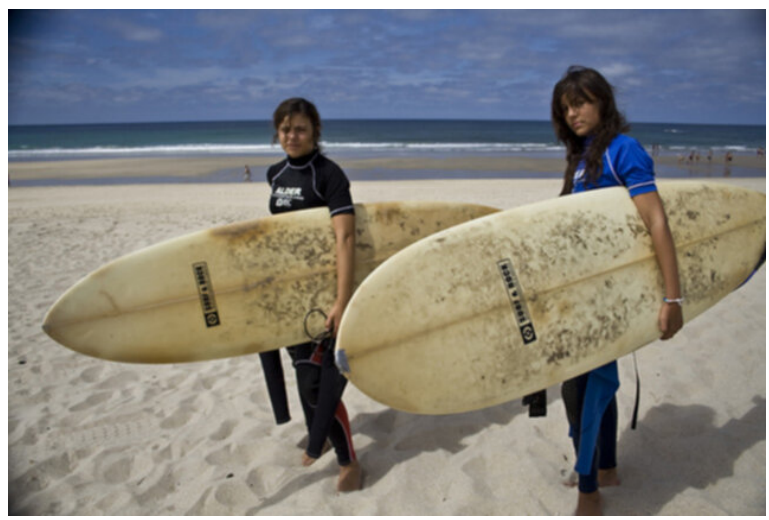

Question:what is the gender of the people?

\section{MRR candidate set}

1. both boys

2. female

3. hard to say maybe male and female

4. not sure

Top 10 from the remaining NDCG candidates

1. they look caucasian
2. looks male

3. yes

4. it appears so

5. ca n't tell

6. they are both posing for the picture 1 posing as if he 's going to hit the tennis ball the

7. i can not tell

8. no 1 is on 1 st, 2 nd and 3 rd are not visible

9. i ca n't tell

10. yes, it is

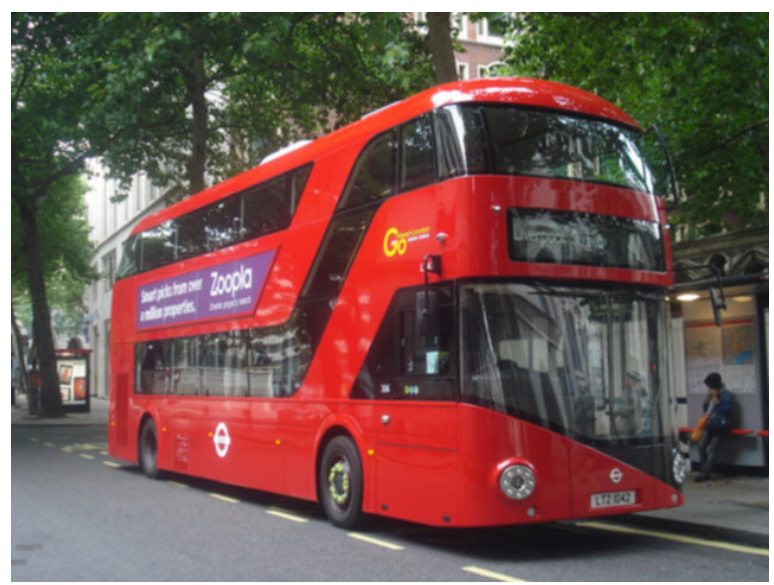

Question:does the bus have a number?

MRR candidate set

1. no

2. yes

3. i ca n't see 1

4. no that $i$ can see

5. yes it does

Top 10 from the remaining NDCG candidates

1. yes,

2. a number pad yes

3. not that i can see

4. nope

5. do n't see 1

6. i think so

7. does n't look like it 
8. can not tell not visible

9. no it does n't

10. 2

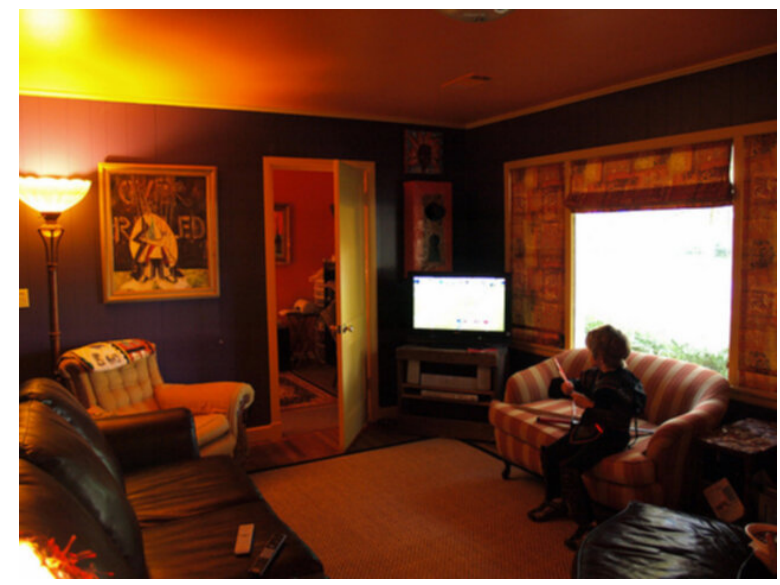

Question:what kind of toy is the boy playing with $?$

\section{MRR candidate set}

1. can not tell which toy it is

2. look to be a wii video game

3. i can not tell

4. i ca n't tell

5. ca n't tell

6. i m not sure

\section{Top 10 from the remaining NDCG candidates}

1. you can not see who he is playing with

2. i do n't know

3. a small remote controlled 1

4. not sure

5. i see 1 , but its far

6. a tiny bird

7. kind of a derby i think it 's called

8. i ca n't see the ball

9. a bowl

10. maybe a UNK

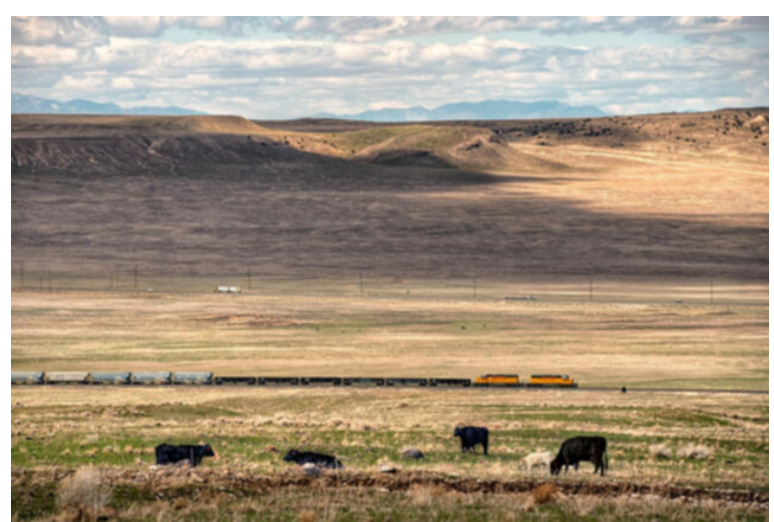

Question:is the sky cloudy? MRR candidate set

1. yes

2. yes, blue skies some clouds

3. yes it is

4. yes, it is

\section{Top 10 from the remaining NDCG candidates}

1. yes'

2. yes but it is blue sky

3. yeah pretty much

4. yes, it is very clear

5. yes very clear

6. yes partially

7. i think so

8. it appears overcast

9. a little bit

10. relatively

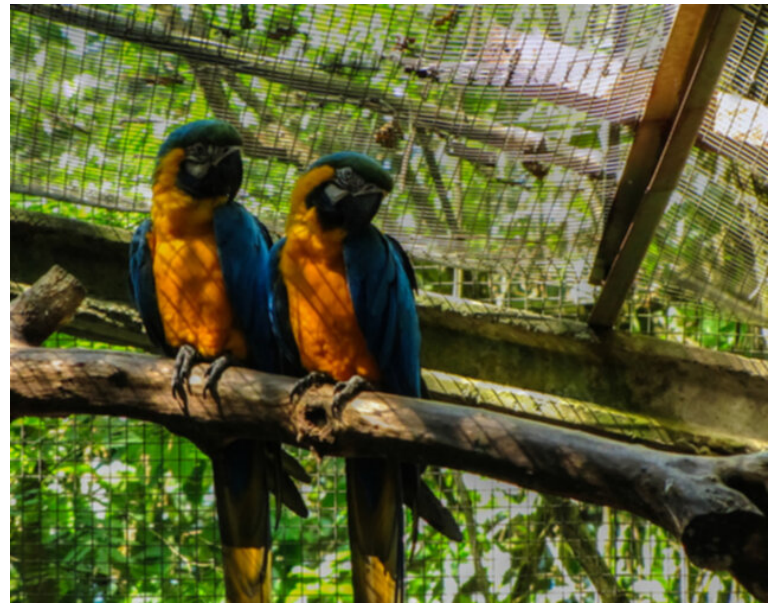

Question:is the cage made of metal ? MRR candidate set 
1. yes

2. metal and wood - looks like a zoo enclosure

3. yes it is

4. it appears to be

5. yes, it is

\section{Top 10 from the remaining NDCG candidates}

1. yes it is,

2. i think so

3. уep

4. wood

5. hmm i think so

6. no

7. i ca n't really tell

8. they are and have metal parts too

9. i ca n't tell

10. i can not tell

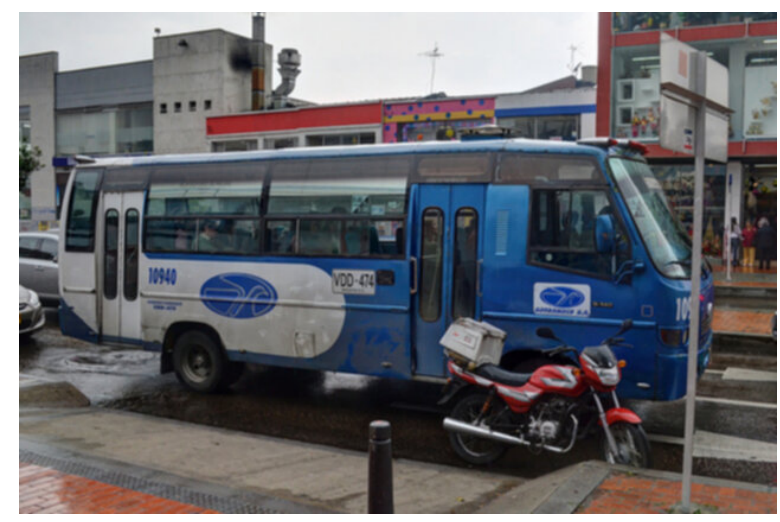

Question:do any of them have their hair up ? MRR candidate set

1. no

2. not that i can see

3. no they do n't

4. i ca n't tell

5. nope

\section{Top 10 from the remaining NDCG candidates}

1. ca n't tell

2. i do n't think so
3. i can not tell

4. i do n't know

5. not sure

6. too far away in photo to tell

7. hard to tell since they 're far away

8. the picture does not show 1

9. 0

10. maybe, not sure

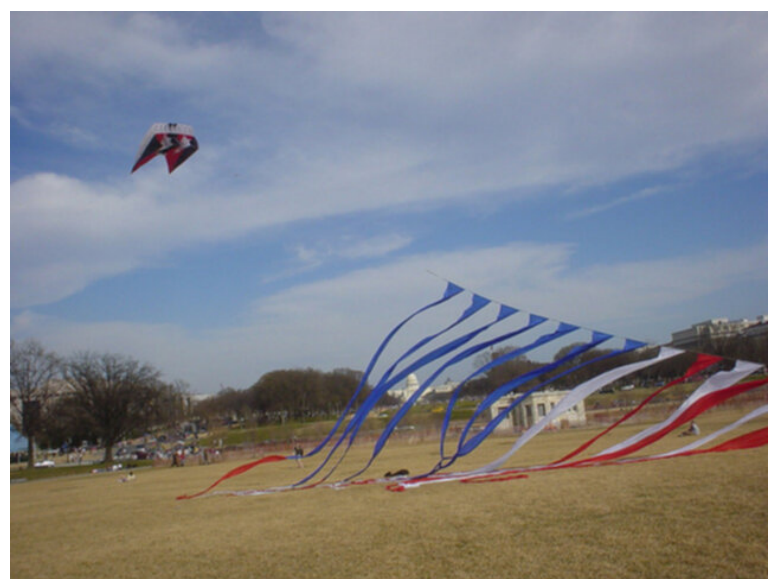

Question:is there other kites in the sky or just the 1 ?

MRR candidate set

1. there is 1 other kite

2. 1

3. i can only see 1 in this picture

4. not that $\mathrm{i}$ can see

\section{Top 10 from the remaining NDCG candidates}

1. no there are n't

2. no

3. nope

4. yes a few

5. yes

6. just beside it

7. yes, both

8. no , no 1 else in the photo

9. in the air 
10. no just her

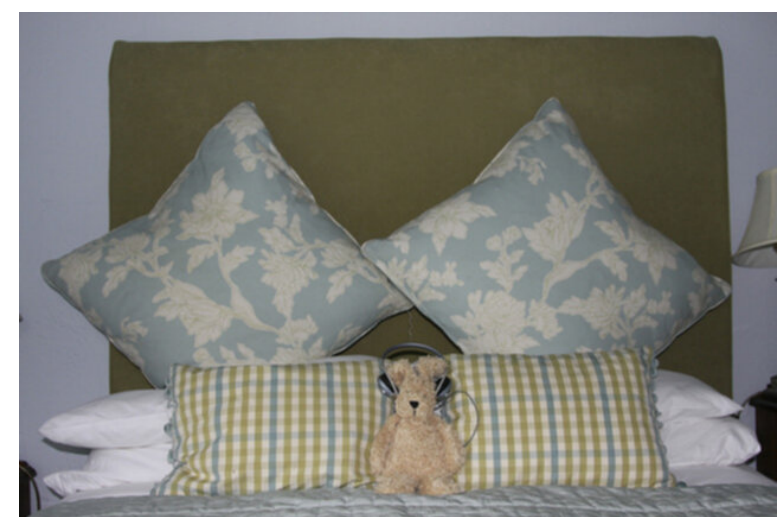

Question:is it a close up of the bear? MRR candidate set

1. yes

2. yes, it is

3. yes, pretty close

4. yes it is

5. it is, yes

\section{Top 10 from the remaining NDCG candidates}

1. sort of

2. i think so

3. it look like it

4. a little bit, yes

5. no

6. not really

7. yes there is

8. nope

9. yes, it is very clear

10. a few inches or so

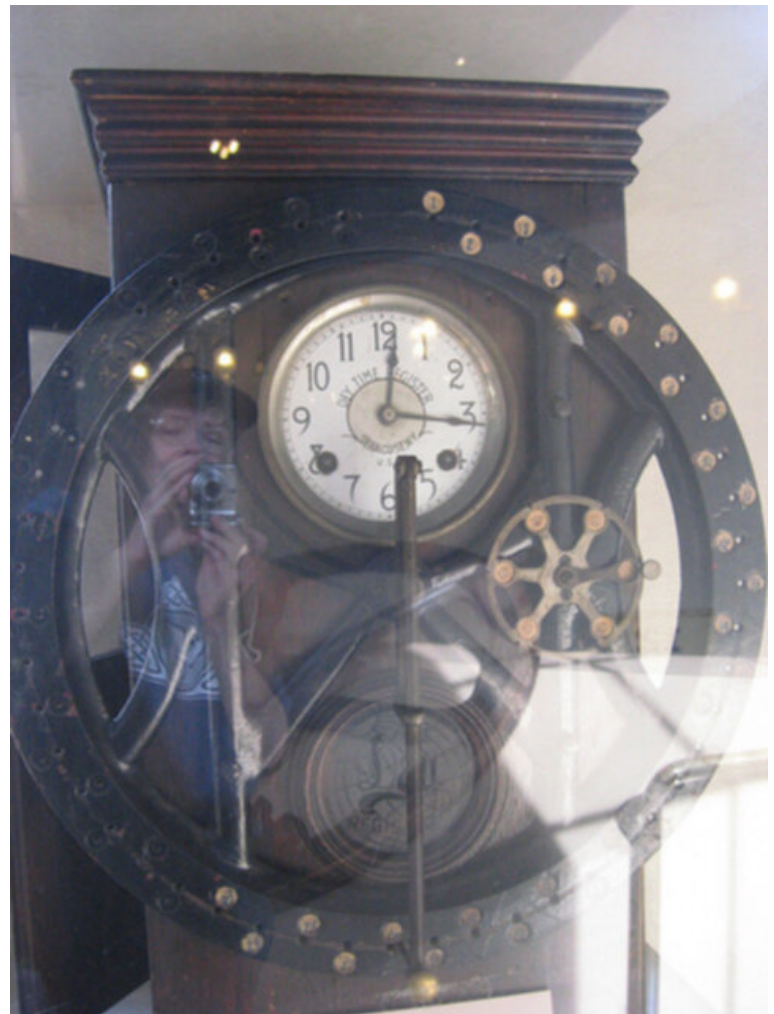

Question:is the shelf wood? MRR candidate set

1. yes

2. i can not tell

3. yes, it is

4. i think so

5. yes, i think so

6. yes it is

\section{Top 10 from the remaining NDCG candidates}

1. no

2. looks like it

3. no it's not

4. ca n't tell

5. i ca n't tell

6. $\mathrm{i}$ think it is, but hard to say for sure

7. not sure

8. it is hard to tell

9. yes it is brown

10. i do n't think so 


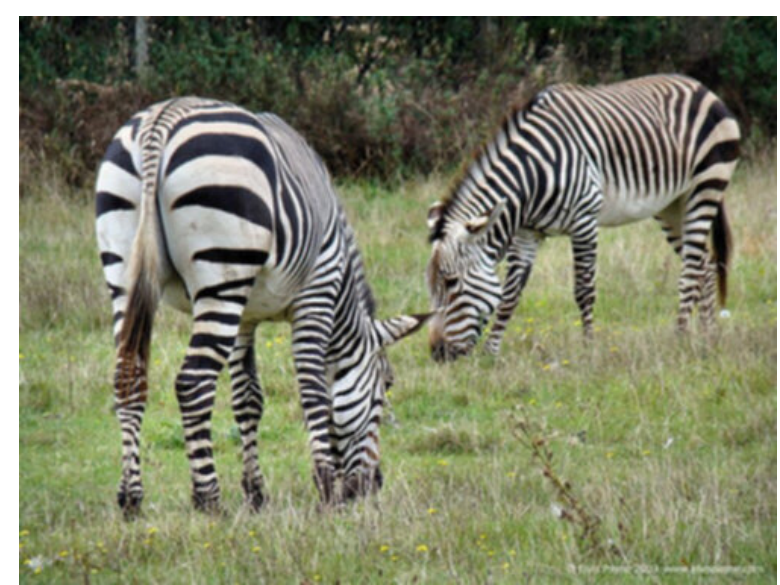

Question:can you see any roads?

\section{MRR candidate set}

1. no

2. nope

3. no i ca n't

4. i do n't

5. not that $i$ can see

Top 10 from the remaining NDCG candidates

1. no you can not

2. 0

3. $\mathrm{np}$

4. i do n't think so

5. not really

6. nobody around

7. no, there are no signs

8. no buildings

9. no, it 's sunny

10. no it 's too far away

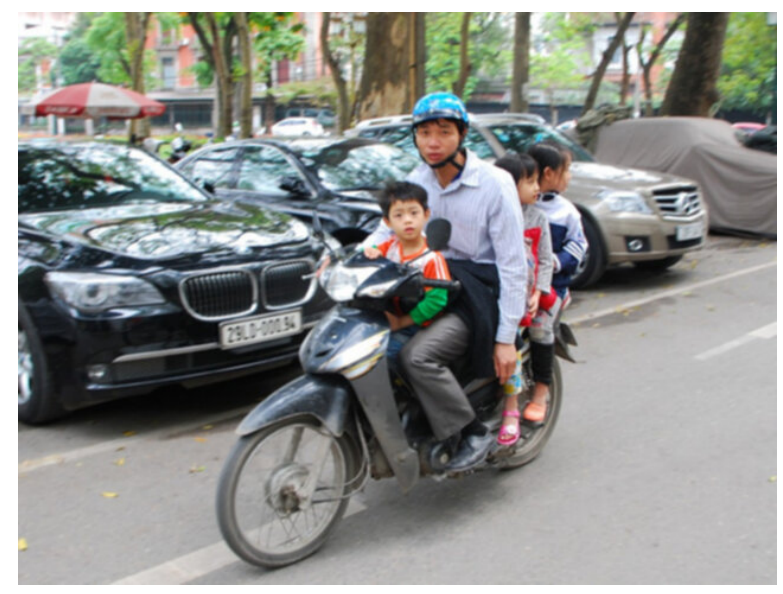

Question:is it sunny?

MRR candidate set

1. yes

2. yes, it is

3. yes it is sunny

4. it looks overcast, but i ca n't see the sky

5. it appears to be sunny

6. yes, its daytime and it looks sunny

7. no, it 's looks overcast

Top 10 from the remaining NDCG candidates

1. no it looks cloudy

2. does n't really seem to be

3. yes it is

4. no

5. it seems like it

6. looks like it is yes

7. appears to be

8. it 's overcast

9. not really

10. $\mathrm{i}$ believe it is

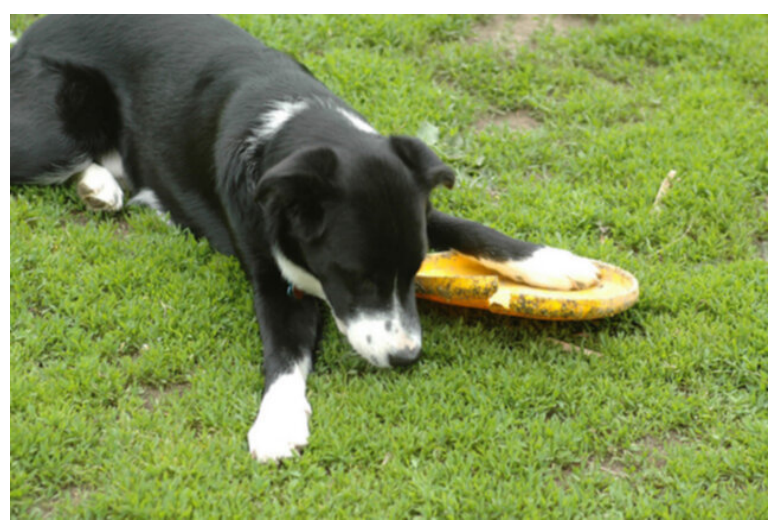

Question:do see any cars ?

MRR candidate set

1. no there are no cars

2. no cars

3. no

4. no, not at all 
Top 10 from the remaining NDCG candidates

1. nope

2. there is 0

3. 0 that $i$ can see

4. not that $\mathrm{i}$ can see

5. 0 visible

6. 0

7. no, ca n't see the street

8. not really

9. i do n't think so

10. no, she is not

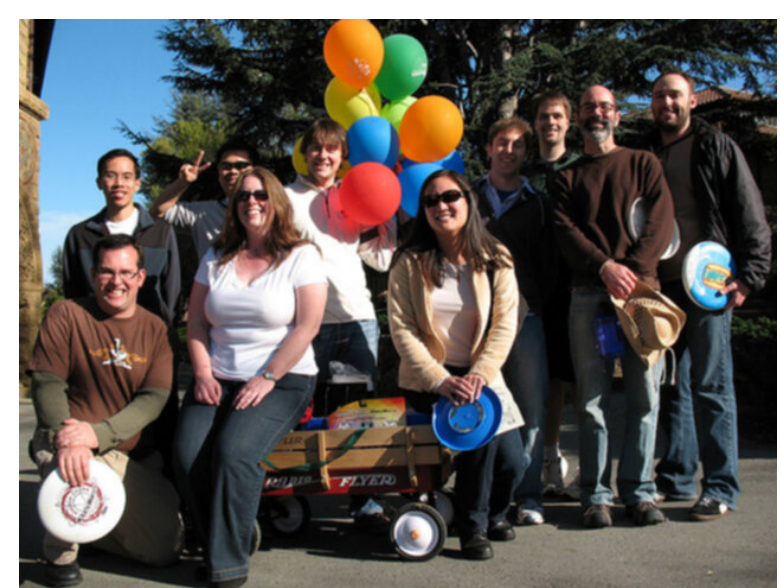

Question:are they outside?

\section{MRR candidate set}

1. yes

2. yes, they 're outside

3. yes, they are

4. yes they are

5. it looks like it

\section{Top 10 from the remaining NDCG candidates}

1. it seems like it

2. i think so

3. $\mathrm{i}$ believe so

4. yes, it is

5. yes it is outside

6. probably
7. looks like

8. yes, on a street corner

9. it looks outside

10. yes it is

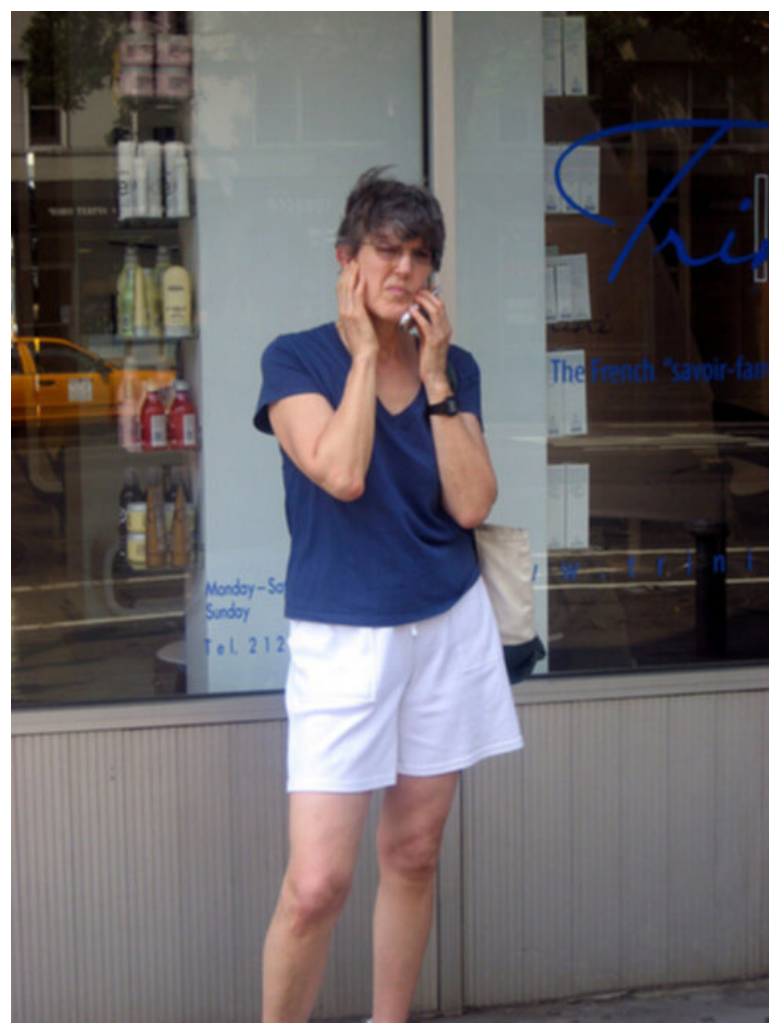

Question:what color are her shorts?

MRR candidate set

1. white

2. they are white

3. her pants are light blue

4. blue

5. salmon

Top 10 from the remaining NDCG candidates

1. looks like summer

2. looks blue

3. khaki

4. UNK bright blue

5. i think blue, hard to tell

6. mauve

7. grey 


\section{8. yes}

9. blue yellow and white

10. blue and black

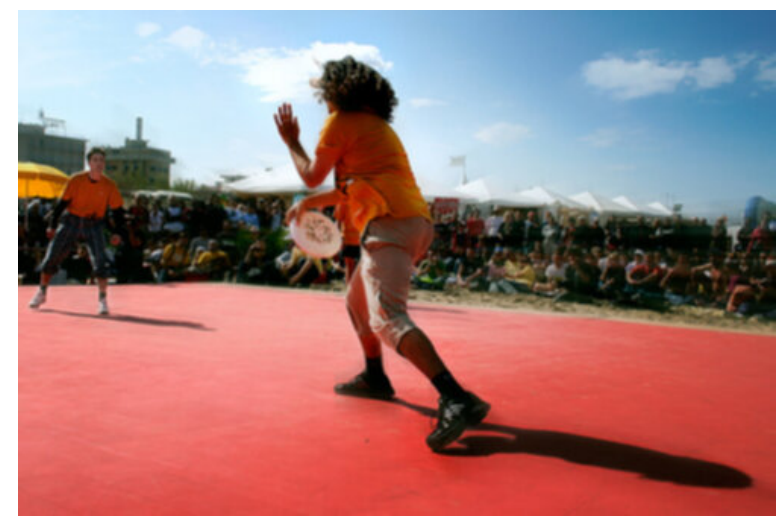

Question:is it sunny outdoors?

MRR candidate set

1. yes

2. yes it is

3. yes, it is

4. yes, it is a sunny day

5. yes sunny

\section{Top 10 from the remaining NDCG candidates}

1. it is

2. very

3. yes, the weather looks nice

4. yes the sun is shining

5. yes appears so

6. it appears to be sunny

7. it seems to be sunny

8. seems like it

9. yes, but i can not see the sun

10. yrd

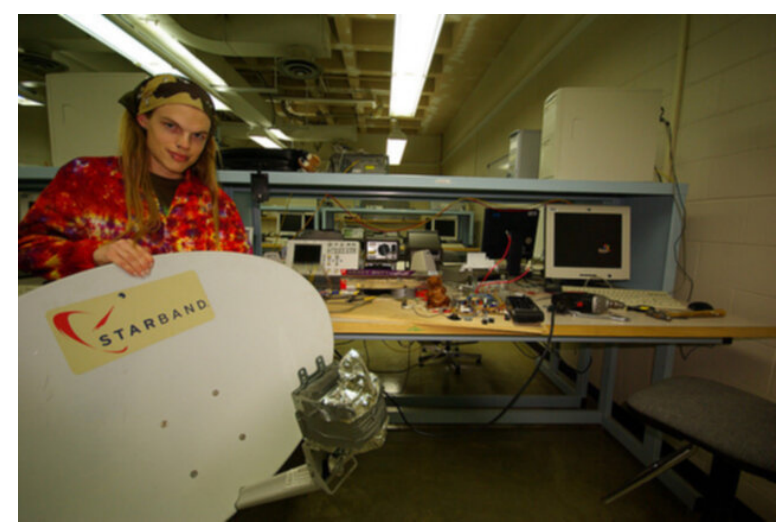

Question:do you see any lights ?

\section{MRR candidate set}

1. no

2. yes

3. yes i see 2 lights

4. i think so

5. yes many

\section{Top 10 from the remaining NDCG candidates}

1. 1 in the background

2. no i do n't

3. no, i do n't

4. nope

5. yes, 1 big 1

6. yes, some on the floor

7. i do n't think so

8. 'no i do n't

9. not that i can see

10. yes above the sink

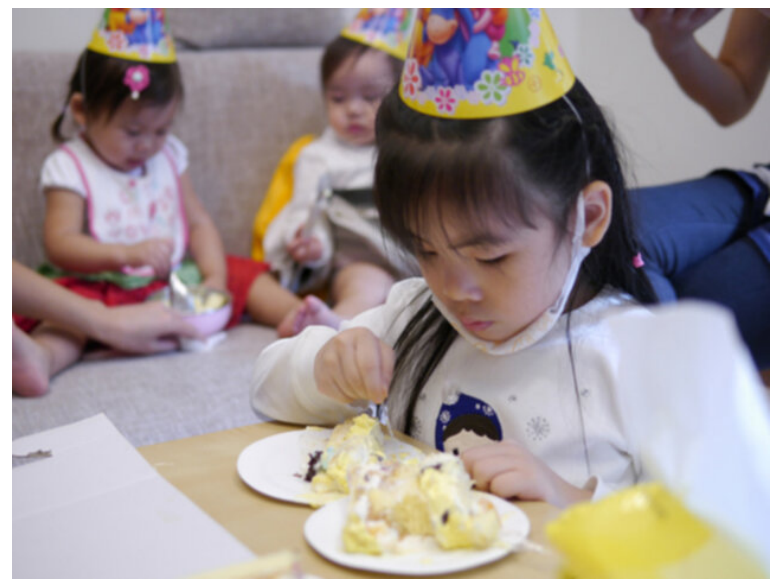

Question:are they outside?

MRR candidate set

1. no

2. yes

3. i do n't think so

4. i ca n't tell

5. nope 
Top 10 from the remaining NDCG candidates

1. i am not sure

2. ca n't tell

3. hard to tell

4. no, it 's indoors

5. i can not tell

6. i do n't think they are based on their clothing

7. not that $i$ can see

8. not sure

9. ca n't tell - maybe

10. i think so

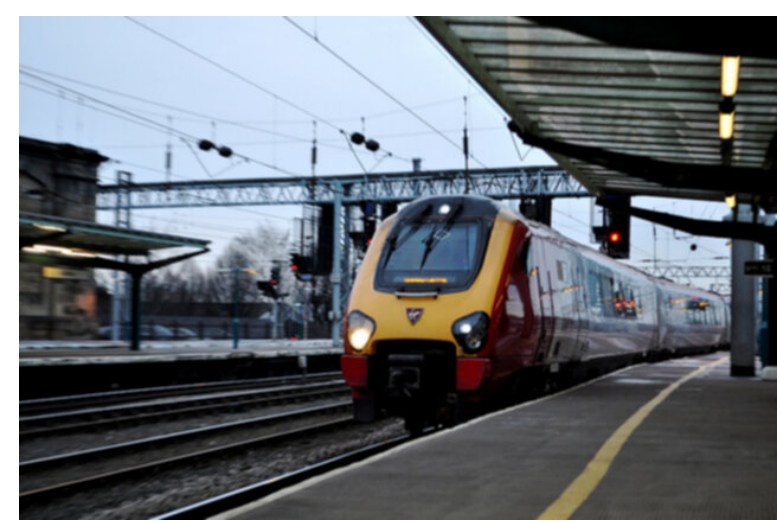

Question:is it a passenger train ?

MRR candidate set
1. yes
2. no
3. yes it is
4. yes, it is
5. yes, $i$ think so

\section{Top 10 from the remaining NDCG candidates}

1. yes, it looks like it

2. it appears to be

3. i think so

4. it looks like it, hard to tell

5. yes, it 's big

6. could be

7. i ca n't tell for sure, but i think so
8. maybe

9. UNK

10. 2

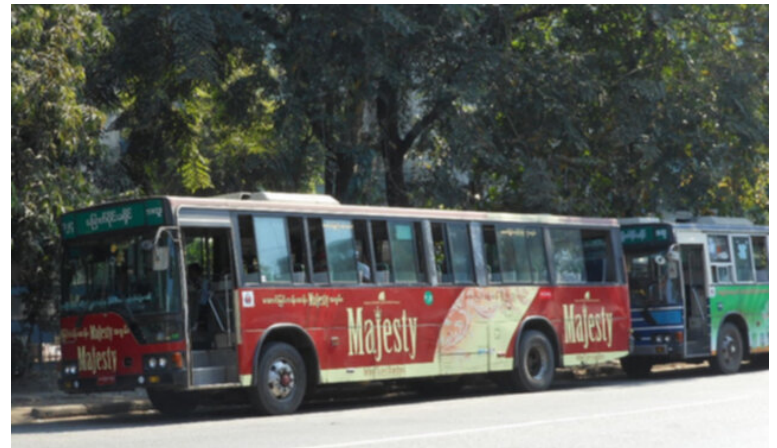

Question:is the bus doors open or closed? MRR candidate set
1. open
2. closed
3. i can not tell
4. i ca n’t tell
5. it 's open

\section{Top 10 from the remaining NDCG candidates}

1. ca n't tell

2. no doors

3. both are open

4. not sure

5. ca n't really tell

6. yes it is open

7. no

8. yes

9. no, closed

10. seems to be

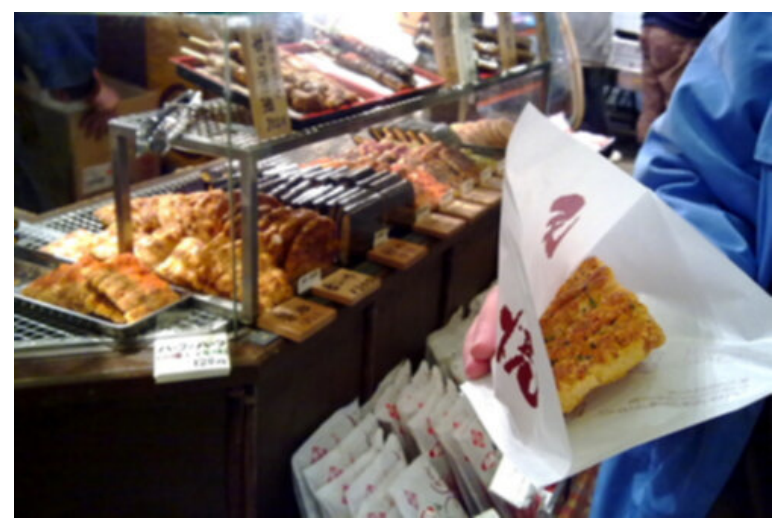


Question:describe the person holding the treat? MRR candidate set

1. ca n't see the person

2. i can only see their arm they are wearing blue

3. all $\mathrm{i}$ can see is a hand but probably a male

4. i can not tell

5. ca n't tell from the picture

6. i am unable to tell

\section{Top 10 from the remaining NDCG candidates}

1. i can not see

2. i ca n't tell

3. not visible not seen

4. i ca n't tell his back is towards me

5. ca n't tell

6. he looks confused

7. not sure

8. woman

9. UNK smiling

10. the shirt is striped in black and white

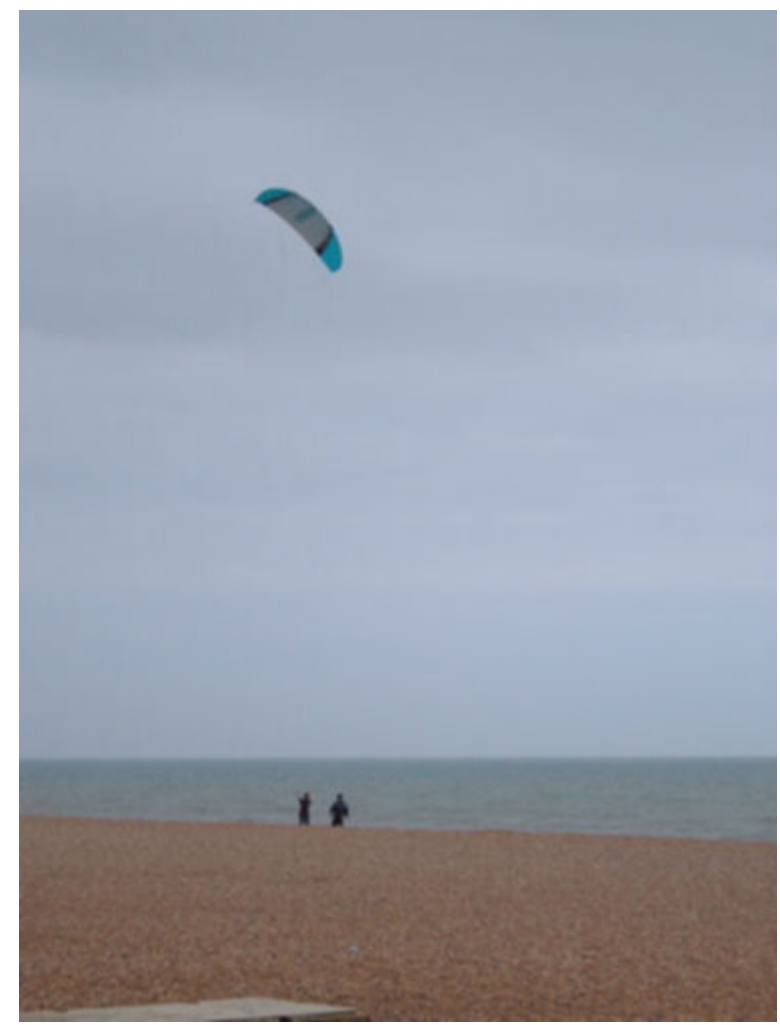

Question:how many people are there ?

MRR candidate set
1. 2

2. 3

3. there are 2

4. $\mathrm{i}$ think i see only 2

5. 2 in the picture

\section{Top 10 from the remaining NDCG candidates}

1. there are 3

2. at least 4

3. 4

4. i can see 4 or 5

5. there are tow

6. 1

7. more than 3

8. i can not tell

9. not sure

10. i ca n't tell

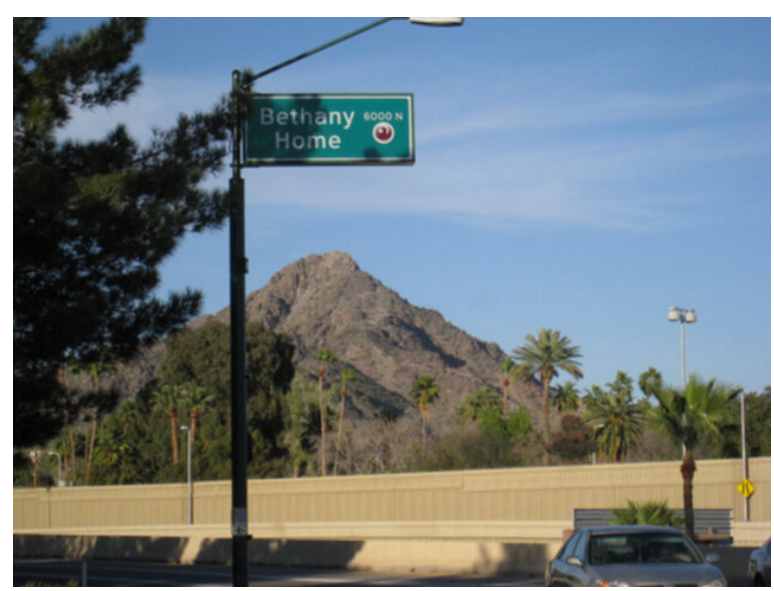

Question:is it day time?

MRR candidate set
1. yes
2. yes it is
3. yes, it is
4. it is
5. yep

\section{Top 10 from the remaining NDCG candidates}

1. yes' 
2. yes, it is day

3. yes, daytime

4. yes it is daytime

5. it is day time

6. yes it is , it 's sunny

7. yes, i believe so

8. it is daytime

9. i think so

10. it 's daytime

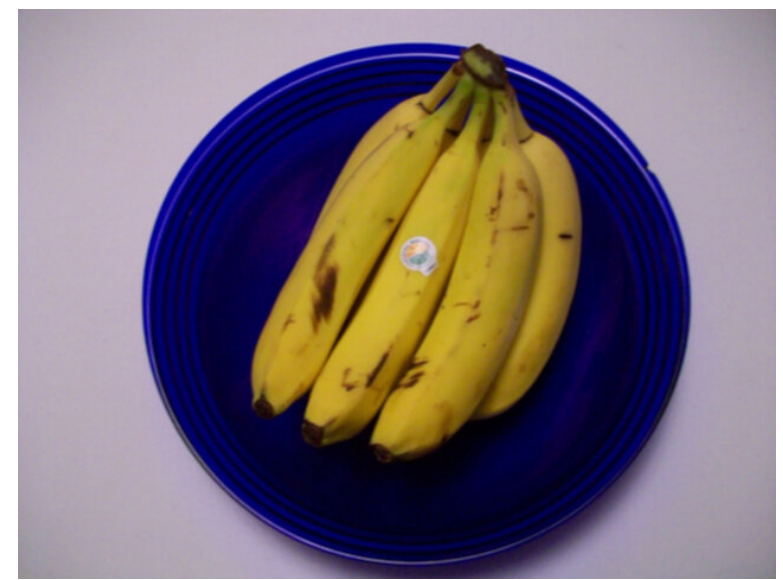

Question:are there any utensils on the table ? MRR candidate set

1. no

2. there are no utensils

3. nope

4. no, there are not

5. no, not that i can see

6. no there is not

Top 10 from the remaining NDCG candidates

1. no, there are 0

2. not that i can see

3. 0 that $i$ can see

4. 0 that can be seen

5. 0

6. 0 are visible

7. not seen
8. i do n't think so

9. not really that i can see

10. no other thing

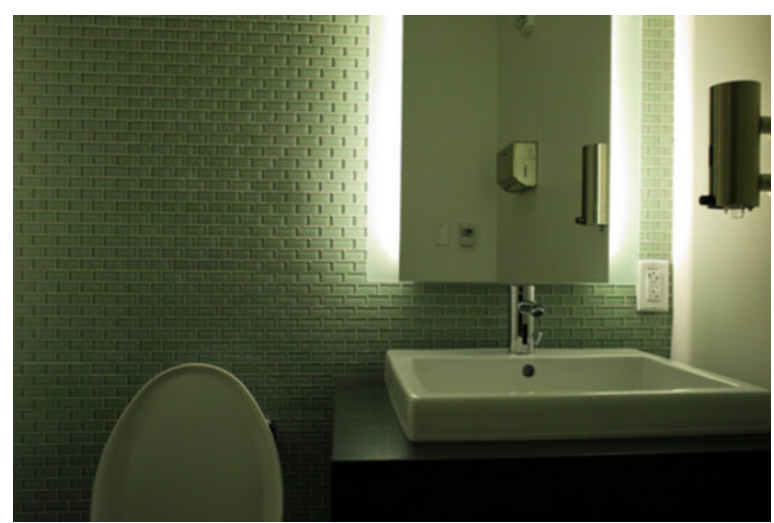

Question:can you see the floor?

MRR candidate set

1. no

2. yes

3. nope

4. i ca n't

5. no i ca n't

Top 10 from the remaining NDCG candidates

1. no i can not

2. no, the floor is n't visible

3. no, not at all

4. no you can not

5. not really

6. barely

7. i do n't think so

8. not that i can see

9. a little bit

10. yes, i can 


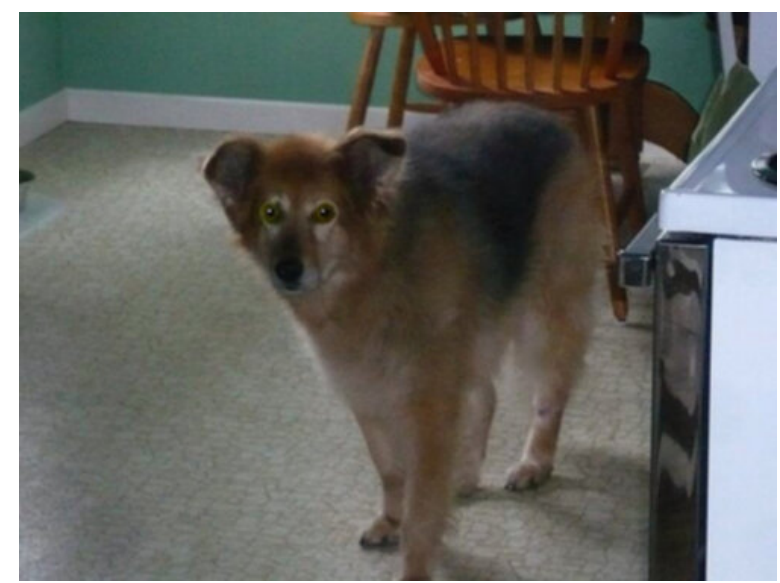

Question:what color is the dog?

\section{MRR candidate set}

1. brown

2. it is black and tan

3. it is mostly brown with some tan

4. brown and white

5. light brown

6. brown with white

\section{Top 10 from the remaining NDCG candidates}

1. $\tan$ color

2. it is reddish-brown

3. brown and black

4. brown, black, $\tan$, and white

5. black and brown

6. i think its a golden retriever, so light yellow

7. browns and blacks

8. white and brown

9. no people

10. UNK

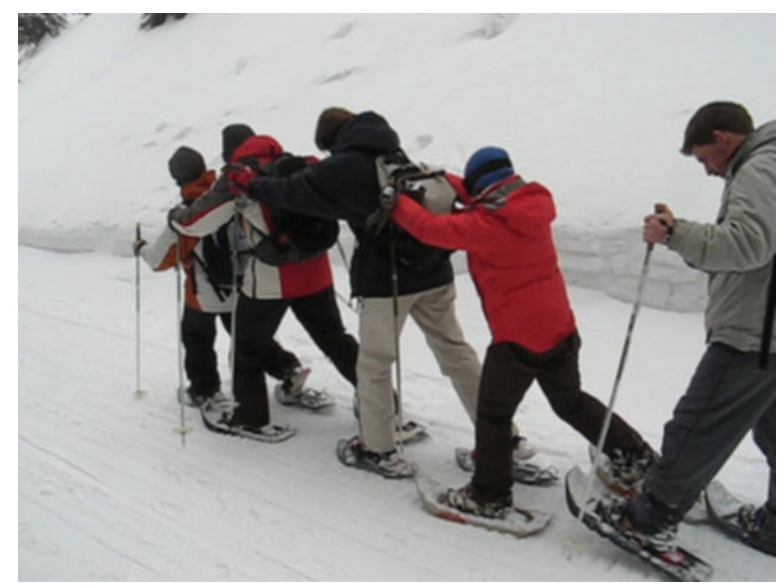

Question:are they wearing ski goggles?

MRR candidate set

1. i can not tell

2. no

3. i just see there backs i ca n't see their faces

4. yes

5. i ca n't tell

6. no, they are n't wearing goggles

7. ca n't tell

Top 10 from the remaining NDCG candidates

1. ca n't see faces see backs

2. i am not sure

3. no they are not

4. $\mathrm{i}$ believe so

5. yes they are

6. nope

7. not that i can see

8. i do n't think so

9. again, i can not see their face

10. they look like it



Question:do the trees have leaves?

MRR candidate set

1. yes

2. some do

3. yes, they are

4. yes, i think so 
Top 10 from the remaining NDCG candidates

1. yes very fresh looking

2. the $1 \mathrm{i}$ can see does, yes

3. long

4. i think so

5. at least 2 of them do

6. i 'm sure it can

7. yes, it is

8. yes there 's clearance

9. mostly brown color

10. maybe

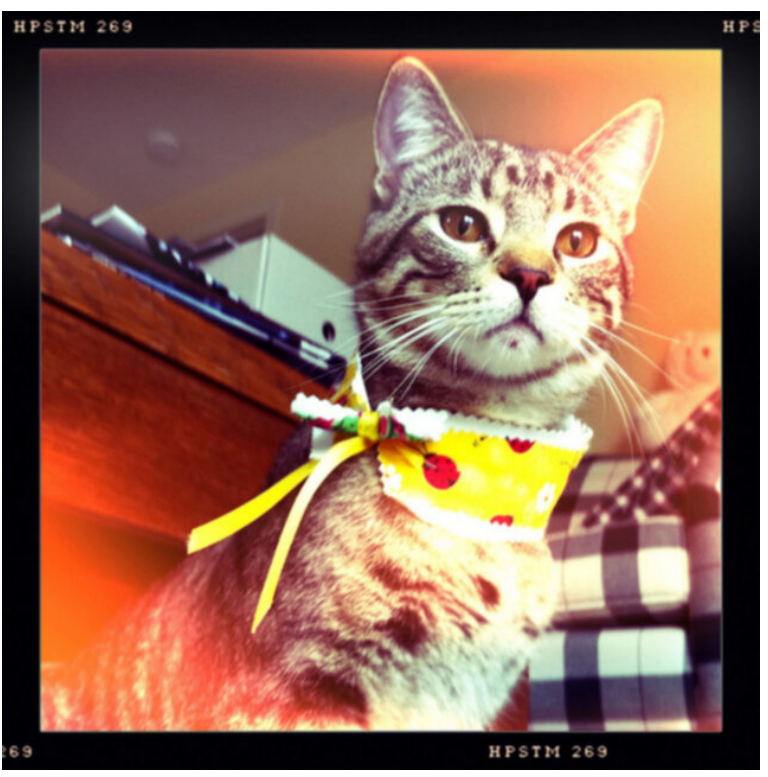

Question:how big is the table?

MRR candidate set

1. i can not tell

2. i ca n't tell

3. hard to say, it 's too close up

4. ca n't tell

5. close up ca n't tell

\section{Top 10 from the remaining NDCG candidates}

1. ca n't tell from the picture

2. looks pretty big to me

3. pretty small
4. not sure

5. pretty large

6. quite large

7. medium size

8. medium sized

9. big

10. it 's more narrow than most

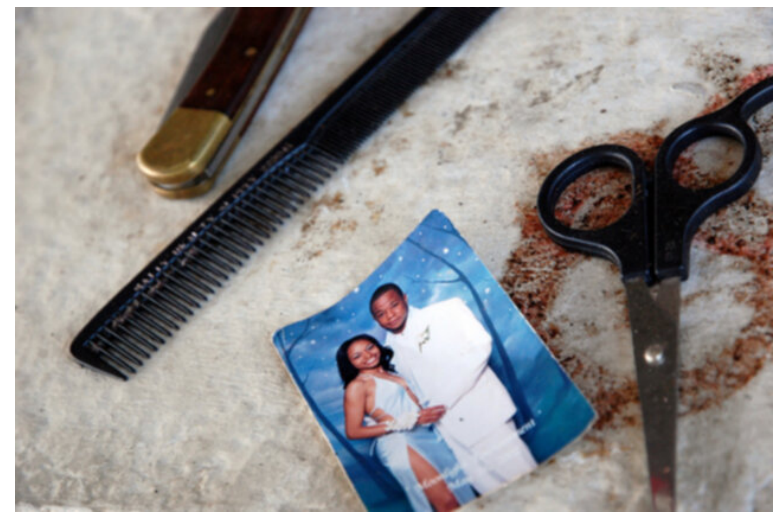

Question:what kind of scissors?

MRR candidate set

1. hair cutting scissors

2. silver

3. i can not tell

4. i ca n't tell

5. not sure

Top 10 from the remaining NDCG candidates

1. black

2. ca n't tell

3. larger

4. wood

5. grey

6. gray and white

7. bear

8. blue

9. brown

10. ca n't tell, too close 


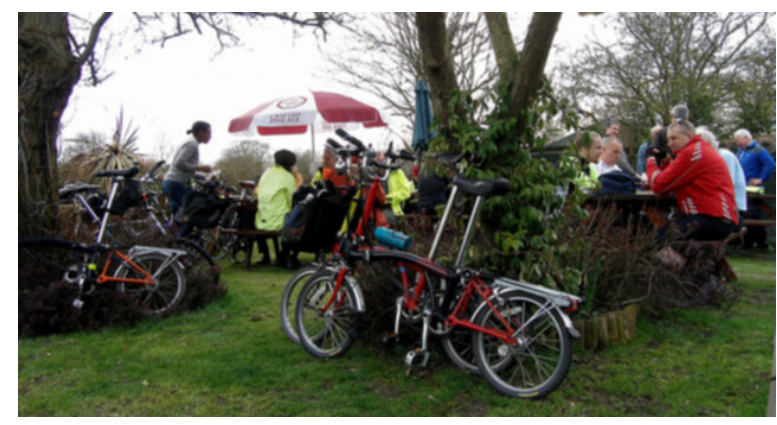

Question:any signs?

MRR candidate set

1. no

2. yes

3. 0

4. not that i can see

5. nope

6. not that i see

Top 10 from the remaining NDCG candidates

1. 0 visible

2. not visible

3. nno

4. yes a couple

5. i do n't think so

6. yes a few

7. yes, 1

8. 1

9. not really

10. ca n't see

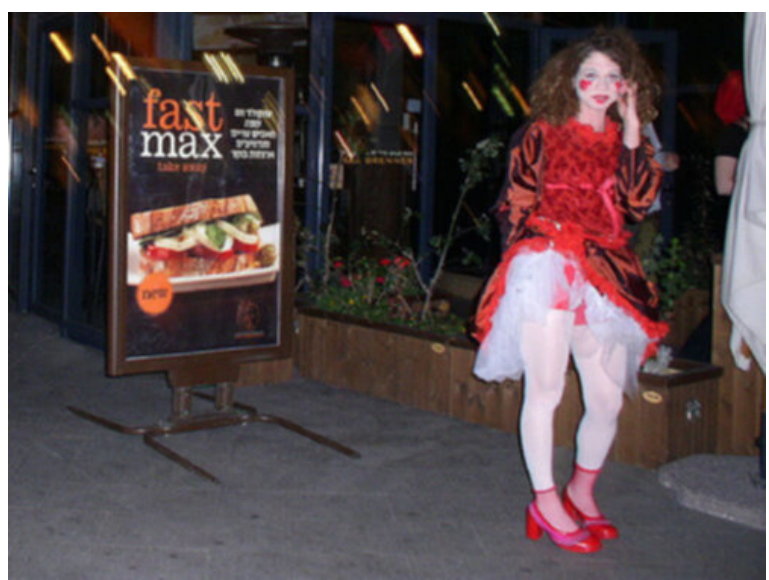

Question:is it american?

MRR candidate set
1. do n't know

2. no

3. yes

4. ca n't tell

5. i ca n’t tell

6. can not tell

7. i can not tell

Top 10 from the remaining NDCG candidates

1. not sure

2. i do n't think so

3. nope

4. i think so, i ca n't tell

5. maybe

6. no it is n't

7. i believe so, but ca n't say for sure

8. i think so

9. perhaps

10. it appears to be but it is hard to confirm

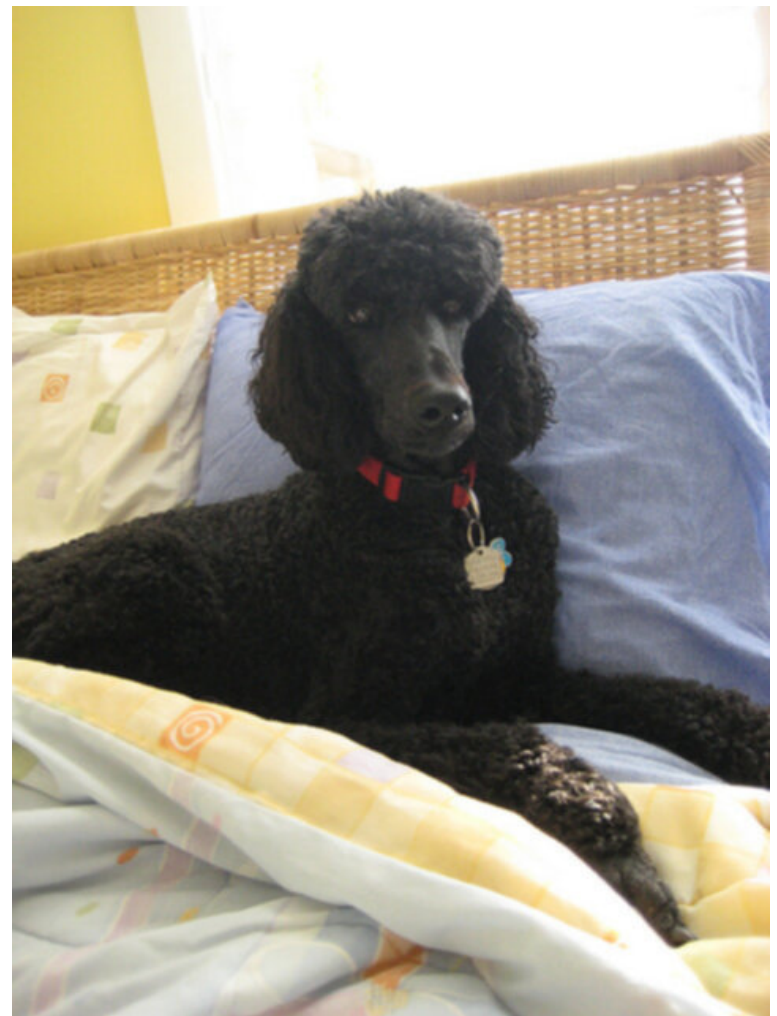

Question:is the bed outside?

MRR candidate set 
1. no

2. i can not tell

3. no, it does n't appear to be

4. no it is not

5. no it 's not

6. nope

\section{Top 10 from the remaining NDCG candidates}

1. do n't think so

2. i do n't think so

3. does n't look like it

4. don' t see a bed

5. no, it is inside

6. not that i can see

7. inside

8. i ca n't tell

9. i do n't think so, it 's hard to tell

10. it is not from what i see

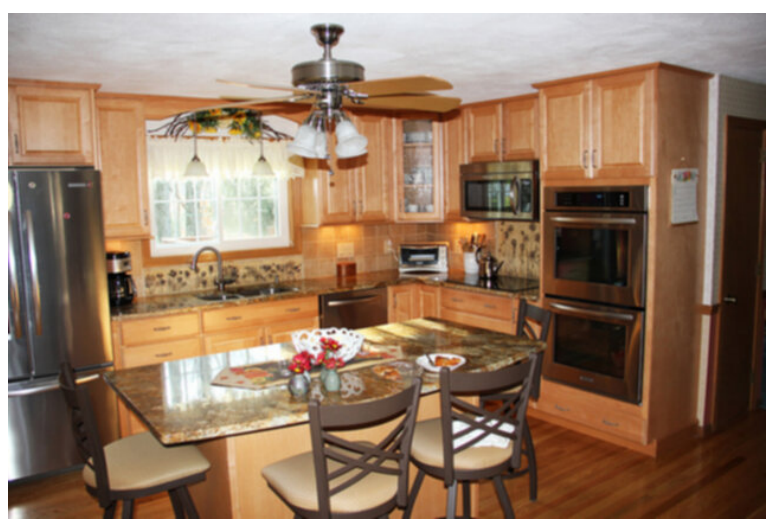

Question:is there windows?

\section{MRR candidate set}

1. yes there is

2. yes

3. a few

4. there is a window which has bright sunlight streaming in

5. yes, it looks like

Top 10 from the remaining NDCG candidates
1. there are about 3

2. yes, it is

3. i think so

4. yes there are tree's

5. yes it is

6. only 2 are

7. yes, there are some storefronts

8. in the distance

9. 1

10. nope

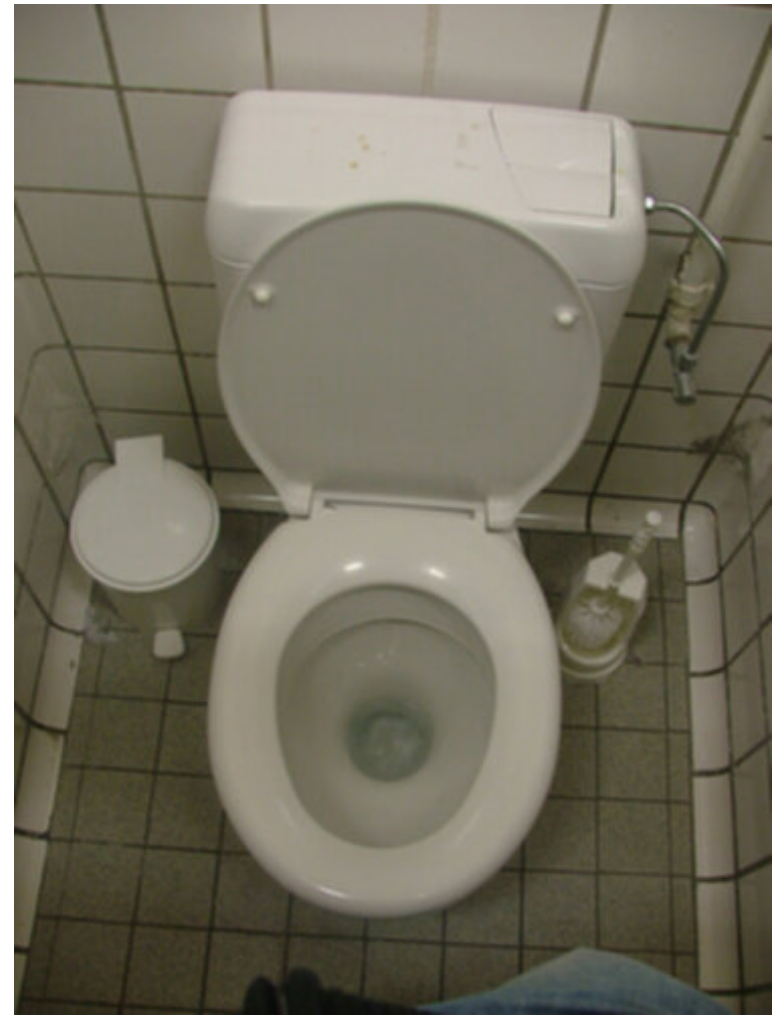

Question:is there a sink ?

MRR candidate set

1. no $\sin \mathrm{k}$

2. not that i can see

3. not in the image

4. nope

5. i ca n't see 1

6. there is not

Top 10 from the remaining NDCG candidates 
1. no there is n't

2. no

3. not that i see

4. not seen in the image

5. yes, there appears to be one, but i can not actually see it

6. not visible

7. ca n't see it in the picture

8. yes there is a faucet no actual sink

9. ca n't see 1

10. yes there is

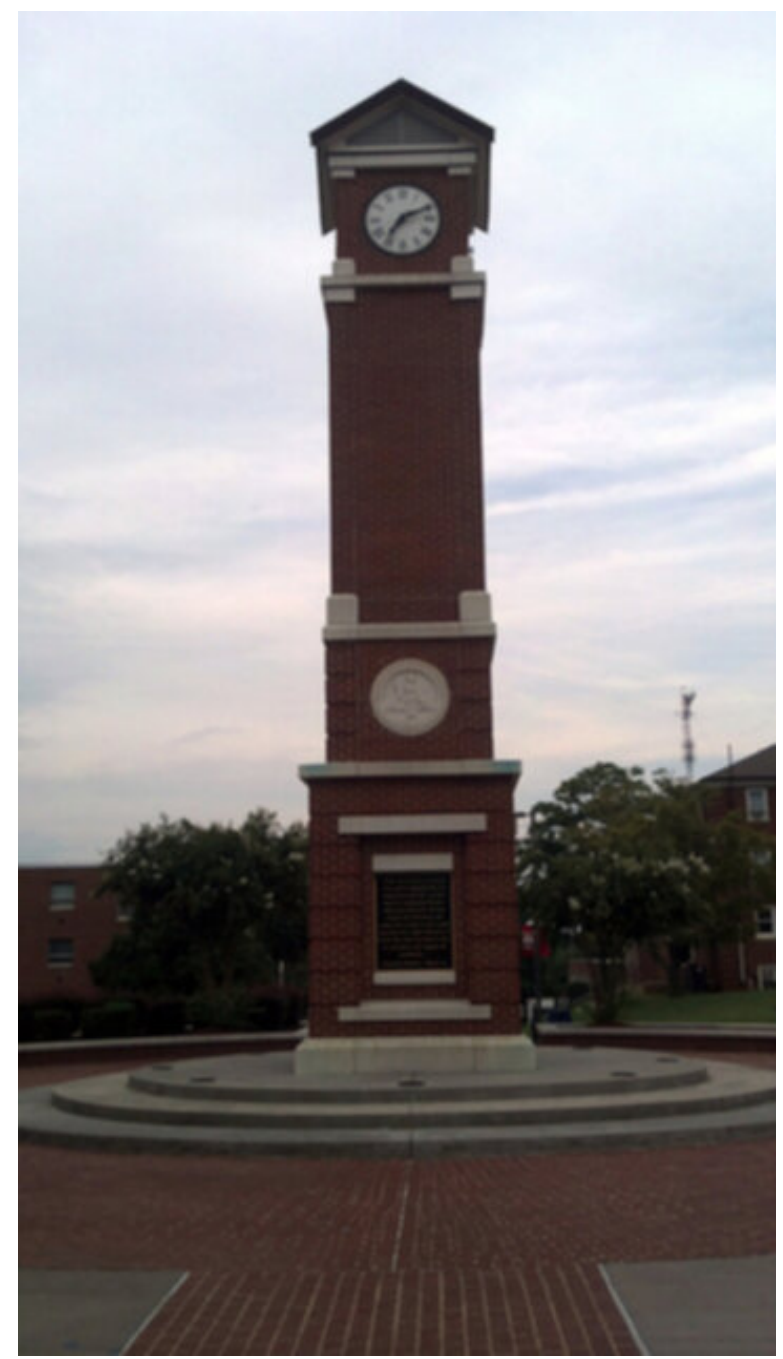

Question:is the clock tower large?

MRR candidate set

1. yes

2. yes, huge
3. yes it is

4. yes, it is

Top 10 from the remaining NDCG candidates

1. yes it 's pretty big

2. not really

3. i think so

4. kinda

5. medium size

6. no

7. normal size

8. medium sized

9. medium

10. it 's wide but not tall

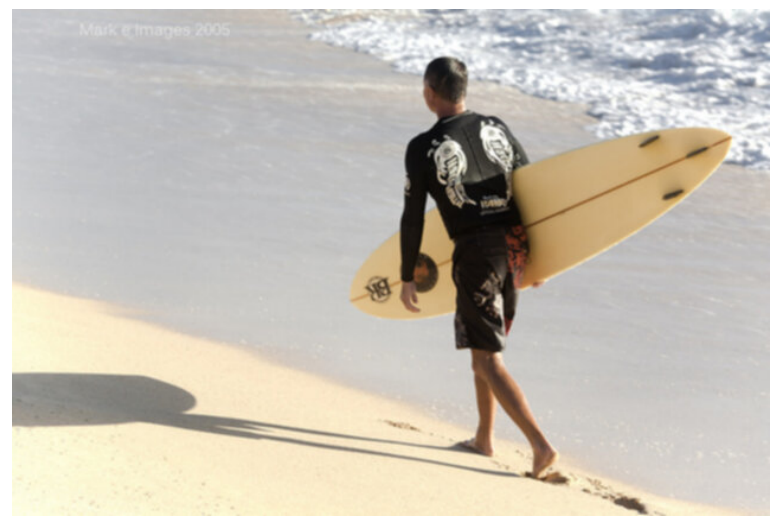

Question:can you see the water? MRR candidate set

1. yes

2. no

3. yes i can

4. yes, i can

Top 10 from the remaining NDCG candidates

1. yews

2. i think so

3. yes a little bit

4. yes it is blue

5. a little bit

6. partially 
7. looks to be

8. barely

9. a bit

10. yes, it is

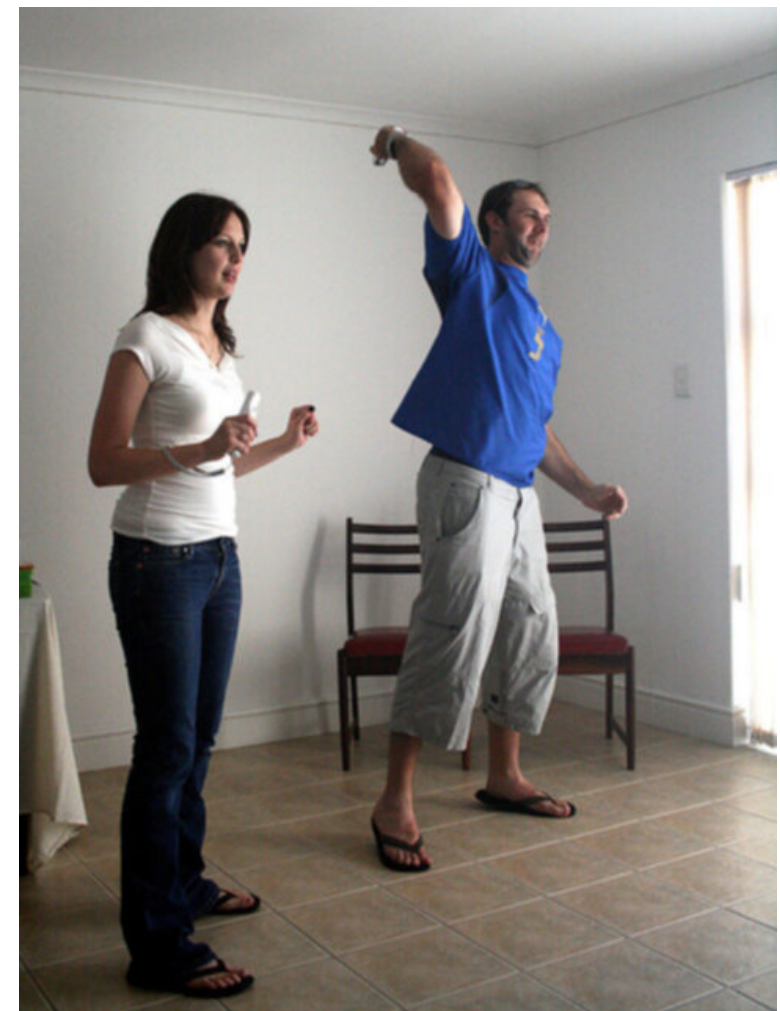

Question:are the man and woman the only people in the photo?

MRR candidate set

1. yes

2. no

3. no, they are not

4. yes both are

5. nope

\section{Top 10 from the remaining NDCG candidates}

1. i think so

2. no other people that $\mathrm{i}$ can see

3. yes that is all

4. i do n't think so

5. just the woman and the little girl

6. yes, it is

7. yes it is
8. not that i can see

9. yes it 's look like it was take late at night

10. not really

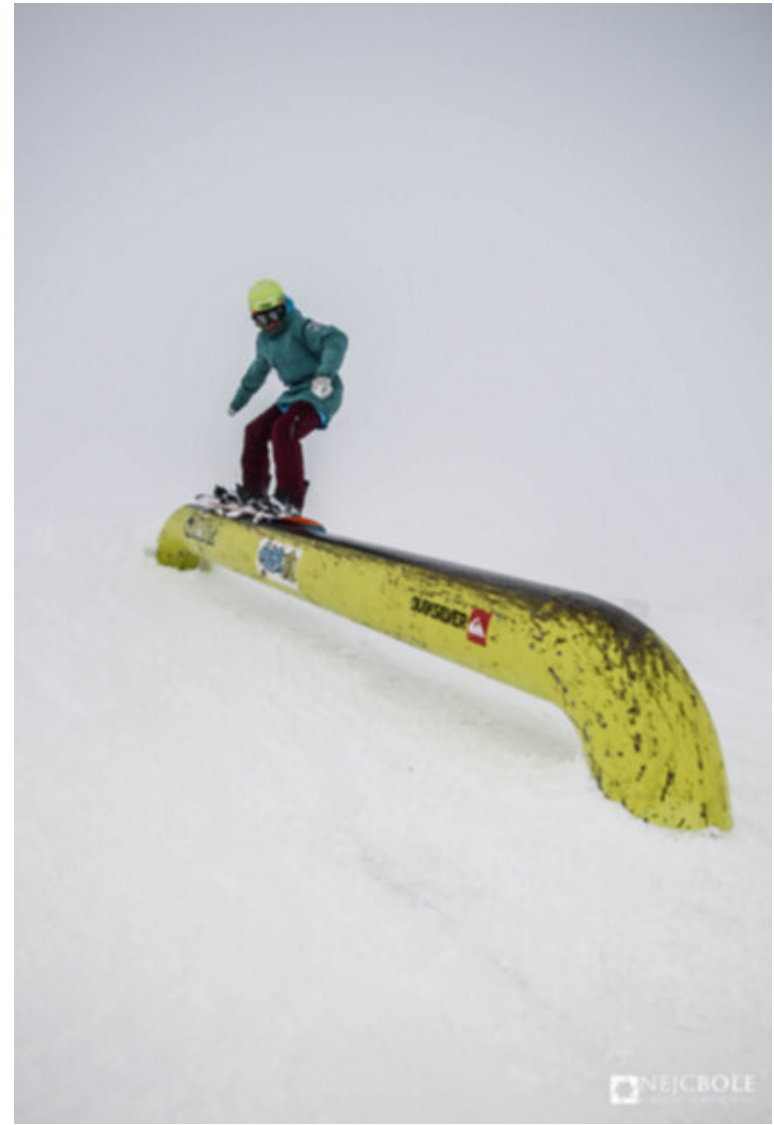

Question:is anyone watching him ?

MRR candidate set

1. no

2. i do not see anyone else in the picture

3. not that $i$ can see

4. i ca n't tell

5. nope

\section{Top 10 from the remaining NDCG candidates}

1. i do n't think so

2. i can not tell

3. ca n't tell

4. no he 's not

5. hard to see

6. no he is not

7. not sure 

8. can
9. possibly

10. i believe so, but ca n't say for sure

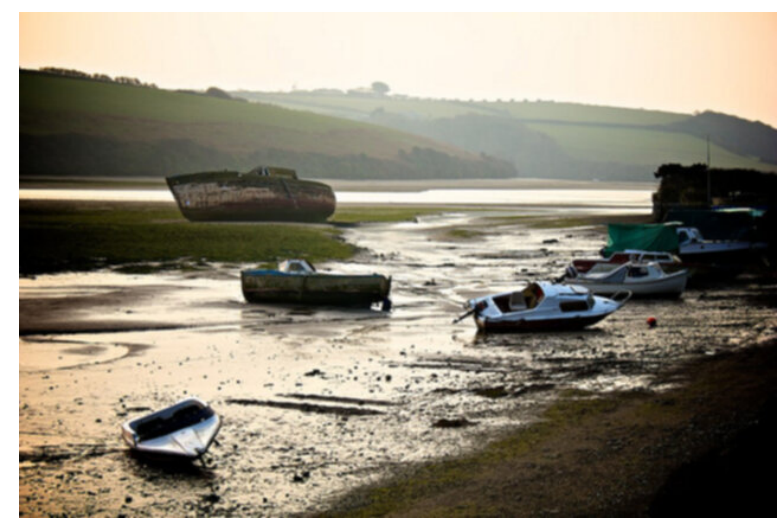

Question:are they big boats or small boats?

MRR candidate set

1. small

2. they look small

3. they are all different sizes

4. to scale they are large, medium and small UNK

5. medium

\section{Top 10 from the remaining NDCG candidates}

1. $\operatorname{mix}$

2. both

3. on the small side

4. no, more like small yachts

5. they look older

6. no, there are 2 larger ones and 1 smaller 1

7. no the boats are all on the sand

8. no they are small

9. no about medium like the kind that cut hair

10. yes

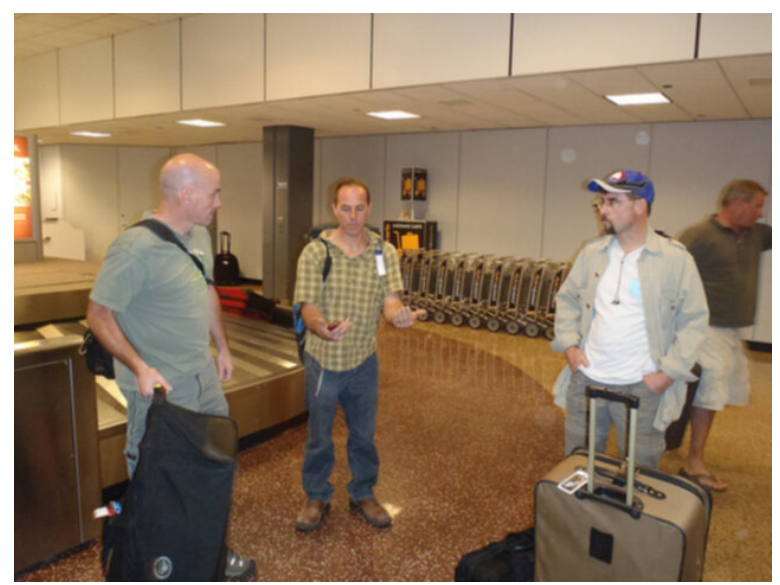

Question:are there other people there?

MRR candidate set

1. no

2. yes

3. yes there are

\section{Top 10 from the remaining NDCG candidates}

1. yes a few others

2. yes, there are

3. not that i can see

4. yeah, 3 total

5. not in the photo

6. nope

7. yes, there are 2 other people in the background

8. a couple in the background, and 1 woman barely seen in the foreground

9. yes a lot

10. 3 people

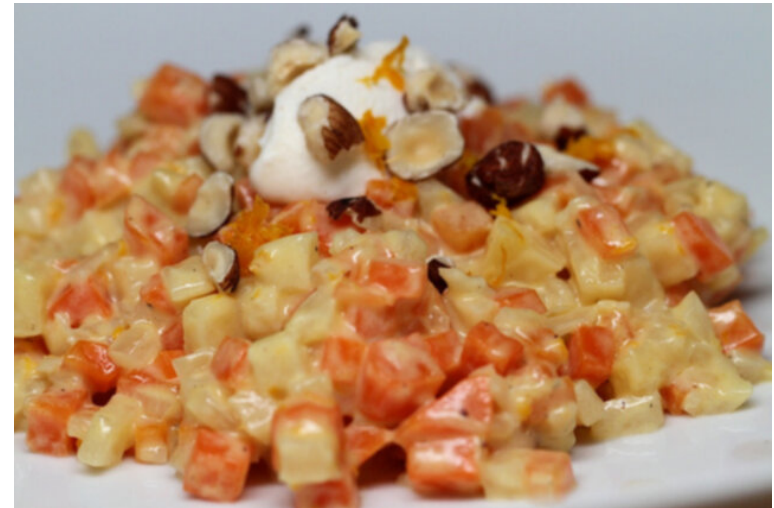

Question:what is there the most of ? MRR candidate set 
1. the carrots and what looks like maybe corn are about equal

2. i can not tell

3. i ca n't tell

4. i ca n't see the image

5. not sure

\section{Top 10 from the remaining NDCG candidates}

1. meat

2. ca n't tell

3. it looks quite fresh

4. it looks like it might be apple

5. it appears to be people but i do n't have the best view

6. looks brown or black spots on cream

7. light colored

8. a little bit

9. hard to tell, tomato sandwich

10. just the ocean

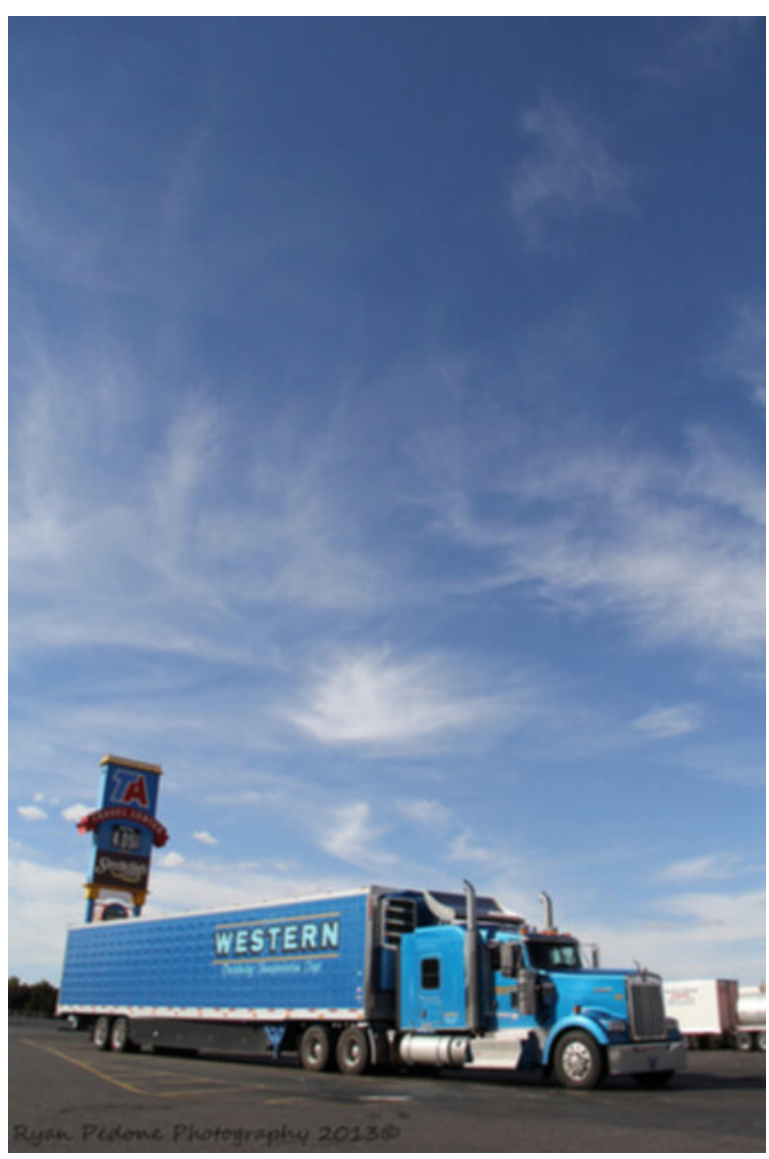

Question:does the field have a lot of grass ? MRR candidate set

1. no

2. it is in a parking lot

3. no, it does n't

4. nope

5. not that i can see

Top 10 from the remaining NDCG candidates

1. that part is not in the photo

2. no not in view

3. not really

4. not especially

5. 0

6. ca n't tell

7. i do n't think so

8. i can not tell

9. i ca n't tell

10. grass

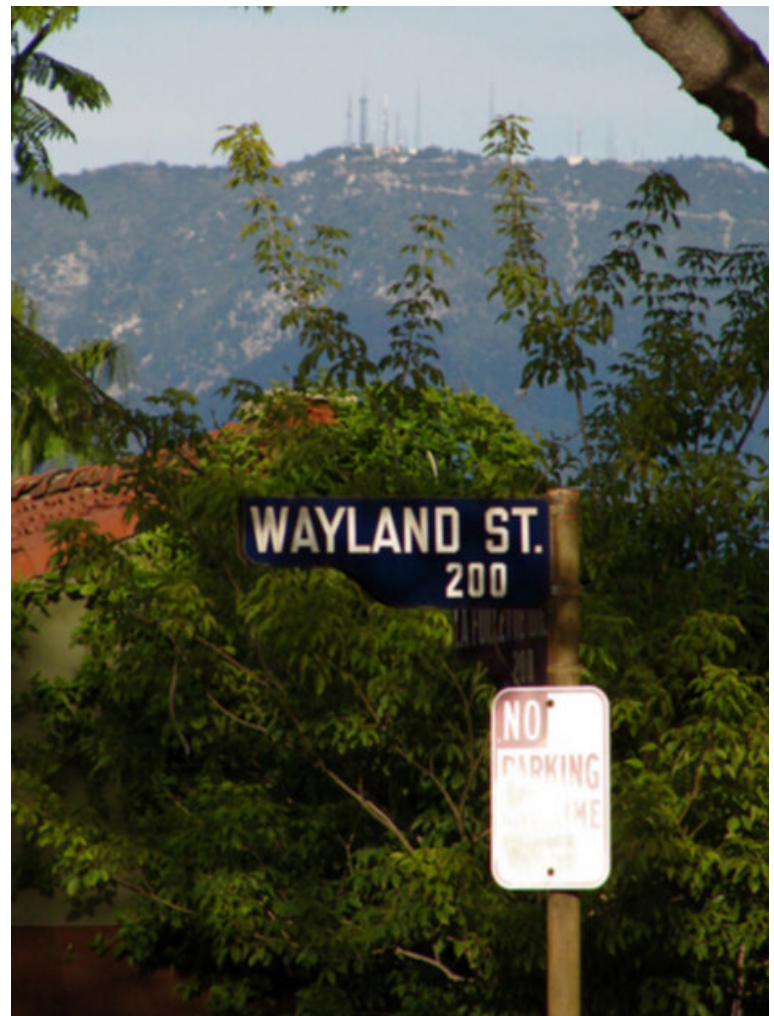

Question:what color is the sign? MRR candidate set 
1. white

2. there is a blue sign and a white with red writing sign

3. blue

4. black and white

5. white and black

\section{Top 10 from the remaining NDCG candidates}

1. white with writing

2. it is white

3. white, black and red

4. the sign is black

5. yes, it is

6. red and white

7. they are white with black around the edges

8. 1 yellow and 1 white

9. yes it is

10. UNK

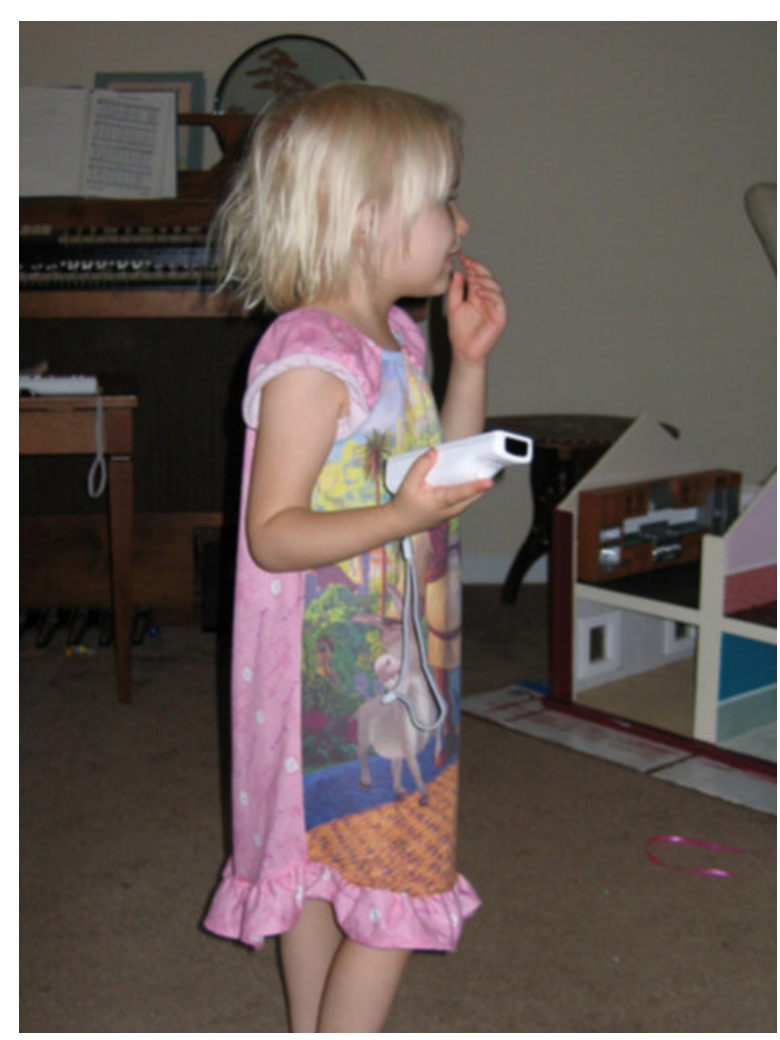

Question:how old does she look?

MRR candidate set

1. 2
2. 3

3. about 9 , i am not sure

4. maybe 2 at oldest

5. maybe under 1 year old

6. maybe 5

\section{Top 10 from the remaining NDCG candidates}

1. maybe 7

2. 4

3. 1

4. maybe $7-10$

5. looks like a preteen girl

6. $7-9$

7. 5

8. not sure

9. 7 or 8

10. around 8

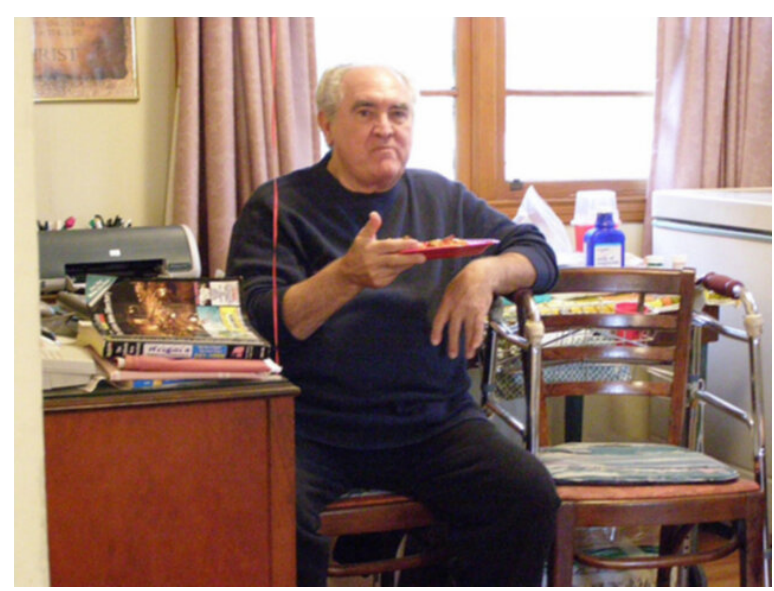

Question:is there a computer on the desk ? MRR candidate set

1. no

2. yes

3. i think so

4. looks like it

\section{Top 10 from the remaining NDCG candidates}

1. yes, it is

2. not that i can see 
3. yes but it is not on

4. yes, 1

5. yeds

6. nope

7. not seen

8. no, there is n't

9. the top of the desk is not visible

10. no there is n't

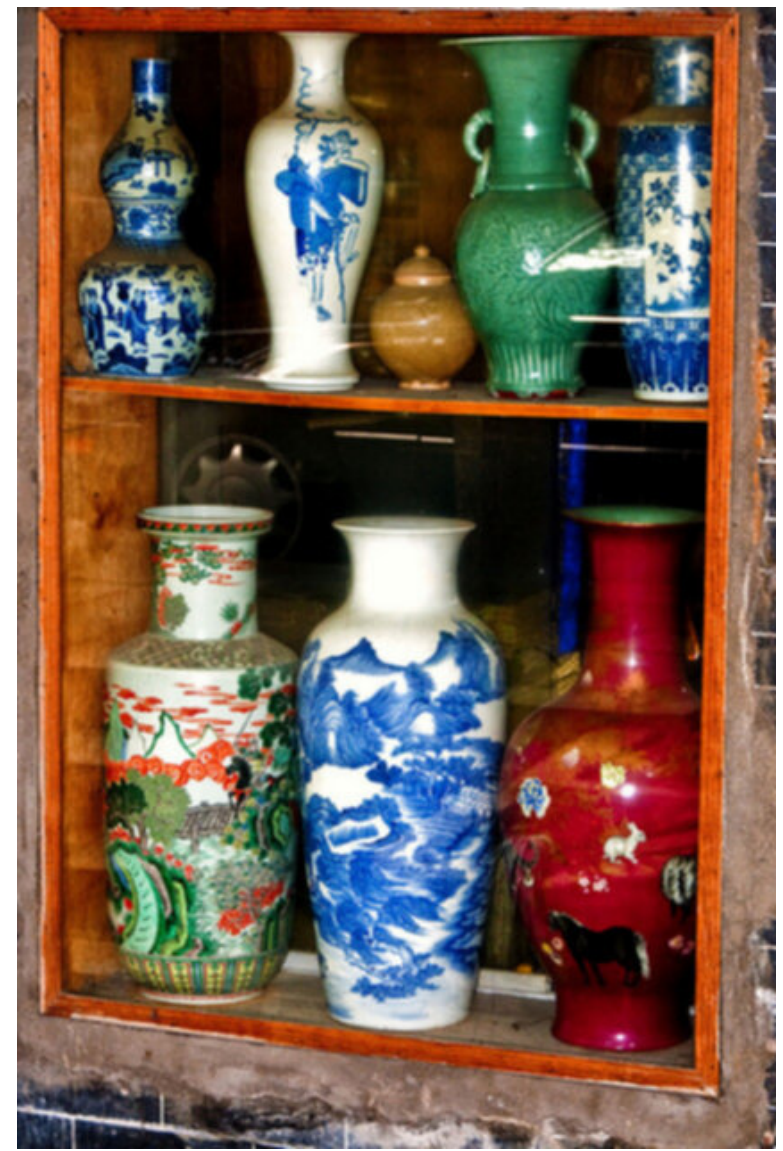

Question:are there people?

MRR candidate set

1. no

2. no people

3. nope

4. no there are no people

5. no there are n't

Top 10 from the remaining NDCG candidates

1. no people are visible

2. not that i can see
3. no people at all

4. i do n't see any

5. 0 that $i$ can see

6. 0

7. not that can be seen

8. nobody in the photo

9. no, this is a looking up picture

10. i do n't think so

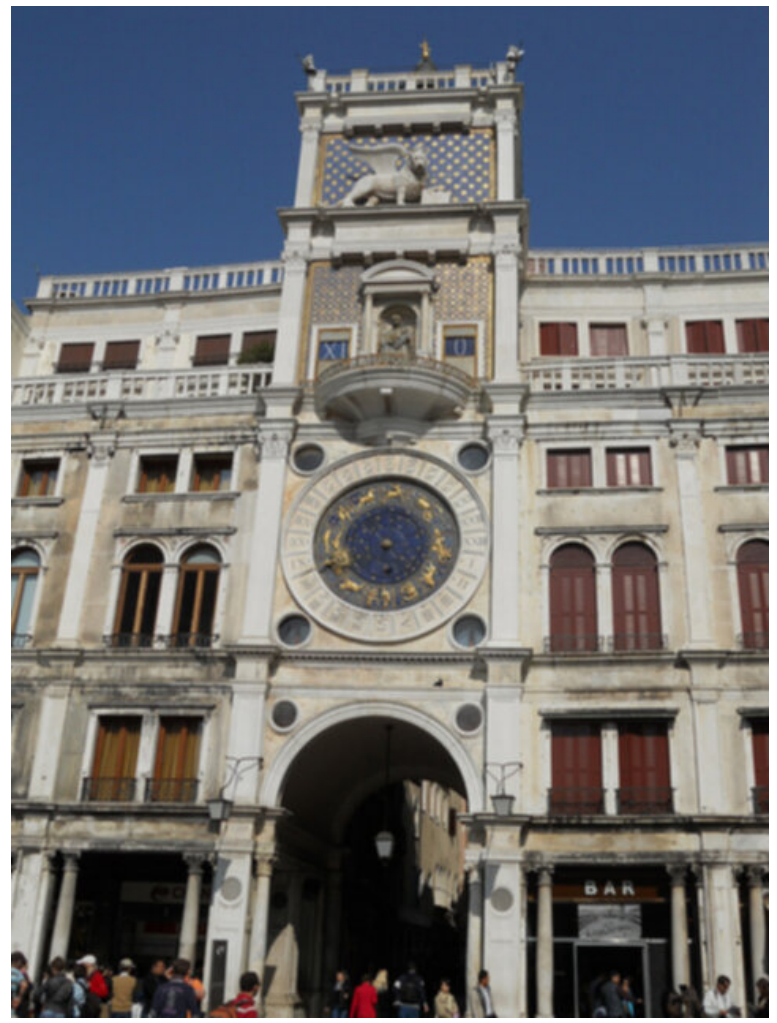

Question:how many stories is it?

MRR candidate set

1. ca n't tell

2. i can not tell

3. ca n't tell because the photo is cut but at least 3

4. i ca n't tell

5. maybe 3

6. not sure

Top 10 from the remaining NDCG candidates

1. 3

2. i think 4 
3. at least 4

4. ca n't see the bottom part

5. 2

6. at least 5

7. 4

8. 5 that $i$ can see

9. i see 4

10. there are only 2

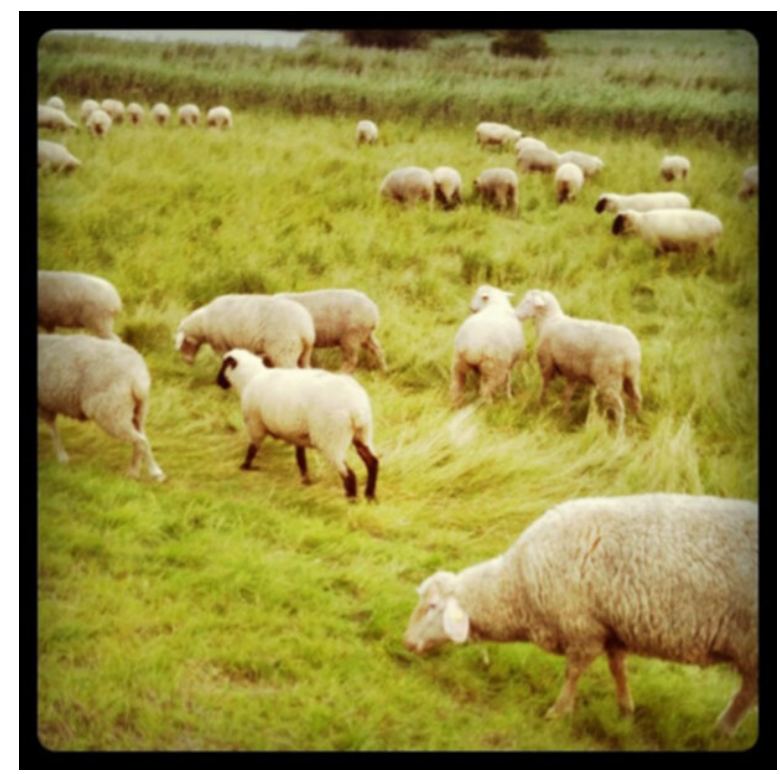

Question:is the grass green?

MRR candidate set

1. yes

2. yes, some is

3. some of it yes

4. yes it is

5. yes, it is

Top 10 from the remaining NDCG candidates

1. there is, yes

2. some

3. yes, i think so

4. some parts of it

5. light greenish yellow

6. lots of grass but yellow
7. i think so

8. green

9. yes, they are

10. yes light brown

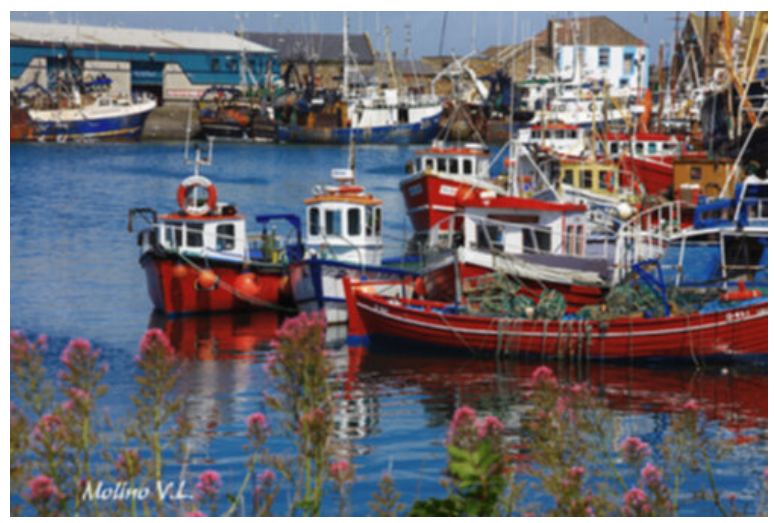

Question:anything else interesting about the photo ?

\section{MRR candidate set}

1. the colors are very vibrant

2. no

3. not really

4. nope

Top 10 from the remaining NDCG candidates

1. yes

2. not that $i$ can see

3. no, there are n't

4. i do n't think so

5. looks great

6. just the sea doo

7. 0

8. no there are n't

9. not visibly, but i 'd say probably so

10. yes, it is 


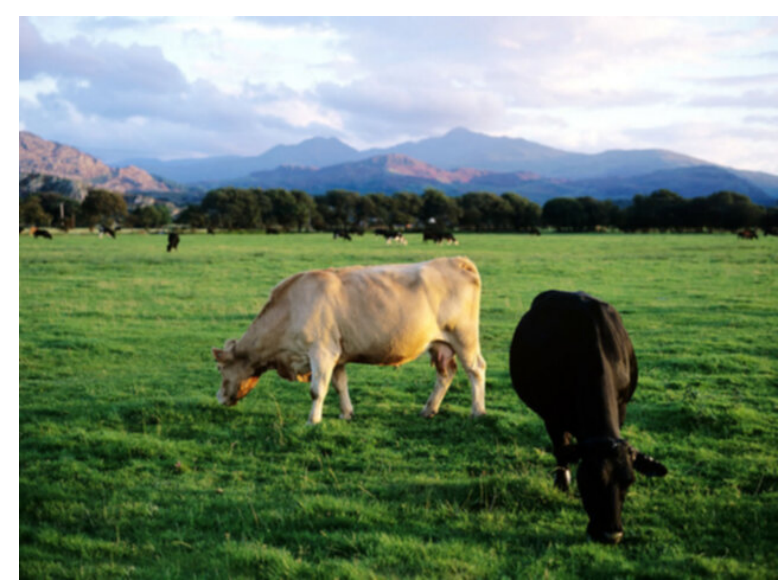

Question:are there any farmers near them ? MRR candidate set

1. there are no people visible

2. no, there are n't any people in the image

3. no, there is not

4. no there are not

5. no there are n't

6. nope

\section{Top 10 from the remaining NDCG candidates}

1. no

2. not that $i$ can see

3. there are no people in the image

4. no there isn' ' $t$

5. no, there are no people

6. i do n't see any

7. no there 's not

8. there are no people

9. no people

10. 0 i can see

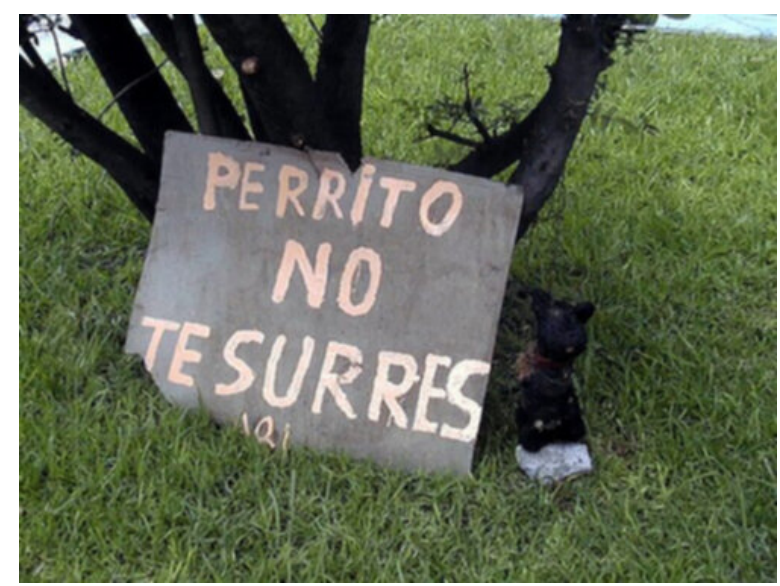

Question:what does the sign say?

MRR candidate set

1. welcome to UNK

2. UNK no UNK

3. it just says UNK dame with an arrow pointing left

4. words

5. UNK UNK

6. s gay st

\section{Top 10 from the remaining NDCG candidates}

1. join our csa

2. beautiful code : leading UNK explain how they think

3. the UNK of heaven

4. the original tour

5. twin peaks with 200 sign on top

6. number 9

7. not sure

8. UNK

9. no

10. alaska

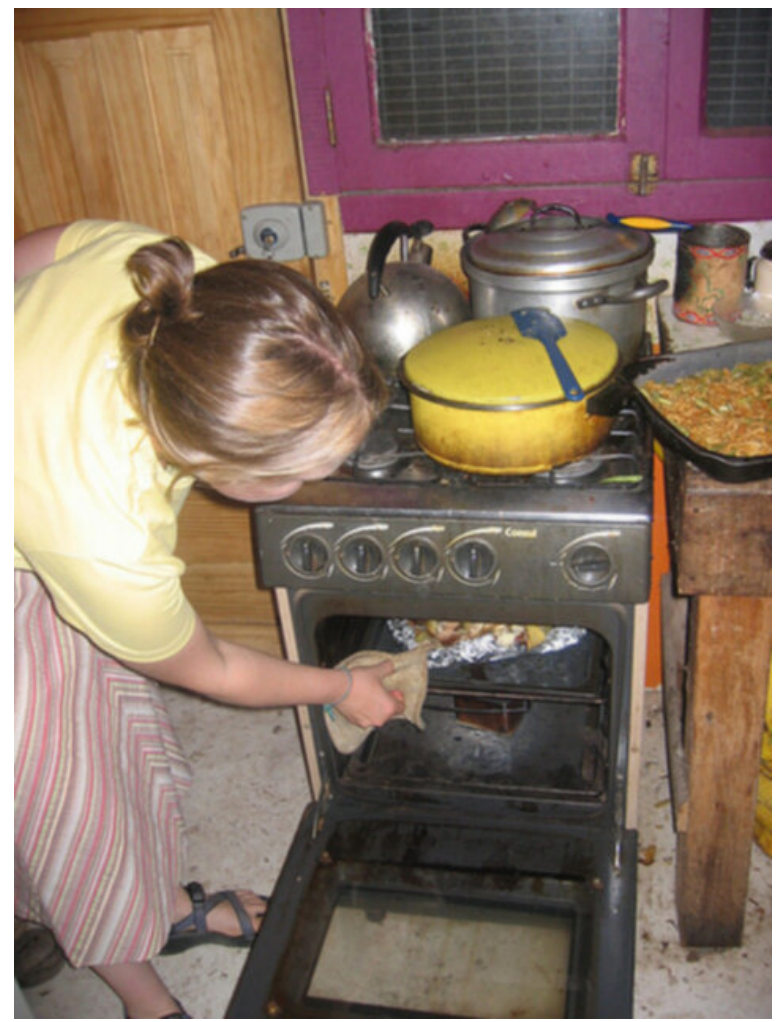


Question:what is made out of wood?

\section{MRR candidate set}

1. wood

2. wood maybe

3. i can not tell

4. cabinets, stove

5. ca n't tell

6. hard to tell

7. i ca n't tell

8. not sure

\section{Top 10 from the remaining NDCG candidates}

1. looks like metal with wood slats

2. looks like a table

3. table

4. the walls

5. ca n't really tell but i would assume so

6. yes

7. yes, it is

8. i think so

9. i ca n't tell but i am guessing a large bin

10. it looks to be scissors and a beer can box

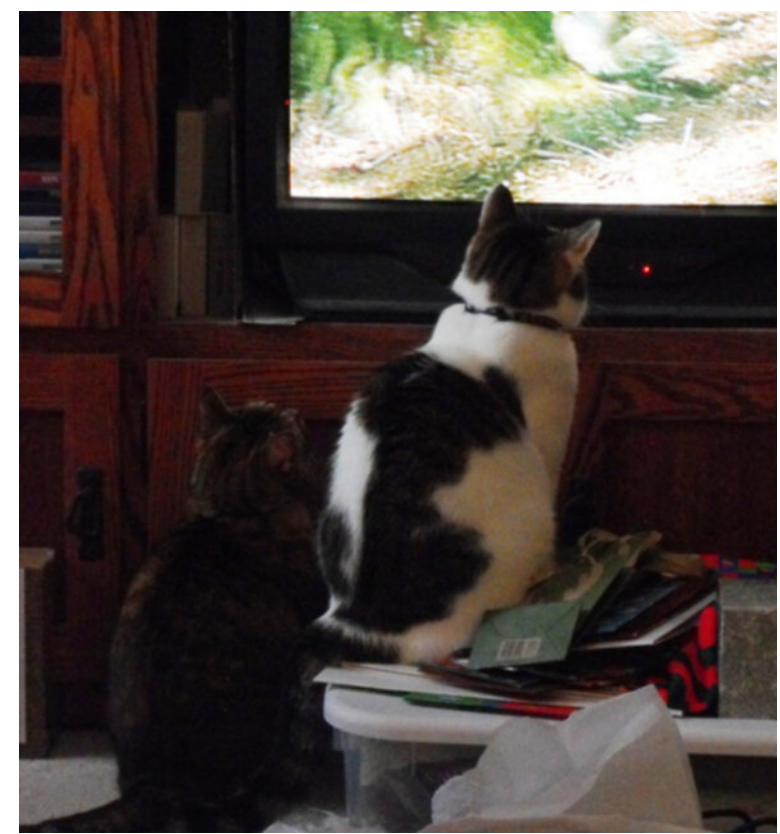

Question:are there laying on the floor?

MRR candidate set
1. no

2. yes

3. nope

4. no there are n't

\section{Top 10 from the remaining NDCG candidates}

1. 1 is

2. no there is not

3. i do n't think so

4. not that i see

5. you can 't see the floor

6. 1 is the other is sitting on top of a container

7. not that i can see

8. ys

9. i think so

10. not really

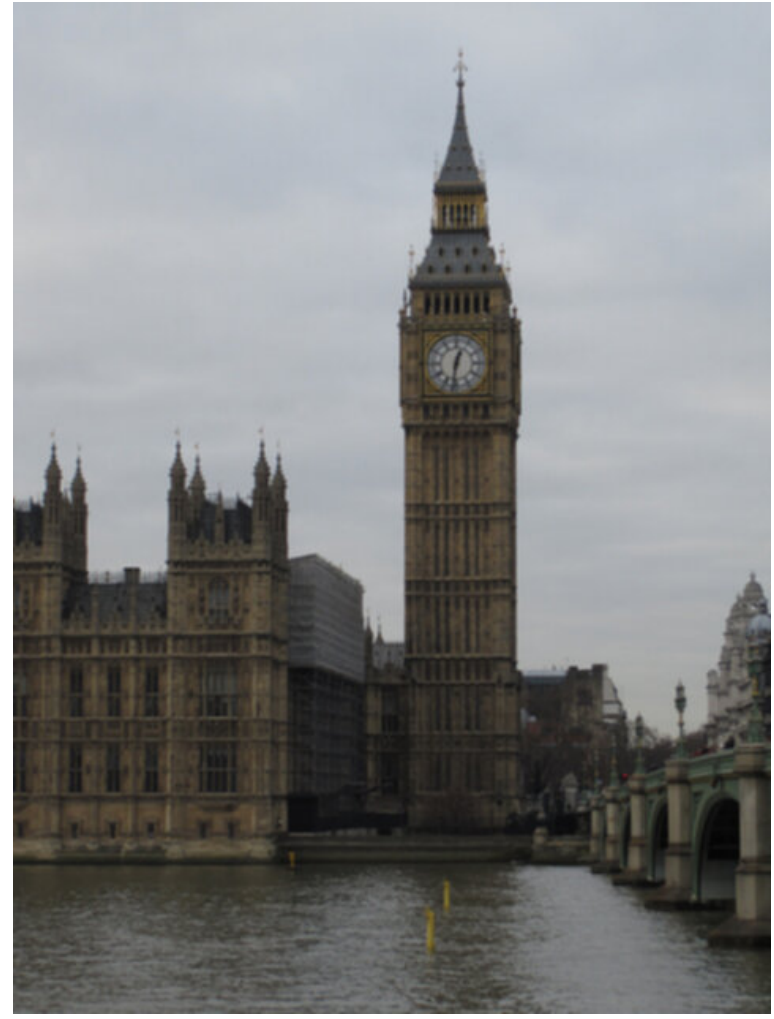

Question:is there a clock?

\section{MRR candidate set}

1. yes

2. no 
3. yes there is

4. yes, there is

5. уер

\section{Top 10 from the remaining NDCG candidates}

1. UNK

2. i think so

3. yes, it is

4. yes it is

5. yes behind

6. yes there are 2

7. yes, several of them

8. yes it 's black

9. yes there is a rug

10. yes on a hamper

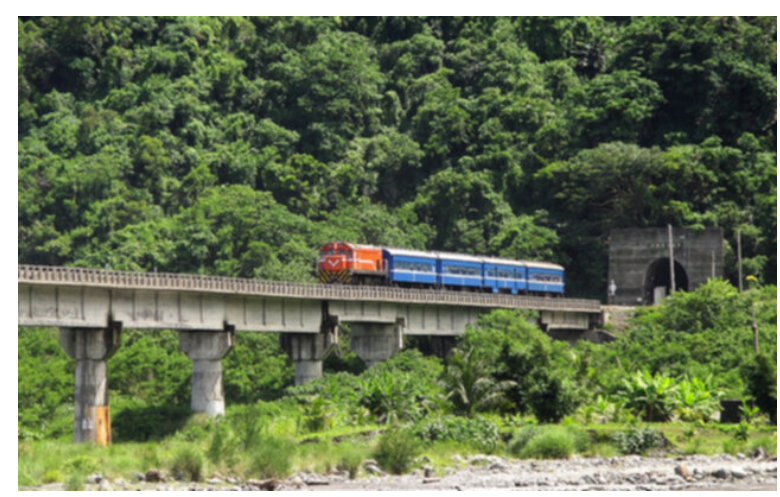

Question:do the trees have leaves?

\section{MRR candidate set}

1. yes

2. yes a lot

3. yes, very

4. yes they do

5. 'yes

\section{Top 10 from the remaining NDCG candidates}

1. yes, they are

2. some have leaves

3. yes a few of them

4. i think so
5. kinda

6. yes, they look fine

7. yes, it is

8. yes, a few

9. green

10. yes it is

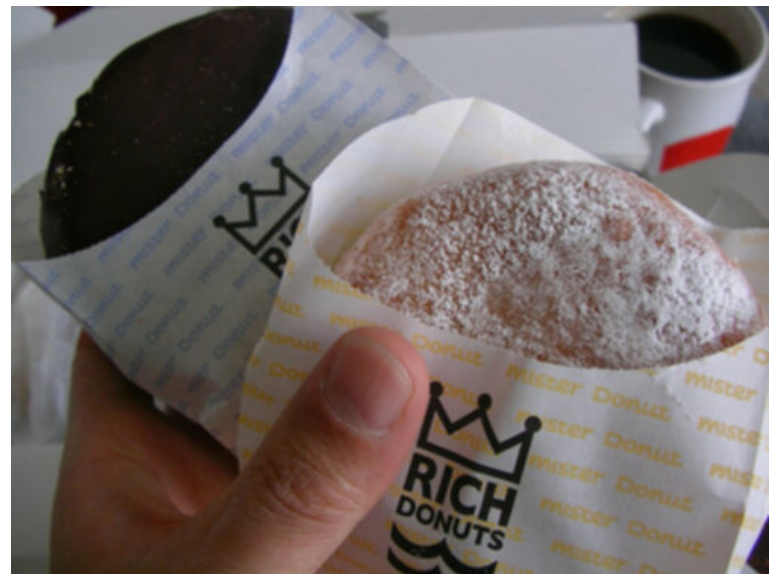

Question:is the chocolate donut frosted? MRR candidate set

1. yes

2. no

3. yes, it is

4. yes it is

\section{Top 10 from the remaining NDCG candidates}

1. it is

2. nope

3. looks like it

4. yes, there 's some white frosting

5. i think it

6. i think so

7. yes, they are in both

8. i do n't think so

9. not completely sure, does n't look like it

10. not that i can tell 


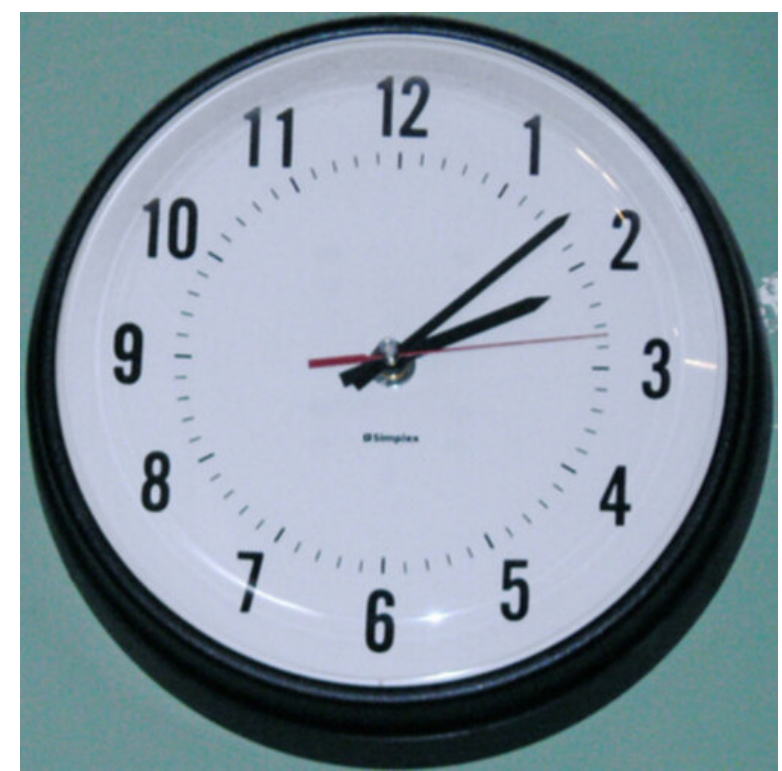

Question:if you were to guess, what building is the image set in ?

\section{MRR candidate set}

1. i can not tell

2. i can not see it from here

3. i ca n't tell

4. ca n't tell

5. not sure

\section{Top 10 from the remaining NDCG candidates}

1. it is in a home

2. maybe an office or a school

3. can not tell since it is outside

4. it looks like a restaurant

5. this was not taken by a professional

6. in a parking lot somewhere

7. i can not tell by the picture how nice the restaurant is

8. it is hard to tell, i can only see the table cloth

9. no, you can not

10. he is n't in a kitchen

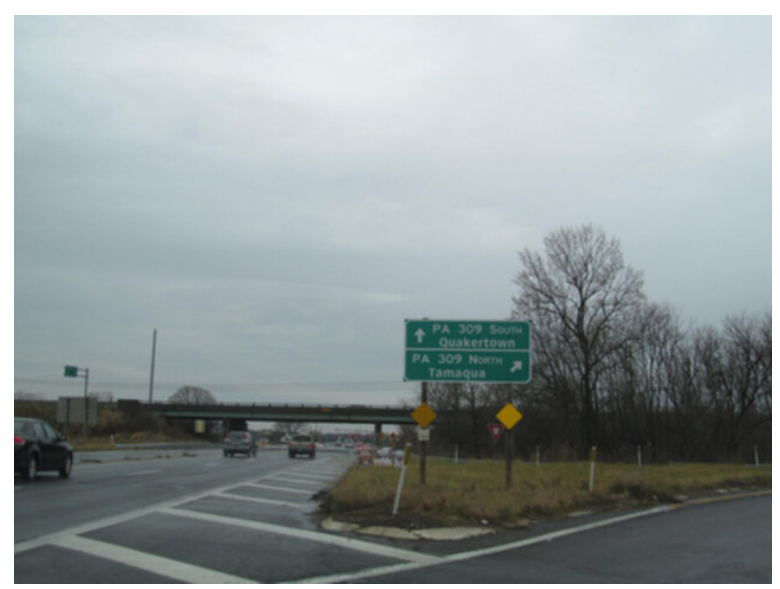

Question:what is on the sign ? MRR candidate set

\section{1. letters}

\section{1 says stop and the other says danger}

3. pictures

4. i do n't understand the question

5. a flickr UNK

Top 10 from the remaining NDCG candidates

1. look like a game

2. land in the background

3. some website not sure

4. UNK

5. not sure

6. a track and a little gate building

7. a few penguins out in penguin element

8. yes, i see like 6 billboard signs

9. it 's a dessert

10. large rocks on the side of the road 


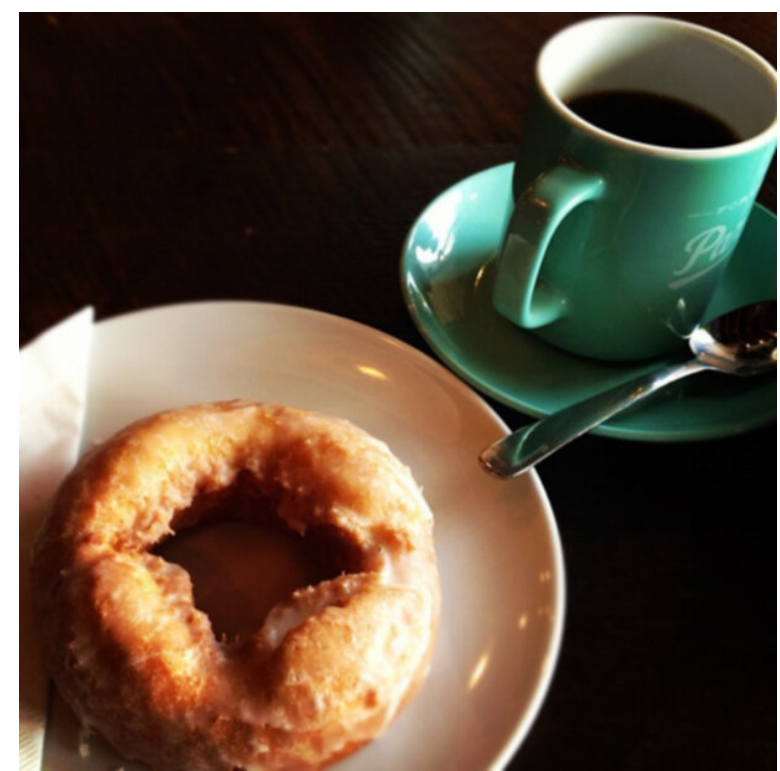

Question:is there any cream or sugar near the mug ?

\section{MRR candidate set}

1. no, there is a spoon next to the mug on the plate however

2. yes, a small scoop of yellowish ice cream on top of the pastry

3. no there is not

4. no that i can see

5. not that i can see

\section{Top 10 from the remaining NDCG candidates}

1. no

2. nope

3. not visible in the picture, no

4. i do n't think so

5. yes

6. yes there is

7. i can not tell

8. 0

9. mug

10. not really

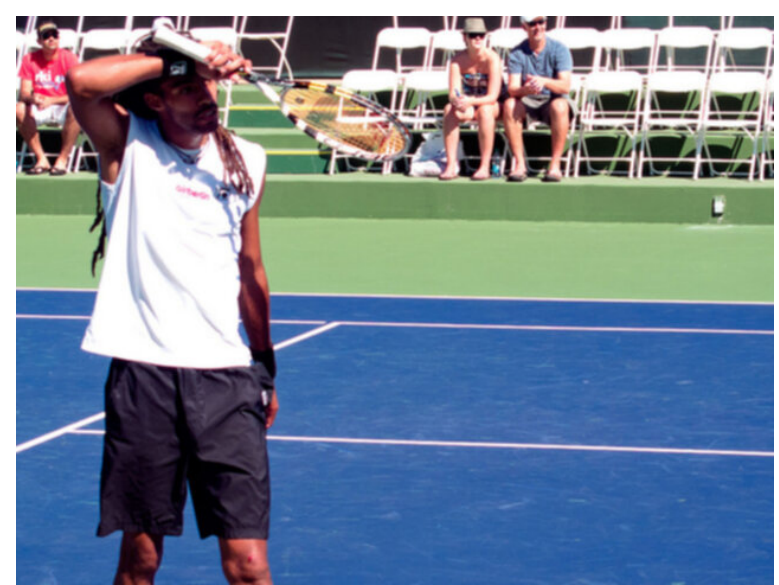

Question:can you see his opponent?

MRR candidate set

1. no

2. no i can not

3. no, i can not

4. nope

Top 10 from the remaining NDCG candidates

1. no, not at all

2. not in my view

3. no just 1 person

4. i do n't think so

5. not that i can see

6. i'm not sure

7. no, only people

8. not really

9. there is no scoreboard

10. sure why not

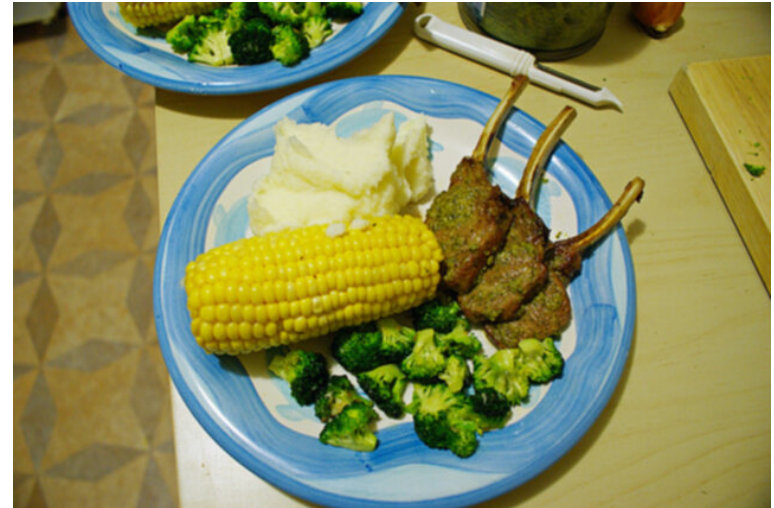

Question:are any beverages nearby? MRR candidate set 
1. no

2. not that i can see

3. nope

4. not that i can tell

5. 0 that are visible

Top 10 from the remaining NDCG candidates

1. i do n't think so

2. 0

3. no, thankfully

4. yes

5. i do n't know

6. i think so

7. it is a close up of the plate

8. not really

9. i can not tell

10. yes 1

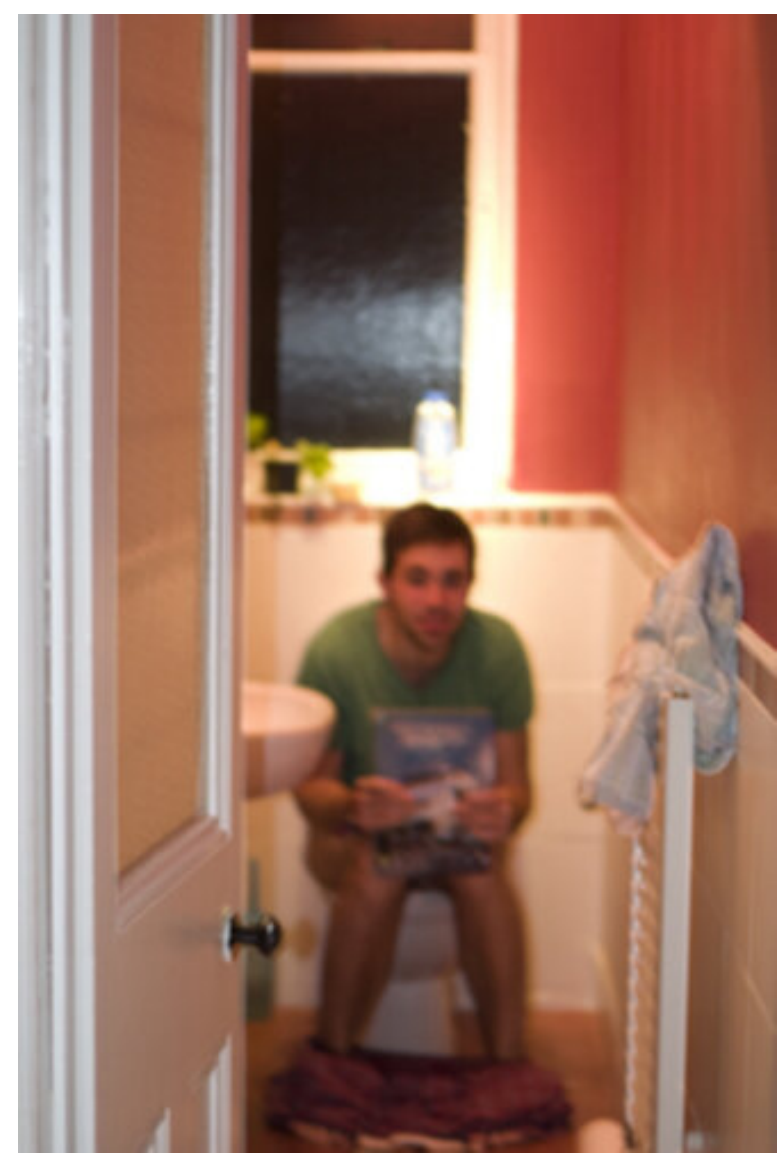

Question:is this in color?

MRR candidate set
1. yes

2. yes, it is

3. yes it is

4. yes in color

5. yes it appears to be

Top 10 from the remaining NDCG candidates

1. it is

2. yes, i think so

3. yea

4. i think so

5. yes* and yes

6. appears to be

7. $\mathrm{i}$ believe so

8. most

9. hell yeah

10. most likely

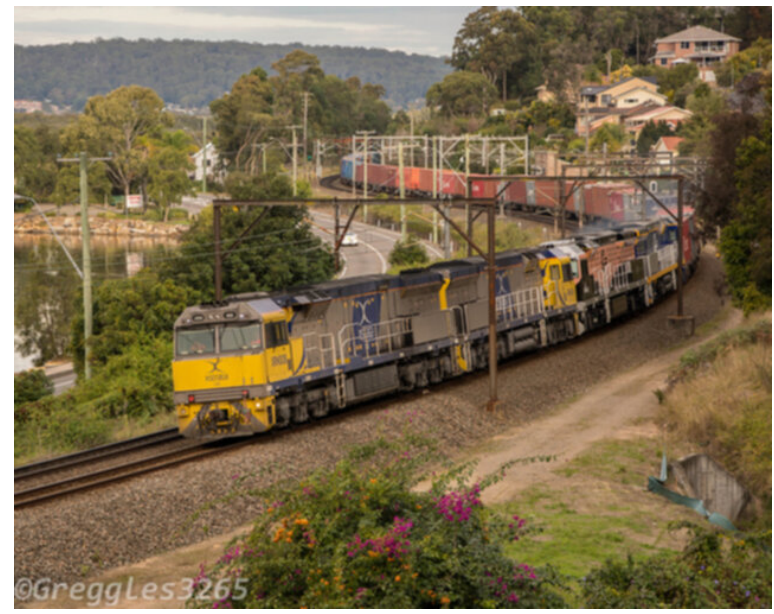

Question:what is in background ?

MRR candidate set

1. buildings houses like a village

2. trees and mountains

3. houses

4. lots of trees and bushes

5. trees

Top 10 from the remaining NDCG candidates 
1. a house

2. a UNK and the sky

3. tall grass

4. yes, there are a few buildings

5. rocks and small bushes

6. the brick building

7. a tree

8. yes

9. not sure

10. out in the open

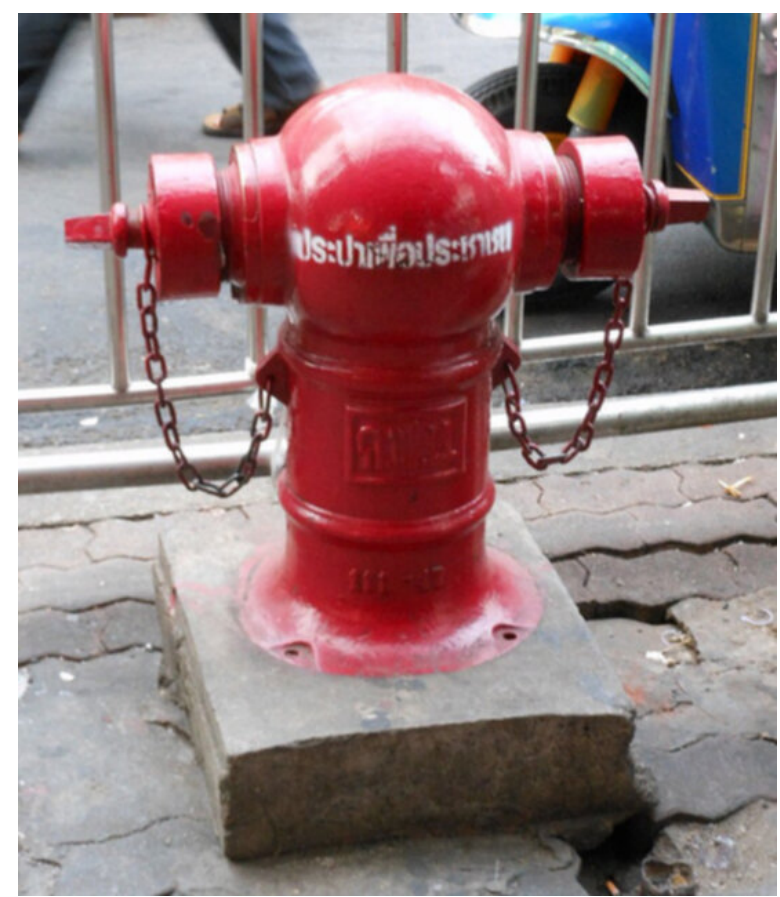

Question:is there anyone on the motorcycle? MRR candidate set

1. no

2. i can not tell

3. i can not see

4. not that i can see

5. nope

\section{Top 10 from the remaining NDCG candidates}

1. i ca n't tell

2. ca n't tell

3. i do n't think so
4. no 1

5. i ca n't see any but probably

6. not sure

7. it 's too blurry to tell

8. 0

9. only his feet are visible

10. no people

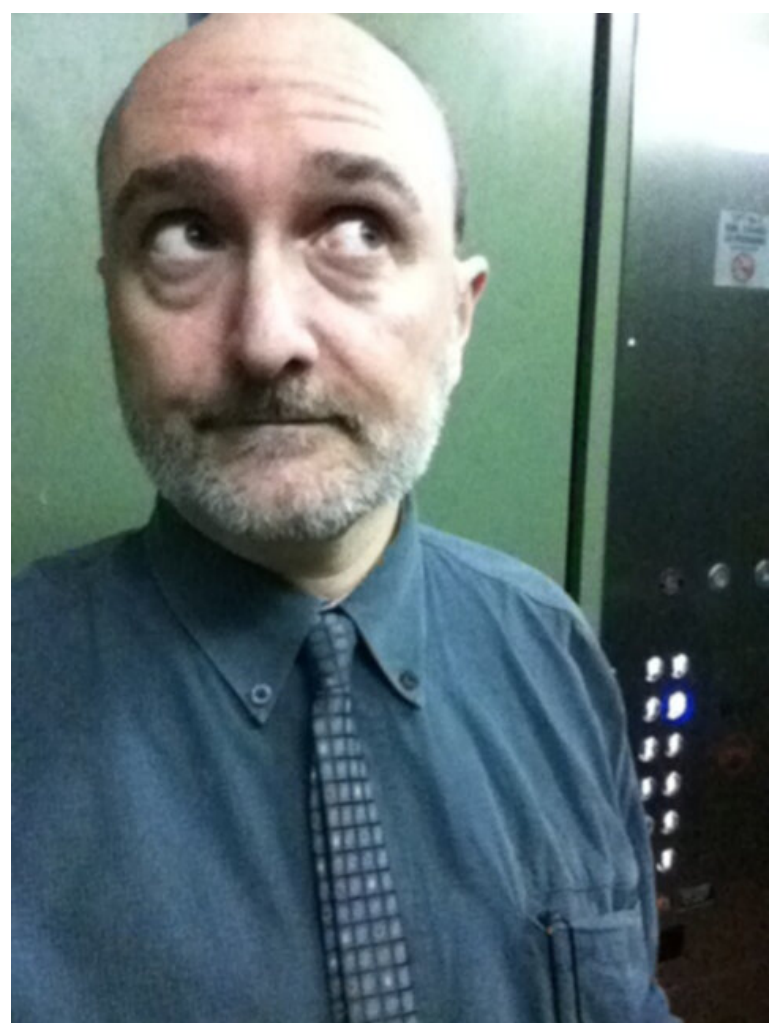

Question:is the elevator door open? MRR candidate set

1. no

2. yes

3. i can not tell

4. i ca n't tell

5. ca n't tell

6. ca n't see

7. not sure

Top 10 from the remaining NDCG candidates

1. ca n't really tell

2. no it is n't 
3. no, it is not

4. yes it is

5. not in the picture no

6. yes, it is

7. no not open

8. no it's not

9. i think so

10. it looks like it

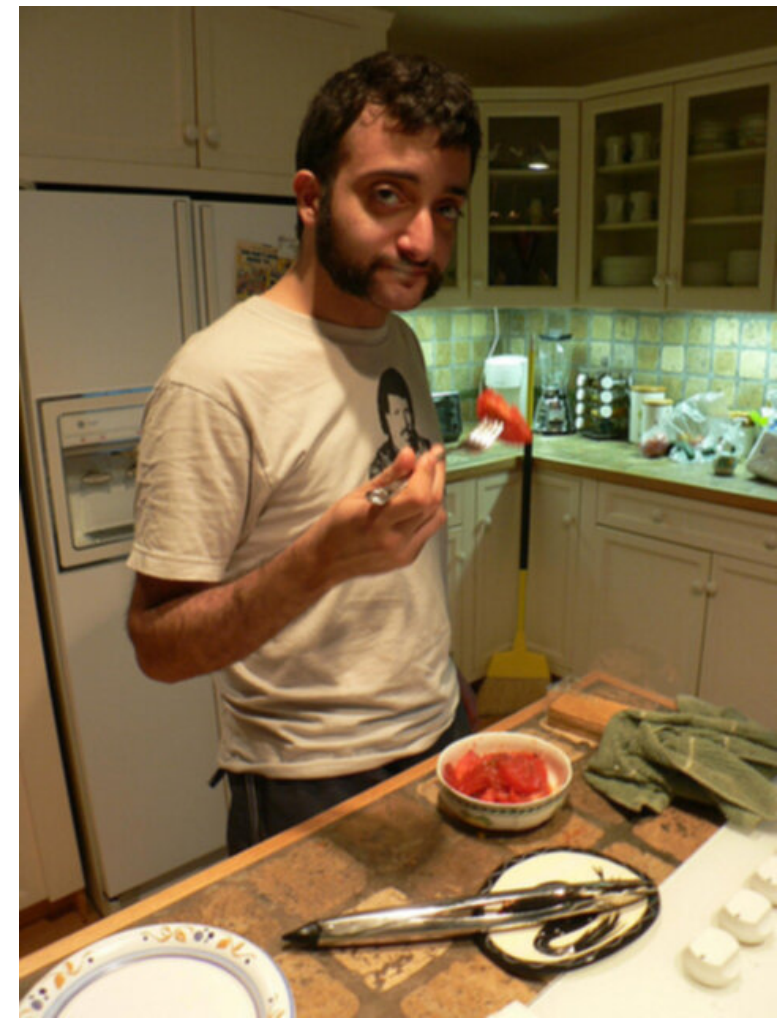

Question:is he eating over the sink?

\section{MRR candidate set}

1. no he is eating over the island counter

2. no he is n't

3. no he is not

4. no he isn' $t$

5. no, he is not

6. nope

\section{Top 10 from the remaining NDCG candidates}

1. no

2. no not eating
3. i do n't think so

4. yes

5. yes he is

6. i can not tell

7. i ca n't tell

8. yes, he is

9. not that i can see

10. i ca n't see that part

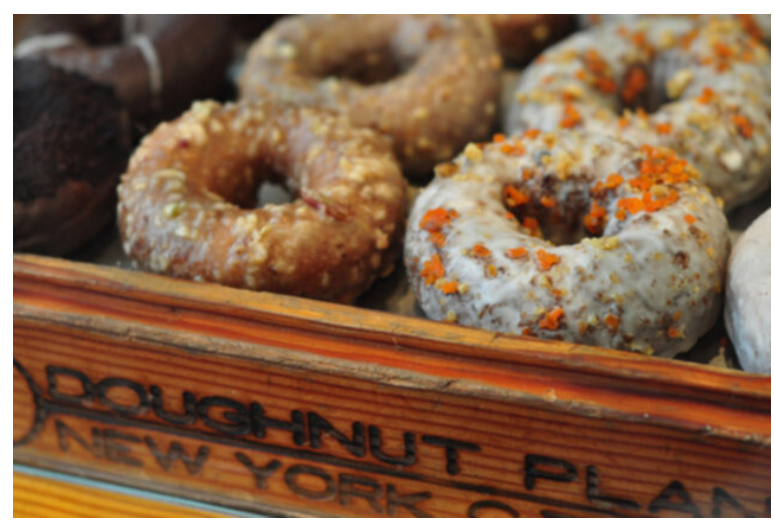

Question:can you tell if any donuts have been taken from the box?

MRR candidate set

1. no

2. i can not tell

3. i do n't think so

4. no i can not

5. nope

6. not that i can see

\section{Top 10 from the remaining NDCG candidates}

1. 0

2. no i can not tell

3. no, ca n't tell

4. i ca n't tell

5. not really

6. there are a few but don' $t$ know the exact number

7. yes 
8. not sure

9. ca n't tell

10. yes 1

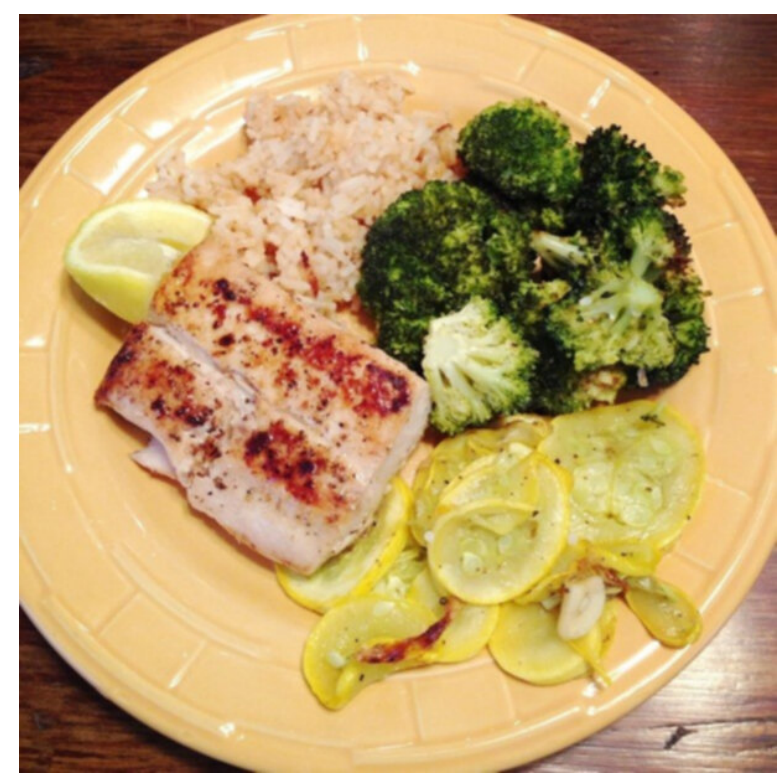

Question:do you see any hand of a person ?

MRR candidate set

1. no

2. no there are no people visible

3. nope

4. no you can not see any

Top 10 from the remaining NDCG candidates

1. no people are visible

2. no i don' $\mathrm{t}$

3. there are no people

4. no people at all

5. no people

6. not that $\mathrm{i}$ can see

7. no , just the food

8. 0 at all

9. 0

10. there are 0 of those

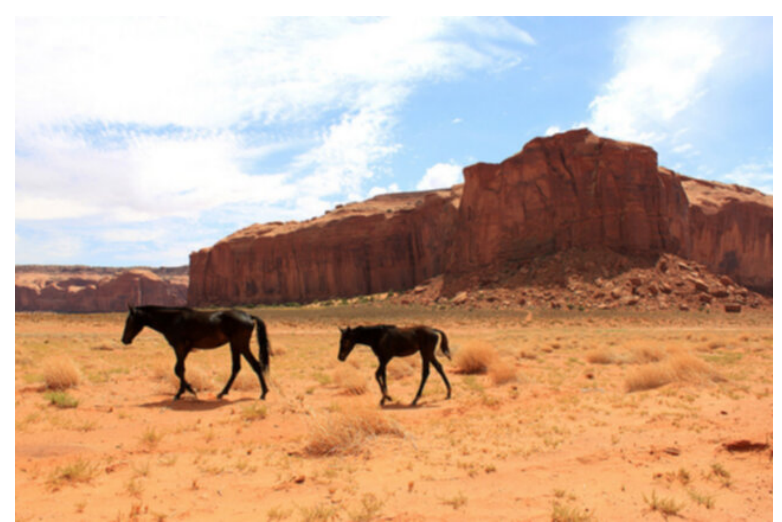

Question:are there any cacti ?

MRR candidate set

1. no

2. 0

3. nope

4. not that $\mathrm{i}$ can see

5. no there are not

Top 10 from the remaining NDCG candidates

1. 0 at all

2. no i do n't see any

3. i don' t see any

4. not any in the picture

5. i do n't think so

6. sadly, no

7. non

8. do n't have a clue

9. yes

10. no, just the grasses

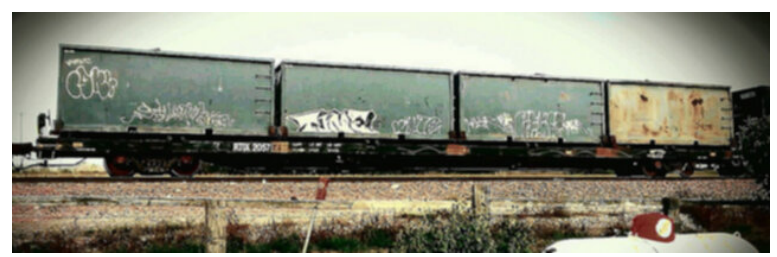

Question:is there writing on the train ? MRR candidate set

1. there is some spray painted graffiti

2. yes

3. yes, in english and foreign 
4. yes, but ca n't make it out

5. yes there is

Top 10 from the remaining NDCG candidates

1. just numbers

2. there may be but it is too difficult to see

3. amtrak

4. yes, it is

5. not that $\mathrm{i}$ can see

6. i think so

7. no

8. nope

9. no there is not

10. yes, a little

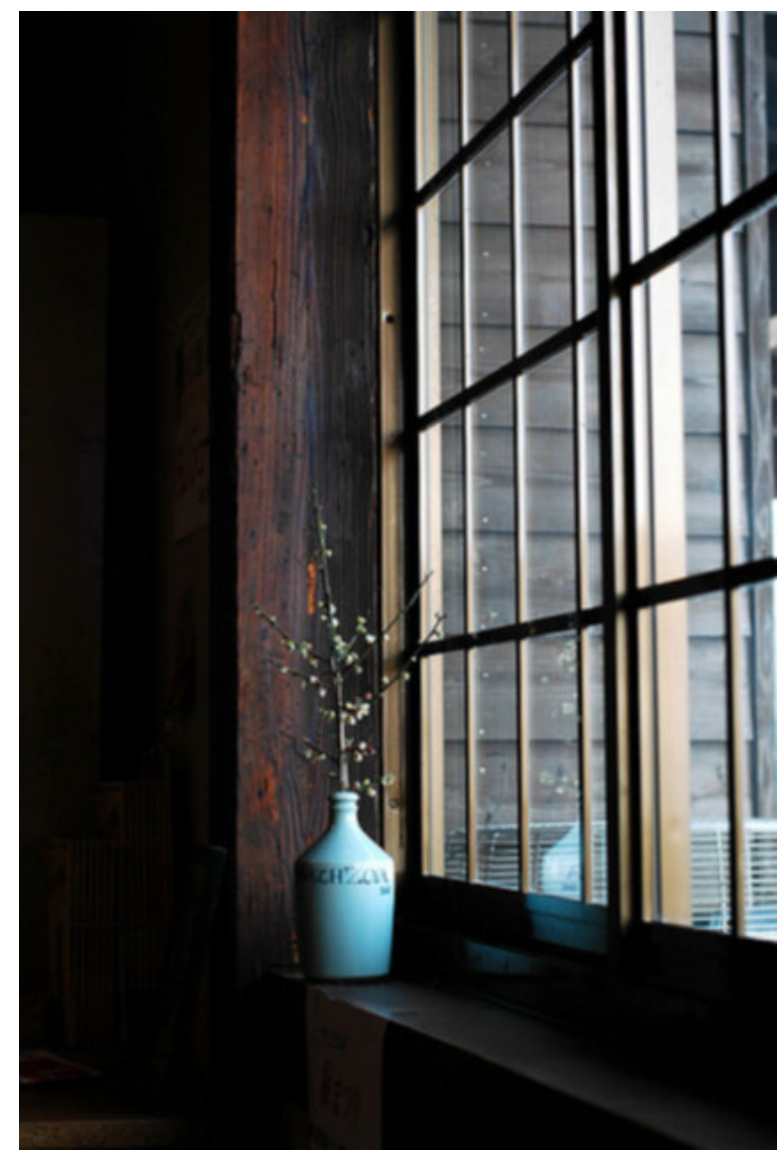

Question:does it look sunny?

MRR candidate set

1. yes

2. yes, it is
3. yes it does

4. no

5. no, it is n't

6. not really

\section{Top 10 from the remaining NDCG candidates}

1. i can not tell

2. it looks mostly sunny

3. nope

4. i ca n't tell

5. i guess so, it is hard to tell

6. yes, i 'd say so

7. not that i can see

8. not sure

9. ca n't tell

10. i do n't think so

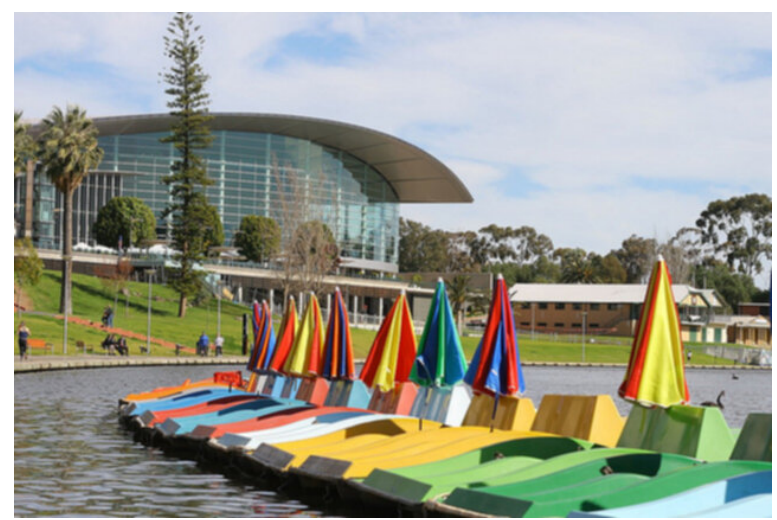

Question:is the lake quite full? MRR candidate set

1. yes very full

2. yes

3. it 's too far away to tell

4. i can not tell

5. i ca n't tell

6. it seems so

Top 10 from the remaining NDCG candidates

1. i would say yes

2. ca n't tell 
3. yes it is

4. looks like it

5. yes, it is

6. i think so

7. i only see a portion of it, so ca n't tell

8. yes i can see it

9. not sure

10. yes absolutely

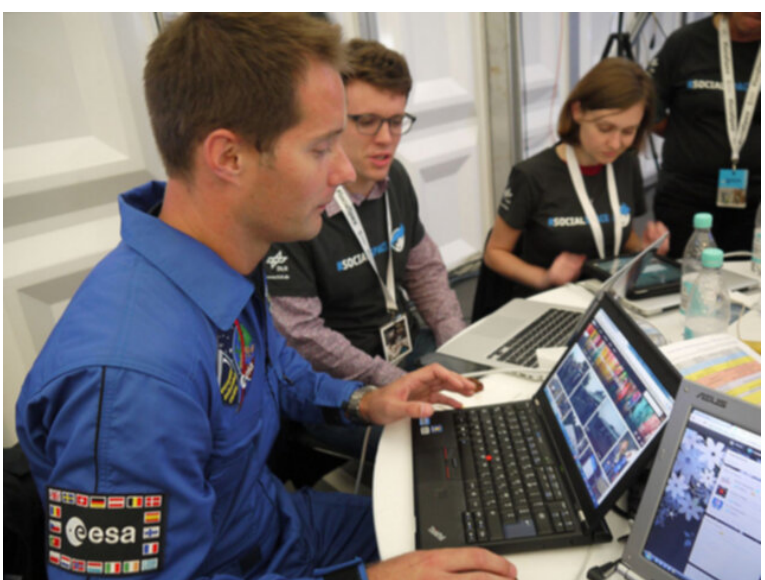

Question:can you see the floor?

MRR candidate set

1. no

2. no, you can not

3. no, i ca n't

4. nope

5. no i ca n't

\section{Top 10 from the remaining NDCG candidates}

1. no you can not

2. no it is not visible

3. not really

4. no, i ca n't see the ground

5. yes the floor is visible

6. yes, the floors are visible

7. i do n't think so

8. no this is inside on a table

9. yes
10. a little bit

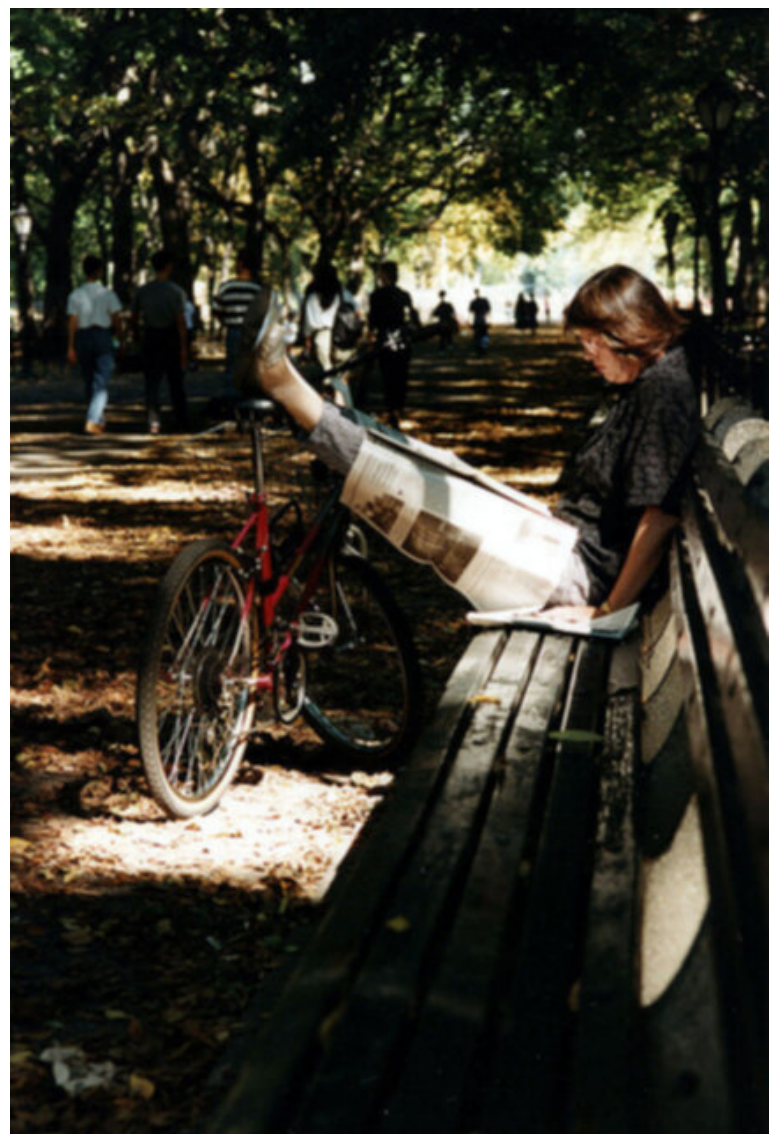

Question:are there any other people in the picture ?

\section{MRR candidate set}

1. yes there are several people walking in the background

2. yes

3. there are people in the background

4. there are a few

5. yes lots of them

\section{Top 10 from the remaining NDCG candidates}

1. there are people

2. ye

3. there are trees in the background, yes

4. yes, a couple ball boys

5. yes, audience

6. yes, it is

7. i think so

8. no other people can be seen in the photo 
9. there is 1 man in the background

10. there are buildings far off in the distance, it is the only close 1

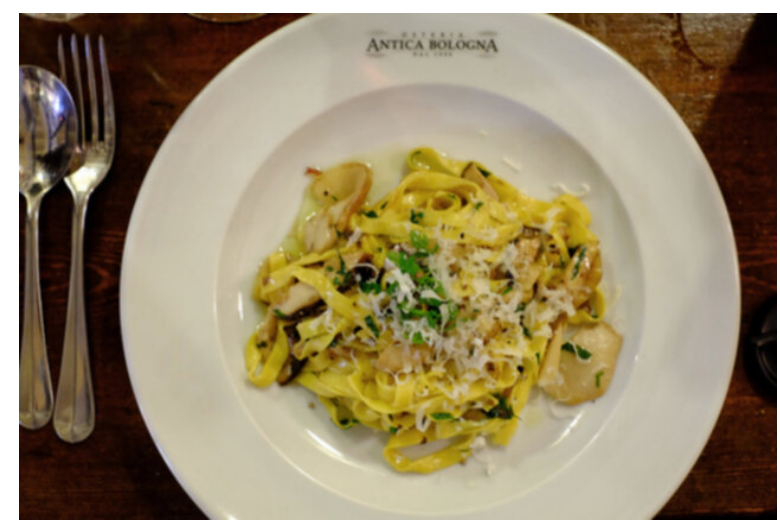

Question:is the noodles beside the chicken? MRR candidate set

1. no it is all mixed together

2. i can not tell

3. yes

4. no

5. yes it is

6. yes, it is

7. yes!

\section{Top 10 from the remaining NDCG candidates}

1. no it is n't

2. across from it

3. nope

4. i think so

5. possibly yes

6. i do n't believe so

7. not that i can see

8. i do n't think so

9. i think it is , but hard to say for sure

10. not really

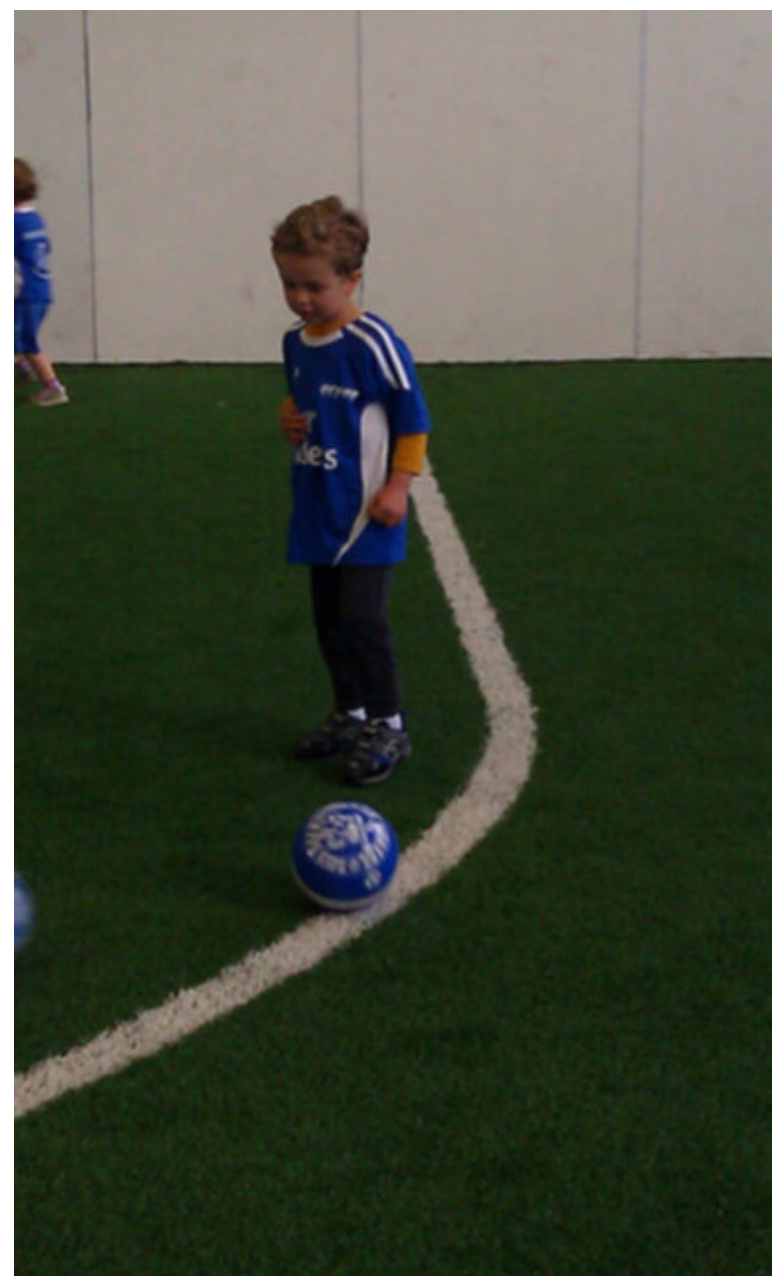

Question:can you read the writing on the ball ? MRR candidate set

1. no, i ca n't make it out

2. no

3. i ca n't, it 's blurry

4. it's in another language

5. yes i can but it is in a foreign language

\section{Top 10 from the remaining NDCG candidates}

1. yes

2. yes i can

3. nope

4. there are a lot of small words it is hard to read

5. yes i do

6. not really

7. i can not tell

8. there are several, but i ca n't see the names 
9. ca n't see

10. i ca n't tell

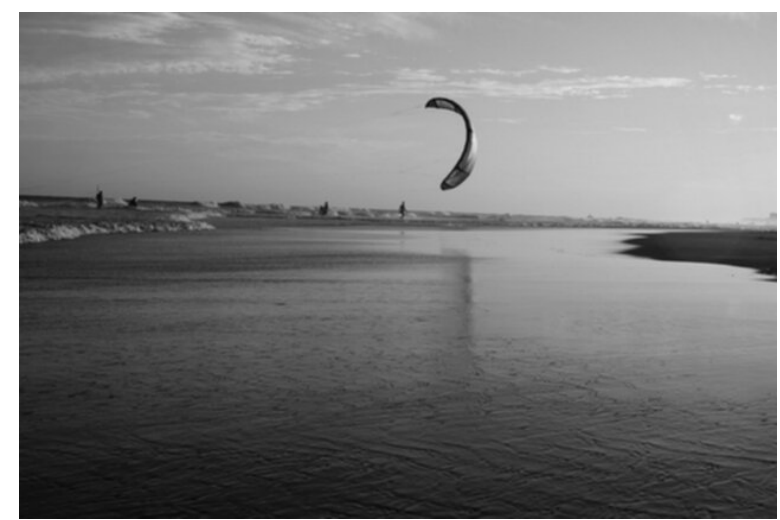

Question:is the water clear or dirty?

MRR candidate set

1. i can not tell

2. it appears clean

3. i ca n't tell

4. very clear

5. can not tell

6. ca n't tell

Top 10 from the remaining NDCG candidates

1. it is clean

2. clean

3. neither

4. can barely see the water

5. not sure

6. it 's a fairly deep bluish-green, does n't look like you could see through it

7. it does not look brown

8. grey

9. choppy

10. looks pretty gross

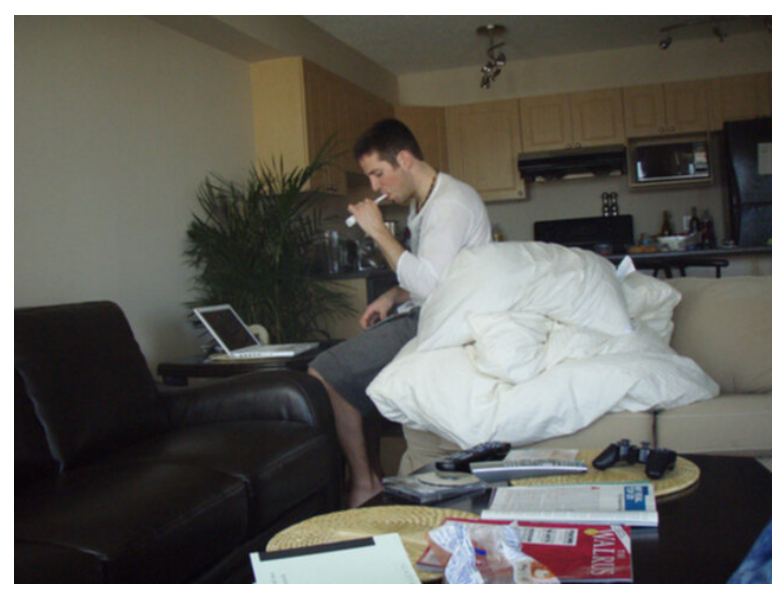

Question:is it a living room?

MRR candidate set

1. yes

2. i think so

3. it appears to be

4. yes it is

5. yes i think so

\section{Top 10 from the remaining NDCG candidates}

1. looks like it

2. yes, it is

3. seems to be

4. it looks like it could be

5. it could be

6. maybe

7. it is

8. might be, not sure

9. no

10. not sure

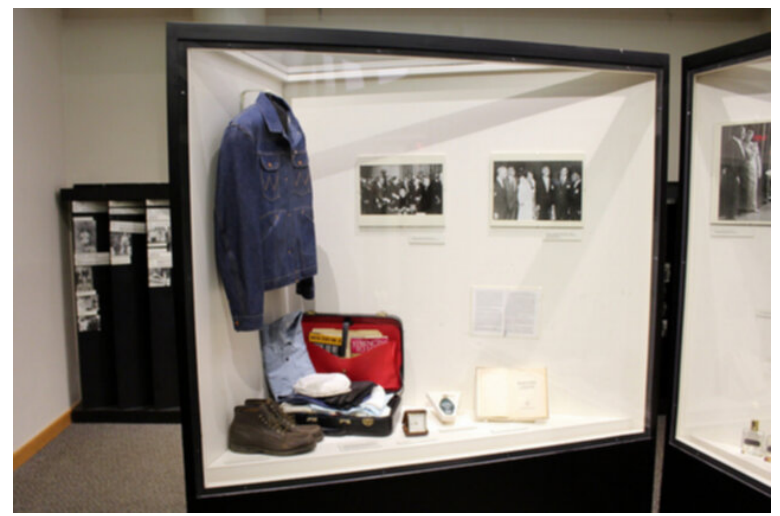

Question:what color are the boots?

MRR candidate set 
1. brown

2. black

3. i ca n't see from the angle

4. i can not tell

5. i ca n't tell

\section{Top 10 from the remaining NDCG candidates}

1. gold

2. beige and brown

3. brown and green

4. ca n't tell

5. not sure

6. the look like they are black and white

7. beige

8. looks like they are red

9. gray

10. light grey

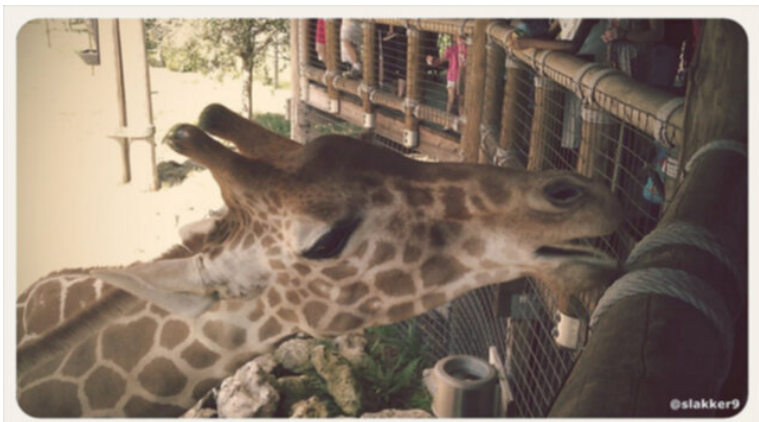

Question:can you see the clouds?

\section{MRR candidate set}

1. no

2. no i can not see the sky

3. no ca n't see sky

4. no, i ca n't see the sky

Top 10 from the remaining NDCG candidates

1. no i ca n't

2. no i can not

3. nope

4. i ca n't
5. i can not

6. i can not tell

7. no the sky is clear

8. i ca n't tell

9. i do n't think so

10. not that $i$ can see

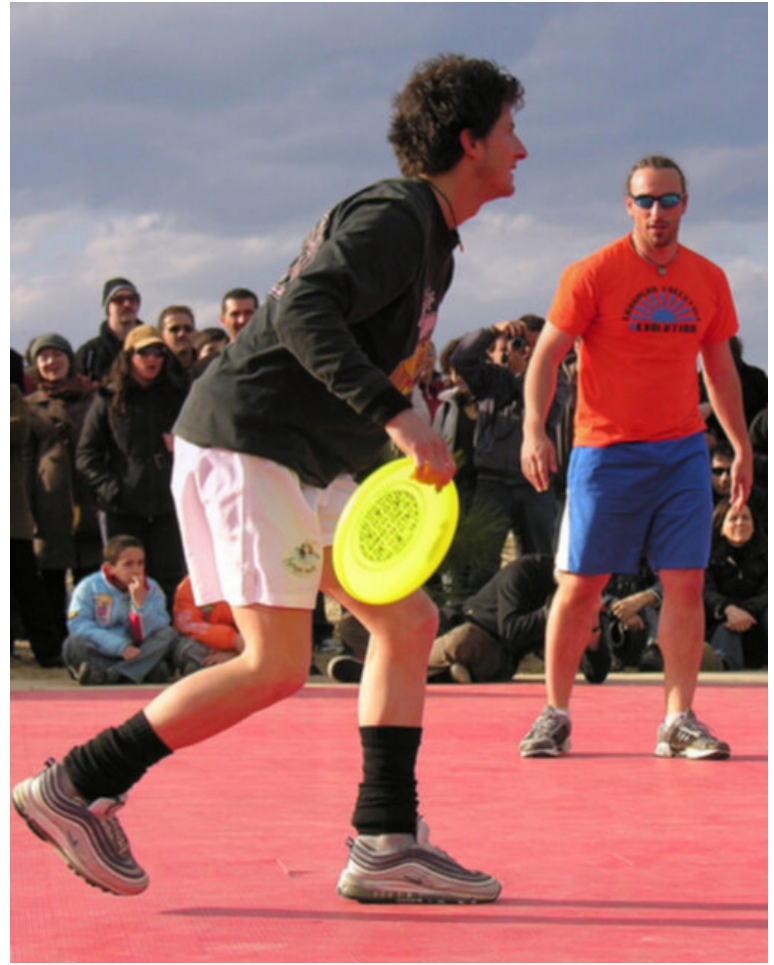

Question:what color is the frisbee ?

MRR candidate set

1. yellow

2. yellow and black

3. green

4. yellow and red

5. green yellow and red

\section{Top 10 from the remaining NDCG candidates}

1. yellow as well as his shirt

2. yes, it is

3. olive green

4. yes

5. yes it is

6. red, yellow, white and black 
7. a round beige 1

8. just about every color UNK

9. it is white

10. white

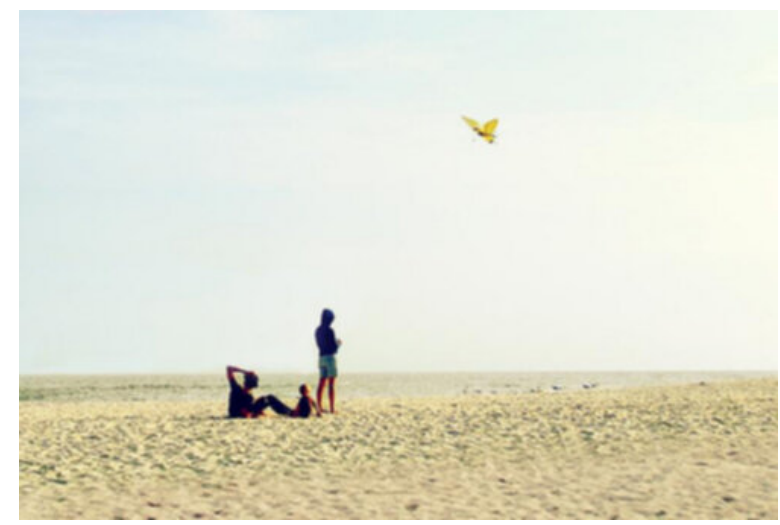

Question:how old is the kid?

\section{MRR candidate set}

1. not sure maybe $3-4$

2. around eleven

3. can not tell

4. ca n't tell

5. i can not say

6. i can not tell

\section{Top 10 from the remaining NDCG candidates}

1. ca n't tell from the picture

2. i ca n't tell

3. probably around 2

4. not sure

5. about $2-3$

6. maybe 1

7. he looks young maybe 6 or 7

8. i ca n't make out their age

9. maybe 8

10. the boy looks about 7 or 8

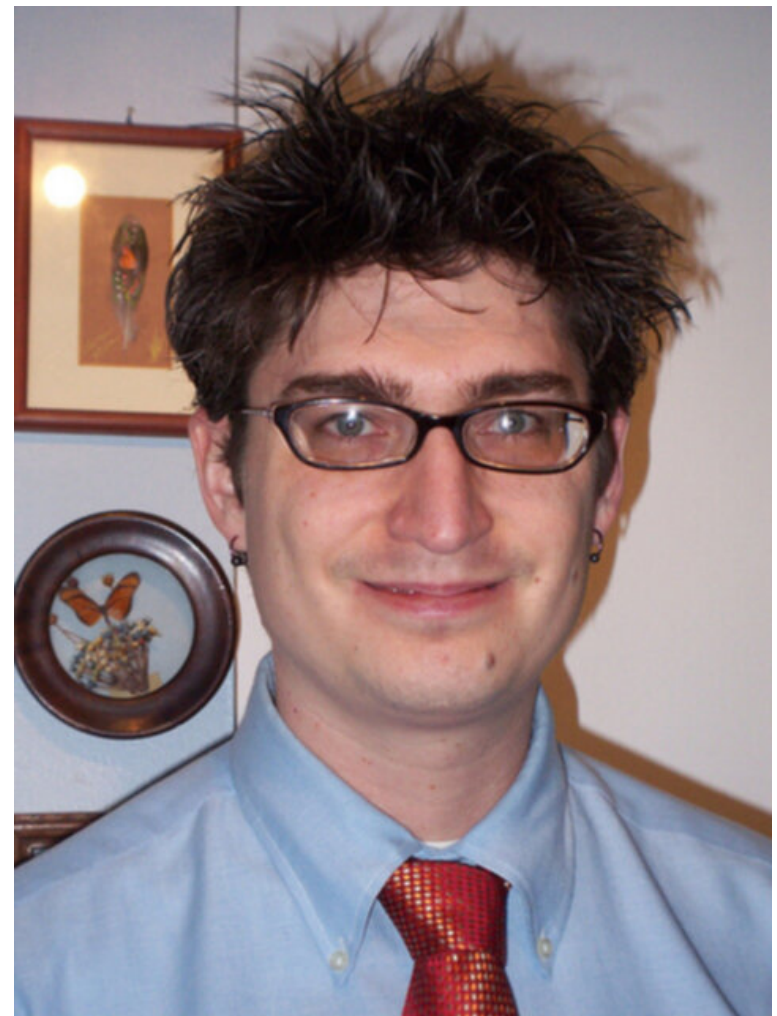

Question:is he indoors?

MRR candidate set

1. yes

2. i think so

3. yes, he is

4. yep

\section{Top 10 from the remaining NDCG candidates}

1. yes, $\mathrm{i}$ think so

2. he 's inside

3. seems to be

4. it is indoors

5. it looks like it is

6. yes it looks like it was taken indoors

7. yes it is

8. yes, it is

9. looks like

10. inside i think 


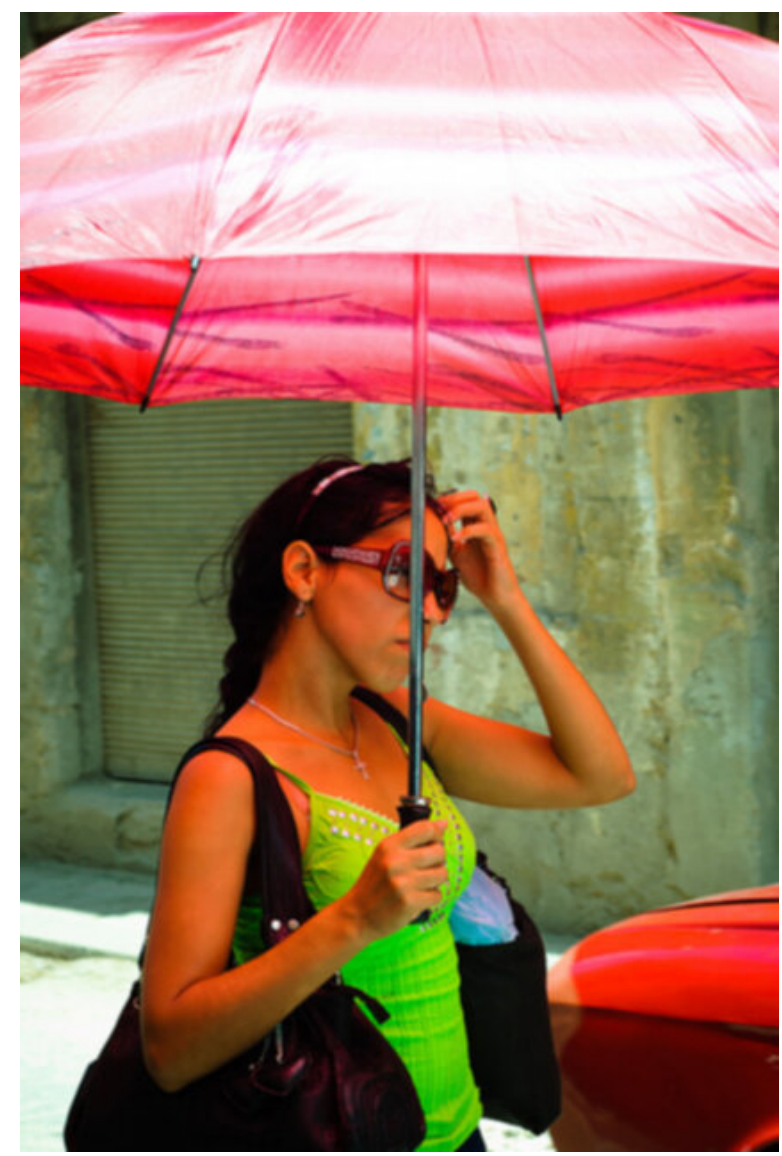

Question:is the woman old?

MRR candidate set

1. no

2. it is hard to tell, but i would say she is in her twenties

3. probably in her 30 s

4. not very young

5. i do n’t know

6. nope

\section{Top 10 from the remaining NDCG candidates}

1. i do n't think so

2. no, she seems like she could be in early college

3. not sure

4. doubtful

5. middle aged i 'd say

6. no not really

7. ca n't tell
8. not really

9. not that $i$ can see

10. i think so , ca n't see her face well

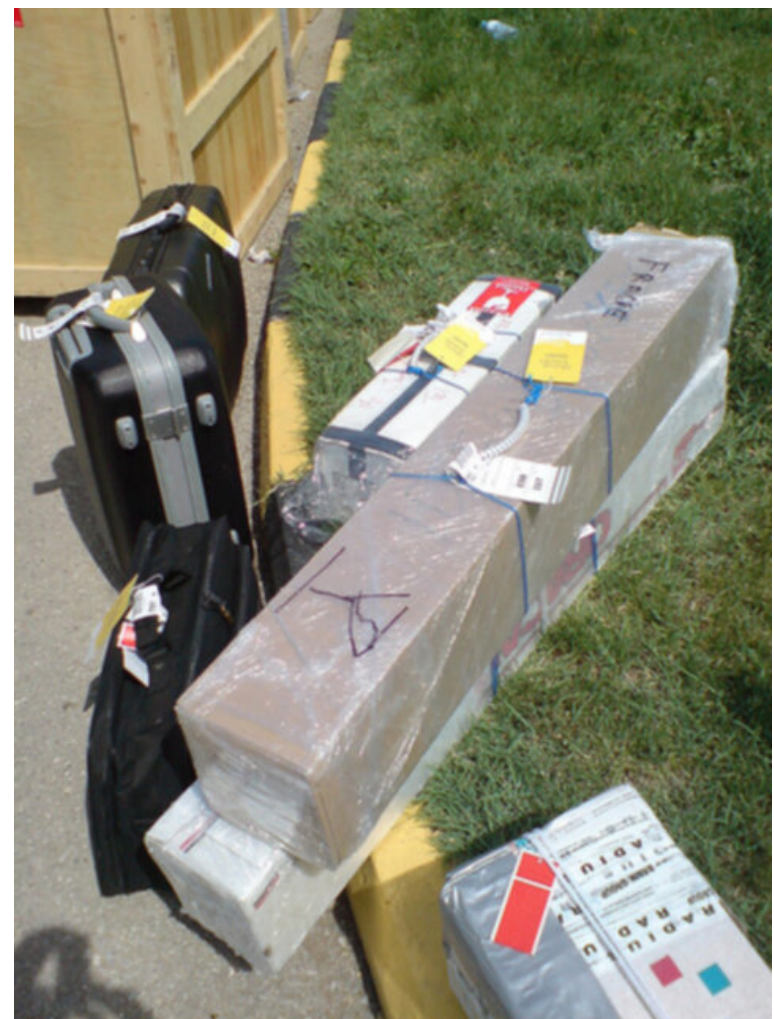

Question:are there buildings ?

MRR candidate set

1. no

2. not that i can see

3. i ca n't see any

4. nope

\section{Top 10 from the remaining NDCG candidates}

1. no there are n't

2. it does n't seem like there are any

3. 0 seen

4. 0

5. ca n't see

6. i do n't think so

7. no, on a sidewalk of a street

8. no it is a close up of them

9. not really 
10. yes

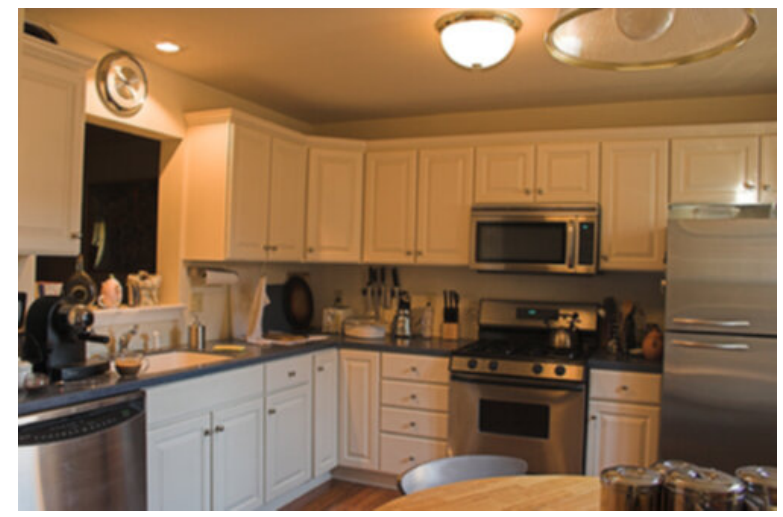

Question:what color are the countertops ?

MRR candidate set

1. white

2. beige

3. they look beige

4. they are white

5. stainless steel

\section{Top 10 from the remaining NDCG candidates}

1. i think off white

2. black

3. like a beige

4. dark wood

5. brown

6. $\tan$

7. eggshell

8. egg white

9. variety of colors

10. black i think

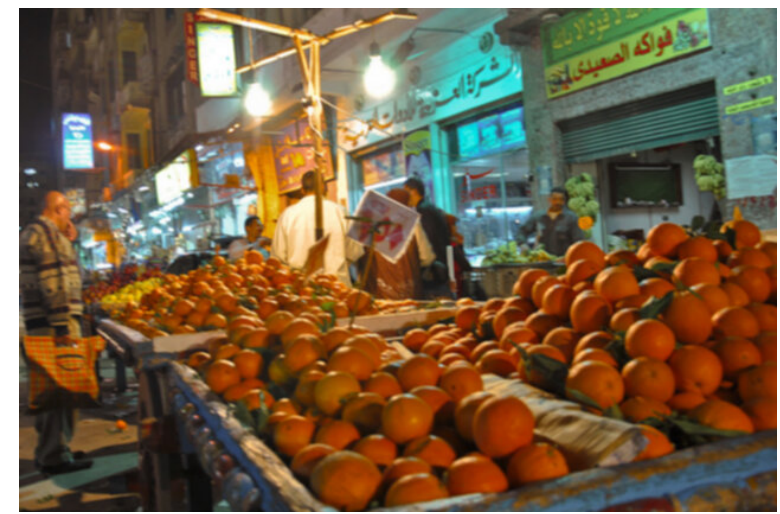

Question:do you see a scale?

MRR candidate set
1. no

2. yes

3. nope

Top 10 from the remaining NDCG candidates

1. no i do n’t

2. i do n’t

3. no i do not

4. no i don' $t$

5. no,

6. i do n't think so

7. not in the picture

8. not that i can see

9. no it doesn' $t$ look like it

10. not really

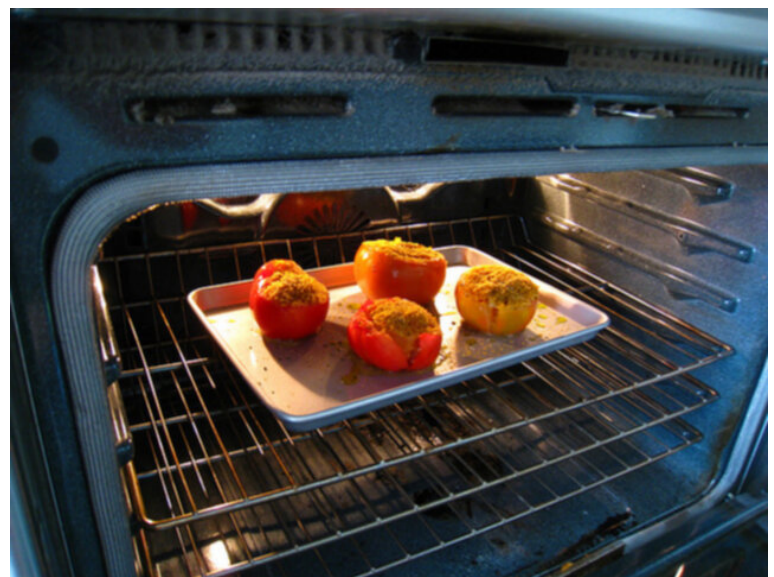

Question:can you tell what they are stuffed with? MRR candidate set

1. no, they are in an self serving orange bottle, it almost looks like a fruit drink

2. i can not tell

3. no

4. not really

5. nope

Top 10 from the remaining NDCG candidates

1. i ca n't tell

2. not sure 
3. not that i can see

4. only the top, which looks to be crumbs for some reason beyond me

5. ca n't tell

6. i do n't see any

7. no the big ones

8. yes

9. i do n't think so

10. it looks like a seasoned french bread

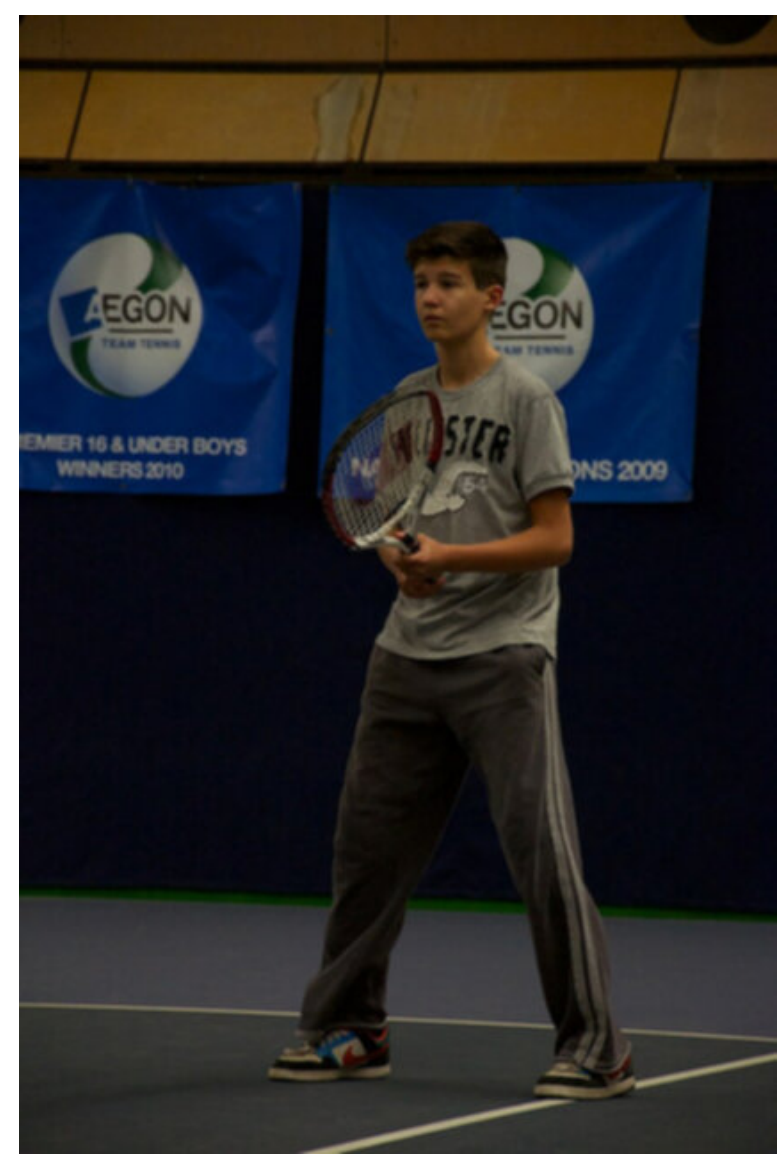

Question:what color are the sweatpants?

\section{MRR candidate set}

1. grey and white

2. grey

3. gray

4. brown

5. black

6. they are khaki

Top 10 from the remaining NDCG candidates
1. gray mostly

2. not clear

3. 1 is black and the other is grey

4. 1 is tan and the other is brown

5. they 're gray metal

6. look orange or tan

7. grey, black, white

8. 1 is dark, the other a lighter brown

9. black and gold

10. not sure

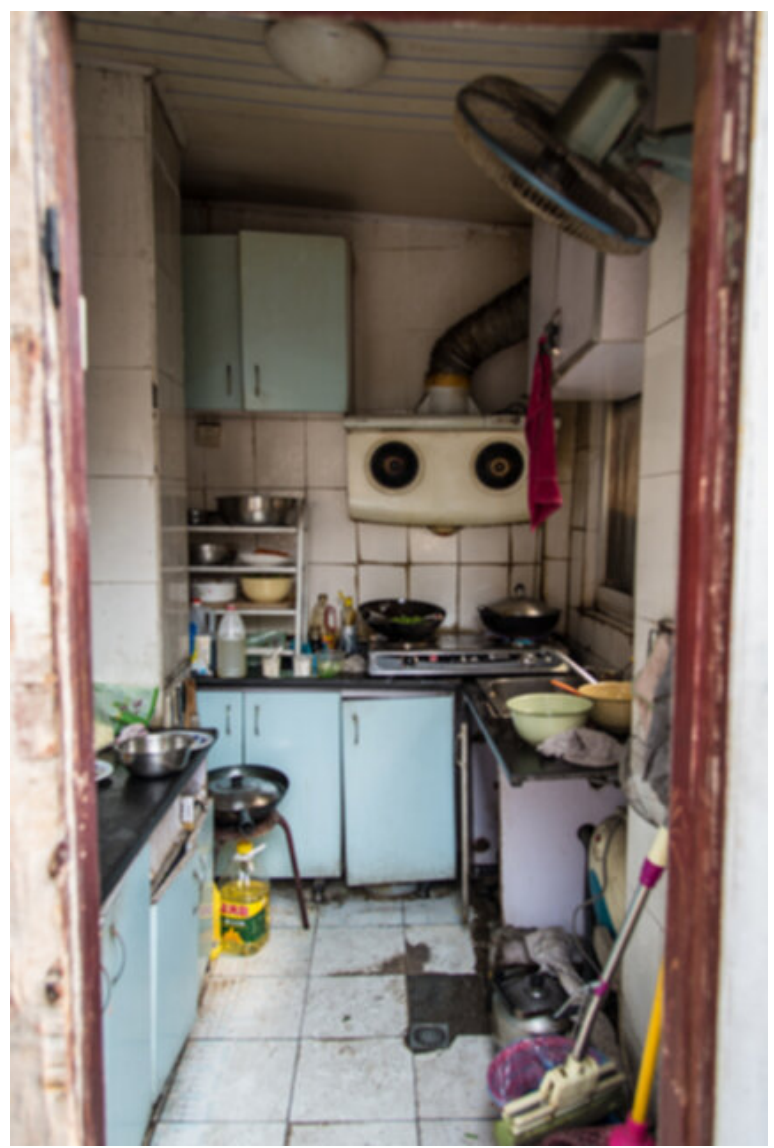

Question:are the cupboards open or closed ? MRR candidate set

1. closed

2. open

3. there is a cabinet slightly open

4. halfway open

5. ca n’t tell but looks closed

Top 10 from the remaining NDCG candidates 
1. i ca n't tell if there are doors

2. it is open

3. yes

4. i can t tell from this angle

5. yes,

6. no they are closed

7. no they are all closed

8. no

9. yes it is open

10. halfway it looks like

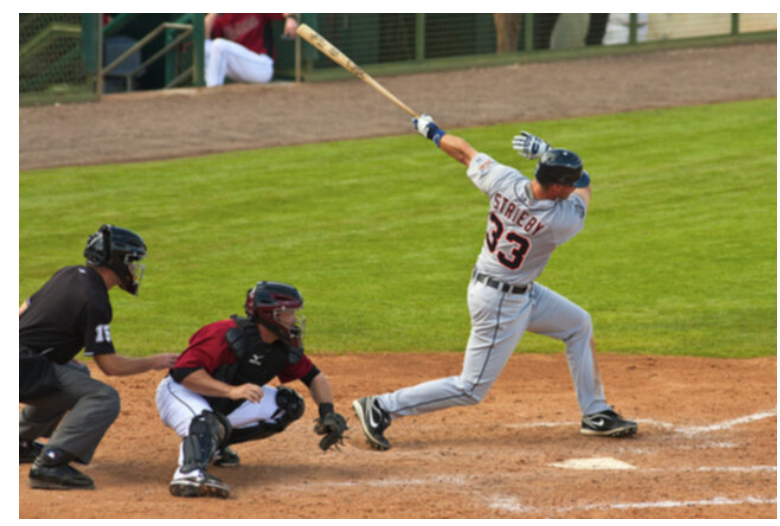

Question:is there only 1 baseball player?

\section{MRR candidate set}

1. 1 batting and a catcher

2. yes

3. yes there is

4. 1 that is visible yes

\section{Top 10 from the remaining NDCG candidates}

1. only 3 players can be seen from the angle

2. yes, he plays for the mets

3. no i see 2

4. there are 2

5. it appears to be

6. es

7. no

8. yes about 5

9. yes, it is
10. $\mathrm{i}$ think so

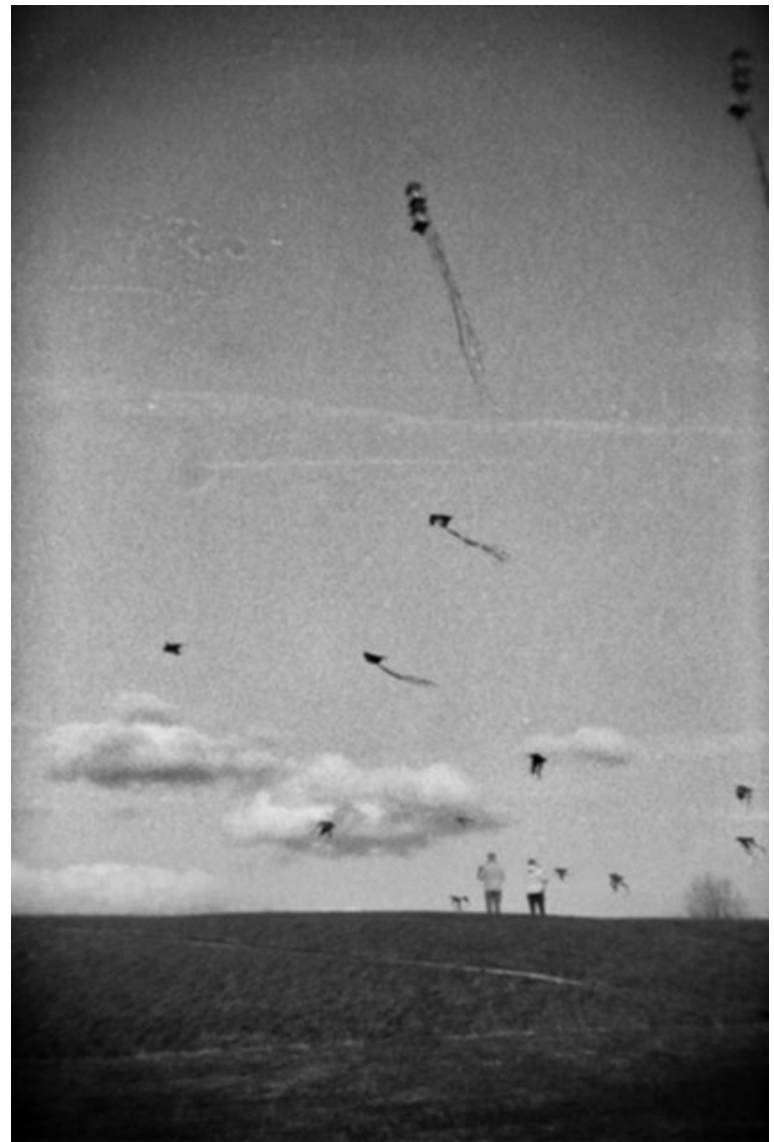

Question:how old do they seem to be?

\section{MRR candidate set}

1. they are pretty far away but seem to be an older couple

2. i can not tell

3. i ca n't tell

4. ca n't tell

\section{Top 10 from the remaining NDCG candidates}

1. middle aged

2. i ca n't tell his back is facing the camera

3. i would say 30 s

4. maybe mid thirties

5. not sure

6. $20-30$

7. pretty old, in their $60 \mathrm{~s}$

8. 20 's or 30 's

9. mid thirties 
10. maybe late 20 's to early 30 's

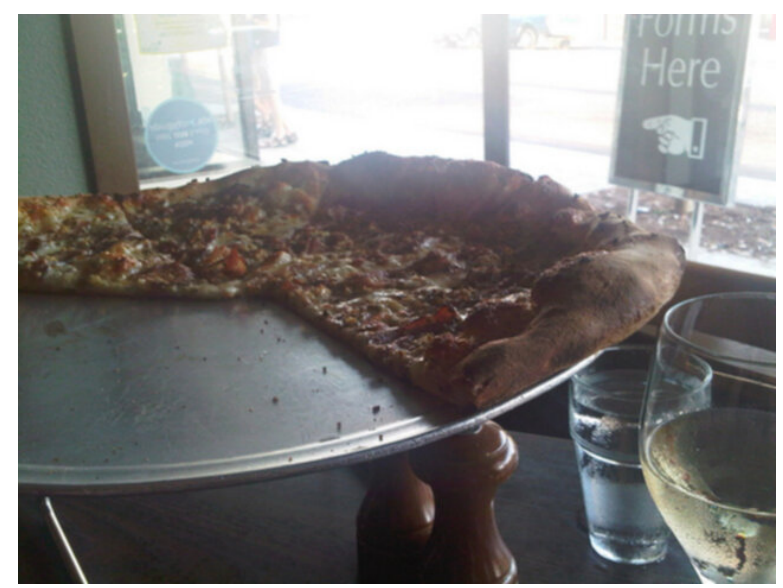

Question:are there any utensils shown?

MRR candidate set

1. no

2. yes

3. nope

4. no there are n't

5. yes, there are

\section{Top 10 from the remaining NDCG candidates}

1. not that i can see

2. yes there are some

3. not really

4. i do n't think so

5. 0

6. i think so

7. 0 at all

8. yes a glass bowl

9. lots

10. 1

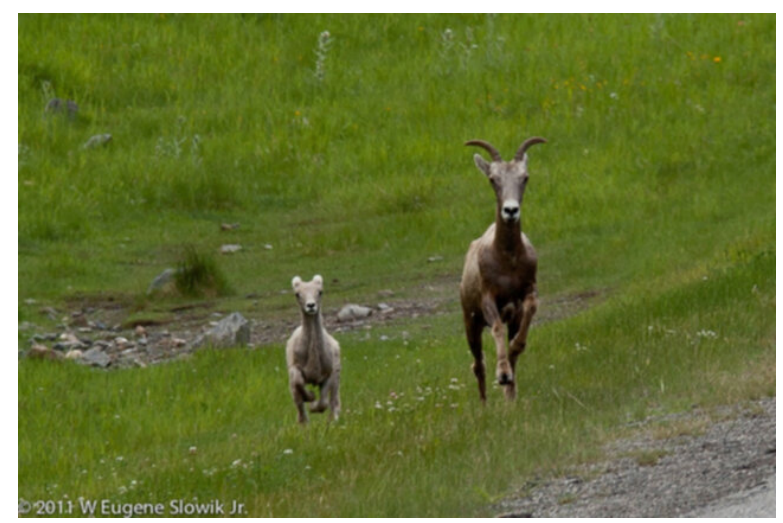

Question:is the photo in color?

\section{MRR candidate set}

1. yes

2. yes it is

3. yes, it is

4. yes, it 's a color image

5. yes, the image is in color

6. yes it 's in color

\section{Top 10 from the remaining NDCG candidates}

1. yes, the picture is in color

2. yes the picture is in color

3. yes in color

4. it is

5. it is in color

6. yes,

7. yep

8. definitely

9. i believe it is, yes

10. yes, it look professional



Question:approximately how many people are there?

\section{MRR candidate set}

1. 6

2. 2 in the foreground, about 6 in the back

3. 4

4. 5 

5. 5 people
6. i see 4 people

Top 10 from the remaining NDCG candidates

1. $\mathrm{i}$ see 7

2. 3

3. there are 4 total

4. 8

5. 3 that $\mathrm{i}$ can see

6. about 10

7. around 9

8. 4 or 5 , they are very tiny and hard to count

9. about 3

10. looks like 3

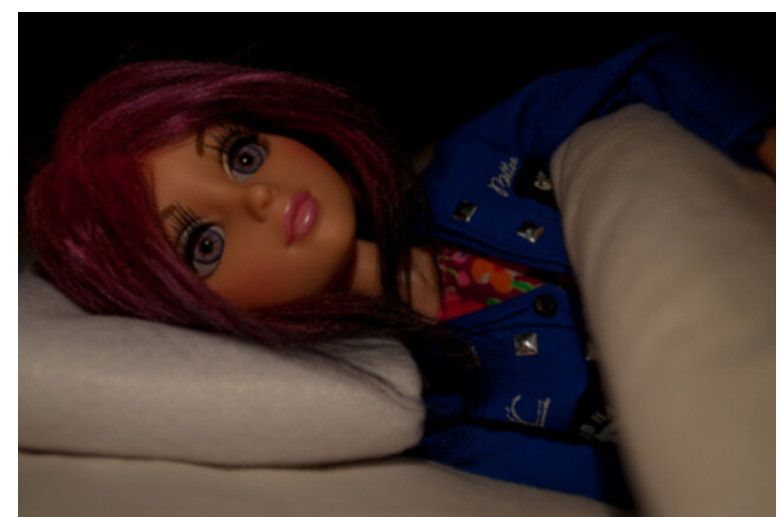

Question:is there any people?

\section{MRR candidate set}

1. no

2. just a photo of 1

3. nope

4. not that $\mathrm{i}$ can see

Top 10 from the remaining NDCG candidates

1. no there is n't

2. no people

3. not in the image

4. 0 that $\mathrm{i}$ can see

5. i do n't see any

6. no 1
7. no people in the shot

8. no people present

9. there are no people

10. 0



Question:is it clean?

MRR candidate set

1. yes

2. kind of, it 's under construction

3. no

4. yes, it is

\section{Top 10 from the remaining NDCG candidates}

1. appears to be

2. yes it is

3. it looks like it

4. somewhat

5. hard to tell

6. kind of

7. i think so

8. sort of 
9. not really

10. nope

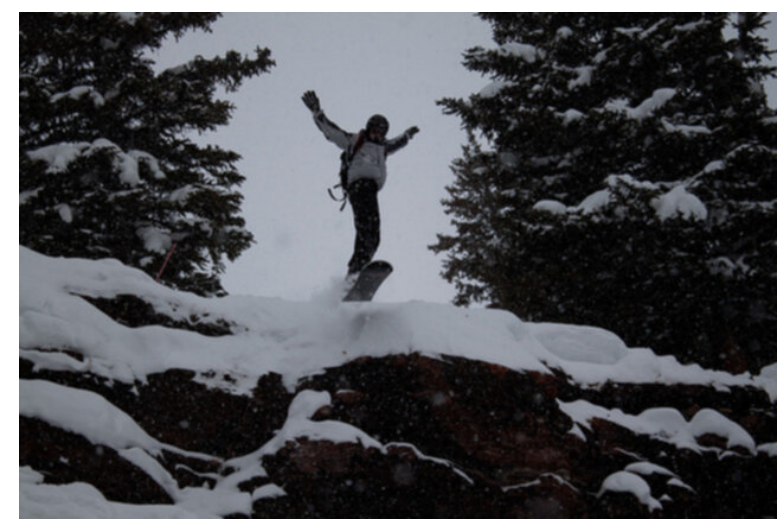

Question:is it a male?

MRR candidate set

1. yes

2. hard to tell

3. i can not tell

4. is like that

5. ca n't tell

6. i ca n't tell

\section{Top 10 from the remaining NDCG candidates}

1. not sure

2. i think so

3. possibly, i ca n't tell

4. no sure

5. looks like

6. it appears to be

7. could be

8. seems to be

9. yes, it is

10. yes it is

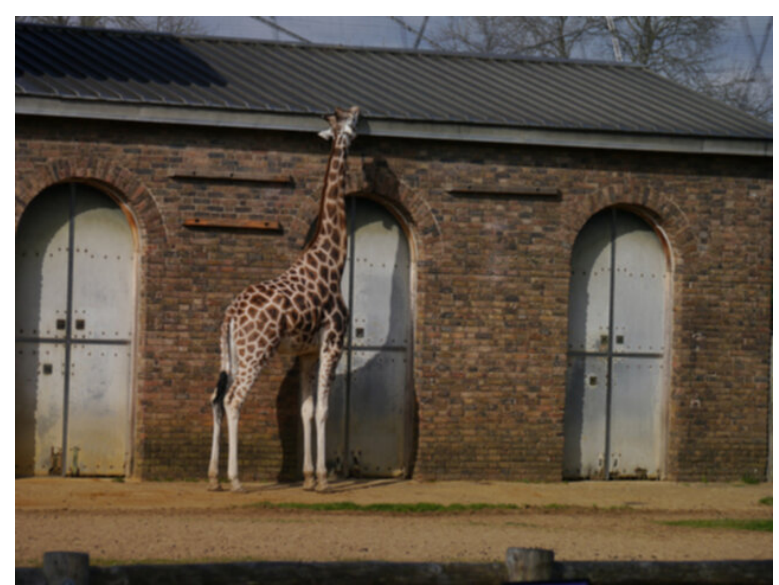

Question:is the weather UNK?

MRR candidate set

1. yes, it is

2. it looks a bit overcast

3. it looks clear

4. i can not tell

5. no

6. i ca n't tell

\section{Top 10 from the remaining NDCG candidates}

1. ca n't tell

2. yes, it is very clear

3. no it is not

4. ca n't tell from photo

5. it is sunny

6. i do n't think so

7. yes

8. no it's not

9. yes, from what i can see

10. yes, the sun is shining

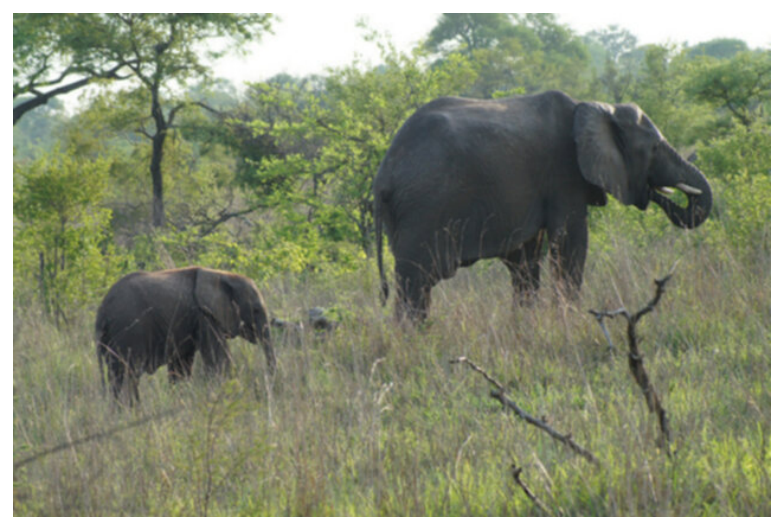


Question:would you say this is a mother and child ?

\section{MRR candidate set}

1. yes i would

2. yes

3. possibly

4. i think so

5. yes it is

6. not sure

Top 10 from the remaining NDCG candidates

1. maybe

2. i can not tell

3. yes, it is

4. i ca n't tell

5. ca n't really tell, but i would believe so

6. no

7. no can not tell

8. i do n't think so

9. ca n't tell

10. nope

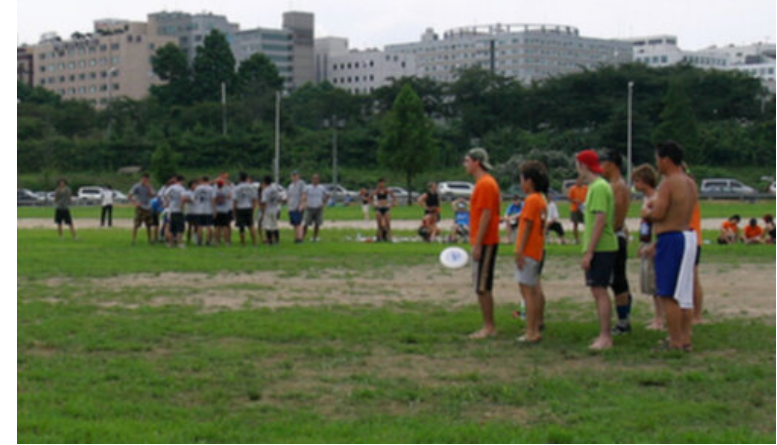

Question:is it day or night?

\section{MRR candidate set}

1. day

2. daytime

3. it is daytime

4. day time
5. it 's daytime

\section{Top 10 from the remaining NDCG candidates}

1. it looks to be day time

2. it is mid day

3. day time i think

4. it looks like it may be late afternoon

5. in the even or morning

6. probably afternoon

7. yes

8. yes it is

9. morning

10. yes, it is

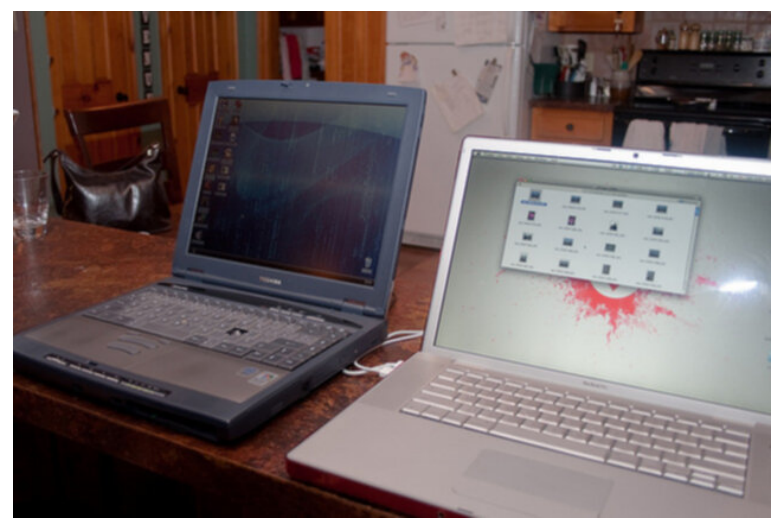

Question:what brand of laptop are they? MRR candidate set

1. toshiba, i ca n't tell on the other

2. i can not tell

3. i ca n't tell

4. ca n't tell

5. not sure

Top 10 from the remaining NDCG candidates

1. no sure

2. hard to tell

3. i do n't know

4. can 's really tell

5. you ca n't see it, but if it is truly a laptop you can only see the screen , 
6. not necessarily

7. silver

8. hard to tell, some kind of pine i think

9. all gray

10. grey

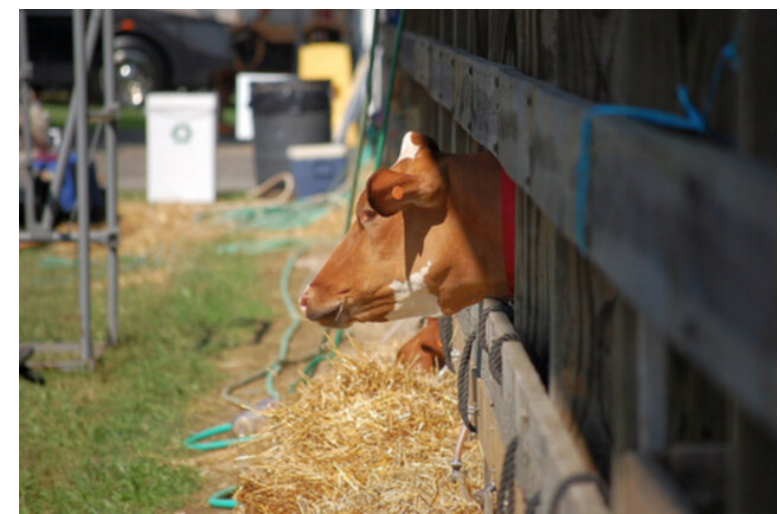

Question:can you see any other cows ? MRR candidate set

1. no

2. nope

3. no i can not

\section{Top 10 from the remaining NDCG candidates}

1. no, i ca n't

2. just the 1

3. only 1

4. i do not

5. no i do n't

6. not that i can see

7. 0 at all

8. no3

9. 0

10. i do n't think so

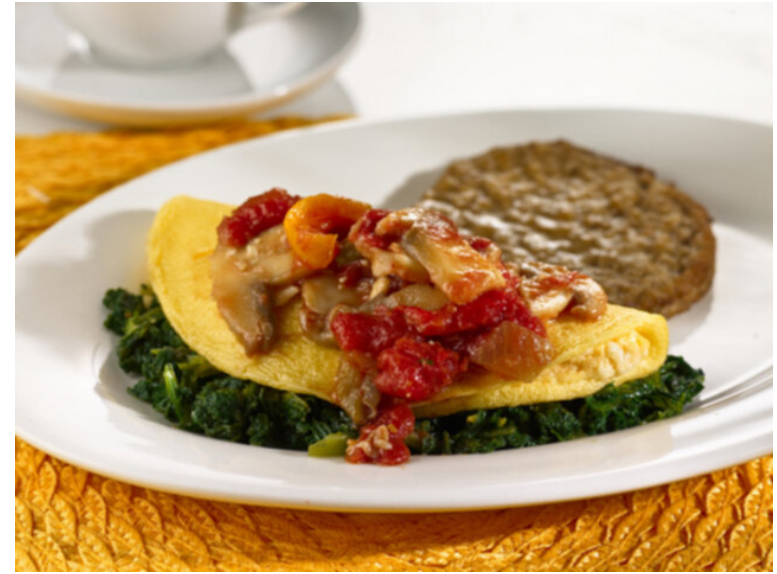

Question:is there a bun?

MRR candidate set

1. yes

2. yes, there is

3. yes there is

4. there is

Top 10 from the remaining NDCG candidates

1. no

2. yep

3. nope

4. yes, $\mathrm{i}$ think so

5. no there is n't

6. yes on the side

7. yes, it is

8. i think so

9. not that i can see

10. i do n't think so 